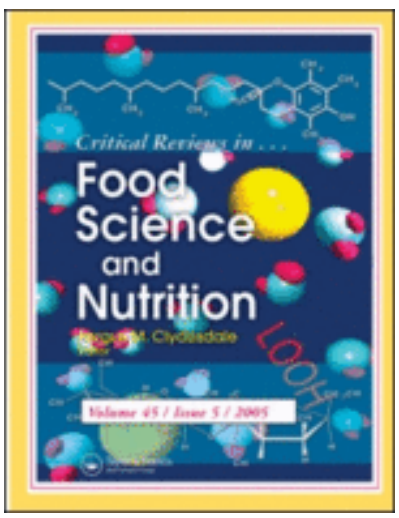

\title{
Plant-Based Foods As a Source of Lipotropes for Human Nutrition: a Survey of In Vivo Studies
}

\begin{tabular}{|r|l|}
\hline Journal: & Critical Reviews in Food Science and Nutrition \\
\hline Manuscript ID: & Draft \\
\hline Manuscript Type: & Review \\
\hline Date Submitted by the & \\
Author: & \\
\hline Complete List of Authors: & Fardet, Anthony; INRA, Human Nutrition \\
\hline Keywords: & Phytochemicals, lipotropes, hepatic steatosis, humans, rats \\
\hline
\end{tabular}

\section{S) ScholaroNE" \\ Manuscript Central}




\section{Plant-Based Foods As a Source of Lipotropes for Human Nutrition: a Survey of 2 In Vivo Studies}

3

4 ANTHONY FARDET, PhD, and JEAN-MICHEL CHARDIGNY, $\mathrm{PhD}^{\mathrm{a}}$

5

6

8

${ }^{a}$ INRA, UMR 1019 Nutrition Humaine, F-63122 Saint Genès Champanelle, France ; Clermont

Université, UFR Médecine, UMR 1019 Nutrition Humaine, F-63000, Clermont-Ferrand, France ;

CRNH Auvergne, F-63000 Clermont-Ferrand, France.

Address correspondence to Anthony Fardet, INRA, UMR 1019 Nutrition Humaine, F-63122 Saint 19 Genès Champanelle, France ; Clermont Université, UFR Médecine, UMR 1019 Nutrition Humaine, 20 F-63000, Clermont-Ferrand, France ; CRNH Auvergne, F-63000 Clermont-Ferrand, France, Tel. 21 +33(0)4 736247 04, Fax. +33(0)4 736247 55. E-mail: anthony.fardet@clermont.inra.fr 


\section{Abstract}

2 Increased consumption of plant products is associated with lower chronic disease prevalence. This

3 is attributed to the great diversity of their phytochemicals and to their numerous positive

4 physiological effects. The most investigated have been their antioxidant, anti-carcinogenic,

5 hypolipidemic and hypoglycemic properties. Yet, some compounds have been very early shown to

6 be lipotropic in animals. This property is defined as the capacity of a compound to hasten the

7 removal of fat from liver and/or to reduce hepatic lipid synthesis through several mechanisms,

8 mainly involving increased phospholipid synthesis via the transmethylation pathway for

9 triglyceride-rich lipoprotein exportation from liver, increased fatty acid $\beta$-oxidation and/or down-

10 and up-regulation of genes involved in respectively lipogenic and fatty acid oxidation enzyme 11 synthesis. Main plant lipotropes are choline, betaine, myo-inositol, methionine and carnitine. 12 Magnesium, niacin, pantothenate and folates also indirectly support the overall lipotropic effect. 13 The exhaustive reviewing of animal studies investigating the effect of phytochemicals on hepatic 14 lipid metabolism suggest that some unsaturated fatty acids, acetic acid, melatonin, phytic acid, some 15 fiber, oligofructose, flavonoids, lignans, stilbenes, curcumin and saponins may be also considered 16 as having lipotropic effects. However, this will have to be confirmed in humans for which 17 intervention studies are practically non-existent.

19 Keywords: Phytochemicals, lipotrope, hepatic steatosis, humans, rats 


\section{PLANT-BASED FOOD CONSUMPTION, CHRONIC DISEASE RISK AND}

\section{PHYTOCHEMICALS}

$3 \quad$ Epidemiological and observational studies

4 Increased consumption of plant-based foods (PBF), mainly whole-grain cereals, legumes,

5 vegetables and fruits, is generally associated with a lower prevalence of all-cause mortality and of 6 the major chronic diseases that are cardiovascular diseases (CVD), obesity, diabetes and cancers. 7 However, more specifically, the effects seem to vary according to the botanical origin of the PBF 8 with more or less conclusive results from prospective studies. Thus, while whole-grain cereals have 9 been convincingly shown to be protective against all main chronic diseases or disorders (Chan et 10 al., 2007; Chatenoud et al., 1998; De Munter et al., 2007; Flight and Clifton, 2006; Jacobs et al., 11 2007; Jacobs et al., 1998; Koh-Banerjee et al., 2004; Koh-Banerjee and Rimm, 2003; Larsson et al., 12 2005; Mellen et al., 2008; Murtaugh et al., 2007; Sahyoun et al., 2006; Schatzkin et al., 2008; Van 13 De Vijver et al., 2009; Venn and Mann, 2004; Williams et al., 2008), the effects of legumes, fruits 14 and/or vegetables are less obvious with both either no or positive effects reported that depends on 15 the variety used, the population studied, the targeted disease or the age of the subjects. For example, 16 the protective role of PBF and plant-based diets against childhood obesity has been recently 17 reviewed: it clearly appears that, except for ready-to-eat cereals, there is a lack of evidence to 18 conclude for an association between PBF and childhood obesity in relation with fruit and vegetable, 19 grain other than cereal, and legume intake (Newby, 2009).

To summarize, the most conclusive associations are observed with whole-grain cereals for all diseases, with legumes on mortality risk (all-cause, CVD or cancers) (Nagura et al., 2009; 22 Noethlings et al., 2008), with fruits on CVD (Hung et al., 2004; Nagura et al., 2009) and weight 23 gain/obesity (Buijsse et al., 2009; He et al., 2004), with vegetables on CVD (Hung et al., 2004; 24 Nagura et al., 2009), weight gain/obesity (Buijsse et al., 2009; He et al., 2004) and type 2 diabetes 25 (Bazzano et al., 2008; Villegas et al., 2008), and with both fruits and vegetables on all-cause 26 mortality (Rissanen et al., 2003; Steffen et al., 2003) and cancers (Pavia et al., 2006; Van 
1 Duijnhoven et al., 2009) (Table 1). Moreover, some authors have observed a significant association

2 between diseases risk and mortality with specific vegetable or fruit sub-family consumption such as 3 cruciferous, Alliaceae, green leafy and yellow-orange vegetables, root vegetables, citrus or fruit-

4 berry. This is underlined for cancer (Kolonel et al., 2000; Wu et al., 2009), diabetes (Bazzano et al., 5 2008), cerebrovascular disease (Mizrahi et al., 2009) and all-cause mortality (Nagura et al., 2009; 6 Noethlings et al., 2008) risks. More specifically, the inverse association between green tea 7 consumption and psychological distress in a Japanese cohort has been recently reported (Hozawa et 8 al., 2009). Studies reporting increased prevalence of chronic diseases with increased consumption 9 of PBF are practically non-existent except one Chinese study that reported increased prevalence of 10 obesity among high consumers of vegetables but the culinary habits involved the cooking of 11 vegetables with important amount of oil for stir-frying (Shi et al., 2008). Despite some 12 contradictory reported results, or at least the absence of significant effect, PBF consumption does 13 not appear negative for health on a long term, provided they are not systematically accompanied 14 with sausages or other energy-dense seasonings and snack foods. It is therefore certain that 15 increasing its PBF consumption is not unhealthy, if not always reflected in a significant health 16 benefit.

\section{A whole set of phytochemicals with numerous physiological effects}

21 The overall potential positive effect of PBF on chronic diseases would be associated with the 22 presence, especially in unrefined and/or minimally-processed PBF, of a great variety of 23 phytochemicals (vitamins, minerals, trace elements, carotenoids, polyphenols, phytosterols,...) 24 together with the fibre fraction of PBF which would act synergistically to favour various positive 25 physiological effects (Slavin, 2003). The mechanisms may involve (1) the chelation, reduction 26 and/or trapping of free oxidative radicals (i.e. the antioxidant capacity) (Fang et al., 2002; Pellegrini 
1 et al., 2003; Wu et al., 2004a), (2) the stimulation/modulation of the immune function (Barr et al.,

2 1998; Mantovani et al., 2008), (3) the regulation of glucose homeostasis (e.g. magnesium stimulates

3 the glucose uptake by insulin) (Venn and Mann, 2004), (4) the lowering of circulating or liver

4 damaging lipid fractions (e.g. LDL-cholesterol) (Lee et al., 2005; Okazaki and Katayama, 2008),

5 (5) the reduction of hyperhomocysteinemia recognized as a risk factor for CVD (Graham et al.,

6 1997; Samman et al., 2002) and for carcinogenesis (Wu and $\mathrm{Wu}, 2002)$, (6) the anti-carcinogenicity

7 or the capacity to induce apoptosis (Azzi and Stocker, 2000; Rubis et al., 2008; Shamsuddin, 2002),

8 and/or (7) the anti-aggregability (Shechter et al., 1999) and anti-inflammatory (Liu et al., 2004;

9 Rahman et al., 2006) properties of polyphenols and other micronutrients richly contained in the bran

10 and germ fractions of cereals but also in whole-grain legumes, fruits and vegetables (Azzi and

11 Stocker, 2000; Eastwood, 1999; Fardet, 2009; Lotito and Frei, 2006; Prior, 2003; Thompson et al.,

12 2005). As demonstrated more recently, the up- or down-regulation of cell redox status via

13 signalling-related mechanisms, of glutathione synthesis and/or of genes involved in the

14 development of chronic diseases (Azzi and Stocker, 2000; Moskaug et al., 2005; Rahman et al.,

15 2006), notably through the action of polyphenols and/or their metabolites (Horev-Azaria et al.,

16 2009; Hsu and Yen, 2008), would also be involved. Today, one agrees to advance that several

17 phytochemicals are involved in each of these physiological mechanisms through a synergetic effect.

18 For example, the antioxidant capacity of fruits, vegetables and whole-grain cereals is attributed to

19 very different compounds such as polyphenols, vitamins E and C, selenium, phytic acid... In other

20 words, one compound may exert several protective functions and several phytochemicals may act

21 synergistically to counteract the development of one damaging physiological process as we have

22 recently reviewed it for the protective mechanisms associated with whole-grain cereal consumption

23 (Fardet, 2009).

It is therefore more and more admitted that a small amount of a cocktail of

25 phytomicronutrients would be more beneficial than only one or two phytomicronutrients at high

26 doses as recently demonstrated in healthy women consuming either 18 botanical families of 
1 vegetables and fruits with a modest antioxidant effect or 5 botanical families with a high reported in 2 vivo antioxidant activity (Thompson et al., 2006). Moreover, similarly to the increased oxidative 3 stress that has been shown to be involved in most of the previously cited chronic diseases (Bartsch 4 and Nair, 2006,Castelao and Gago-Dominguez, 2008; Keaney et al., 2002; Maiese et al., 2007), 5 other impaired physiological mechanisms may be common to different metabolic disorders, such as 6 increased inflammation, immuno- or glucose homeostasis dysregulation, and/or hyperlipidemia in 7 plasma or liver. However, the number of different phytochemicals contained in PBF is so high that 8 the elucidation of all the mechanisms involved will be a long lasting and difficult task.

\section{PLANT-BASED FOODS AS DIETARY SOURCES OF LIPOTROPES} 12

Betaine, choline, myo-inositol and methionine in plants

Although discovered a very long time ago in plants, some of them have been rather neglected when compared to studies related to health potential of minerals, trace elements, vitamins and more recently polyphenols. These compounds are choline, betaine and myo-inositol, this latter being a natural isomer of glucose that belongs to the cyclitol family (Figure 1). They have been mostly studied as isolated compounds and often at non-nutritional doses. In plants, betaine has choline as precursor. Betaine and choline are water soluble cytoplasmic osmolytes and thermoprotectants that play a regulatory role in situation of stress for the plant, notably in water-depressed (drought), saline and temperature-stressed environments (Caldas et al., 1999; Hanson and Hitz, 1982; Hanson and Wyse, 1982; Hitz et al., 1982; Ladyman et al., 1980; Nolte et al., 1997; Summers and Weretilnyk, 1993). whole-grain pseudocereals such as amaranth and quinoa) and Gramineae- (i.e. whole-grain cereals) 
1 derived plants are well recognized for their high betaine content, as a result of an adaptation to

2 environmental stress (Craig, 2004; Hanson and Hitz, 1982; Hanson et al., 1985; Hanson and Wyse, 3 1982; Hitz et al., 1982; Yokoishi and Tanimoto, 1994) 4 for betaine and choline contents confirmed these observations (USDA, 2008). Except fruits, PBF 5 are generally a good source of choline, particularly whole-grain cereals, wheat bran and germ, leafy 6 vegetables and soybean (USDA, 2008).

Otherwise, choline and myo-inositol are important constituents of cell membranes as 8 precursors of phosphatidylcholine and phosphatidylinositol. In many plants, myo-inositol is also the 9 basic constituent of myo-inositol phosphate or phytate (IP6) that plays a role as phosphorus and 10 myo-inositol stores used for future seed development, but also as regulator of inorganic phosphate 11 levels (Lott et al., 2000). Among PBF, whole-grain cereals, legumes, nuts and seeds contain the 12 highest levels of phytate (Harland and Oberleas, 1987; Lott et al., 2000; Reddy et al., 1982). On the 13 other hand, myo-inositol may be also present as free or conjugated (e.g. glycosylated myo-inositol 14 or galactinol) soluble compound, as in citrus fruits where free myo-inositol content may reach up to 15 nearly $7 \%$ of total sugars in lemon (Masuda et al., 2003) and concentrations up to $153 \mathrm{mg} / 100 \mathrm{~mL}$ 16 in fresh juice from kiwifruit (Sanz et al., 2004). Although literature data are scarce, the richest 17 sources of free or conjugated myo-inositol appear to be legumes (Schweizer et al., 1978; Sosulski et 18 al., 1982), wheat germ (Horbowicz and Obendorf, 1994), pseudo-cereals (Becker et al., 1981; 19 Koziol, 1992) and fruits (Clements and Darnell, 1980), especially citrus (Masuda et al., 2003; Sanz 20 et al., 2004).

Concerning methionine, it is an essential aminoacid especially found in high amounts in 22 cereals, legumes, nuts and seeds (USDA, 2005b, 2005c, 2005d).

The lipotropic effect of betaine, choline, myo-inositol and methionine

25 In humans, betaine (Craig, 2004), choline (Zeisel and Costa, 2009) and myo-inositol (Clements and 26 Reynertson, 1977; Fux et al., 1996; Sundkvist et al., 2000) have been shown to exert multi-factorial 
1 physiological effects. Being essential nutrients for human organism, they were cited as vitamins

2 (vitamin I for myo-inositol, vitamin B10 for betaine and vitamin $\mathrm{J}$ for choline) for a quite long time 3 in some scientific articles, especially myo/meso-inositol and choline (Calhoun et al., 1958; Calhoun 4 et al., 1960; Ournac, 1970; Scriban, 1970; Seifert, 1972). Yet, the vitaminic status of choline has 5 been very early debated and it was concluded in 1944 that "it would appear to be more satisfactory 6 to leave choline unclassified" due to the lack of scientific evidences (Mchenry and Patterson, 1944).

7 These compounds, notably betaine, are yet still today presented as vitamins on some web sites, but 8 not in scientific literature. Betaine and choline are first well-known as methyl donors able to 9 stabilize the plasma homocysteine level (Craig, 2004; Olthof and Verhoef, 2005; Sanders and 10 Zeisel, 2007), hyperhomocysteinemy being a risk factor for CVD (Eikelboom et al., 1999; Graham 11 et al., 1997).

12 Betaine, choline and myo-inositol have been first very early shown to have the particularity 13 to exert lipotropic effect within animal liver (Best, 1934; Best and Huntsman, 1932; Best and 14 Huntsman, 1935; Gavin and Mchenry, 1941a; Owens, 1942; Perrault and Dormard, 1966; Thuillier, 15 1956) (Supplemental Table 1). Although betaine and choline were discovered during the $19^{\text {th }}$ 16 century in respectively beet juice and ox bile (1862) - chole is bile in greek (Li and Vance, 2008), 17 the term "lipotropic" was first used only in 1935 by Best et al. who showed that choline is able to 18 prevent and cure fatty livers in rats and that increased liver fat infiltration and accumulation was 19 primarily due to deficiency in some essentials factors whose the principal role is to assure lipid 20 transport and turnover (Best, 1935). Today, one defines lipotropes as compounds that act on lipid 21 metabolism by preventing fat accumulation within the liver through hastening fat removal or by 22 preventing excessive fat deposits (e.g. accumulation of cholesterol).

The prevalence of NAFL and NASH in the general population of the United States is estimated at $20 \%$ and $3 \%$ 23 respectively and can be as high as $95 \%$ in high-risk subgroups with abnormal liver enzymes,type 2 diabetes mellitus, or morbid obesity \{Falck-Ytter, 2001 \#20830\}. 57

26 generally observed in situations of alcohol excess (Lieber, 1997), obesity, overweight and diabetes 
1 (James and Day, 1998; Patrick, 2002; Sharabi and Eldad, 2000; Shimada et al., 2002; Silverman et

2 al., 1990; Silverman et al., 1989). A fatty liver is vulnerable and steatosis may lead to steatohepatitis

3 (hepatocellular inflammation), fibrosis or cirrhosis, but not systematically (Adams et al., 2005;

4 Angulo and Lindor, 2001; Day and James, 1998a; James and Day, 1998). Moreover, patients with

5 hepatic steatosis present an increased risk of developing CVD (Mannarino et al., 2009). In addition,

6 fatty liver is often associated with a cluster of several impaired physiological mechanisms including

7 insulin resistance (Gastaldelli et al., 2009; Mamone et al., 2009; Marchesini et al., 1999; Patrick,

8 2002; Seppala-Lindroos et al., 2002; Valtuena et al., 2006), increased oxidative stress (Day and

9 James, 1998a; Day and James, 1998b; Kwon et al., 2009a; Reid, 2001), hyperlipidemia (Brouwers

10 et al., 2005; James and Day, 1998; Sharabi and Eldad, 2000; Shimada et al., 2002; Vuppalanchi and

11 Chalasani, 2009), metabolic syndrome symptoms (Cortez-Pinto et al., 1999; Mannarino et al., 2009;

12 Patrick, 2002; York et al., 2009伭, endothelial dysfunction and arterial stiffness (Mannarino et al.,

13 2009), and hepatocarcinogenesis (Shimada et al., 2002; Yatsuji et al., 2006). A minimum of 5-10\%

14 hepatic steatosis or fat accumulation by weight is generally considered to diagnose non-alcoholic

15 fatty liver (NAFL) (Neuschwander-Tetri and Caldwell, 2003). And steatosis is considered mild

16 (grade 1), moderate (grade 2) or severe (grade 3) when respectively $<33 \%, 33-66 \%$ or $>66 \%$ of 17 hepatocytes are affected (Angulo, 2002; Brunt et al., 1999).

18 The development of fatty liver mainly results from the following metabolic dysfunctions: 1) 19 enhancement of fatty acid (FA) synthesis, 2) increased mobilization of FA from adipose tissues, 3) 20 inhibition or impairment of mitochondrial FA $\beta$-oxidation (Fromenty and Pessayre, 1995), 4) 21 increased transformation of FA into triglycerides (TG) by esterification, and 5) decreased release of 22 TG from liver (that notably naturally occurs via VLDL in a healthy liver) that can result from 23 decreased ApoB or microsomal TG transfert protein (MTP) syntheses (Jamil et al., 1998). All of 24 these mechanisms are particularly involved in situation of insulin resistance or hyperinsulinaemia 25 (Adams et al., 2005). 
Otherwise, in humans with non-alcoholic fatty liver diseases (NAFLD), increased long-

2 chain poly-unsaturated FA (PUFA) n-6/n-3 ratio was also observed and authors concluded that such

3 "condition may favour lipid synthesis over oxidation and secretion" (Araya et al., 2004). Indeed,

4 imbalanced diets generally lead to increased PUFA n-6/n-3 ratio that reduces PPAR $\alpha$ activation and

5 increases SREBP-1 (sterol regulatory element binding protein) expression, both mechanisms

6 leading to respectively decreased peroxisomal/mitochondrial $\beta$-oxidation and increased ApoB-100

7 degradation (that means a reduction of TG exportation from liver via VLDL), and to enhanced FA

8 and TG synthesis (Araya et al., 2004). The depletion in long-chain PUFA of the n-3 and n-6 series

9 might notably result from both their increased peroxidation in situation of increased oxidative stress

10 and inadequate intake (Araya et al., 2004). In obese patients, higher hepatic mRNA levels of

11 SREBP-1c (+33\%) and fatty acid synthase (FAS) (+70\%), higher SREBP-1c/PPAR $\alpha$ ratio $(+62 \%)$

12 with a concomitant reduced level of hepatic long-chain PUFA n-3 (-53\%) and insulino-resistance,

13 as compared to non-obese subjects, were reported and proposed as conditions that would favour 14 lipogenesis to the detriment of FA oxidation (Pettinelli et al., 2009).

In the case of NAFL associated with insulin resistance, the increased hepatic free fatty acid

16 (FFA) synthesis from glucose not uptook by peripheral adipocytes is also involved; while, in the 17 case of obesity, increased amounts of FFA simply enter the liver (Patrick, 2002). In presence of 18 excess FA, the mitochondrial $\beta$-oxidation pathway thus becomes an insufficient way of degrading 19 excess fat that accumulates in TG stored within cytoplasm. Excess TG may be also secreted in 20 plasma via VLDL leading to hypertriglyceridemia (Pagano et al., 2002). In the end, the increased 21 level of lipid peroxidation in hepatosteatosis generates more free radicals that may lead to 22 mitochondrial DNA damages and inhibit further lipid $\beta$-oxydation (Patrick, 2002). Thus, in a rat 23 nutritional model of hepatic steatosis with inflammation (following a 4-week methionine-choline24 deficient diet) - that is morphologically similar to non-alcoholic steatohepatitis in humans 25 significant increased in hepatic microsomal CYP2E1 (cytochrome P450 2E1) content was reported, 
1 this effect generating more reactive oxygen species that may damage liver cells (Weltman et al., 2 1996).

In the case of high-cholesterol diet, it has been shown in rats that cholesterol lead to specific depressed activities of mitochondrial phosphatidylcholine and phosphatidylethanolamine 24 hours after i.p. injection of $\left[1-{ }^{-14} \mathrm{C}\right]$ acetate (respectively around $-84 \%$ and $-64 \%$ ) (Morin, 1967), both compounds being essential for PL synthesis, then LDL exportation from liver. Authors suggested that cholesterol may have selectively decreased rate of synthesis and turnover from acetate for these compounds to the benefits of other phospholipids (PL) containing linoleic, eicosatrienoic acid, and arachidonic acids (Morin, 1967)

In the case of alcohol-induced fatty liver, excess ethanol consumption lead to increased hepatic lipogenesis from excess acetyl-CoA generated by ethanol metabolism. More specifically, the down-regulation of the $\operatorname{PPAR} \alpha$ (peroxisome proliferator-activated receptor) - as shown in vitro on hepatocytes in presence of ethanol (Galli et al., 2001) - appear to be specifically involved; and mitochondrial DNA deletions have been observed in patients with microvesicular alcoholic fatty liver (Fromenty et al., 1995). In addition, increased oxidative stress is also particularly involved: thus, by measuring ethane exhalation in high-alcohol consumers, hepatic fat deposits were suggested to be the factor leading to increased lipid peroxidation via increased production of oxygen radicals following mitochondrial changes in the respiratory chain (Lettéron et al., 1993). Other mechanisms have been unravelled in rats and minipigs chronically fed alcohol. They involve: alteration of hepatic methylation via inhibition of methionione synthase that allows methionine synthesis from homocysteine (Barak et al., 1997; Barak et al., 1987), decreased levels of S-adenosyl methionine (i.e. abnormal/altered methionine metabolism) that leads to depressed phosphatidylcholine synthesis (Figure 2A) (Esfandiari et al., 2007), increased SREBP-1C expression that has acetyl-CoA carboxylase (ACC), FAS and glycerol-3-phosphate acyltransferase as target genes (Esfandiari et al., 2007), decreased methionine synthase activity (MS, Figure 2A) (Halsted et al., 2002), and a suppressive effect on the phosphatidylethanolamine- $\mathrm{N}$ - 
1 methyltransferase pathway (PEMT, Figure 2A) (Zivkovic et al., 2009). However, upon prolonged

2 period of alcohol consumption, concomitant increased hepatic betaine homocysteine $s$ -

3 methyltransferase (BHMT) activity and decreased betaine levels were also observed, resulting from

4 an adaptation to methionine synthetase deficiency in order to yield sufficient amount of methionine

5 for S-adenosylmethionine synthesis (Figure 2A) (Barak et al., 1987). Both significant decreases in

6 methionine synthase and increase in BHMT have been also observed in micropigs chronically fed

7 alcohol upon 14 weeks, but, in this case, only when ethanol feeding was accompanied by folate 8 deficiency (Halsted et al., 2002).

$10 \quad$ Fatty liver or hepatic steatosis models

11 In animals - mainly rats and mice, fatty liver is generally provoked by using lipotrope-deficient 12 diets (Lombardi et al., 1968; Olson et al., 1958a), high-fat diet $(\approx 20-40 \%)$ (Borgschulte et al., 13 2008; Olson et al., 1958a; Ryu and Cha, 2003; Singal and Eckstein, 1939), high-cholesterol diet 14 (Felmlee et al., 2009), high-fructose/glucose/sucrose diet $(\approx 60 \%)$ (Hammond et al., 2003; Olson et 15 al., 1958a; Rosenfeld, 1973; Ryu and Cha, 2003; Sanchez-Lozada et al., 2010), low-PUFA diet 16 (Goheen et al., 1983; Keim and Mares-Perlman, 1984), orotic acid-supplemented diet (fatty liver 17 resulting from ApoB synthesis impairment) (Fukuwatari et al., 2002; Nagiel-Ostaszewski and Lau18 Cam, 1990; Vaishwanar et al., 1972) or ethanol-rich diet (Balkan et al., 2004; Barak et al., 1997; 19 Song et al., 2008). Fatty liver may be also provoked by single ethanol (Baker et al., 1973), carbone 20 tetrachloride $\left(\mathrm{CCl}_{4}\right)$ (Vaishwanar et al., 1972) or DDT (1,1,1-trichloro-2,2-bis (p-chlorophenyl) 21 ethane) (Okazaki et al., 2006) injections, via depleting hepatic carnitine levels by using chemicals 22 such as mildronate or THP (trimethylhydraziniumpropionate) (Degrace et al., 2007; Spaniol et al., 23 2003) or via hypercaloric and fat-free parenteral nutrition (Keim and Mares-Perlman, 1984). The 24 use of specific mice strains that mimic choline-deficient diet has also been reported (Dumas et al., 25 2006). There are still other animal models of steatosis, notably in relation with naturally occuring mutations in rats (e.g. obese fa/fa Zucker rats) and mice ( $\mathrm{db} / \mathrm{db}$ mice - diabetic dyslipidemia - or 
1 ob/ob mice - leptin-deficient), genetically modified mice or rats and mice treated with 2 environmental inhibitors of hepatic FA oxidation (e.g. glucocorticoids, estrogen antagonists, 3 tamoxifen, valproic acid or etomoxir - a CPT-1 inhibitor) (Angulo, 2002; Koteish and Diehl, 2001).

4 Conversely, KO mice for specific enzymes involved in lipogenesis may be used to limit the 5 development of fatty liver, e.g. mitochondrial glycerol-3-phosphate acyltransferase (mtGPAT) -/6 mice, mtGPAT catalysing the rate-limiting step in TG synthesis (Hammond et al., 2003).

7 In humans, as presented previously, hepatic steatosis is observed in situations of overweight, 8 obesity, diabetes, hyperlipidemia or alcohol excess. Otherwise, humans in situation of total 9 parenteral nutrition may exhibit choline deficiencies with a resulting hepatic steatosis (Buchman et 10 al., 2001; Buchman et al., 1995), but the high content in dextrose and glucose of parenteral 11 solutions might be also involved (Liang et al., 1999).

12 In the end, protein-calorie malnutrition, rapid weight loss or chronic starvation/food 13 deprivation may also lead to NAFLD in both humans (Adams et al., 2005; Angulo, 2002; Doherty 14 et al., 1992; Neuschwander-Tetri and Caldwell, 2003) and animals (Ginneken et al., 2007; 15 Nieminen et al., 2009; Yasuhara et al., 1991). Possible involved mechanisms may be in relation 16 with lipotrope depletion, and also n-3 PUFA depletion. Indeed, n-3 PUFA contribute to the 17 regulation of lipid metabolism, notably by inhibiting transcription of lipogenic genes and inducing 18 gene in relation with FA $\beta$-oxidation. In addition, starving lead specific hormonal profiles that can 19 promote TG hydrolysis into adipose tissues, FA products being thereafter taken up by the liver 20 where they may be newly synthesized into TG (Kersten et al., 1999).

22 Betaine, choline, myo-inositol, methionine and in vivo lipotrope-related studies

23 The lipotropic efficiency of betaine, choline and myo-inositol towards fatty liver has thus been 24 demonstrated since a long time by using lipotrope-deficient, high-fat/high-sucrose or ethanol25 enriched diets in rats as exhaustively reviewed in Supplemental Table 1 (Barak et al., 1997; Barak 26 et al., 1996a; Barak et al., 1996b; Best et al., 1950; Carroll and Williams, 1982; Chahl and Kratzing, 
1 1966a; Gavin and Mchenry, 1940; Halliday, 1938; Hayashi et al., 1974a). The efficiency was

2 notably determined through dose-response curves, choline being 3-fold the potency of betaine and 3 methionine and betaine being more efficient than myo-inositol (Best et al., 1950; Young et al., 4 1965). Microscopical observations confirmed the lower lipotropic potential of betaine compared to 5 choline (Ball, 1964). However, Andersen and Holub showed that, on a same molar basis of 5.4 $6 \mathrm{mmol} / \mathrm{kg}$ of diet, choline and myo-inositol had the same lipotropic effect towards hepatic TG

7 accumulation in rats fed a basal diet not supplemented with choline or myo-inositol suggesting that 8 previously reported efficiency ratios would differ according to the experimental scheme (Andersen 9 and Holub, 1980).

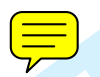

In humans, published results were scarcer. The first results reported in a scientific journal, to 11 our knowledge, were those of Broun and Muether in 1942: authors apparently based on the results 12 of Griffith and Mulford - obtained in rats and released one year before (Griffith and Mulford, 13 1941b) - to test choline chloride for more than 2 years in humans ( $1 \mathrm{~g}$ daily) with hepatic cirrhosis 14 (Broun and Meuther, 1942). They notably observed decreases in blood bilirubin and cholesterol, 15 elimination of ascites - i.e. accumulation of fluid into peritoneal cavity that may be TG-rich - and 16 decreased liver size (Broun and Meuther, 1942). Three years latter, Barclay and Cooke reported the 17 case of a 27 years-old man who had developed severe liver dysfunction (and renal failure) after 18 receiving large doses of barbiturates for anxiety state; and who was treated both orally (2-5 g for 19 one day) and intravenously (6-8 g) with high doses of choline chloride, then methionine (6 g) and 20 choline chloride during more than one month: recovery of the patient was noted despite important 21 side-effects related to the choline treatment (i.e. fall in red cells - anemia, severe sweating, 22 bronchial secretion and painful abdominal cramps,...), probably due to the high doses used (Barclay 23 and Cooke, 1945). In 1946-1948, improvement of liver functions, notably ascite clearance and 24 decreased liver size, were reported in patient with cirrhosis of the liver with ascites and that were 25 administered a low fat, high-protein/carbohydrate diet supplemented with choline (1 g daily) 26 (Broun, 1948) or a combination of choline and cystine (1-3 g daily each) (Beams, 1946). In the 
1 latter study, hepatic fatty changes were suspected based on the agreement that such treatment is

2 more effective "when there are fatty changes in the liver" and when there is an enlarged liver rather

3 than when livers are small and probably contracted by fibrous tissue: a lipotropic action of choline

4 and cystine was therefore proposed (Beams, 1946). Prolonged hepatic fatty infiltration was indeed

5 emphasized in the development of cirrhosis associated with diabetes and chronic alcoholism

6 (Russakoff and Blumberg, 1944). Latter, the positive effects of a lipotropic therapy were reported in

7 humans exhibiting various hepatic dysfunctions and/or atherosclerosis (Colson and Gallay, 1964;

8 Nadeau et al., 1954; Navarranne et al., 1964; Warembourg and Bertrand, 1964). Thus, in 1954,

9 Nadeau et al. suggested that fatty liver in alcoholic patients may result from a dietary carence that

10 has lead to choline deficiency, and they observed that the administration of lipotrope tablets lead to

11 rapid improvement of hepatic function - by decreasing values of the bromosulphalein test, this latter

12 being notably shown in dogs to be tightly related to hepatic fatty overload (Hough et al., 1943;

13 Popper and Schaffner, 1952) - and might be a significant supplement to an adequate diet (Nadeau et

14 al., 1954). In 1964, several authors reported improvements of hepatic function and atherosclerotic

15 markers in humans with hepatic and/or cardiovascular dysfunctions following admisnitration of

16 Ornitaine $^{\circledR}$ (10.045 formula, Jacques Logeais laboratory, Issy-Les-Moulineaux), a cocktail

17 containing ornithine chlorhydrate and other associated substances such as pyridoxine chlorhydrate,

18 sorbitol and 2 lipotropes that are betaine and magnesium citrate (Navarranne et al., 1964;

19 Warembourg and Bertrand, 1964). In 1991, Zeisel et al. reported that choline-deficient subjects

20 developed upon 3 weeks symptoms of incipient liver dysfunction, notably an increased in serum

21 alanin aminotransferase (ALT) and a decrease in plasma phosphatidylcholine (Zeisel et al., 1991).

22 More recently, it was shown (via the use of computed tomography, a non-invasive method for

23 estimating hepatic fat content) in patients receiving parenteral nutrition that dietary choline

24 deficiency lead to the development of hepatosteatosis, as it was reported in animal models

25 (Buchman et al., 2001; Zeisel et al., 1991). However, it was also shown that plasma level of free

26 choline and PL-bound choline were not different between patients with and without severe liver 
1 fibrosis, and was not correlated with the degree of fat infiltration within liver (Nehra et al., 2001).

2 More recently, men ( $40 \%$ of the 20 tested) and postmenopausal women $(80 \%$ of the 15 tested) 3 deprived of dietary choline have been reported to develop hepatic steatosis, the most common sign 4 of choline deficiency (Fischer et al., 2007).

Betaine has above all been used in human for treating homocystinuria that notably results 6 from a deficit in cystathionine synthase (Berlow et al., 1989). Its use in the treatment of non7 alcoholic steatohepatitis has been however shown in humans to lead to significant improvement of 8 liver functions such as a decreased in level of serum ALT during treatment and a lower degree of 9 steatosis, necroinflammatory grade and stage of fibrosis (observed via biopsies) after one year of 10 betaine treatment (Abdelmalek et al., 2001); and the use during 8 weeks of oral betaine glucuronate 11 combined with diethanolamine glucuronate (used for PL synthesis) and nicotinamide ascorbate 12 significantly reduces hepatic steatosis scores and liver enlargement in patients with non-alcoholic steatohepatitis as compared to a placebo without adverse effects (Miglio et al., 2000).

Methionine has been also early recognized as a lipotrope compound (Best and Ridout, 1940;

Caballero et al., 2008; Chahl and Kratzing, 1966b; Shils and Stewart, 1954; Tucker and Eckstein, 1937) and would directly account for the lipotropic effect of proteins (Eckstein, 1952). The lipotropic effect of methionine was demonstrated to be notably based on methyl supply for choline synthesis (see Figure 2A) (Du Vigneaud et al., 1940; Du Vigneaud et al., 1941). This was latter confirmed that methionine does not directly act upon lipid metabolism but as a precursor of choline through methyl donation to phosphatidylethanolamine (Figure 2A) (Labadie, 1974). Its lipotropic 21 potency would be weaker than that of choline at equivalent quantities (Chahl and Kratzing, 1966b), up to 3-fold lower as shown in weanling rats (Griffith and Mulford, 1941a). Methionine is also the product of homocysteine methylation by betaine (Figure 2A). Although partial deficiencies of some amino-acid (e.g. threonine) may lead to fat accumulation into rat liver (Harper et al., 1954a) and although protein play a role in controlling liver fat content (Channon and Wilkinson, 1935), only 26 methionine among the essential amino-acids appears to exert a direct lipotropic effect (Eckstein, 
1 1952). However, high doses of methionine (2.5\% of the diet) were shown to increase incorporation

2 of acetate into liver lipids $(+118 \%)$ after 7 days of treatment in rats fed a standard 9\% casein-based 3 diet (Supplemental Table 1) (Yokota et al., 1974).

4 More generally, this tends to emphasize that lipotropic effect seems to depend on the 5 lipotrope dose used whatever the compound considered and that a balanced amount of various 6 lipotropes at moderate dose might be the best equilibrium to reach - as we will discuss later.

Detailed physiological mechanisms associated with the lipotropic effect of betaine, choline,

9 methionine and myo-inositol

10 The mechanisms by which betaine, choline, myo-inositol and methionine prevent development of 11 fatty liver is mainly in relation with a facilitated transfer of FA from liver to bloodstream (Arvidson 12 and Borgström, 1963; Yagi and Kotaki, 1969), a decreased neutral lipid content in the liver (Leclerc 13 and Miller, 1989), an improvement of TG-rich lipoprotein formation (VLDL and LDL that include 14 PL) and their increased secretion from the liver (Burton and Wells, 1977; Kotaki et al., 1968; 15 Lombardi, 1971; Mookerjea, 1971; Yao and Vance, 1990; Zilversmit and Diluzio, 1958), a reduced 16 rate of FA mobilization from adipose tissue to the liver (Hayashi et al., 1974b), and/or a reduced 17 lipid synthesis in the liver by a reduced FAS and/or ACC activities (Beach and Flick, 1982; Ikeda et 18 al., 1992; Katayama, 1997b).

More generally, lipotropic effect is related to the ability for betaine, choline and methionine 20 to transfer their labile methyl groups, thus participating in a chain reaction that finally yields 21 compounds in charge of regulating fat transit outside the liver (Figure 2A) or towards mitochondria 22 where they are $\beta$-oxidized (Figure 2B) (Labadie, 1974). Myo-inositol being not a methyl donor, its 23 lipotropic effect is mainly based on its ability to favour phosphatidylinositol synthesis that is 24 thereafter used for lipoprotein formation in reticulum endoplasmic or for lipoprotein transport from 25 liver to bloodstream (Figure 2A) (Yagi and Kotaki, 1969). 
1 Thus, choline participates in and accelerates the synthesis of fat into PL from

2 phosphatidylethanolamine - notably of lecithin type like phosphatidylcholine (Figure 2A) (Mchenry

3 and Patterson, 1944; Mookerjea, 1971; Nadeau et al., 1954; Tokmakjian and Haines, 1979), this

4 latter being indispensable to export fat outside hepatocytes and methionine indirectly contribute to

5 fat exportation from liver by allowing formation of choline. Accordingly, phosphatidylcholine has

6 been shown to limit excess TG in cultured rat hepatocytes by favouring their exportation via

7 lipoproteins (Yao and Vance, 1988, 1989). As choline, betaine was early shown to accelerate PL

8 turnover but the effect would be less than choline in doses up to $50 \mathrm{mg}$ per rat and the increase not

9 directly proportional to doses ingested (Perlman and Chaikoff, 1939).

10 In culture hepatocytes from rats fed a choline-deficient diet, Yao and Vance unravelled

11 important mechanisms that are involved in the lipotropic effect of choline, betaine and methionine,

12 i.e.: normal hepatic secretion of VLDL (a TG-rich lipoprotein) requires phosphatidylcholine 13 synthesis - i.e. a choline head group moiety -, choline and methionine stimulate the synthesis of 14 phosphatidylcholine, choline favours TG excretion from hepatocytes and betaine may correct 15 VLDL secretion inhibition initiated by choline deficiency (Yao and Vance, 1988, 1989). 16 Accordingly, the impairment of lipoprotein and TG secretions from liver, the subsequent increase in 17 hepatic TG synthesis - i.e. increased activity of FAS (Rosenfeld, 1973) - and the decreased plasma 18 PL levels (lecithins and sphingomyelins) of chilomicrons, VLDL and LDL have been reported in 19 rats deprived of choline (Lombardi et al., 1968; Mookerjea, 1971; Mookerjea et al., 1975; Olson et 20 al., 1958a), TG being characterized by increased palmitic acid (16:0) content (Rosenfeld, 1973) 21 this latter being the first FA produced during lipogenesis and from which longer FA are generated. 22 In the absence of adequate phosphatidylcholine, cholesterol and TG are likely to move towards 23 cytosol, leading to fatty liver as shown in choline-deficient rats (Da Costa et al., 1995). Latter, in 24 choline-deficient rats, Yao and Vance observed hepatic TG accumulation, plasmatic TG and VLDL 25 reduction, decrease in phosphatidylcholine and TG content of VLDL but no change in plasmatic 
1 HDL level (Yao and Vance, 1990). Choline may also prevent from an increased phospholipases $\mathrm{A}_{2}$

2 and C activity, the enzymes that releases FFA from membrane PL (Singh et al., 1990).

3 To go further, KO mice for the hepatic enzyme that allow transformation of 4 phosphatidylethanolamine into phosphatidylcholine (i.e. phosphatidylethanolamine $N$ 5 methyltransferase: Pemt ${ }^{-/-}$mice) and/or for the hepatic enzyme that allow phosphatidylcholine to be 6 secreted within bile (i.e. phosphatidylcholine-specific flippase, multiple drug-resistant protein 2: $7 \mathrm{Mdr} 2^{-/ /} / \mathrm{Pemt}^{-/-}$mice) were produced by breeding (Li et al., 2005). It was clearly shown that choline8 deficient Pemt $^{-/-}$mice died within 5 days after an hepatic phosphatidyl depletion of 50\% but that 9 choline-deficient $\mathrm{Mdr}^{-/ /} / \mathrm{Pemt}^{-/-}$mice survived until more than 90 days with the same $50 \%$ 10 phosphatidylcholine depletion, effect being attributed to an important adaptation of the 11 phosphatidylcholine homeostasis that is activation of various hepatic choline recycling pathway 12 (e.g. up regulation of phospholipase $\mathrm{A}_{2}$, choline kinase and phosphocholine cytidyltransferase 13 activities and decreased expression of choline oxidase) and the lack of phosphatidylcholine 14 depletion via biliary secretion (Li et al., 2005).

Choline deficiency therefore does not allow supplying the adequate amount of PL for 16 lipoprotein synthesis and leads to impaired released of hepatic TG into plasma, to reduced levels of 17 plasma and hepatic PL and consequently to reduced lipoprotein secretion from liver (Haines and 18 Mookerje.S, 1965; Recknagel, 1967). Lipoproteins indeed include a membrane that contains PL 19 such as phosphatidylcholine (i.e. lecithin) to the formation of which participate choline, but also 20 myo-inositol (Mchenry and Patterson, 1944; Yagi and Kotaki, 1969). However, by using germ-free 21 and inositol-deficient mice, it was demonstrated that inositol synthetized by intestinal microflora do 22 not contribute to reduce the extent of fatty liver (Ikeda et al., 1992). Same authors showed that 23 inositol may also depress the activity of several enzymes involved in hepatic lipogenesis, i.e. FAS, 24 G6PDH (Glucose-6-phosphate dehydrogenase) and ACC (Ikeda et al., 1992). Since the effect of 25 inositol supplementation on decreasing these enzyme activities was less marked, their results would 
1 also suggested that a fraction of dietary inositol may be degraded or used for fuel by microbiota at

2 the intestinal level (Ikeda et al., 1992).

In the end, another unexpected cellular mechanism might be involved in the lipotropic effect

4 of betaine, choline and myo-insoitol. Indeed, as small hydrosoluble molecules that do not interfere

5 with cellular protein functions - even at high concentrations -, betaine, choline and myo-inositol are

6 all osmolytes and may participate in cell volume regulation, the level of cellular hydration affecting

7 cellular metabolism via gene expression modifications (Häussinger, 1996). Thus, increased cell

8 swelling in rat hepatocytes was shown to increase lipogenesis and to activate ACC (Baquet et al.,

9 1991; Hue, 1994), this enzyme allowing formation of the metabolic intermediate malonyl-CoA that

10 plays a major role in FA synthesis. In the same way, hypo-osmotic incubation of hepatocytes - i.e.

11 that increases their volume - was shown to inhibits CPT-1 (carnitine palmitoyltransferase-1) (allows

12 lipid transfer within mitochondria) whose deficit lead to defective FA oxidation (Figure 2B)

13 (Guzmán et al., 1994). Conversely, transfert of osmolytes into cell will lead to cell shrinkage and 14 inverse effects (Häussinger, 1996). We may therefore hypothesized that increased cellular content

15 of betaine, choline and myo-inositol might contribute to cell shrinkage with possible potential 16 positive effects upon lipid metabolism and fat liver content (Figure 2A).

18 Lipotropes or methyl donors?

19 It has been reported that lipotrope-deficient diets may be carcinogenic in the absence of carcinogens 20 (Henning and Swendseid, 1996,Moon et al., 1998,Poirier and Whitehead, 1973): this is why 21 lipotrope-deficient diets have often been used to favour carcinogenesis in rats (Rogers, 1975), more 22 specifically in liver (Christman et al., 1993). This is based on the property of some lipotropes to 23 transfer their methyl groups (labile methyls) and on the association between an increased level of 24 DNA hypomethylation and cancers (Goelz et al., 1985; Van Den Veyver, 2002) as it was shown in 25 rats consuming lipotrope-deficient diets (Christman et al., 1993; Locker et al., 1986). For exemple, 26 female rats fed a methyl-deficient diet and in which mammary carcinogenesis was induced were 
1 also characterized by DNA hypomethylation in mammary tissues that was associated with the

2 highest number of tumors (Moon et al., 1998). More generally, a decrease in the amount of methyl

3 groups within organism would favour an increased sensibility towards cancers by altering immune

4 function and xenobiotic (e.g. carcinogens) metabolism (Nauss et al., 1982; Newberne and Rogers, 5 1986).

6 The lipotrope/methyl donor-deficient diet is therefore the only dietary deficiency to be 7 carcinogenic (Ghoshal and Farber, 1984; Locker et al., 1986; Wu et al., 1998). Maybe this is one of 8 the reasons why both lipotrope- and methyl donor-deficiencies have been, purposely or not, often 9 confounded until now (Wu et al., 1998). The term methyl donor-deficient diet is today most often 10 used than lipotrope-deficient diet. Yet, while all lipotropes are not methyl donors (e.g. myo11 inositol), all methyl donors have not been shown to be lipotropic (e.g. S-adenosyl-methionine).

14 Are proteins lipotropic? \{Zhang, 1993 \#26561\}: fish proteins and cholesterol in rats

18 and linked to protein metabolism (Best and Huntsman, 1935). Thus, the same year, it was shown 19 that increasing the protein content (caseinogen, from 0 to $50 \%$ ) of a high-fat diet (40\%) containing $2017.5 \mathrm{mg}$ choline $/ 100 \mathrm{~g}$ at the expense of carbohydrates (glucose hydrate, from 50 to $0 \%$ ) 21 counteracted the development of fatty liver in rats, and the effect was apparently dose-dependent 22 (Channon and Wilkinson, 1935). In the same study, authors also showed that the quality of liver 23 lipids was altered upon high-protein diet with phosphatide and free cholesterol percentage 24 increasing while TG percentage decreasing; and they finally suggested that some aminoacids of 25 caseinogen may be converted within choline and betaine (Channon and Wilkinson, 1935). Their 26 results were further criticized by Best et al. that found higher liver fat percentages in rats within 
1 similar conditions of diet and they argued that their diet would contain other non-protein "lipotropic

2 factor" (Best, 1935). The lack of an adequate amount of protein in the diet was however latter

3 shown to cause hepatic fat accumulation in rats by these same authors (Best et al., 1955); however,

4 re-feeding rats with an adequate diet containing $18 \%$ casein lead to the development of a "transient"

5 increased fatty liver that return to normality after 3 weeks of the diet (Best et al., 1955).

Based on the previously demonstrated lipotropic effect of betaine and choline (Best and

7 Huntsman, 1932), it was hypothesized that amino-acids from casein were converted into betaine and choline in the liver (Channon and Wilkinson, 1935). One thereafter wondered which aminoacid was more particularly involved in the lipotropic effect of proteins. Methionine was thus rapidly shown to be lipotropic while cystine supplementation by $0.5 \%$ in the diet increased fat liver content 11 in rats (Beeston and Channon, 1936; Tucker and Eckstein, 1937) and lysine had no effect (Tucker

and Eckstein, 1938). In addition to the lipotropic effect of methionine from casein, that of threonine

13 was also suggested (Beveridge et al., 1945) then confirmed (Harper et al., 1953) but partly 14 depending on the amount of tryptophane, glycine or protein in the diet (Harper et al., 1954b,Singal cholesterol-liver, not with high-FA-liver - was otherwise reported (Channon et al., 1943). However, except methionine, it was also observed in rats a lack of lipotropic effect for all essential amino18 acids including threonine (Eckstein, 1952).

A series of proteins was also tested for their lipotropic activity and the following ranking 20 was obtained by deceasing intensity: gromax and whale muscle protein $>$ caseinogen $>$ albumin $>$ 21 beef muscle protein and edestin > fibrin and gliadin > gelatine and zein (Channon et al., 1938); and 22 it was noted that the lipotropic effect of these proteins correlated with their methionine content 23 (Tucker and Eckstein, 1938). Thus, arachin, a protein of low methionine content had no lipotropic 24 activity (Singal and Eckstein, 1939).

In 1969, it was simply demonstrated that rats fed a low-protein diet (5\% casein only) had a 26 higher hepatic total FA content compared to normal diet - together with a lower level of liver PL of 
$127 \%$ after 6 weeks (Osumi et al., 1969). The lipotropic action of proteins was further underlined in

2 rats and woodchucks for which the effect of lipotropic factors (choline, methionine, folic acid and

3 vitamin B12) varied according to the amount of soy protein isolate in the diet (i.e. 10 vs $20 \%$ ) Study by Iritani et al. (1986): lipotropic effect of gluten and soybean protein vs casein and fish protein towards TG content in liver

94 (Boyd et al., 1986). Lipotropic effect of proteins has also been emphasized in rats fed a high-fat plus

5 cholesterol vs high-fat and fish proteins plus cholesterol diet, results showing a significantly lower

6 level of hepatic total cholesterol and TG with the fish protein-containing diet (Hosomi et al., 2009).

7 Both an increased expression of hepatic CYP7A1 (cholesterol $7 \alpha$-hydroxylase) - via activation of 8 the transcription factor liver receptor homologue-1 - and an inhibition of cholesterol and bile acid

9 absorption within small intestine are notably involved (Hosomi et al., 2009). Similarly, compared to

10 casein, rats fed pork protein had lower hepatic levels of TG (-46\%) via decreased mRNA SREBP-

11 1c (sterol regulatory element binding protein) and G6PDH concentrations, i.e. via a reduced FA

12 synthesis (Brandsch et al., 2006). In rats receiving cholesterol intravenously and intragastrically,

13 soy protein compared to casein was shown to be antihypercholesterolemic via stimulation of hepatic

14 cholesterol synthesis in response to increased faecal steroid excretion (Nagata et al., 1982). Such an

15 effect may be attributable to the lower digestibility of soybean protein compared to casein,

16 hydrophobic peptides of soybean protein binding bile acids and consequently stimulating hepatic

17 cholesterol turnover (Iwami et al., 1986). Moreover, it was shown in rats that highly purified 18 soybean proteins affect enzymes involved in cholesterol metabolism (Madani et al., 1998). 19 Compared to casein, rice and soy proteins were also shown to exert lipotropic effect in both 20 growing and adults rats fed or not with high-cholesterol diet, protective mechanisms involving a 21 reduced secretion of hepatic cholesterol into circulation, an increased excretion of biliary bile acids 22 and reduced hepatic TG accumulation (Yang and Kadowaki, 2009; Yang et al., 2007). Compared to 23 casein, soybean proteins were also shown to significantly reduce cholesterol, TG and ApoA-1 24 (apolipoprotein A-1) secretion from isolated rat liver, and cholesterol and TG contents in liver; 60

26 (Sugano et al., 1982). 
Lipotropic effect of proteins seems therefore to depend on protein origin - and probably also

2 methionine content. Thus, in rats fed $25 \%$ either casein or proteins from lactalbumin, whole egg,

3 egg albumin, sardine, soybean and wheat gluten, its has been shown significant variations in hepatic

4 cholesterol, TG and PL concentrations, wheat gluten proteins leading to the highest lipid

5 accumulation while soybean proteins leading to the lowest TG accumulation (Sugiyama et al.,

6 1996). In addition, authors reported that lactalbumine and whole egg proteins lead to the highest

7 methionine concentration in rat liver, that casein lead to around $10 \%$ more phosphatidylcholine

8 relative to total PL than soybean proteins, and that methionine content of dietary proteins was

9 correlated with the liver microsomal phosphatidylcholine/phosphatidylethanolamine ratio

10 (Sugiyama et al., 1996).

11 In humans, the lipotropic effect of proteins was apparently very little studied. A report was 12 made with a mildly hypercholeterolemic and healthy middle-aged alcoholic woman upon either a 13 normal diet containing $100 \mathrm{~g}$ protein or a low-protein diet of $25 \mathrm{~g}$ : liver biopsies did not reveal any 14 fatty material accumulation upon the low-protein diet but it was observed in serum important 15 decreases in lipid (cholesterol, PL and TG) and lipoprotein concentrations suggesting impairment of 16 lipid metabolism within liver, notably for cholesterol (Olson et al., 1958b). Then the administration 17 of a supplement of lipotropic factors (choline, methionine, inositol, vitamin B12 and liver 18 concentrate) restaured serum cholesterol to its normal level (Olson et al., 1958b). The lipotropic 19 effect of proteins has been recently confirmed in healthy humans fed a high-fat vs a high-fat and 20 high-animal protein diet by measuring the intrahepatocellular lipids by ${ }^{1} \mathrm{H}$-magnetic resonance 21 spectroscopy: a blunting effect of proteins upon liver lipids $(\approx-22 \%)$ was observed (Bortolotti et al., 22 2009) The lipotropic effect of caloric restriction (30\%) in humans \{Elias, $2010 \# 25149\}$ $\{$ Lazo, 2010 \#22481\}: Reduced steatosis through better lifestyle (moderate caloric restriction + exercise) is also possible : another alternative to lipotropes or a combination of both. 
1 Besides myo-inositol, inositol possess 8 other isomers, notably chiro- and scyllo-inositol that are

2 also present in PBF, but at a largely lower levels than myo-inositol (Kim et al., 2005; Sanz et al., 3 2004). However, to our knowledge, only myo-inositol was shown to have lipotropic properties 4 (Andersen and Holub, 1980; Beach and Flick, 1982; Okazaki et al., 2006; Yagi and Kotaki, 1969). 5 Conversely, chiro-inositol consumption has been reported to increase fat deposits in rat liver 6 (Okazaki et al., 2006). Actually, chiro-inositol is recognized for its ability to improve insulin 7 regulation and is used in diabetes management (Kim et al., 2005).

Myo-inositol is present in PBF mainly as free or conjugated forms such as galactinol (i.e. 9 monoglycosylated myo-inositol), di-glycosylated myo-inositol (Horbowicz et al., 1998, 10 al., 1982,Steadman et al., 2000) or myo-inositol phosphates such as myo-inositol hexakisphosphate 11 (i.e. IP6) or phytic acid that is generally the most abundant myo-inositol phosphate followed by IP5, 12 IP4, etc. (Chen, 2004; Helfrich and Bettmer, 2004). However, as regards with high phytic acid 13 content in numerous PBF, especially grain products - i.e. whole-grain cereals, legumes, nuts and 14 seeds -, the question whether or not phytic acid has to be considered as a source of lipotropes is an 15 important issue.

Phytic acid has been reported to reduce hepatic and serum lipid levels in diabetic and aged 17 ICR mice (Lee et al., 2005; Lee et al., 2007b), in high-sucrose fed rats (Katayama, 1995; Onomi et 18 al., 2004) and in DDT-fed rats (Okazaki et al., 2003) via notably a significant increase in fecal 19 triacylglycerols, cholesterol and bile acid contents (Lee et al., 2007b) (Supplemental Table 3). A 20 decreased dose-dependent effect on several hepatic lipid parameters (total lipids and TG contents, 21 and G6PDH, malic enzyme - ME - and FAS activities) was otherwise shown in high-sucrose fed 22 rats with increasing level of phytae from 0.1 to $2.5 \%$ of the diet (Katayama, 1997a). Mechanisms 23 involve a depressed activity of lipogenic enzymes such as FAS and NADPH-generating enzymes 24 NADPH being importantly used for FA synthesis - like ME, G6PDH and 6-phosphogluconate 25 dehydrogenase (Katayama, 1995,Okazaki et al., 2003,Onomi et al., 2004). Phytic acid was also 26 shown to have a similar lipotropic action than free myo-inositol in sucrose-fed rats in relation with a 
1 decreased hepatic lipogenesis (Katayama, 1997b). Interestingly, hepatic free myo-inositol content

2 was identical for rats fed either phytic acid or free myo-inositol (Okazaki and Katayama, 2008),

3 suggesting a metabolisation of phytic acid in rats and mice. This is probably the result of phytate

4 hydrolysis into free myo-inositol by small intestine phytases through an adaptative response before

5 phytic acid be fermented within the colon (Lopez et al., 2002; Lopez et al., 2000). Accordingly, it

6 has been previously shown that phytic acid is rapidly absorbed in stomach and small intestine of

7 rats, and then metabolized and distributed to various tissues, probably mainly under the form of myo-inositol and/or IP1 (Sakamoto et al., 1993). However, no studies reported lipotropic effect of

9 phytate in humans. This has probably to be related to the weaker phytase activity in humans which

10 is reported to be 30 -fold less than in rat duodenum (Iqbal et al., 1994).

Yet, phytate was shown very early to be degraded in humans based on a $20-60 \%$ recovery of 12 ingested phytin (calcium-magnesium salt of phytate) in faeces (McCance and Widdowson, 1935). A $1360 \%$ degradation of wheat bran phytate into myo-inositol penta-, tetra- and triphosphates has also 14 been reported in ileostomates (Sandberg et al., 1987). Although mucosal phytases and alkaline 15 phosphatases are present in humans (Bitar and Reinhold, 1972), the degradation of phytate appears 16 to be mainly due to dietary phytases of plant and/or microbial origins that could be activated at the 17 low $\mathrm{pH}$ encountered in the stomach (as e.g. for cereal phytases), as shown in healthy ileostomates 18 with phytase-deactivated wheat bran (Sandberg and Andersson, 1988), but also due to endogenous 19 microbial phytases within the colon (Sandberg and Andlid, 2002). Another in vitro study lead 20 within 3 cell lines (i.e. mouse $\mathrm{T}$ cell leukemia, human erythroleukemia and human colon 21 adenocarcinoma) showed that phytic acid may be uptook as such and/or partly dephosphorylated 22 (Vucenik and Shamsuddin, 1994). However, no human studies have reported increased hepatic free 23 myo-inositol content or improved liver FA metabolism following high-phytate consumption. Up 24 today, results are therefore not sufficiently convincing to consider myo-inositol phosphates as a 25 source of lipotropes in humans; and the extrapolation of the lipotropic effect of myo-inositol 26 phosphates from rats to human remains highly uncertain or prematured. 


\section{The lipotropic effect of carnitine}

Study in humans with carnitine: no effect \{Uygun, 2000 \#17953\} Home parenteral nutrition and carnitine deficiency: a probable cause for steatosis \{Bowyer, $1985 \# 17975$ \}

6 lipotropic effect in rats fed choline-methionine-deficient and high-fat (30\%) diet (Khairallah and

7 Wolf, 1965), or in rats submitted to protein and/or methionine/lysine (carnitine precursors)

restrictions (Hu, 1975,Ortega, 1989). The effect is dose-dependent between 0.1 and $0.8 \%$ of the diet and apparently more marked with TG than with other classes of lipids that are PL, cholesterol and nonesterified FA (Supplemental Table 2) (Rhew and Sachan, 1986). Indeed, carnitine was shown to increase hepatic cholesterol content in sedentary vs excersized rats fed high-fat diet rich in either saturated or monounsaturated FA, probably as a result of an increased cholesterol turnover (Karanth and Jeevaratnam, 2009); and in obese cats, high level of carnitine in the diet (1000 mg/kg) did not reduce liver lipid (TG, PL and cholesterol) contents compared to low level (40 mg/kg) (Blanchard et al., 2002).

As choline, betaine and myo-inositol, the lipotropic effect of carnitine can be also simply unravelled in carnitine-deficient rats that notably develop an important increase in hepatic TG content $(>+250 \%)$ and a significant decrease in PL content (-22 and $-36 \%$; Supplemental Table 2) (Degrace et al., 2007). However, at equimolar amounts, lipotropic effect of carnitine was demonstrated to be significantly lower in rat than that of choline (Hu, 1975; Khairallah and Wolf, 1965). In addition, in rats fed a 20\%-protein and choline deficient diet, carnitine surprisingly did not allow preventing fatty liver whereas choline did, probably since methyl group of carnitine is not labile and cannot be transferred to form methionine from homocysteine (Fritz and Dupont, 1957). 
1 Carnitine is mandatory for the uptake of long-chain FA acyl-CoA from the cytosol to

2 mitochondria where they are $\beta$-oxidized to produce energy (Figure 2B). Accordingly, carnitine

3 acyltransferase, the rate-limiting enzyme in FA $\beta$-oxidation is activated by exogenous carnitine

4 (Mccarty, 1994). In humans, it is proposed as commercial fat burners to help loose weight through

5 increased fat oxidation rate as shown in overweight subjects (Wutzke and Lorenz, 2004), but also to

6 increase exercise performances (Decombaz et al., 1992; Lennon et al., 1983). The lipotropic effect

7 of carnitine is therefore to stimulate FA oxidation $(\mathrm{Hu}, 1975)$.

Body carnitine results from both synthesis from dietary lysine and methionine contents

9 (Figure 2C) and from natural carnitine found in low amount in PBF such as avocado, tempeh

10 (fermented soya), some nuts, seeds, legumes, vegetables, fruits et cereals (e.g. pumpkin, sunflower,

11 sesame, cabbage, common bean, apricots and banana). Compared to animal tissues, the carnitine

12 and acylcarnitine ( $2 \%$ of the total carnitine pool) contents in plant tissues is around a hundred and 13 thousand times lower (Bourdin et al., 2007) and best sources are of animal origin such as red meat 14 and, to a lesser extent, milk products (Seline and Johein, 2007). Values of respectively 0.32, 0.51 15 and $0.27 \mathrm{mg} / 100 \mathrm{~g}$ dry weight $(\mathrm{dw})$ have been reported for rapessed, flax and tobacco (Bourdin et 16 al., 2007), values that are closer to ranges found for B vitamins in PBF than those found for betaine, 17 choline, myo-inositol and methionine. More generally, Seline and Johein determined total carnitine 18 contents of 74 food products and obtained the following ranges on a fresh weight-basis: 3.2 (breast 19 pheasant) - 166.0 (kangaroo steak) mg/100 g for 20 animal products, 0.64 (Babybel $\left.{ }^{\circledR}\right)-14.9$ 20 (Norwegian goat cheese) $\mathrm{mg} / 100 \mathrm{~g}$ for 20 cheeses, 2.2 (yogurt) - 42.8 (condensed milk) mg/100 g 21 for 17 liquid dairy products, and 0.014 (orange) - 4.98 (oyster mushroom) mg/100 g for 13 plant22 based foods (Seline and Johein, 2007), thus confirming conclusions of Bourdin et al. when 23 comparing animal- and plant-based foods (Bourdin et al., 2007). Among PBF, mushrooms (1.32, 242.62 and $4.98 \mathrm{mg} / 100 \mathrm{~g}$ for respectively chanterelle, mushroom and oyster mushroom) appears as 25 the best source of carnitine both on a $100 \mathrm{~g}$ fresh food- and dry weight-basis followed by avocado 
1 (0.43 mg/100 g), carrot $(0.40 \mathrm{mg} / 100 \mathrm{~g})$, cauliflower $(0.36 \mathrm{mg} / 100 \mathrm{~g})$, cucumber $(0.19 \mathrm{mg} / 100 \mathrm{~g})$, 2 banana $(0.10 \mathrm{mg} / 100 \mathrm{~g})$ and apple $(0.05 \mathrm{mg} / 100 \mathrm{~g})$ (Seline and Johein, 2007)

3

4

5

6

7 Magnesium and B vitamins

\section{The contribution of magnesium and vitamins B to the overall lipotropic effect}

8 In addition to the well-recognized lipotrope compounds that are choline, myo-inositol, methionine

9 and betaine, the contribution of micronutrients such as niacin (vitamin B3) (Perry, 1960, Van Der

10 Hoorn et al., 2008), pantothenic acid (vitamin B5) (Catolla Cavalcanti and Levis, 1950; Turchetto et al., 1955), folates (vitamin B9) (Kelley et al., 1950; Laird and Drill, 1971) and magnesium (Colson and Gallay, 1964; Navarranne et al., 1964; Warembourg and Bertrand, 1964) to the overall

13 lipotropic effect of PBF has been also emphasized (Supplemental Table 1). Although very early shown to exert a lipotrope effect in rats (Halliday, 1938), pyridoxin (vitamin B6) was no longer considered as a lipotrope (Carter and Phizackerley, 1951) due to further contradictory results (Audet and Lupien, 1974; Gavin and Mchenry, 1940; Johnston et al., 1961; Mchenry and Gavin, 1941; Saheb and Demers, 1972); and the lipotropic effect of pyridoxin has not been convincingly confirmed until today despite several studies showing the development of fatty liver in rats fed a high protein diet without pyridoxin (Abe and Kishino, 1982; Okada and Ochi, 1971; Okada and Suzuki, 1974; Suzuki et al., 1976). Therefore, although some have considered it as a lipotrope and although it is used within the composition of commercial lipotrope supplements, one believes that literature is not enough convincing to validate it as a lipotrope, especially in humans.

Lipotrope effect has also been reported for vitamin B12 (cobalamine) either alone (Drill, 1954; Quan and Le Breton, 1973; St. Greif and Wenning, 1954; Shils and Stewart, 1954) or in combination with choline and folates (Laird and Drill, 1971), this B vitamin being only present in animal-based food (ABF) products, and to a lesser extent in some fermented cereals (e.g. beer) 
1 where it is supplied by yeast. It is notably involved within the process of transmethylation that

2 corresponds to the transfert of a methyl group from 5-methyl tetrahydrofolates to homocysteine 3 allowing methionine formation in a way similar to the action of betaine with homocysteine (Figure 4 2A) (Gillis and Norris, 1951; Jaenicke and Rudiger, 1971; Newberne and Rogers, 1986). 5 Accordingly, carcinogenic lipotrope-deficient or methyl donor-deficient diets generally include 6 vitamin B12 deficiency (Christman et al., 1993; Moon et al., 1998; Newberne et al., 1971; Rogers 7 and Newberne, 1969; Wu et al., 1998).

9 Physiological mechanisms associated with the lipotropic effect of B vitamins and magnesium

10 The mechanisms by which magnesium and B vitamins may limit fat deposits are multi-factorial, 11 especially for niacin.

13 Folates (vitamin B9)

14 For folates (or folic acid), the mechanism involved in its contribution to the overall lipotropic effect 15 is its action as precursor of the methyl donor 5-methyl tetrahydrofolate that leads to methionine 16 formation from homocysteine via methyl donation, and latter to choline regeneration (Figure 2A) 17 (Zeisel, 1981), thus importantly participating in the lipotropic effect. Thus, it has been shown in 18 chronically ethanol fed micropigs that folate deficiency accelerated alcoholic steatosis as shown by 19 liver histopathology and by accentuation of abnormal methionine metabolism (i.e. hepatic 20 methionine depletion were of -39 and $-68 \%$ for respectively folates-sufficient and folate-depleted $v s$ 21 nopn-alcoholic and folate-sufficient micropigs; Supplemental Table 1) (Halsted et al., 2002). Latter, 22 the same research team showed that folate deficiency was also accompanied by significant effects 23 on gene expression in relation with lipid metabolism, notably an increased mRNA expression of 24 SREBP-1c and ACC (key compounds in lipogenesis) - but no effect on FAS mRNA expression - in chronically ethanol or not fed micropigs (Supplemental Table 1) (Esfandiari et al., 2005). 26 lipotropic effect of folic acid has been also emphasized in rats (Drill, 1954; Kelley et al., 1950), but 
1 it appears to be effective only when adequate amounts of other lipotropes, notably choline, are

2 initially present in the diet (Laird et al., 1965). This supportive lipotropic effect of folates is 3 concomitant with their ability to reduce hyperhomocysteinemia (Brouwer et al., 1999; Moat et al., 4 2003), a CVD risk factor.

$6 \quad$ Niacin (vitamin B3)

7 Although we chose to consider niacin (vitamin B3 or vitamin PP or nicotinic acid) as contributing 8 to the overall lipotropic effect of PBF, first reported results were quite contradictory as regards with 9 effect of this vitamin B on hepatic lipid metabolism (Baker et al., 1977; Baker et al., 1973; Gaylor 10 et al., 1960; Griffith and Mulford, 1941b; Merrill and Lemley-Stone, 1957; Orbetsova et al., 1977; 11 Rikans et al., 1965): for example, Griffith and Mulford observed an increased liver fat percentage of 12 around $4 \%$ in rats fed during 8 days a niacin-supplemented diet (22.3\% fat) as compared to basal 13 diet ( $18 \%$ fat), the increased range being more marked in the presence of $0.04 \%$ choline chloride 14 i.e. from 12.5 to $19.9 \%$ (Griffith and Mulford, 1941b); in addition, a 2\%-supplementation nicotinic 15 acid was shown to induce fatty liver in rats, the effect being counteracted when adding $0.4 \%$ choline 16 chloride (Handler and Dann, 1942); and a daily injection during one month of a high dose of 17 nicotinic acid (250 mg/kg b.w.) in spontaneously hypertensive rats fed either normal diet or 18 atherogenic diet ( $2 \%$ cholesterol) increased hepatic cholesterol, TG, total lipid, and esterified and 19 FFA contents (Orbetsova et al., 1977). Conversely, Merrill and Lemley-Stone latter showed that the 20 addition of $0.4 \%$ nicotinic acid to an initial $2 \%$-cholesterol diet largely lowered average liver 21 cholesterol content in rabbits from 6.55 to $1.51 \%$ (Merrill and Lemley-Stone, 1957). In 1958, Schön 22 showed that incorporation of 3-4\% nicotinic acid in a hypolipotropic diet free from cholesterol 23 partly reversed increased hepatic cholesterol concentration by around $42-46 \%$ in rats, advancing 24 that a relative lack of Coenzyme A (CoA) may be responsible for the effect of the hypolipotropic 25 diet (Schön, 1958). Then, Baker et al. showed that nicotinic acid may prevent hepatic steatosis 26 (decreased total fat, neutral fat and non-esterified FA levels to the normality) in ethanol-treated rats 
1 and hypothesized that nicotinic acid may have depress the mobilization of non-esterified FA from

2 adipose tissue that was induced by ethanol (Baker et al., 1973). Nicotinic acid was also shown to 3 importantly reduce different lipid fractions (total lipids, cholesterol, lipid phosphorus and TG) in rat 4 fatty livers induced with $\mathrm{CCl}_{4}$ and orotic acid: competitiveness with $\mathrm{CoA}$ synthesis (involved in 5 lipogenesis) and a possible inhibition of fat depot mobilization and TG/FFA availability for lipid 6 synthesis have been hypothesized in this study (Vaishwanar et al., 1972). And in laying hens 7 supplemented with niacin, $50 \mathrm{mg}$ niacin $/ \mathrm{kg}$ reduced fat infiltration in liver by around $29 \%$, but effect was not significant due to a high variability in data (Hartfiel and Kirchner, 1973). Conversely,

9 excess fat deposits in high-fat- or normal-diet-fed rats supplemented with niacin at a high level of $10 \quad 0.1 \%$ have been observed despite the presence of choline (Baker et al., 1977; Rikans et al., 1965). In 11 the study of Baker et al., the $0.1 \%$ niacin-supplementation of rats fed a choline-deficient diet lead to $12-40,+94,-14,+116$ and $+33 \%$ changes in respectively hepatic PL, TG, free cholesterol, cholesterol 13 esters and non-esterified FA contents but effects were not significant (Baker et al., 1977). Adding $140.5 \%$ choline dihydrogen citrate to the $0.1 \%$ nicotinic acid lead to reduction for all lipid classes, 15 effect becoming significant for TG (-26\%) and cholesterol ester (-7\%) contents, but surprisingly 16 also for PL content (-52\%), also indicating "that niacin interferes with choline-induced lipotropism" 17 (Baker et al., 1977). Although 0.1\% niacin-supplementation was not nutritionally realistic, Baker et 18 al. interestingly showed by using the in vitro models Escherichia coli (requiring vitamin B12 or 19 methionine) and thermophilic yeast Torulopsis pintolopessi (requiring choline or methionine) that 20 the potentiated hepatic steatosis induced by high doses of nicotinic acid (Sorrell et al., 1976) 21 although plasma TG level is generally decreased - may be ascribed to its interference in the 22 transmethylation process by preventing methionine to provide methyl groups for choline synthesis 23 and by blocking vitamin B12 from acting as a co-factor in the methylation of homocysteine in 24 methionine (Baker et al., 1977; Rikans et al., 1964). Accordingly, it had been previously 25 hypothesized that the antilipotropic effect of nicotinic acid at high doses (from 1 to $4 \%$ ) might be 26 due to the important need in methyl groups of its detoxification products (Schön, 1958) - notably 
1 nicotinamide that requires more methyl groups for excretion than nicotinic acid (Miller et al., 1960),

2 excess niacin being methylated in the liver to $N$-methyl-nicotinamide then excreted in urine 3 (Institute of Medicine, 1998); which lead to assimilate nicotinic acid to a "methyl trap that drains

4 off methyl groups from choline and/or methionine synthesis leading to a functional choline 5 deficiency" and leading to impaired secretion of lipids from the liver (Baker et al., 1977; Cantoni, 6 1951,Handler, 1944; Perlzweig et al., 1943). Indeed, addition of choline generally reverses the fatty $7 \quad$ liver induced by excess niacin (Baker et al., 1977; Rikans et al., 1965).

More specifically, concerning liver cholesterol, nicotinic acid has been shown by different 9 authors to significantly reduce its content and its rate of biosynthesis (Merrill and Lemley-Stone, 10 1957; Perry, 1960; Schade and Saltman, 1959; Schön, 1958), an effect attributed to a lack of acetyl11 CoA needed for cholesterol synthesis, CoA competing with detoxication systems - notably towards 12 nicotinuric acid at high doses of nicotinic acid - and lipid synthesis (Schade and Saltman, 1959). 13 Other advanced that nicotinic acid would divert cholesterol precursors towards oxidation rather than 14 in the cholesterol synthesis pathway, as for FA formation (Perry, 1960). In another study, different 15 rate of acetate incorporation into cholesterol synthesis were obtained with rat liver slices incubated 16 in $2-\mathrm{C}^{14}$ sodium acetate according to the mode of administration of nicotinic acid, either chronically 17 injected in rats during 21 days before killing at a level of $20 \mathrm{mg} / \mathrm{kg} \mathrm{b.w.} \mathrm{or} \mathrm{directly} \mathrm{added} \mathrm{to}$ 18 incubation medium of liver slices at a concentration of $10^{-3} \mathrm{M}$ (Orbetsova et al., 1976). In the 19 former case no changes were observed while a stimulation of acetate incorporation was reported in 20 the latter case. Authors suggested that chronic administration of nicotinic acid vs direct incubation 21 or single injection would not influence cholesterol synthesis at the same level of the metabolic chain 22 (Orbetsova et al., 1976). Accordingly, they observed in rats injected with nicotinic acid (250 $\mathrm{mg} / \mathrm{kg}$ 23 b.w.) a decreased hepatic cholesterol and TG content after 6 hours with increase after 3 hours 24 (Orbetsova, 1977). In humans, nicotinic acid administration - from 1 to $2 \mathrm{~g} 3$ times daily - lead to 25 lowered serum cholesterol levels (Miller et al., 1960; Parsons, 1961b), such reduction being likely 26 to partly result from marked reduction in hepatic cholesterol synthesis (Parsons, 1961b). Thus, from 
1 these studies, it seems that nicotinic acid induces fatty liver only at high doses and in absence of one

2 or more other lipotropes with variations according to animal species and modes of administration,

3 i.e. single injection vs chronic administration. That would partly explain apparent contradictory

4 results between studies.

Other mechanisms might be involved in the positive effect of niacin on hepatic lipid 6 metabolism. In vitro, nicotinic acid has been thus shown to importantly inhibit at various doses 7 (from 19 to $100 \%$ for respectively 10 to 100 mkmoles of nicotinic acid) ACC activity, the main enzyme involved in FA synthesis (Fomenko et al., 1979). Yet, with the objective of unravelling mechanisms by which nicotinic acid inhibits ketogenesis, when incubating in vitro mitochondria with palmitic acid, CoA, carnitine and nicotinic acid, this latter had no influence on the rate of $\beta$ oxidation, suggesting that enzymes required for palmitate $\beta$-oxidation and the production of acetyl

12 CoA are not affected by nicotinic acid (Yeh, 1976). This would confirm previous results showing 13 lack of effect of nicotinic acid on hepatic acetyl-CoA concentration at an injection level of 50 $14 \mathrm{mg} / \mathrm{kg}$ body weight (Mayor et al., 1967). Based on the antioxidant property of copper $(\mathrm{Cu})$ and of 15 the hypolipidemic capacity of niacin, Salama et al. interestingly demonstrated in high-carbohydrate fed rats that a copper nicotinic acid complex (a therapeutic drug), administered by stomach tubing at apparently nutritional doses - i.e. $400 \mathrm{mg} / \mathrm{kg}$-, is able to correct fatty liver by notably significantly 18 decreasing total lipid content and increasing antioxidant status (Salama et al., 2007), increased 19 oxidative stress via accumulation of free radicals being a cause that may lead to fatty liver. Indeed, 20 a decreased expression of superoxide dismutase has been observed in patients with cirrhotic stage 21 non-alcoholic steatohepatitis (Sreekumar et al., 2001). Such a decrease generally lead to increased 22 levels of reactive oxygen species (ROS) that may yield mutation in mitochondrial DNA, 23 mitochondria being the site of FA $\beta$-oxidation (Sreekumar et al., 2001). Finally, niacin, together 24 with pyridoxin, vitamin C, iron and other enzymes, participates in the synthesis of the lipotrope carnitine (Figure 2C). 
$1 \quad$ Recent studies allowed unravelling new mechanisms that may contribute to the overall 2 positive effect of niacin on hepatic lipid metabolism (Figure 2C). Thus, results obtained with 3 HepG2 cells showed that niacin may: $1^{\circ}$ ) inhibit TG production and FA synthesis combined with 4 accelerated ApoB (a TG-rich lipoprotein) degradation (Jin et al., 1999; Jin et al., 1996; Kashyap et 5 al., 1997; Van Der Hoorn et al., 2008); $2^{\circ}$ ) increase efflux of HDL ApoA-1 (Jin et al., 1997); $3^{\circ}$ ) 6 reduce intracellular cholesterol (total, free and esters); $4^{\circ}$ ) induce expression of PPAR $\alpha$ mRNA 7 (PPAR $\alpha$ regulates FA oxidation and stimulates peroxysome proliferation) (Siripurkpong and Na8 Bangehang, 2009); $5^{\circ}$ ) up-regulate ABCA1 (ATP-Binding Cassette Transporter 1) mRNA 9 expression (Siripurkpong and Na-Bangehang, 2009) - ABCA1 effluxes excess cellular cholesterol 10 to ApoA-1 to form nascent HDL; $6^{\circ}$ ) reduce expression of CETP (Cholesteryl Ester Transfer 11 Protein) mRNA (Van Der Hoorn et al., 2008) - CETP mediates the transfer of cholesteryl esters 12 from HDL to pro-atherogenic apoB-lipoproteins; $7^{\circ}$ ) inhibit hepatocyte DGAT (diacylglycerol 13 acyltransferase), the key enzyme for the synthesis of triglycerides, finally resulting in a potential 14 reduction of hepatic atherogenic lipoprotein secretion (Ganji et al., 2002); and $8^{\circ}$ ) inhibit surface 15 expression of ATP synthase $\beta$ chain - this latter mediating hepatic HDL endocytose (Martinez et al., 16 2003); and consequently $9^{\circ}$ ) reduce HDL uptake by HepG2 cell (Zhang et al., 2008).

In the fifties, Niacin was otherwise reported to be hypolipidemic in humans, notably 17 18 
1 reversible, side-effects like blushing/flushing, itching, gastrointestinal irritation,... -, notably with

2 slow/sustained-release niacin as compared to immediate-release niacin (Dalton and Berry, 1992;

3 Etchason et al., 1991; Lawrence, 1993; Pardue, 1961; Rader et al., 1992; Reimund and Ramos,

4 1994; Schwenk and Fisher, 1994; Stern, 2007); but the co-administration of betaine (Mccarty, 2000)

5 or methionine (Aronov et al., 1999) decreased hepatotoxic risk. Others reported the beneficial use

6 of myo-inositol hexanicotinate instead of niacin alone, myo-inositol hexanicotinate being free from

7 side effects (Welsh and Ede, 1961); and Baggenstos et al. (1967), via liver biopsies in 8 hypercholesterolemic humans chronically administered 1.5 to $6 \mathrm{~g}$ nicotinic acid, observed minor

9 histological alterations that were also reported in healthy patients, and concluded that the use of 10 nicotinic acid is not contraindicated in carefully supervised patients. Similarly, after one year of 11 nicotinic acid therapy in 17 patients, no significant hepatic alteration was found via the use of 12 several liver tests, and needle biopsies did not show any fatty changes or abnormalities (Parsons and 13 Flinn, 1959) although significant alterations in hepatic function tests were reported in another 14 studies 2 years later in 10 hypercholesterolemic patients among 36 (Parsons, 1961a). Recently, 15 lower doses of niacin up to $50.1 \mathrm{mg}$ daily have been tested in healthy volunteers and it has been 16 observed that a $16.7 \mathrm{mg}$-dose niacin does not cause flushing symptoms, that are sporadic at a 50.1 17 mg-dose (Schweikart et al., 2009). In addition, no change occurs concerning blood pressure, pulse 18 and skin temperature (Schweikart et al., 2009). In addition, niacin may reduce the release of FFA in 19 plasma through inhibition of catecholamine stimulation of TG lipolysis in adipose tissue (Arner, 20 1999), as notably shown in vitro (Carlson, 1963), leading to reduction of hepatic VLDL-TG 21 production (Chapman et al., 2010; Figge et al., 1988) and resulting in decreased plasma VLDL-TG 22 concentrations (Grundy et al., 1981). This may occur via either a reduced transport of FFA to the 23 liver or a direct inhibition of hepatic secretion/synthesis of ApoB-containing lipoproteins (Tato et 24 al., 1998). Others have shown in nondiabetic patients that the administration of $2 \mathrm{~g}$ daily of 25 nicotinic acid during 2 weeks reduces cholesterol synthesis by around 50\% (Nunn et al., 1961). And 26 a study in hyperlipidemic subjects that were administered 1 to $2 \mathrm{~g}$ daily nicotinic acid has lead to 
1 suggest that serum cholesterol reduction has to be attributed to reduction of cholesterol synthesis at 2 the hepatic level (Parsons, 1961a).

3 Compared to other lipotropes, physiological mechanisms involved in the lipotropic effect of 4 niacin therefore appear multifactorial as we have tentatively summarized and illustrated it in Figure $5 \quad 2 \mathrm{C}$ based on references cited previously and on those from Supplemental Table 1.

$7 \quad$ Pantothenic acid (vitamin B5)

8 As for niacin, apparent contradictory results have been also reported for pantothenic acid (Carter 9 and Hockaday, 1962; Griffith and Mulford, 1941b; Morgan and Lewis, 1953; Schaefer et al., 1942). 10 First, is was found that feeding rats with a B vitamin- (including thiamine, riboflavin, pantothenic 11 acid and pyridoxine) or a pantothenic acid-deficient diet prevented the development of fatty liver 12 (Engel, 1942; Morgan and Lewis, 1953), notably an increased cholesterol content in high13 cholesterol fed rats (Guehring et al., 1952), pantothenic acid being indirectly involved in the 14 transformation of acetate into cholesterol (Bloch and Rittenberg, 1942) via acetyl-CoA action and 15 being constitutive of the coenzyme. A 2-fold decreased food intake has been notably proposed as an 16 explanation for the observed reduced liver fat content of pantothenic acid-deficient rats 17 (Guggenheim and Olson, 1952). Others suggested that adrenal hormone production is reduced and 18 fat metabolism seriously impaired in pantothenic acid-deficient rats, adrenal hormone being 19 synthetized from cholesterol and pantothenic acid being involved in cholesterol synthesis (Morgan 20 and Lewis, 1953). However, fatty liver was reduced to normal level in rats when adding adequate 21 amounts of both inositol and choline to diets containing B vitamins thus moderating and relativising 22 the role that pantothenic acid may play in fatty liver development (Engel, 1942). The same year, it 23 was shown that pantothenic acid deficiency may lead to fatty liver in dogs (Schaefer et al., 1942) 24 and progressive increase in lipid globules in rat liver (Wirtschafter and Walsh, 1962). It was also 25 observed in pantothenic acid-deficient and high-fat fed rats a reduced hepatic neutral fat content 26 compared to pantothenic acid-supplemented and high-fat fed rats, with no difference for hepatic 
1 total cholesterol, free cholesterol and PL contents (Carter and Hockaday, 1962). The same

2 tendencies were reported with low-fat diets (Carter and Hockaday, 1962). Conversely, in the fifties,

3 Italian research teams reported lipotropic action of pantothenic acid in rats (Catolla Cavalcanti and

4 Levis, 1950,Turchetto et al., 1955). In pantothenic acid-deficient cats (only 0 to $3 \mathrm{mg} / \mathrm{kg}$ diet), some

5 hepatic fatty metamorphosis and fine and coarse vacuolar formation with lipids evenly deposited

6 were reported, no histological changes being observed when increasing pantothenic acid content of

7 the diet from 5 to $20 \mathrm{mg}$ despite a largely higher weight gain (Gershoff and Gottlieb, 1964). In

8 1968, Williams et al. showed that supplementing low-fat or high-fat fed rats with pantothenic acid

9 increased liver weight and FA contents but not that of PL with variations according to the FA

10 considered, e.g. higher levels of stearate and arachidonate in PL and higher proportion of linoleate

11 in TG (Williams et al., 1968). In 1969, Osumi et al. showed in rats that Ca-pantothenate partly

12 reduced the high hepatic TG content initially developed through a low-protein diet (Osumi et al.,

13 1969) while no change in hepatic lipid content was observed with pantothenic-deficient- vs normal-

14 fed rats (Fidanza et al., 1970). Latter, pantothenic acid carence has been shown to increase, but not

15 significantly, the total lipid content of liver in ducklings (Saheb and Demers, 1972). In pantothenic 16 acid-deficient rats kept on diet for more than 75 days, significantly lower phosphatidylcholine 17 content of $40 \%$ compared to non-deficient rats was also observed (Mahboob, 1975). In mice with 18 hypothalamic obesity induced by aurothioglucose, pantothenic acid derivatives 19 (phosphopantothenate, pantethine and panthenol) importantly and significantly reduced hepatic TG 20 content with no effect on total PL and free cholesterol, and significant effect upon total cholesterol 21 and cholesterol ester reduction by panthenol, reduced resistance to insulin and lipolysis activation 22 being hypothesized as possible mechanisms (Naruta and Buko, 2001).

More generally, pantothenic acid is recognized as maintaining normal hepatic functions

24 (Ueshima et al., 1956,Ueshima et al., 1958), and pantothenic acid deficiency lead to lower weight 
2 acid active form)(Kaplan and Lipmann, 1948; Lipmann et al., 1947; Novelli et al., 1949; Smith and 3 Song, 1996) that is active in $\beta$-oxidation, the main pathway to FA degradation (Figure 2B).

4 Accordingly, an increased in pantothenic acid consumption (5 mg daily) was shown to enhance

5 CoA activity in rat liver for the first 2 days compared to a control group (Causi et al., 1958). And

6 the hepatic CoA content (total, acid-soluble and long-chain acyl) was increased following 7 pantothenic acid supplementation for both low- and high-fat diets in rats while the CoA values were 8 always lower with the high-fat diet (18\%) than with the low-fat diet (6\%) (Williams et al., 1968).

9 The liver acyl-CoA content was otherwise increased by Ca-pantothenate after being decreased by a 10 low-protein diet (Osumi et al., 1969). Similarly to these results, it has been shown that the hepatic 11 total CoA content was significantly reduced in pantothenic acid-deficient weanling rats (Moiseenok 12 et al., 1987). Latter, the hepatic free CoA content reduction of developping mice treated with 13 valproate - that inhibits FA oxidation - was shown to be partly reversed when supplemented with 14 pantothenate plus L-carnitine and L-cysteine with no effect when L-carnitine was administered 15 alone, the increase in CoA content being also observed in absence of valproate (Thurston and 16 Hauhart, 1992). In addition, pantothenic acid-deficient rats exhibited a lower level of hepatic 17 peroxisomal $\beta$-oxidation that was restaured to normal level following supplementation: this down18 regulation of peroxisomal $\beta$-oxidation was paralleled with a reduced activity of the hepatic long19 chain acyl-CoA synthetase that activates FA degradation (Youssef et al., 1994). Authors suggested 20 that such an effect may result from an "adaptation to the reduced ability of the liver to activate FA 21 to their acyl-CoA thioesters" (Youssef et al., 1994).

Such results emphasized different pantothenic acid effects on hepatic lipid metabolism (see Supplemental Table 1 that reports most relevant studies). We believe that the contradictarory results obtained with both niacin and pantothenic acid probably depends on the presence or not of the other main lipotropes - choline, betaine, methionine and myo-inositol - or other B vitamins, but also on 
1 doses and animal species used, and on experimental scheme. In other words, the lipotropic action of

2 B-vitamins, notably niacin and pantothenic acid - probably exerts in synergy with other lipotropes.

3 This is the reason why in the end we have considered that niacin and pantothenic acid may be

4 considered as contributing to the overall lipotropic effect of PBF in normal dietary conditions, i.e. at

5 normal doses and including the presence of other lipotropes. Nowadays, it is otherwise commonly

6 used in lipotropic supplements.

$8 \quad$ Magnesium

9 Concerning magnesium, its depletion has been associated with cirrhosis (Koivisto et al., 2002), and 10 hypomagnesemia associated with NAFLD and non-alcoholic steatohepatitis (Hanje et al., 2006). A 11 low plasma level of magnesium has also been associated with insulin resistance (Rosolova et al., 12 1997), and a low magnesium diet was otherwise shown to decrease insulin sensitivity (Nadler et al., 13 1993). Magnesium has been also shown to reduce hyperlipidemia (Kisters et al., 1993).

14 More specifically, magnesium is well known as antioxidant (Freedman et al., 1992). It is 15 also particularly involved in the reaction of CoA with ATP (Mg-ATP complex) and FFA to yield 16 acyl-CoA (Figure 2B), and it activates CoA synthesis from pantothenic acid proportionally to the 17 presence of ATP. It is also required by mitochondria for oxidative phosphorylations that produce 18 ATP. All of these properties of magnesium play a role in the overall FA $\beta$-oxidation process (Figure 19 2B) (Andrieux-Domont and Le Van, 1970; Berg, 1959; Garfinkel and Garfinkel, 1985; Ingraham 20 and Green, 1958). The role of magnesium on FA oxidation was well illustrated by the dramatic 21 increase of palmitate oxidation reached in heart muscle mitochondria when increasing magnesium 22 concentration from 0.01 to $5 \mathrm{mM}$ in presence of carnitine $(\approx+800 \%)$ or acetylcarnitine $(\approx+950 \%$; 23 Supplemental Table 1) (Fritz, 1959).

As regards with these specific properties of magnesium and since increased oxidative stress 25 and insulin resistance may be associated with fatty liver, magnesium may be considered as 26 contributing to the overall lipotropic effect of PBF. It has moreover been cited as lipotrope in the 
1 clinical report of Colson and Gallay (Colson and Gallay, 1964) and is commonly used as such in 2 current commercial lipotrope complexes. There are however no human studies investigating the 3 effects of a magnesium therapy in patients with fatty liver.

4

5

6

7 5

\section{Other phytochemicals and plant extracts}

Lipotropic effect of choline, betaine, methionine and myo-inositol has been unravelled in rats quite early between 1932 and 1941 (Best and Huntsman, 1932; Gavin and Mchenry, 1941b; Tucker and Eckstein, 1937); then, always in rats, the lipotropic potential of vitamins B was apparently first emphasized around 1950 (Catolla Cavalcanti and Levis, 1950; Kelley et al., 1950; Tyner et al., 1950). The effect of carnitine on FA oxidation was reported in rat liver slice in 1959 (Fritz, 1959)

13 and carnitine was shown to importantly reduce hepatic TG content in choline-methionine-deficient and high-fat (30\%) fed rats (see Supplemental Tables 1 and 2) (Khairallah and Wolf, 1965).

From the survey and analysis of studies dealing with effect of plant compounds on hepatic lipid metabolism, it appears that this is not before the end of the sixties that research focused on other phytochemicals, notably hydroxycitric acid (HCA), organosulfur compounds, fiber, polyphenols, saponins, unsaturated and short-chain FA or melatonin (Supplemental Tables 2, 3 and 4). The exception was $\beta$-sitosterol that was reported in 1955 to reduce hepatic cholesterol content in high-cholesterol fed mice (Beher and Anthony, 1955). Around 1970, HCA was shown to decrease rate of lipogenesis and FA synthesis in rat liver (Lowenstein, 1971; Sullivan et al., 1972); and in the seventies, great interest was brought to fiber and derived compounds (Supplemental Table 3). Interest for the effect of polyphenols and derived compounds on hepatic lipid metabolism really begins in the nineties. Finally, concerning unsaturated FA, organosulfur compounds, short-chain FA and melatonin, their positive effect on hepatic lipid metabolism appear to have been put forward around respectively 1965, 1970, 1990 and 1995 (Supplemental Table 2). 
1 Now, we therefore considere all phytochemicals - other than betaine, choline, methionine,

2 myo-inositol, vitamins B, magnesium, carnitine and phytate - for which at least one significant

3 positive effect on lipid metabolism has been reported, be on total lipid, TG or cholesterol contents,

4 on lipogenic enzyme activities, FA oxidation enzyme activities, gene expression of PPAR $\alpha$ and

5 SREBP, or rate of lipogenesis (Supplemental Tables 1-4). However, in the following section will be

6 considered as lipotropic compounds sensu stricto only those that significantly reduce hepatic total

7 lipid or TG contents. Those decreasing only hepatic cholesterol content may not be considered as

8 lipotrope since steatosis is mostly concerned by TG accumulation or retention within hepatocytes

9 (Adams et al., 2005).

11 Specific plant compounds: hydroxycitric acid and organosulfur compounds

12 Besides the 8 previously defined lipotropes that are betaine, choline, myo-inositol, methionine, 13 magnesium, niacin, pantothenic acid and folates and that are quite ubiquitous in plants, other 14 phytochemicals that come from specific botanical families have been cited as having positive 15 effects on hepatic lipid metabolism: they were HCA (Lowenstein, 1971; Sullivan et al., 1972) 16 mainly isolated from fruits of the Garcinia family, notably Garcinia cambogia (Heymsfield et al., 17 1998; Lewis and Neelakantan, 1965) and used in commercial nutritional supplements that aim at 18 loosing weight, and cysteine-containing compounds as the organosulfured compounds found in 19 Allium species (e.g. s-ethyl cysteine and s-methyl cysteine in onion or garlic) ( Supplemental Table $202)$

\section{Hydroxycitric acid}

23 The lipotropic effect of HCA may however appear controversial as illustrated by the apparent 24 contradictory results obtained, as the increased post-prandial hepatic lipid content of chronically 25 high-fructose fed rat supplemented with HCA (Brandt et al., 2006), the decreased rate of 26 lipogenesis in rat liver following either i.v./i.p. HCA injection or orally ingested HCA (Lowenstein, 
1 1971,Sullivan et al., 1974b,Sullivan et al., 1972), the absence of effect on liver lipid content 2 following HCA supplementation in normal rats (Sullivan et al., 1974a) or in rats with 3 experimentally induced obesity (Sullivan and Triscari, 1977), the important increase in hepatic post4 prandial lipid content $(\approx+67 \%)$ in high-fructose fed rats (Brandt et al., 2006) or the significant 5 reduction of hepatic FA synthesis rate by HCA in high-fructose and high-glucose fed rats (Sullivan 6 et al., 1974b; Sullivan et al., 1977) (Table 2 and Supplemental Table 2). In addition, HCA was 7 shown in vitro to inhibit ATPCL/CCE (ATP-citrate lyase/citrate cleavage enzyme) activity, the 8 enzyme that catalyzes the split of citrate to oxaloacetate and acetyl CoA, the construction material 9 for FA: this inhibition of the conversion of carbohydrate metabolites into fat favours glycogen 10 accumulation within muscles and liver (Supplemental Table 2) (Watson et al., 1969).

Conversely, HCA was convincingly shown to significantly reduce weight gain or regain in rats (Brandt et al., 2006; Greenwood et al., 1981; Kang et al., 2007; Leonhardt and Langhans, 2002;

13 Nageswara Rao and Sakariah, 1988; Shara et al., 2004; Shara et al., 2003). This effect might be 14 notably attributed to the anorectic property of HCA in relation with an increased FA $\beta$-oxidation 15 (Leonhardt and Langhans, 2002) that would result from reduction in malonyl CoA production (via 16 inhibition of ATPCL) (McCarty, 1994), an inhibitor of CPT-1 (Figure 1b), and to the role that plays 17 FA oxidation in the metabolic control of food intake at high fat dose (Scharrer and Langhans, 18 1986). subjects after a 8 week-HCA treatment (750 mg daily, $\approx-4.5 \mathrm{~kg})($ Badmaev et al., 2002), while no 
1 (Heymsfield et al., 1998). In addition, HCA supplementation does not increase satiety in humans

2 (Kovacs et al., 2001a; Kovacs et al., 2001b) but may decrease blood levels in TG, LDL and 3 cholesterol (Badmaev et al., 2002; Preuss et al., 2004b). The effect on body weight loss might be in

4 relation with an increased short-term rate of fat oxidation as demonstrated in either athletes (Lim et 5 al., 2002) or untrained men (Tomita et al., 2003) although others have reported no significant effect 6 in sedentary adults at rest or during moderately intense exercise (Kriketos et al., 1999); and no 7 significant increased total fat oxidation was registered in enduranced-trained humans that were 8 given HCA solution of $19 \mathrm{~g} / \mathrm{L}$ at a level of $3.1 \mathrm{~mL} / \mathrm{kg}$ b.w. before and after exercise (Van Loon et 9 al., 2000). In mice, while a single HCA treatment of 10-30 $\mathrm{mg}$ had no effect on respiratory 10 exchange ratio, chronic HCA administration (10 mg HCA twice a day for 25 days) promote lipid 11 oxidation, either at rest or upon exercising conditions (Ishihara et al., 2000).

12 The lack of effect or the increase post-prandial content of hepatic lipid contents following 13 HCA supplementation in rats appears contradictory to the ability of HCA to importantly inhibit 14 hepatic rate of lipogenesis in chronically fed rats. This means that if, in vivo, HCA really inhibits 15 CCE activity, this does not reflect in lower total lipid content upon a long period of time. However, 16 to our knowledge, no study has investigated the specific effect of HCA on hepatic TG content. 17 Further studies are therefore needed before concluding or not HCA is a lipotrope sensu stricto.

19 Cysteine-containing compounds

20 Concerning water-soluble (e.g. s-allyl cysteine, s-ethyl cysteine, $n$-acetyl cysteine, s-propyl 21 cysteine) and lipid-soluble (e.g. diallyl sulphide and dipropyl sulphide) organosulfur compounds, 22 they have been shown in mice or rats fed a methionine-choline deficient (Lin et al., 2008), high-fat 23 (Lin and Yin, 2008) or high-cholesterol (Kumari and Augusti, 2007) diet to alleviate and/or to 24 protect liver from induced hepatotoxicity and from high saturated fat-associated oxidative damages, 25 but also to reduce hepatic biosynthesis of TG and cholesterol (Supplemental Table 2) (Kumari and 26 Augusti, 2007; Lin et al., 2004). Similar results were reported in diabetic mice (Hsu et al., 2004). 
1 Some of the mechanisms involved - notably as unravelled by using rat hepatocytes - are probably in

2 relation with a decreased activity of two lipogenic enzymes that are ME and FAS, a decreased

3 activity of $\mathrm{HMG}-\mathrm{CoA}$ reductase and a reduced rate of acetate or mevalonate incorporation into

4 lipids (Supplemental Table 2) (Gebhardt and Beck, 1996; Kumari and Augusti, 2007; Kumari et al.,

5 1995; Lin et al., 2008; Lin and Yin, 2008; Lin et al., 2004; Liu and Yeh, 2000; Yeh and Yeh, 1994).

6 This has been linked to significant depressed mRNA expressions for ME, FAS, HMG-CoA

7 reductase and SREBP-2 (Supplemental Table 2) (Lin and Yin, 2008). In addition, studies lead in

8 HepG2 cells suggest that the concerted action of several organosulfur compounds would allow

9 reaching a higher inhibition of acetate incorporation into cholesterol as compared to isolated

10 organosulfur compounds (i.e. s-allyl or s-propyl cysteine) (Lee and Yeh, 2003) and that inhibition

11 of hepatic cholesterol synthesis would mainly result from water-soluble organosulfur compounds

12 not lipid-soluble compounds that may become toxic at high doses (i.e. 1-4 mM) (Yeh and Liu,

13 2001). One may therefore conclude that results convincingly support lipotropic effect of 14 organosulfur compounds.

\section{Unsaturated and short-chain fatty acids, melatonin and para-aminobenzoic acid}

17 Mono-unsaturated and poly-unsaturated fatty acids

18 Unsaturated FA are common to both PBF and ABF. Results from studies lead with unsaturated FA 19 and oils specific to animal products (e.g. fish) have been therefore also presented in Supplemental 20 Table 2 to allow comparisons.

(Capanni et al., 2006; Spadaro et al., 2008). Results clearly showed a significant decrease in the Most of studies were lead in rats or mice (Supplemental Table 2). The only human studies degree of steatosis with $24-30 \%$ of subjects having no more steatosis diagnosed (Capanni et al., 2006; Spadaro et al., 2008). However, PUFA were either of animal origin (Capanni et al., 2006) or 26 no precision were given (Spadaro et al., 2008). Accordingly, n-3 PUFA have been recently 
1 proposed as a therapeutic liver drug to treat patients with NAFLD (Xin et al., 2008). It has been 2 otherwise observed in liver of NAFLD patients a marked enhancement in long-chain PUFA $3 n-6 / n-3$ ratio, such a condition being likely to "favour lipid synthesis over oxidation and 4 secretion", thereby leading to steatosis (Araya et al., 2004).

Among mechanisms involved, PUFA are known to inhibit the expression of FAS (Moon et 6 al., 2002) as shown with conjugated linoleic acid (CLA) in high-fat-fed rats (Choi et al., 2007), with 7 dietary long-chain n-3 FA-containing krill oil in high-fat-fed mice (Tandy et al., 2009), with PUFA 8 from safflower oil in high-fructose/glucose fed rats (Toussant et al., 1981), with methyl esters of 9 polyunsaturated vs long-chain saturated FA given to rats fed fat-free diet for 7 days (Clarke et al., 10 1977) and with $\alpha$-linolenic acid (18:3 n-3)-rich diet in both wild type and PPAR $\alpha$-null (KO) mice 11 (Supplemental Table 2) (Morise et al., 2009). And several authors have described n-3 PUFA as 12 "negative regulator of hepatic lipogenesis" (Alwayn et al., 2005; Sekiya et al., 2003; Spadaro et al., 13 2008). PUFA were also shown to increase PPAR $\alpha$ mRNA expression (Choi et al., 2007; Morise et 14 al., 2009) and to decrease SREBP mRNA expression (Sekiya et al., 2003) or activity (Di Nunzio et 15 al., 2010), to inhibit activities of several lipogenic (TG and cholesterol) enzymes that are ACC, 16 G6PDH, HMG-CoA reductase and ME and to increase activities of FA oxidation enzymes that are 17 CPT and acyl-CoA oxidase (ACO) (Supplemental Table 2). Cellular and nuclear mechanisms by 18 which PUFA may favour peroxisomal and mitochondrial FA $\beta$-oxidation via PPAR up-regulation 19 and inhibit TG and FA synthesis via SREBP1 down-regulation have been described and reviewed 20 by Clarke (Clarke, 2001). However, concerning SREBP, results are not always consistent since 21 some studies reported no effect or increased expression of SREBP (Gotoh et al., 2009; Morise et al., 22 2009) but this may be explained by the specific strains of mice used in these studies, i.e. $d b / d b$ mice 23 (with hyperlipidemic, diabetic and obese symptoms) (Gotoh et al., 2009) and PPAR $\alpha$-null (KO) 24 mice (Morise et al., 2009). In addition to these mechanisms, it was shown in ethanol-fed rats that 25 DHA and AA prevent from fatty liver development, and that protection of some mitochondrial 26 enzymes (aldehyde dehydrogenase, ATP synthase, and 3-ketoacyl-CoA thiolase) from oxidation by 
1 PUFA might be involved (Song et al., 2008). And in rats submitted to hypercaloric and fat-free

2 parenteral nutrition, it has been suggested that a lack of PUFA may lead to impaired lipid transport

3 (i.e. impaired formation of lipoproteins that exports lipids outside liver) and enhanced lipogenesis

4 (Goheen et al., 1983,Keim and Mares-Perlman, 1984).

As shown recently in mice fed synthetic diet containing lard (low in PUFA and highly

6 unsaturated FA, HUFA), canola oil (high in PUFA, i.e. linoleic and linolenic acids) or a mixture of

7 menhaden and fish/fungal oils (high in HUFA, i.e. AA, EPA and DHA), it seems that HUFA from

8 animal origin (menhaden/fish/fungal oil) are more efficient in preventing from steatosis than PUFA

9 from plant origin (canola oil) although linoleic and linolenic acids are both precursors in vivo of

10 HUFA (Sealls et al., 2008). Yet, MUFA-rich olive oil was shown to be more efficient in reducing

11 degree of steatosis in methionine-choline-deficient rats than PUFA-rich fish oil; and while olive oil

12 consumption significantly reduced hepatic TG content by around $29 \%$, fish oil failed to 13 (Supplemental Table 5) (Hussein et al., 2007). Accordingly, the role of oleic acid in olive oil to 14 prevent steatosis in NAFLD patients has been latter discussed (Assy et al., 2009). Indeed, oleic acid 15 is able to decrease NF- $\kappa \mathrm{B}$ activation and LDL oxidation while increasing insulin resistance that in 16 the end lead to dow- and up-regulation of respectively SREBP and PPAR $\alpha$ and PPAR $\gamma$ and 17 increased hepatic FA oxidation (Assy et al., 2009). However, several other phytochemicals would 18 also contribute to the overall lipotropic effect of olive oil, such as phenolic compounds, squalene, 19 lignans and hydroxytyrosol, which prompted Assy et al. to suggest that olive oil and, more 20 generally MUFA-rich foods, is a main contributor of the beneficial effect of the Mediterranean diet 21 in the primary prevention of NAFLD (Assy et al., 2009). Besides olive oil, PUFA/n-3 rich/low22 trans structured fat synthesized from flaxseed oil, butter fat and palm stearin was alo shown to exert 23 significant lipotropic effects, among which a decreased hepatic TG content of $16 \%$, an increased $\beta$ 24 oxidation of $96 \%$ and an increased $\mathrm{CPT}$ activity of $88 \%$ in $\mathrm{ApoE}^{-/-}$mice compared to ApoE $\mathrm{E}^{-/-}$mice 25 fed a 10\%-fat (commercial shortening, 53.4\% trans FA) diet (Supplemental Table 5) (Cho et al., 26 2009). However, results do not appear always consistent. Thus, the respective efficacity of different 
1 oils in improving various markers of hepatic lipid metabolism has been tested in rats fed initially a $2 \quad 10 \%$-fat diet rich in saturated lipids: while sunflower (n-6 PUFA-rich), linseed (enriched with $\alpha$ 3 linolenic acid) or sardine (n-3 PUFA-rich) oils importantly decreased TG content, and ACC and 4 G6PDH activities, olive oil (oleic acid-rich) failed to (Supplemental Table 5) (Takeuchi et al., 5 2001). However, all oils importantly and significantly increased $\beta$-oxidation and CPT activity (at 6 least $+100 \%$ ), olive oil remaining the less efficient (Takeuchi et al., 2001). These results appear 7 somewhat contradictory with those of Hussein et al. reported above with olive and fish oils 8 (Hussein et al., 2007). Discrepencies may be ascribed to the different models tested, i.e. methionine9 choline deficient vs $10 \%$-fat fed rats.

As for fiber and polyphenols, unsaturated FA are composed of numerous compounds and it 11 is difficult to test each one as regards with hepatic steatosis improvement. However, results tends to 12 show a lipotropic effect of unsaturated FA, with notably important TG reductions of $-83 \%$ with 13 arachidonic acid in ethanol fed rats (Goheen et al., 1983) and of around $-49 \%$ with linseed oil (rich 14 in $\alpha$-linolenic acid) in PPAR $\alpha$-null (KO) female mice fed high-fat diet (Morise et al., 2009) 15 (Supplemental Table 2). Although FA are not from natural origin, important reduction in lipogenic 16 enzyme activities were also reported with ethyl linoleate and methyl linolenate/linoleate/oleate 17 (Clarke et al., 1977; Toussant et al., 1981). In addition, decreased SREBP and increased PPAR were 18 also observed, which is also supportive and indicative of a decreased lipogenic activity 19 (Supplemental Table 2).

In the end, one may first wonder whether all unsaturated FA of plant origin are lipotropic or 21 not: if results appear still insufficient to definitively conclude, those reported in Supplemental Table 222 in both animals and humans tend to support a lipotropic effect whose significance vary according 23 to models and FA chosen. Secondly, one may wonder whether n-6 (e.g. arachidonic acid, C20:4 n24 6) and n-3 (e.g. $\alpha$-linolenic acid, C18:3 n-3) would have the same lipotropic potential. In humans, 25 only n-3 PUFA have been proposed to treat patients with NAFLD (Xin et al., 2008), excess n-6 26 consumption being pro-inflammatory (Lee et al., 2007a) and being likely to be involved in the 
1 promotion of hepatic necro-inflammation (Cortez-Pinto et al., 2006) that may transform NAFLD

2 into non-alcoholic steatohepatitis.

3

$4 \quad \underline{\text { Short-chain fatty acids }}$

5 Short-chain fatty acids (SCFA) mainly result in humans and animals from fiber fermentation and

6 the most important are acetate, propionate and butyrate. As for the previously PBF compounds, they

7 have been shown, either as isolated compound or in mixture, to exert positive and significant effects

8 on hepatic lipid metabolism (Supplemental Table 2). But only one study reported a significant

9 decrease in hepatic TG content (around 16\%) with acetic acid in high-fat fed mice (Kondo et al.,

10 2009). Among mechanisms involved, up-regulation of $\operatorname{PPAR} \alpha, \mathrm{ACO}$ and CPT-1, and down-

11 regulation of FAS gene expression were demonstrated (Kondo et al., 2009). Consequently, SCFA

12 being produced via fiber fermentation within colon, fiber may be considered as possibly indirectly 13 playing a role in these mechanisms.

14 Other studies mainly reported the inhibition effect of SCFA upon rate of cholesterol 15 synthesis as shown in isolated hepatocytes with propionic acid (Wright et al., 1990) or in liver slices 16 with SCFA mixture of acetic, propionic and butyric acids (Hara et al., 1999) (Supplemental Table 17 2). And hepatic acetate and propionate concentrations were shown to be negatively correlated with 18 hepatic cholesterol content in rats (Koseki et al., 1991).

20 Melatonin

21 In human, melatonin is synthesized from serotonin in pineal gland and is before all known as being 22 the central hormone that regulates chronobiological rhythms, notably sleeping. In plants, melatonin 23 is a strong antioxidant and also plays a role in its growth. To our knowledge, there is no database 24 for the melatonin content of PBF, and melatonin content of some PBF still remains unknown. 25 However, hazelnuts and walnuts are considered as good vegetable sources of melatonin; and 
1 melatonin is also found in algae, ginger, grape, cocoa, cereals (e.g. maize, rice and wheat), 2 tomatoes, potatoes and green vegetables.

Several studies have reported a protective effect of melatonin against liver injury in relation

4 with its antoxidant property and its effect on gene expression in relation with antioxidant status

5 (Catala et al., 2007; Leon et al., 2004; Sener et al., 2004; Subramanian et al., 2007; Taysi et al., 6 2003). More specifically, altough studies are scarce, melatonin has been reported in rats, mice and

7 minks to importantly reduce hepatic TG contents and to improve grade for steatosis (Supplemental 8 Table 2) (Kuzu et al., 2007; Nieminen et al., 2001; Pan et al., 2006; Sener et al., 2004; Shieh et al., 9 2009; Subramanian et al., 2007). However, doses used in rat and mice studies were high and un10 physiological (i.e. from $0.5-10 \mathrm{mg} / \mathrm{kg}$ b.w. injected i.p. and $10 \mathrm{mg} / \mathrm{L}$ of drinking water) (Pan et al., 11 2006; Sener et al., 2004). The study lead in minks used more physiological doses around $10 \mu \mathrm{g}$ 12 daily (Nieminen et al., 2001).

Mechanims involved in this lipotrope effect might notably include a reduced oxidative stress 14 - increased oxidative stress and lipid peroxidation being associated with steatosis - and decreased 15 insulin resistance (Kuzu et al., 2007; Sener et al., 2004). Increased insuline resistance is an 16 important parameter in the ethiology of fatty liver. Indeed, such decreased insulin sensitivity may 17 accelerate TG hydrolysis within adipose tissues releasing FFA within bloodstream, this latter being 18 then uptook in great amount by the liver and re-synthesized in TG forming excess fat deposits. 19 Deficiency in MTP and decreased synthesis of ApoB that are involved in VLDL assembly to export 
1 lipotropic effect. Indeed, PABA has been shown to decrease serum cholesterol level in men (Failey 2 and Childress, 1962), to play a role in folate formation (Barbieri et al., 1995), notably as 3 intermediate in the bacterial synthesis of folates (Wegkamp et al., 2007) and has been recognized as 4 stimulating bacteria growth (Briggs and Daft, 1955; Pfiffner and Bird, 1956). In addition, bacteria 5 are found in human intestine, folates are lipotropes, and PABA omission in the diet of guinea pig 6 was shown to lead to folic acid deficiency (Woodruff et al., 1953). Yet, although used in 7 commercial lipotropic complexes, the lipotropic effect of PABA, notably a reduced hepatic TG or 8 lipid content, has never been demonstrated, neither in animals nor in humans. It has only been 9 shown in rats that steatosis was associated with an increased level of acetylation due to inhibition of 10 FA oxidation, this being reflected with increased level of acetylated PABA in rat urine (Van Hung, 11 1953).

13 Fiber-type and polyphenol-type compounds

14 Plant-based foods are also well-known sources of fiber (soluble and insoluble), oligosaccharides, 15 polyphenols and phenolic-derived compounds that cannot be found in ABF. All of these 16 compounds have been shown to positively affect lipid metabolism in both humans and animals 17 according to various mechanisms. However, they have never been cited as lipotropes.

19 Soluble and insoluble fiber

20 Both soluble (e.g. pectin from sugarbeet fiber) and insoluble fiber (e.g. cellulose and insoluble 21 hemicellulose from wheat bran) have been convincingly reported to reduce hepatic TG and/or total $22 \mathrm{lipid} /$ fat contents in rats fed various steatogen diets (Supplemental Table 3). For example, 85\% 23 hepatic TG content reduction has been reached by supplementing diet with $5 \%$ lignin in high24 cholesterol (1\%) fed rats (Story et al., 1981). However, in rats fed normal diet, lipotropic effect of 25 fiber would be less conclusive (Schneeman and Richter, 1993). 
Concerning cholesterol, apparent contradictory results - i.e. lower hepatic content together

2 with higher HMG-CoA reductase activity and higher rate of synthesis - were also reported (Thomas

3 et al., 1983). This may be attributed to an adaptation resulting from the higher release of cholesterol

4 and its precursors (i.e. bile acids) within intestine via hydrophobic binding to insoluble fiber or

5 trapping within soluble and viscous fiber. Consequently, the liver compensates losses in cholesterol

6 by increasing its synthesis and turnover through an enhanced HMG-CoA reductase activity and rate

7 of cholesterol synthesis (Figure 2D). Thus, Thomas et al. have notably shown on liver slices of rats

8 fed for 1 month a $11 \%$-fat diet supplemented with $30 \%$ of neutral detergent fiber from blackgram

9 that incorporation of $\left[\mathrm{U}-{ }^{14} \mathrm{C}\right]$ glucose or $\left[1,2-{ }^{14} \mathrm{C}\right] \mathrm{Na}$-acetate into cholesterol was increased by

10 respectively 80 and $258 \%$ (Thomas et al., 1983).

11 Physico-chemical properties of fiber have therefore to be considered to explain their hepatic

12 lipid-lowering effect. For exemple, fiber, especially hydrophobic lignin (including in the fiber 13 definition), have been early shown to adsorb and/or sequestrate bile acid conjugates via 14 hydrophobic bounds (Eastwood and Mowbray, 1976; Eastwood, 1975; Eastwood and Girdwood, 15 1968; Eastwood and Hamilton, 1968) thus potentially stimulating cholesterol efflux from liver. 16 Latter, Mongeau and Brassard evaluated the bile salt binding capacity of various cereal products 17 ranging from $16.2 \mu \mathrm{mol}$ glycocholate $/ 0.2 \mathrm{~g}$ of neutral detergent fiber (NDF) for wheat germ to 34.2 $18 \mu \mathrm{mol}$ glycocholate/0.2 g NDF for spoon-size shredded wheat (Mongeau and Brassard, 1982). studied. Thus, recently, it has been shown in mice fed a $10 \%$ husk diet that genes encoding for FA 21 oxidation and lipogenesis were respectively up- and down-regulated after 3 weeks but the inverse 22 was observed after 10 weeks suggesting a "regulatory mechanism to restore the lowered plasma 23 cholesterol and TG levels" (Chan and Heng, 2008). However, at the hepatic cellular level, it is 24 unlikely that fiber compounds act directly on gene and explanations have probably to be found in 25 fiber-associated compounds like polyphenols and their resulting conjugated and metabolized forms 
1 and/or fiber fermentation products that are SCFA, especially propionic acid, all of them being able

2 to reach liver and directly impact cellular metabolism and gene expression.

3

$4 \quad$ Oligosacharides

.

8

Oligosaccharides from PBF are considered as fiber-type compounds that are completely fermented within colon and that include oligofructoses and galactosides like verbascose, stachyose and raffinose; but, to our knowledge, hepatic lipid-lowering effect has been mainly reported for oligofructoses like fructans (e.g. inulin) in rats fed standard, high-sucrose or high-fructose diet and

9 in obese Zucker rats (Supplemental Table 3) (Busserolles et al., 2003; Daubioul et al., 2002;

10 Daubioul et al., 2000; Kok et al., 1996a; Kok et al., 1996b; Sugatani et al., 2006). The action of

11 inulin-type fructans on TG and cholesterol metabolism has been recently reviewed by Beylot

12 (Beylot, 2005). Among mechanisms involved, fructans have been notably shown to decrease gene

13 expression and/or resulting activities of lipogenic enzymes that are ME, FAS, ACC, ATPCL/CCE 14 and G6PDH (Figure 2D, Table 3 and Supplemental Table 3) (Aghelli et al., 1998; Delzenne and 15 Kok, 1999). Such data tend to explain that the reduction of TG-rich lipoproteins (i.e. VLDL) 16 secretion observed in rats would be in relation with a decreased hepatic lipogenesis (Delzenne and 17 Daubioul, 2000). Other mechanism possibly include the production of proprionate - through 18 colonic fermentation of fructans - that was shown to inhibit lipogenesis in rat hepatocytes in vitro 19 (Supplemental Table 2) (Demigné et al., 1995,Wright et al., 1990). Beylot otherwise suggests that 20 "hypotriglyceridaemic action of fructans results rather from a decrease in the hepatic TG synthesis 21 than from a higher clearance of TG-rich lipoproteins" (Beylot, 2005). In their review, Delzenne and 22 Daubioul also proposed that $1^{\circ}$ ) fructans, by affecting glycemic and insulinemic responses, 23 indirectly modulate TG levels, insulin participating in the regulation of TG synthesis; and/or that $242^{\circ}$ ) since oligofructose may increase GLP-1 caecal concentration in rats fed oligofructose (Kok et al., 1998) and since GLP-1 may increase insulin sensitivity, this hormone is likely to be a modulator of lipid metabolism as well (Delzenne and Daubioul, 2000). This last hypothesis is supported by a 
1 recent study showing in hyperinsulinaemic subjects fed $+20 \mathrm{~g} / \mathrm{d}$ of wheat fiber a significant increase

2 in plasma GLP-1 concentration upon 12 months (Freeland et al., 2010).

3

$4 \quad$ Polyphenols

Polyphenols

Resistant starch? Not really a phytochemical? Specific of processed PBF except banana. Depends on process conditions (difficult to select as lipotrope from RS databases)

- Shimotoyodome (2010): high-fat mice

- Han (2005): high-cholesterol fed rats (no effect on cholesterol content)

- Han (2003): cholesterol-free diet fed rats

- Shao (2002): cholesterol ( $0.2 \mathrm{~g} /$ day : environ $1 \%$ diet?) fed rats

- Lopez (2001): normal rats (TG decrease)

- Cheng and Lai (2000): high-cholesterol rats (effect on TG)

compounds), li - Fernandez (2000): hypercholesterolemic guinea pigs

- Levrat (1996): 0.4\%-cholesterol fed rats

7 positive effects

8 quite recent an

9 specific hepatic lipid metabolism, to our knowledge, no study has reported a lipotropic effect of

10 polyphenols in humans.

In animal models, hepatic lipid metabolism improvement has been observed for the 4 four

12 classes of polyphenols, especially flavonoids and lignans (Supplemental Table 4). However,

13 significant hepatic TG reductions were reported only for lignans, and in lesser extent for flavonoids 14 (Supplemental Table 4). From studies reviewed in Supplemental Table 4, one can observe that for 15 the few one that investigated effect of polyphenols in non-steatosis models (i.e. with standard diets), 16 no significant effect on hepatic cholesterol and TG contents were observed (Nakamura et al., 2001; 17 Nakamura and Tonogai, 2002). In addition, most of studies are concerned with flavonoids and 18 lignans, and secondarily with phenolic acid and stilbenes (only one study) (Supplemental Table 4).

Compared to flavonoids and lignans, the few studies lead with phenolic acids, mainly ferulic

20 acid, did not support a conving lipotropic effect (Supplemental Table 4). For exemple, gallic acid 21 was shown to have no effect on FAS activity in vitro (Wang et al., 2003). The most significant 22 effect was the inhibition of HMG-CoA reductase by ferulic acid in high-cholesterol fed rats (Kim et 23 al., 2003). In this study, ferulic acid was also shown to significantly reduce acyl-CoA:cholesterol 24 acyltransferase (that forms cholesteryl esters from cholesterol) activity (Kim et al., 2003). However, 25 this is not sufficient to considere phenolic acids as having a lipotropic effect. 
1 Concerning flavonoids and lignans, sesamin (a lignan) has been reported to be a potent

2 inducer of hepatic FA oxidation in 10-15\%-fat fed rat (Ashakumary et al., 1999; Ide et al., 2001),

3 and the flaxseed lignan secoisolariciresinol (SECO) was recently shown to dose-dependently reduce

4 hepatic lipid accumulation in high-cholesterol fed rats (Felmlee et al., 2009). Major green tea

5 polyphenols (e.g. (-)-epigallocatechin-3-gallate) may prevent fatty liver disease in high-fat fed mice

6 (Bose et al., 2008); and various types of flavonoïds have been shown to prevent liver steatosis

7 (Dulloo et al., 1999; Klaus et al., 2005; Rumpler et al., 2001; Sachan and Hongu, 2000;

8 Shimotoyodome et al., 2005; Venables et al., 2008). Mechanisms involved would be notably the

9 ability of polyphenols to down-regulate and up-regulate gene expression of respectively lipogenic

10 and FA oxidation enzymes, and their resulting activities, but also to increase PPAR $\alpha$ and decrease

11 SREBP gene expression (Figure 2D and Supplemental Table 2). Flavonoids were notably shown to

12 strongly inhibit in vitro FAS activity (Wang et al., 2003; Wang and Tian, 2001). In a recent review,

13 the modulation of lipid homeostasis by flavonoids within liver was described (Peluso, 2006).

14 Briefly, flavonoids, via phosphodiesterase inhibition (Ko et al., 2004; Nichols and Morimoto, 1999 ,

15 2000), would notably stimulate lipolysis products from TG and cholesteryl esters (Peluso, 2006).

16 Indeed, phosphodiesterase inhibition would favour increase of cyclic adenosine monophosphate

17 (cAMP) level, activation of proteine kinase A, subsequent increase in hepatic triacylglycerol

18 hydrolase activity and $\beta$-oxidation of lipidic hydrolysis products (Peluso, 2006).

19 Concerning the fourth class of polyphebnols that are stilbenes, despite rarity of studies, the

20 only one reported in Supplemental Table 4 brought interesting results for leading future studies.

21 Stilbenes (i.e. cajanin, and longistylin C and A) containing extract/fraction from Cajanus cajan

22 supplemented at a level of $200 \mathrm{mg} / \mathrm{kg}$ b.w. allowed significantly reducing TG and total cholesterol

23 contents in hypercholesterolemic mice by respectively 14 and 23\% (Luo et al., 2008).

24 However, polyphenols are a huge phytochemical family, composed of several hundreds of

25 different compounds with probable different effects on hepatic lipid metabolism: all have probably

26 not a lipotropic effect sensu stricto. In literature, polyphenol content of PBF is mostly expressed by 
1 the Total Phenolic Compound (TPC) content (estimated via the Folin Ciocalteu's colorimetric

2 method). The TPC content corresponds to the easily extractable fraction and obviously does not

3 include only one type of polyphenol. However, this is among this polyphenol fraction that are to be

4 found those the most likely to be absorbed within small intestine and, consequently, the most likely

5 to exert a potential lipotropic action. One may therefore consider TPC content as a whole compound

6 with a potential lipotropic effect.

Accordingly, rather than to focuse on an isolated compound, more and more studies now

8 investigate the effect of ethanol- and/or water-extractable polyphenols from plants on hepatic lipid

9 metabolism in various animal models (Supplemental Table 4). For example, sylimarin and green tea

10 polyphenol extracts significantly reduced degree of steatosis and hepatic TG contents in 11 respectively hamsters fed a 10\%-fat and 0.2\%-cholesterol diet (Lin et al., 2009) and in leptin12 deficient (ob/ob) mice (Bruno et al., 2008).

14 Curcumin

15 Curcumin is not classified as a polyphenol sensu stricto but may be considered as a polyphenol16 derived compound (Figure 1). Among the two studies reported in Supplemental Table 4, curcumin 17 was interestingly shown to significantly decrease hepatic TG content by $22 \%$ in high-cholesterol 18 fed rats (Seetharamaiah and Chandrasekhara, 1993).

\section{$20 \underline{\text { Saponins }}$}

21 As curcumin, saponins are not sensu stricto polyphenols but possess a polyphenol-like chemical 22 structure (Figure 1). They are generally included in the fiber fraction. Studies are less recent than 23 with polyphenols (Supplemental Table 4). Their consumption or injection may lead to reduced 24 hepatic fat deposits or lipid contents (TG and cholesterol) (Khanal et al., 2009; Onning and Asp, 25 1995). For example, plant saponins from Aralia mandshurica and commercial white saponins 26 reduced hepatic TG contents by respectively $-40 /-35 \%$ and $-39 /-20 \%$ in high-fat (Wojcicki et al., 
1 1977) and high-cholesterol (Oakenfull et al., 1979) fed rats (Supplemental Table 4). Paradoxically,

2 saponin supplementation also lead to an increased rate of liver cholesterol synthesis as shown with 3 ginsenosides purified from ginseng in rats (Supplemental Table 4) (Sakakibara et al., 1975). This 4 has probably to be attributed to the same effect as for fiber, i.e. the adsorption of bile salts by 5 saponins within digestive tract that thereafter stimulates cholesterol turnover and hepatic synthesis 6 (Figure 2D). Indeed, saponins are most of the time associated with fiber within food matrix.

$7 \quad$ Coumarin: Auraptene in fatty long Evans rats $\{$ Nagao, $2010 \# 22917\}$

8 Alkylresorcinols

9 Alkylresorcinols are mainly found in wheat and rye in a range of around $30-150 \mathrm{mg} / 100 \mathrm{~g}$ and also 10 exhibit a polyphenol-like chemical structure (Ross et al., 2004b). Although not demonstrated 11 directly in vivo, alkylresorcinols were shown in vitro to importantly inhibit GPDH activity, the key 12 enzyme in TG synthesis and to reduce TG accumulation within 3T3-L1 cells (Rejman and 13 Kozubek, 2003), suggesting that alkylresorcinols might exert in vivo a potential lipotropic effect. In 14 addition, they were reported to significantly decrease total hepatic cholesterol content in rats (Ross 15 et al., 2004a). But further studies are needed to test in animal models of fatty liver the effect of 16 alkylresorcinol on TG or total lipid content.

18 Cholesterol-lowering phytochemicals

19 Several phytochemicals are generally tested for their cholesterol-lowering properties, notably at the 20 plasma level. They are $\gamma$ oryzanol, tocotrienols, policosanol and phystosterols.

22 Gamma-oryzanol

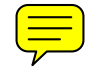

23 Gamma-oryzanol is a mixture of ferulic acid esters of triterpene alcohols and sterols (Figure 1) 24 extracted from rice bran oil. Among the four studies we reported in Supplemental Table 4, $\gamma$ 25 oryzanol was shown to reduce hepatic TG contents in high-cholesterol fed rats, but effect was 
1 Chandrasekhara, 1988, 1993). In the two other studies, $\gamma$-oryzanol was reported to signficantly

2 reduce hepatic cholesterol content by $19 \%$ but failed to significantly inhibit HMG-CoA reductase

3 activity in respectively hypercholesterolemic rats (Suh et al., 2005) and hamsters (Rong et al.,

4 1997). Further studies would be necessary to definitively conclude on the lipotrope status of $\gamma$ -

5 oryzanol.

$7 \quad$ Tocotrienols

8 Tocotrienols ( $\alpha, \beta, \gamma$ and $\delta$ ) are mainly found in whole-grain cereals (especially in wheat germ) and

9 unrefined vegetable oils, and belong to the vitamin $\mathrm{E}$ family together with tocopherols $(\alpha, \beta, \gamma$ and $10 \delta$ ). Tocotrienols are recognized as hypocholesterolemic compounds in both humans and animals 11 (Cicero and Gaddi, 2001; Minhajuddin et al., 2005; Qureshi et al., 1997). At the hepatic level, its 12 main reported effect on lipid metabolism is its ability to inhibit HMG-CoA reductase as shown in 13 cockerels (Qureshi et al., 1986) and guinea pigs (Khor et al., 1995), and to reduce subsequent rate of 14 cholesterol biosynthesis as shown in human HepG2 cells (Parker et al., 1993). More specifically, 15 both $\delta$ - and $\gamma$-tocotrienols have been shown in vitro to stimulate ubiquitination and degradation of 16 HMG-CoA reductase, and only $\delta$-tocotrienols has been shown to completely block SREBP-2 17 processing (Song and Debose-Boyd, 2006). In the end, $\gamma$-tocotrienol importantly increases LDL 18 receptor protein level in HepG2 cells (Parker et al., 1993). However, in the same time, FAS activity 19 was significantly increased by around $40 \%$ in cockerels upon tocotrienol supplementation at a $20 \quad 0.002 \%$ level (Supplemental Table 2) (Qureshi et al., 1986). Such results do not support a lipotropic 21 effect of tocotrieneols.

23 Policosanol

24 Policosanol is a mixture of high-molecular-mass aliphated alcohols initially isolated and purified 25 from sugar cane wax. It is mainly composed of octacosanol followed by triacontanol and 26 hexacosanol; other alcohols - tetracosanol, heptacosanol, nonacosanol, dodriacontanol and 
1 tetratriacontanol - are minor components. As tocotrienols, it is first recognized as a serum lipid-

2 lowering agent able to protect from cardiovascular diseases (Gouni-Berthold and Berthold, 2002;

3 McCarty, 2002; Varady et al., 2003). And, as tocotrienols, it may inhibit HMG-CoA reductase

4 activity (Mccarty, 2002) and increase LDL receptor protein level as shown via an increased hepatic

5 LDL-binding activity (Menendez et al., 1996,Menendez et al., 1997). Studies are scarce but it has

6 also been shown in hypercholeterolemic rabbits to significantly decrease hepatic cholesterol

7 synthesis (Menendez et al., 1997). Similar results were obtained in cultured human fibroblasts

8 (Menendez et al., 1994). But, to our knowledge, no effect on hepatic TG content has been reported

9 (Supplemental Table 2). As tocotrienols, policosanol cannot be therefore considered as having a 10 lipotropic effect sensu stricto.

12 Phytosterols

13 As early as 1956, it was shown that $\beta$-sitosterol (20 to $25 \mathrm{~g}$ daily) could reduce serum cholesterol in 14 patients with hypothyroidism by around $20 \%$ (Best and Duncan, 1956). But, to our knowledge, 15 there is no studies lead in humans to investigate the effect of phytosterol consumption on steatosis. 16 In animal models, phytosterols have no significant effects on hepatic TG content contrary to 17 cholesterol content (Supplemental Table 4). Yet, phytosterols were shown to increase HMG-CoA 18 reductase, CYP7A1 and sterol 27-hydroxylase activities: such enhanced activities may be explained 19 by the increased cholesterol release within small intestine under the action of phytosterols, which in 20 turn stimulates cholesterol synthesis to compensate such intestinal losses (Moghadasian et al., 21 2001), similarly to what occurs with fiber or saponins. Mechanisms underlying the cholesterol22 lowering properties of phytosterols have been otherwise thoroughly described by Brufau et al. 23 (Brufau et al., 2008). Besides, phytosterols were shown to importantly decrease hepatic ACC, ME 24 and G6PDH activities in high-cholesterol fed rats (Figure 2D) (Laraki et al., 1993). Although these 25 enzymes are directly involved in FA synthesis, we lack studies demonstrating a significant reduced 26 hepatic TG and/or lipid contents to conclude that phytosterols are lipotropic. 
2 Plant or plant-based food extracts

3 Plant-based foods may contain a whole set of lipotropes. Thus, the effect of foods or of their

4 extracts on steatosis is particularly relevant to study and closer to the nutritional reality than the

5 study of isolated compounds, often used at doses higher than that really consumed by humans.

Thus, some authors focused on various plant extracts rather than on a particular compound.

7 Studies are numerous and all could not have been cited in Supplemental Table 5. It is interesting to 8 note that complex foods or food extracts may lead to similar or enhanced lipotrope-like effects than

9 isolated compounds, i.e. mainly decreased hepatic TG and TC contents, increased activities of 10 enzymes involved in FA oxidation, decreased activities of enzymes involved in FA and cholesterol 11 synthesis. For exemple, plant extract from Platycodi radix was more efficient in reducing TG ($1244 \%$ ) than crude saponins (-17\%, NS) from the food extract (Supplemental Table 5) (Kwon et al., 13 2009b); and while tomato powder significantly reduced by $22 \%$ hepatic TG content in rats fed 14 standard diet, lycopene alone in the same amount than in tomato powder had no effect (Alshatwi et 15 al., 2010). Literature survey also unravels that foods tested cover a large range of PBF that are 16 cereal products, vegetable oils, fruits, seeds, vegetables, beverages or leaf extracts (Supplemental 17 Table 5).

However, the whole food package is not always more efficient towards liver steatosis or 19 associated lipid metabolism parameters than the isolated compound. For example, purified 20 polyphenols from Hibiscus sabdariffa (74\% content) had more marked effect on hepatic cholesterol 21 and TG contents than the corresponding plant-extract containing 2\% polyphenols (Yang et al., 22 2010). Some antinutrients from leaf extracts like tannins and saponins may be involved in impaired 23 hepatic functions as suggested by de Melo et al. who observed in rat liver higher levels of lipids and 24 cholesterol following cassava leaves flour consumption compared to control (De Melo et al., 2008). 25 Otherwise, it was shown with Ziziphus Mauritania leaf extract that pre-treatment (30 min before 26 alcohol administration) was more efficient than co- or post-administration in reducing hepatic 
1 cholesterol and TG contents of chronic alcohol administered rats (Dahiru and Obidoa, 2009).

2 Finally, the importance of interactions that exist between phytochemicals and micronutrients within 3 PBF is well illustrated by a study investigating the effect of rice bran, defatted rice bran and rice 4 bran oils with or without gum and wax on hepatic cholesterol and TG contents in 5 hypercholesterolemic hamsters (Kahlon et al., 1992). Results showed various ranges of TG and 6 cholesterol reductions according to bran fraction tested, e.g. ranking from $-14 \%$ hepatic TG content 7 (non significant) for defatted rice bran + rice bran oil to $-33 \%$ (significant) for whole rice bran 8 (Kahlon et al., 1992).

COMPARISON OF THE POTENTIAL LIPOTROPIC EFFECT OF THE 12 DIFFERENT CLASSES OF PLANT COMPOUNDS AS UNRAVELLED FROM

\section{RAT STUDIES}

\section{Study selection}

18 The lipotropic potential of each plant compound have been evaluated by selecting studies from 19 supplemental Tables 1-4. To allow relevant comparisons, only studies lead in rats fed steatogen 20 diet supplemented with phytochemicals have been considered. Selected steatogen diets are those 21 involving excess fat, sucrose, glucose and fructose percentages, alcohol and lipotrope deficiencies. 22 We therefore chose to select only steatogenic diets of nutritional origin; fatty liver provoked by 23 chemicals or drugs like $\mathrm{CCl}_{4}$ or DDT were not considered. Finally, 3 studies using obese fa/fa 24 Zucker rats were also selected since these rats developed fatty liver (Daubioul et al., 2002) and since many of its metabolic abnormalities, including leptin and insuline resistance and hyperlipidemia, are observed in human obesity (Kurtz et al., 1989; Marchesini et al., 1999; Sharabi 
1 and Eldad, 2000; Shimizu et al., 2007; Silverman et al., 1989). One study is concerned with HCA

2 (Sullivan et al., 1977) and two with oligofructose (Daubioul et al., 2002; Daubioul et al., 2000).

3 However, in order to obtain a sufficient number of data, all the durations for feeding periods and all

4 the percentages for phytochemical supplementation have been selected. Markers of lipid

5 metabolism chosen were those the most common to a maximum of phytochemicals, i.e. hepatic

6 total lipid/fat, TG and cholesterol contents, activity of main lipogenic enzymes (FAS, ME,

$7 \mathrm{G} 6 \mathrm{PDH}, \mathrm{ACC} / \mathrm{CBX}$ and $\mathrm{ATPCL} / \mathrm{CCE}$ ), and mRNA levels of 2 transcription factors that are

8 PPAR $\alpha$ and SREBP; PPAR $\alpha$ up-regulating peroxisome proliferation involved in FA $\beta$-oxidation

9 and SREBP up-regulating synthesis of enzymes involved in sterol biosynthesis. As a result, 4, 12,

$1010,7,3,2$ and 3 studies have been selected for respectively betaine, choline, myo-inositol, 11 methionine, niacin, pantothenic acid and folates; 8, 3, 2, 3 and 2 studies for respectively carnitine, 12 HCA, organosulfur compounds, MUFA/PUFA and melatonin; 14, 5 and 7 studies for respectively 13 soluble/insoluble fiber, phytic acid and oligosaccharides; 2, 4, 8, 2, 4, 4, 3 and 3 studies for 14 respectively phenolic acids, flavonoids, lignans, curcumin, saponins, phytosterols, $\gamma$-oryzanol and 15 polyphenol-rich plant extracts, i.e. a total of 115 studies which corresponds to around $30 \%$ of 16 studies reported in Supplemental Tables. The highest numbers of studies were therefore found in 17 the order fiber $>$ choline $>$ myo-inositol $>$ carnitine $=$ lignans. The collected data are synthesized 18 within Tables 2 and 3. Percentage changes for hepatic total lipids/fat, TG and cholesterol contents 19 are presented in Figure 3 A-C while percentage changes for lipogenic enzyme activity are 20 presented in Figures 4 A-E. Considering all compounds, feeding periods cover a range of 1 to 182 21 days while supplementation percentages cover a range from around $1 \mathrm{ppm}$ for folates to $30 \%$ for 22 fiber (Tables 2 and 3). 
2 First, concerning hepatic lipid contents, the most striking reductions, i.e. $>80 \%$, are reached for 3 total lipid and TG contents with choline, methionine, myo-inositol, fiber (lignin) and phytic acid. 4 Although only one study could have been selected, unsaturated FA (i.e. arachidonic acid in the 5 study concerned) may also lead to important reduction in total lipid/fat (-63\%) and TG contents ($683 \%$ ) (Goheen et al., 1983). Conversely, increases in hepatic lipid percentages ranged between $+1 \%$

7 for cholesterol content with phenolic acids and $+136 \%$ for TG content with lignans with significant 8 effects reached only for fiber and lignans on cholesterol content (resp. +17 and $+21 \%$ ), and lignans 9 on TG content $(+136 \%)$. (Table 3$)$.

If increased cholesterol contents are not unexpected with fiber since they may stimulate 11 hepatic cholesterol turnover consequently to an increase faecal excretion, that of TG content with 12 lignans is very surprising. However, the effect has been reported for fish oil only (at a level of $8 \%$ ) 13 not with palm and safflower oils (resp. -68 and $-23 \%$ TG content reduction, $p<0.05$ ) (Ide et al., 14 2004). As an explanation, authors suggested that the interaction of sesamin with fish oil may have 15 change expression of genes involved in VLDL assembly and production, impairing hepatic TG 16 excretion (Ide et al., 2004). Concerning other studies with lignans, TG content modifications were 17 all $\leq 0$ within the range $[\approx 0 /-68 \%]$ (Figure $3 \mathrm{~B}$ and Supplemental Table 4 ). It is interesting to note 18 that the sole increase was obtained with the only oil rich in HUFA (10\% of $20: 5 n-3$ and $32.6 \%$ of $1922: 6 n-3)$ that is fish oil, oils used in other studies being all vegetable oils (safflower, palm and 20 coconut oil) with largely less HUFA contents: indeed, palm oil is characterized by a high level of $2116: 0(\approx 45 \%)$ and 18:1n-9 $(\approx 39 \%)$ (Ide et al., 2004), safflower oil by a high level of 18:2n-6 $(\approx$ $2278 \%)$ and $18: 1 \mathrm{n}-9(\approx 13 \%)$ (Ide et al., 2004) and coconut oil by a high level of saturated FA $(\approx 87 \%)$ 23 (USDA, 2005e). Another explanation for the high increased TG content of $+136 \%$ might therefore 24 rely on the fact that fish oil is a n-3 PUFA-rich oil contrary to palm (saturated and MUFA-rich) and 25 safflower (n-6 PUFA-rich) oils. Indeed, PUFA are known to be lipotropic (see above) which may 26 have lead to the absence of TG reduction effect by sesamin: otherwise, in this study, palm and 
1 safflower oils alone lead to respectively 5.8- and 3.2-fold more hepatic TG accumulation than fish

2 oil for which level of hepatic TG is quite low (14 $\mu \mathrm{mol} / \mathrm{g}$ liver) (Ide et al., 2004). This means that 3 the $10 \%$-fish oil diet was not steatogen.

Besides, although no level of significance was given, a surprising $+47 \%$ increase in hepatic

5 TG content has been found in rats (Table 3) when increasing the neutral detergent fiber content 6 (from wheat bran) of the diet from 2.83 to $11.17 \%$ at the expense of the protein content (from 19.01 7 to 9.31\%) (Supplemental Table 3) (Stewart et al., 1987). One explanation may be found in that low8 protein diet may be steatogen (Best et al., 1955) and that normal protein levels recommended are 9 generally $20 \%$ of the diet for growing rats and $14 \%$ for adult rats (Reeves et al., 1993). In addition, 10 in the study by Stewart et al., at a constant fiber and fat levels of respectively 7 and $17.5 \%$, the 11 increase in protein level from 21.93 to $35.93 \%$ lead to $+82 \%$ TG content (Stewart et al., 1987). It 12 may be hypothesized that a too important distance from standard protein level remains steatogen 13 whatever the level of fiber.

14 Concerning hepatic cholesterol content reduction, it tends to be less important than TG 15 content reduction with choline, myo-inositol, carnitine, phytic acid and oligofructose, while 16 opposite tendency may be observed with phytosterols (Tables 2 and 3). Finally, maximal hepatic 17 cholesterol content reduction reached are quite high for choline $(-56 \%)$, folates $(-51 \%)$, carnitine ($1860 \%)$, fiber (-75\%), saponins (-52\%) and phytosterols (-76\%) (Tables 2 and 3$)$.

\section{Influence of phytochemicals on hepatic lipogenic enzyme activities following steatogen diet}

\section{2 consumption by rats}

24 Myo-inositol, unsaturated FA, phytic acid, oligofructose and lignans were the compounds the most 25 often tested for their ability to reduce lipogenic enzyme activities in rats, and results showed that 26 these compounds may be all efficient depressors of them (Figures 4 A-E). The most important 
1 reductions $(\geq 50 \%)$ are obtained with unsaturated FA and lignans on FAS, ME, G6PDH, $2 \mathrm{ACC} / \mathrm{CBX}$ and/or ATPCL/CCE activities (Tables 2 and 3; Figures 4 A-E). However, unsaturated 3 FA tested here were all either methylated or ethylated, and therefore they did not correspond to the 4 natural form found in PBF (Supplemental Table 2) (Clarke et al., 1977; Toussant et al., 1981). A $565 \%$-decrease has been also obtained with phytic acid on FAS (Figures 4A) activity (Katayama, 6 1997a). One unexpected result as regards with effect on other lipogenic enzymes is the tendency of 7 lignans to increase ME activity (up to $+125 \%$, Table 3 ). However, in the study reporting this result, 8 i.e. by Ashakumary et al., ME activity was first reduced by $50 \%$ at $0.1 \%$ sesamin level then 9 increased by 25 and $125 \%$ at respectively 0.2 and $0.5 \%$ level of the diet, and this was paralleled by 10 increasing mRNA levels for the enzyme (Ashakumary et al., 1999). These results were later 11 confirmed in rats with quite the same conditions (Supplemental Table 4) (Ide et al., 2001). One 12 explanation may be based on the PPAR-dependent regulation of ME gene expression unlike other 13 other lipogenic enzymes like FAS or G6PDH (Castelein et al., 1994). Thus, lipotropes, by inducing 14 increased PPAR mRNA expression may increase in the same time ME activity: this underlined the 15 dual role played by the transcription factor PPAR that both favour FA $\beta$-oxidation and ME activity 16 (Castelein et al., 1994).

\section{Influence of phytochemicals on hepatic PPAR and SREBP mRNA expression following} 20 steatogen diet consumption by rats

22 Concerning changes in the levels of both transcription factors PPAR $\alpha$ and SREBP, data collected 23 are scarce, but they indicate that flavonoids importantly increase PPAR $\alpha$ mRNA levels, and that 24 lignan importantly reduce that of SREBP, both results being in agreement with a lipotropic effect, 25 i.e. a reduction of hepatic lipid content (Tables 2 and 3). 


\section{THE WHOLE LIPOTROPE VS ANTIXODANT "PACKAGE"}

3

4 The antioxidant "package"

The lipotropic potential of PBF has quite interesting similarities with the concept of antioxidant capacity of PBF. Indeed, lipotropes and antioxidants both include several phytochemicals with different physiological modes of action dedicated to reach a same physiological effect: either a

9 decreased fatty liver or a decreased oxidative stress. Indeed, it is today more and more assumed that it is preferable to consume several antioxidants in a limited amount than only one at high dose (Murakami et al., 2003,Stanner et al., 2004), as the ATBC (Alpha-Tocopherol, Beta-Carotene Cancer) study has dramatically showed it, with a $8 \%$ increased mortality and $18 \%$ increase in lung cancer registered in the group of male smokers consuming a supplemented dose of $20 \mathrm{mg} / \mathrm{day} \beta$ carotene (The Alpha-Tocopherol, Beta Carotene Cancer Prevention Study Group, 1994). This underlines that high dose of only one substance may be pro-oxidative and harmful. As stated by Stanner et al., "The most prudent public health advice remains to increase the consumption of plant

17 foods, as such dietary patterns are associated with reduced risk of chronic diseases" (Stanner et al., 18 2004). The synergy between antioxidants appears therefore essential since one antioxidant may 19 regenerate the other after being oxidized. This is well illustrated by vitamin $\mathrm{C}$ that regenerates 20 oxidized vitamin $\mathrm{E}$ and glutathione that regenerates oxidized vitamin $\mathrm{C}$. This has also been 21 demonstrated with various combinations of antioxidants, e.g. green tea extract, quercetin and folic 22 acid protect better against $\mathrm{H}_{2} \mathrm{O}_{2}$-induced cellular damages than compound alone (Jeong et al., 23 2005), combinations of various antioxidants (i.e. ascorbic acid, caffeic acid, quercetin and urate) 24 have been shown in vitro to have a higher antioxidant potential than the sum of their components 25 (Parker et al., 2010), and tomato powder is more protective against elevated serum MDA levels in rats receiving $\mathrm{H}_{2} \mathrm{O}_{2}$ than isolated lycopene (Alshatwi et al., 2010). Thus, at least 30 phytochemicals 
1 or group of compounds in whole-grain cereals have been reported to have an antioxidant effect in 2 vivo, direct or indirect (Fardet, 2009); and their physiological mode of action may express very 3 differently by trapping reactive oxygen species (ROS), breaking oxidative chain reactions, 4 detoxifying potentially oxidative compounds, regulating glutathione synthesis or being co-factors 5 of enzymes involved in the antioxidant defense (Fardet et al., 2008). More generally, it has been 6 reviewed that optimal health - notably as regards with CVD and cancer prevention - requires the

7 combined actions of vitamins E, C and A, and of carotenoids and other "conutrients" contained in 8 fruits and vegetables (Gey, 1998).

\section{The lipotropic "package”}

15 mode of action towards lipid metabolism in liver that can complete between each others. The issue 16 of synergism for lipotropes might be well illustrated by the example of niacin that may be 17 hepatotoxic and produce other harmful side-effects (e.g. flushing and nausea) at high doses within 18 a therapeutic context (McKenney et al., 1994), but may be beneficial at lower dose and/or 19 accompanied with other lipotropes such as betaine (McCarty, 2000), choline (Wenru et al., 1994), 20 folates (Mccarty, 2000), methionine (Aronov et al., 1999) or myo-inositol in the form of myo21 inositol hexanicotinate (or hexanicite) that produces a sustained-release of nicotinic acid together 22 with absence of the side effects observed when niacin is administered alone (El-Enein et al., 1983; 23 Mercier et al., 1967; Welsh and Ede, 1961). There are several other examples of the lipotropic 24 effect in rats of one compound reinforced and improved by the addition of another lipotrope as it 25 was shown with pantothenic acid and myo-inositol (Catolla Cavalcanti and Levis, 1950), with 26 choline and folates (Laird et al., 1965), with choline and carnitine (Ball, 1964) and with choline 
1 and myo-inositol (Andersen and Holub, 1980; Engel, 1942; Kotaki et al., 1968). For exemple, in

2 the study of Kotaki et al., while the use of only choline or myo-inositol only partly cures fatty liver

3 in rats, the use of both compounds almost completely cured rats (Kotaki et al., 1968). Similarly, the

4 lipotropic effect in rats fed either a high-fat or a B vitamin-deficient diet has been shown to be at its

5 optimum when combining respectively the consumption of choline, folic acid, inositol and

6 cobalamine (Drill, 1954) and the consumption of B vitamins, choline and myo-inositol, the only

7 consumption of B vitamin in this latter study unexpectedly aggravating fatty liver (Shils and

8 Stewart, 1954). It was also shown in rats fed choline-deficient diet that $0.5 \%$-methionine

9 supplementation lead to increased total hepatic lipid content, probably as a result of dietary amino

10 acid imbalance (Arvidson and Asp, 1982). These examples illustrated well the interactions or the

11 interferences that may exist between lipotropes, some B vitamins being for exemple able to

12 potentiate and/or to catalize the lipotrope action of other lipotropes such as choline. Similarly to

13 niacin when used at clinical doses, some phenolic compounds that are antioxidant at low doses

14 may have pro-oxidative effect at higher doses as shown with quinones (menadione and

15 hydroxyquinone) in cultured HepG2 cells (Rushmore et al., 1991) and isolated rat hepatocytes

16 (Thor et al., 1982).

17 This raises the issue that a single agent at high dose may have physiological side-effects

18 that would be masked by combining several agents at lower doses with complementary 19 physiological mechanisms of action. Such an issue has been notably emphasized for the 20 carcinogenic process that involves several stages with different impaired physiological 21 mechanisms and that might be best prevented by combining multiple agents with distinct molecular 22 mechanisms than only one agent at high dose with side-effect (Ohigashi and Murakami, 2004). 23 Accordingly, same authors previously showed synergistic effects of epigallocatechin gallate (0.04 $24 \mu \mathrm{M})$ and genistein $(2 \mu \mathrm{M})$ at low doses towards suppression of NO generation while both 25 compounds were antagonistic at high doses $(50 \mu \mathrm{M})$ and had no effect when tested alone 
1 (Murakami et al., 2003). In addition, choice of compounds with different mechanisms of action

2 should be "a prerequisite" to test synergicity (Ohigashi and Murakami, 2004).

3 Finally, besides the 4 main lipotropes that are choline, betaine, myo-inositol and metionine,

4 magnesium and B vitamins, we have showed that at least 14 other phytochemicals or groups of

5 phytochemicals may be considered as having a direct lipotropic effect (i.e. decreased hepatic TG

6 and/or lipid/fat contents) and/or as indirectly contributing to the overall lipotrope effect (e.g.

7 decreased lipogenic enzyme activities) (See Supplemental Tables 1-4). It seems, therefore, that as

8 for antioxidant phytomicronutrients, it would also exist within PBF a whole food package of

9 lipotropic phytomicronutrients for which the synergic action would be better than the action of

10 only one or two compounds; and for which physiological modes of action appear very diversified

11 such as the down- or up-regulation of gene expression, the inhibition of lipogenic enzymes and the

12 stimulation of FA oxidation enzymes, methyl donation for the synthesis of PL involved in

$13 \mathrm{VLDL} / \mathrm{LDL}$ exportation from liver, and/or action of enzyme co-factors.

\section{Several phytochemical properties to improve fatty liver}

18 In addition, since increased oxidative stress is also generally associated with fatty liver, both 19 lipotropes and antioxidants may synergistically contribute to alleviate hepatic steatosis. This is well 20 illustrated by the unrefined/virgin olive oil phytochemical package that is composed of several 21 compounds with complementary properties that all may contribute to protect from impaired 22 physiological functions associated with fatty liver: thus, Assy et al. proposed that the potential 23 proptective role of olive oil towards NAFLD may be attributed to the combined actions of phenolic 24 compounds (hydroxytyrosol, oleuropein, caffeic acid, o-coumaric acid, vanillic acid and 3,425 dihydroxyphenylethanol), oleic acid and squalene that exert anti-inflammatory, antioxidant and 26 immunomodulatory actions, that modulate transduction pathways, that regulate gene expression in 
1 liver regeneration, that inhibit HMG-CoA reductase and lipooxigenase, that change membrane

2 fluidity and/or that decrease RAS (belongs to GTPases, involved in receptor-mediated signal 3 transduction pathways) activation, all of them being involved in fatty liver development (Assy et 4 al., 2009).

5

6

\section{CONCLUSIONS AND PERSPECTIVES}

8

$9 \quad$ What compound should be considered as lipotropes?

11 If the lipotropic effect of some phytonutrients has been well studied in rats, paradoxically no 12 studies have defined the lipotrope content and lipotrope density of PBF, raw or processed. It is true 13 that the interest in betaine, choline and myo-inositol contents of PBF seems rather recent and 14 databases remain insufficient, especially for free myo-inositol.

Defining the lipotropic capacity of PBF involves defining what compounds should be considered as a lipotrope. Sensu stricto, it is a compound that decreased hepatic fat content, mainly TG content since TG are main constituent of excess fat deposits in steatosis (Adams et al., 2005; Araya et al., 2004). On such a basis, most of compounds cited in Supplemental Tables 1-4 are potential lipotropes for human nutrition, some being ubiquitous in PBF like betaine, choline, myoinositol, magnesium, B vitamins and polyphenols while other being specific of plant species like cysteine-containing compounds. Studies in rats have clearly demonstrated that betaine, choline, myo-inositol, methionine and carnitine have lipotropic effects and that physiological mechanisms of action differ from one compound to another (Figure 1 A-D). Then, results of Supplemental Tables clearly showed that niacin, pantothenic acid, folates may be considered as significantly contributing to the overall lipotropic effect. All these compounds have been cited as lipotrope in literature. Despite the absence of study, magnesium can be reasonably also considered as having a 
1 lipotropic action since indispensable as CoA cofactor allowing transformation of FA into acyl2 CoA. Otherwise, cobalamine (vitamin B12), cited as lipotrope in some studies, is the only 3 compound to be found exclusively in animal-based foods.

4 Concerning the other phytochemicals, to our knowledge, they have never been cited as 5 lipotropes in literature. From studies reported in Supplemental Tables and Tables 2 and 3 and 6 based on significant hepatic TG content reduction, one has considered that organosulfur 7 compounds, unsaturated FA (probably mainly n-3 PUFA such as $\alpha$-linolenic and/or n-9 MUFA like 8 oleic acid), acetic acid, melatonin, deoxynojirimycin, phytic acid, fiber, oligofructose, flavonoids, 9 lignans, stilbenes, curcumin and saponins may be considered as having a lipotropic effect. 10 However, except for phytic acid and lignans, further studies are undoubtedly necessary to confim 11 these first results, first in animal models, then in humans. For the remaining phytochemicals that 12 are phenolic acids, roryzanol, propionic acid, phytosterols, alkylresorcinol, policosanol and 13 tocotrienols, their ability to significantly reduce steatosis, hepatic TG and/or total lipid contents 14 remains to be demonstrated in both rats and humans. Their effect on hepatic cholesterol 15 metabolism and their ability to reduce its hepatic synthesis are more relevant than with TG.

16 While the antioxidant and hypolipidemic capacities of PBF have been extensively 17 investigated, the lipotropic capacity of PBF would therefore deserve more attention. Indeed, 18 similarly to increased oxidative stress and/or hyperlipidemia that have been shown to be involved 19 in the development of numerous metabolic and/or chronic diseases, fatty liver is also a common 20 symptom to several chronic diseases, especially in the first stage of pathology development.

23 The lack of human studies

25 Although numerous studies - mainly interventional - have underlined the ability of PBF to 26 positively affect some metabolic biomarkers, there is undoubtedly a lack of studies in humans that 
1 have investigated the lipotropic effect of complex PBF or of their phytonutrients as free 2 compounds. Thus, apart the few medical/clinical reports published in 1954 and 1964 concerning 3 patients that were administered lingtropic formula or tablets (Colson and Gallay, 1964; Nadeau et al., 1954; Navarranne et al. 1964; Warembourg and Bertrand, 1964) and the few reported studies

5 in choline-deficient subjects (Fischer et al., 2007,Zeisel et al., 1991) - notably as a result of total

6 parenteral nutrition (Buchman et al., 2001), in NAFLD patients administered either betaine 7 (Abdelmalek et al., 2001) or PUFA (Capanni et al., 2006; Spadaro et al., 2008), to our knowledge, 8 there is no intervention studies directly investigating the effect of complex PBF consumption on 9 the prevention of fatty liver development in humans. The first step might be to lead observational

10 studies and to search for associations between consumption of some foods, phytochemicals and/or 11 class of phytochemicals with NAFLD risk or prevalence. and/or legumes

The reasons for the rarity of human studies are unclear. One explanation may be linked to 13 the nature of technics that has to be used to diagnose hepatic stetaosis. Generally, the biomarker used in routine for evaluating liver injury in humans is the serum level of ALT. This level is then compared to those of alkaline phosphatase (ALP) and aspartate aminotransferase (AST) to help determine which form of liver disease is present, notably for hepatitis. But this test is not sufficiently specific to diagnose fatty liver. The most reliable test is biopsy, considered as the gold 18 standard to best characterizing steatosis, but it is invasive. It is therefore generally performed only 19 when more serious liver diseases are diagnosed. Alternatively, non-invasive technics like magnetic Spadaro et al., 2008) that allows estimating hepatic fat storage.

Other explanations for the lack of human studies may be based on the costliness of 24 intervention studies, or simply on the fact that the lipotropic property of phytochemicals has been 25 neglected or under-estimated to the benefit of their antioxidant and/or anticarcinogenic properties. 
Yet, the lipotrope supplements or complexes apparently constitute a large and lucrative

2 market targeted for people aiming at loosing weight via "fat burning" as indicated by

3 manufacturers. One may therefore reasonably suppose that it is very likely that intervention studies

4 have been performed in humans but that their results have not been published, since being perhaps

The contribution of metabolomics

\{Manna, 2010 \#20033\}: "Identification of Noninvasive Biomarkers for

essentially lead by private industry. Alcohol-Induced Liver Disease Using Urinary Metabolomics and the Ppara-null Mouse"

\{Cheng, 2010 \#22506\}: "Metabolomic study of the LDL receptor null mouse fed a high-fat diet reveals profound perturbations in choline metabolism that are shared with ApoE null mice" \{Barr, 2010 \#22914\} \{Griffin, 2006 \#10348\}: metabonomics for studying steatosis of liver \{Griffin, 2004 \#25160\}: metabonomics and fatty liver metabolism

Metabolomics is a quite recent set o \{Lazo, 2010 \#22481\}: Reduced steatosis through better lifestyle (moderate caloric restriction + exercise) is also possible : another biological fluids like urine, plasma alternative to lipotropes or a combination of both. \{Loftus, 2010 \#25931\}: liver and metabonomics

12 soluble, like from liver homogena 
1 glucose level and that serum lactate level tended to be lower at the limit of significance

2 (Subramanian et al., 2008). Based on these two markers, they have accurately classified 118/120

3 patients as control or NAFLD subjects (Subramanian et al., 2008). One may understand that by

4 unravelling new biomarkers in serum or urine through metabolomics, it will become quite

5 effective, easy and rapid to diagnose hepatic steatosis with a 100\%-reliability.

Otherwise, the few studies lead in animal models have allowed better understanding how

7 hepatic lipid metabolic pathways are involved in steatosis, which one are activated or depressed

8 and how lipidome or lipid profiles are modified compared to controls (Ginneken et al., 2007; $\overline{\overline{\bar{V}}}$

9 Griffin et al., 2007; Pilvi et al., 2008; Zivkovic et al., 2009). In these four studies, steatosis has and also how liver metabolite profiling changes upon high-cholesterol diet from simple steatosisi to steatohepatitis (Vinaixa et al (2010) (1) been provocked by starvation, high-fat diet, 1\% orotic acid supplementation and alcohol excess in 11 respectively mice (Ginneken et al., 2007; Pilvi et al., 2008), rats (Griffin et al., 2007) and minipigs 12 (Zivkovic et al., 2009). For example, in mice, while hepatic phosphatidylcholine content was 13 importantly reduced after 24 hours starvation, the appearance of a new putative biomarker of 14 steatosis was also observed; and it was identified as a 49:4-TG with an odd number of C atoms, 15 such odd TG being rare compounds (Ginneken et al., 2007). In the study with minipigs, Zivkovic 16 et al. showed that alcoholic steatosis is likely to notably result from alcohol suppressive efefct on 17 the phosphatidylethanolamine- $N$-methyltransferase pathway (Figure 2A) (Zivkovic et al., 2009).

18 Metabolomics appears therefore as a suitable complementary technic for studying effect of 19 phytochemicals on hepatic steatosis development or finding associations between levels of 20 phytochemical consumption and risk/prevalence or degree of NAFLD. That should allow leading 21 more human studies based on the simple measurement of new serum and/or urinary NAFLD 22 biomarkers. 
1 Last but not least issue is the absence of official database available for some of the lipotropic

2 compounds found PBF, notably free myo-inositol, carnitine, melatonin, organosulfur compounds, 3 acetic acid, oligofructose, curcumin and saponins. Data has to be found article by article - when

4 they exist! Concerning myo-inositol, the sole database is that of Clements and Darnell for total 5 myo-inositol (Clements and Darnell, 1980); however, it includes myo-inositol moieties from all 6 myo-inositol-derived compounds, notably phytic acid (myo-inositol hexakisphosphates) for which 7 the lipotropic effect has not been demonstrated in humans.

Concerning choline and betaine contents of foods, databases have been released only 9 recently between 2002 and 2008 (De Zwart et al., 2003; Sakamoto et al., 2002; Slow et al., 2005; 10 Zeisel et al., 2003), the most exhaustive and involving foods of different countries being that of 11 USDA released in 2008 (USDA, 2008).

Data for the methionine, magnesium, and B vitamin contents of PBF are obviously easily 13 available via notably the Souci et al. (Souci et al., 2008) and USDA (USDA, 2005a) databases by 14 food group.

Concerning polyphenols, databases and literature data become more and more numerous and accessible (Neveu et al., 2010; USDA, 2004, 2007, 2008; Wu et al., 2004b). The problem for polyphenols is that all polyphenols are probably not lipotropic: for example, as can be seen from

18 Supplemental Table 4, most striking effects have been obtained with catechins (a flavonoid) and 19 sesamin (a lignan) while no significant lipotropic effect has been reported for ferulic acid (phenolic 20 acid). This means that, ideally, one should determine the content in specific polyphenol food by 21 food. However, now, the recent Phenol-Explorer (Neveu et al. 2010) and USDA databases for the 22 flavonoid (USDA, 2007), proanthocyanidin (USDA, 2004) and isoflavone (USDA, 2008) contents 23 give such information for numerous PBF. In the end, as we discussed previously, one may also 24 make the approximation that the TPC content of PBF - that is generally measured in literature corresponds to one compound with a potential lipotropic effect. 


\section{ACKNOWLEDGMENTS}

2 Jean-François Martin (INRA, UMR 1019 Nutrition Humaine, F-63122 Saint Genès Champanelle,

3 France ; Clermont Université, UFR Médecine, UMR 1019 Nutrition Humaine, F-63000, Clermont-

4 Ferrand, France ; CRNH Auvergne, F-63000 Clermont-Ferrand, France) is gratefully 5 acknowledged for its helpful assistance in the elaboration of scattergrams. 


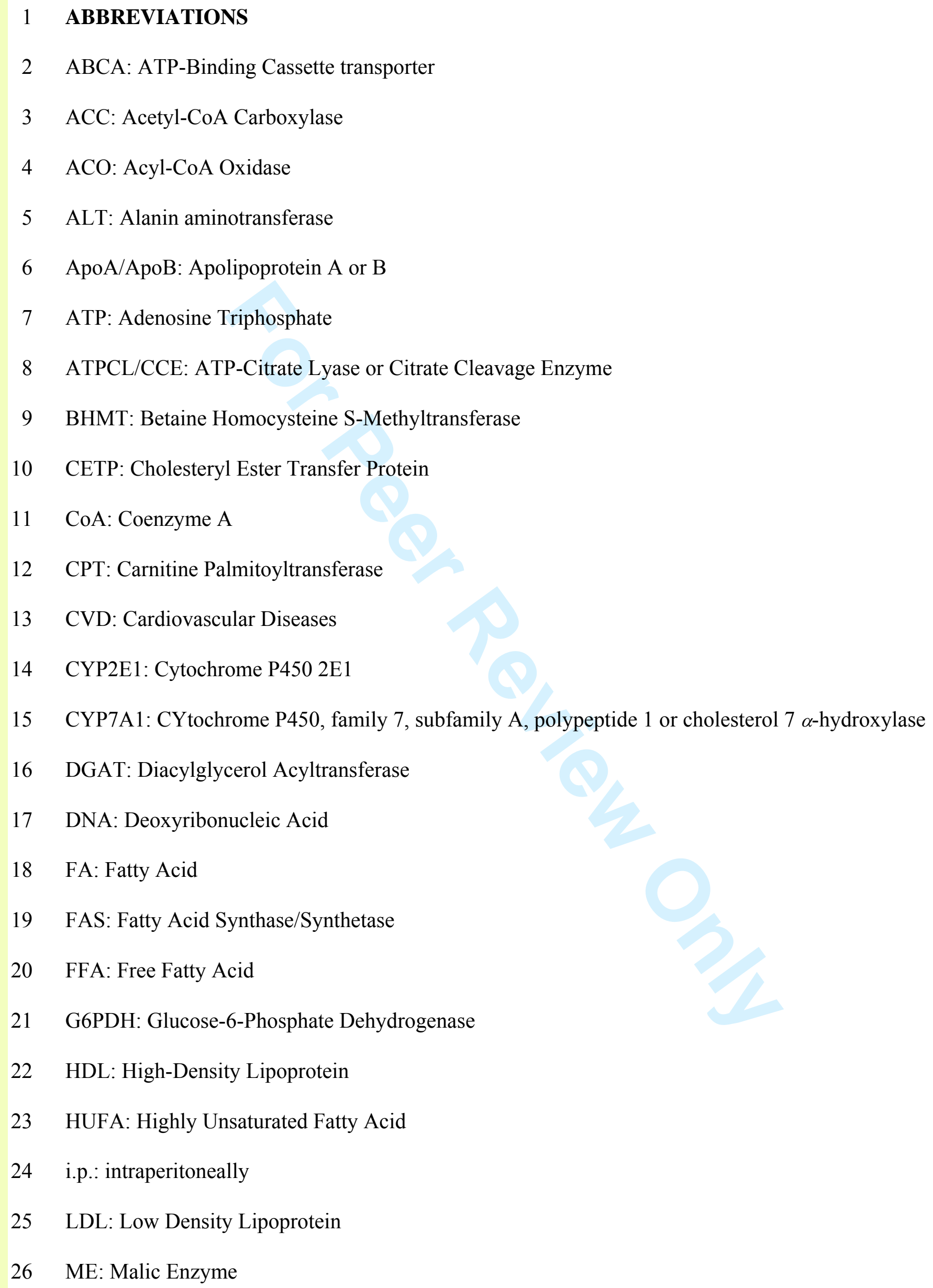


1 mRNA: Messenger Ribonucleic Acid

2 mtGPAT: mitochondrial Glycerol-3-Phosphate Acyltransferase

3 MTP: Microsomal triglyceride Transfert Protein

4 NAFL: Non-Alcoholic Fatty Liver

5 NAFLD: Non-Alcoholic Fatty Liver Disease

6 PABA: Para-Aminobenzoic Acid

7 PBF: Plant Based Foods

8 PL: Phospholipid

9 PPAR: Peroxisome Proliferator Activated Receptor

10 PUFA: Poly-Unsaturated Fatty Acid RS: Resistant Starch

11 SREBP: Sterol Regulatory Element Binding Protein

12 TG: Triglyceride

13 USDA: United States Department of Agriculture

14 VLDL: Very Low Density Lipoprotein 


\section{REFERENCES}

2 Abdelmalek, M. F., Angulo, P., Jorgensen, R. A., Sylvestre, P. B. and Lindor, K. D. (2001).

3 Betaine, a promising new agent for patients with nonalcoholic steatohepatitis: Results of a pilot 4 study. American Journal of Gastroenterology 96: 2711-2717.

Abe, M. and Kishino, Y. (1982). Pathogenesis of fatty liver in rats fed a high protein diet without 6 pyridoxine. Journal of Nutrition 112: 205-210.

7 Adams, L. A., Angulo, P. and Lindor, K. D. (2005). Nonalcoholic fatty liver disease. Canadian Medical Association Journal 172: 899-905.

9 Aghelli, N., Kabir, M., Berni-Canani, S., Petitjean, E., Boussairi, A., Luo, J., Bornet, F., Slama, G. 10 and Rizkalla, S. W. (1998). Plasma lipids and fatty acid synthase activity are regulated by short11 chain fructo-oligosaccharides in sucrose-fed insulin-resistant rats. Journal of Nutrition 128: 1283121288.

13 Alshatwi, A. A., Al Obaaid, M. A., Al Sedairy, S. A., Al-Assaf, A. H., Zhang, J. J. and Lei, K. Y. 14 (2010). Tomato powder is more protective than lycopene supplement against lipid peroxidation in 15 rats. Nutrition Research 30: 66-73.

16 Altschul, R., Hoffer, A. and Stephen, J. D. (1955). Influence of nicotinic acid on serum cholesterol 17 in man. Archives of Biochemistry and Biophysics 54: 558-559.

18 Alwayn, I. P. J., Gura, K., Nosé, V., Zaosche, B., Javid, P., Garza, J., Verbesey, J., Voss, S., Ollero, 19 M. andersson, C., Bistrian, B., Folkman, J. and Puder, M. (2005). Omega-3 fatty acid 20 supplementation prevents hepatic steatosis in a murine model of nonalcoholic fatty liver disease. 21 Pediatric Research 57: 445-452.

22 Andersen, D. B. and Holub, B. J. (1980). The relative response of hepatic lipids in the rat to graded 23 levels of dietary myo-inositol and other lipotropes. Journal of Nutrition 110: 496-504.

24 Andrieux-Domont, C. and Le van, H. (1970). Influence of magnesium on enzymatic synthesis of 25 coenzyme a. Comptes Rendus des Séances de la Société de Biologie et de ses Filiales 164: 29226296. 
1 Angulo, P. (2002). Nonalcoholic fatty liver disease. New England Journal of Medicine 346: 122121231.

3 Angulo, P. and Lindor, K. D. (2001). Treatment of nonalcoholic fatty liver: present and emerging $4 \quad$ therapies. Seminars in Liver Disease 21: 81-88.

Araya, J., Rodrigo, R., Videla, L. A., Thielemann, L., Orellana, M., Pettinelli, P. and Poniachik, J. 6 (2004). Increase in long-chain polyunsaturated fatty acid $n-6 / n-3$ ratio in relation to hepatic 7 steatosis in patients with non-alcoholic fatty liver disease. Clinical Science 106: 635-643.

Arner, P. (1999). Catecholamine-induced lipolysis in obesity. International Journal of Obesity 23: $9 \quad 10-13$.

Aronov, D. M., Keenan, J. M., Akhmedzhanov, N. M., Tikhomirova, E. A., Perova, N. V., Olferiev,

Arvidson, G. and Borgström, B. (1963). Studies in choline deficiency. Fate of injected 1- ${ }^{14}$ Cpalmitic acid and fatty acid spectra in fasting and refed rats. Proceedings of the Society for Experimental Biology and Medicine 112: 676-679.

Arvidson, G. A. E. and Asp, N.-G. (1982). Hepatic free choline and betaine and the utilization of dietary protein in the choline-deficient rat. Annals of Nutrition and Metabolism 26: 12-17.

Ashakumary, L., Rouyer, I., Takahashi, Y., Ide, T., Fukuda, N., Aoyama, T., Hashimoto, T., Mizugaki, M. and Sugano, M. (1999). Sesamin, a sesame lignan, is a potent inducer of hepatic fatty acid oxidation in the rat. Metabolism 48: 1303-1313.

Assy, N., Nassar, F., Nasser, G. and Grosovski, M. (2009). Olive oil consumption and non22 alcoholic fatty liver disease. World Journal of Gastroenterology 15: 1809-1815.

23 Audet, A. and Lupien, P. J. (1974). Triglyceride metabolism in pyridoxine-deficient rats. Journal of $24 \quad$ Nutrition 104: 91-100.

Azzi, A. and Stocker, A. (2000). Vitamin e: Non-antioxidant roles. Progress in Lipid Research 39: $231-255$ 
1 Badmaev, V., Majeed, M. and Conte, A. A. (2002). Open field, physician controlled clinical 2 evaluation of a botanical weight loss formula based on garcinia cambogia derived (-)hydroxycitric 3 acid. NutraCos 1: 10-14.

4 Baggenstoss, A., Christensen, N., Berge, K. G., Baldus, W. P., Spiekerman, R. and Ellefson, R. D. 5 (1967). Fine structural changes in liver in hypercholesteremic patients receiving long-term $6 \quad$ nicotinic acid therapy. Mayo Clinic Proceedings 42: 385-399.

7 Baker, H., Frank, O., Zetterman, R. and Hutner, S. H. (1977). Possible explanation of potentiation 8 of hepatic steatosis induced by high nicotinic-acid, choline and ethionine. Nutrition Reports 9 International 15: 607-618.

10 Baker, H., Luisada-Opper, A., Sorrell, M. F., Thomson, A. D. and Frank, O. (1973). Inhibition by 11 nicotinic acid of hepatic steatosis and alcohol dehydrogenase in ethanol-treated rats. Experimental 12 and Molecular Pathology 19: 106-112.

13 Balkan, J., Oztezcan, S., Kucuk, M., Cevikbas, U., Kocak-Toker, N. and Uysal, M. (2004). The 14 effect of betaine treatment on triglyceride levels and oxidative stress in the liver of ethanol-treated 15 guinea pigs. Experimental and Toxicologic Pathology 55: 505-509.

16 Ball, C. R. (1964). Actions of betaine, carnitine and choline on pattern of hepatic liposis in mice fed 17 high-fat low-protein diet. Anatomical Record 149: 677-689.

18 Baquet, A., Maisin, L. and Hue, L. (1991). Swelling of rat hepatocytes activates acetyl-CoA 19 carboxylase in parallel to glycogen synthase. Biochemical Journal 278: 887-890.

20 Barak, A. J., Beckenhauer, H. C., Badakhsh, S. and Tuma, D. J. (1997). The effect of betaine in 21 reversing alcoholic steatosis. Alcoholism: Clinical and Experimental Research 21: 1100-1102.

22 Barak, A. J., Beckenhauer, H. C. and Tuma, D. J. (1996a). Betaine effects on hepatic methionine 23 metabolism elicited by short-term ethanol feeding. Alcohol 13: 483-486.

24 Barak, A. J., Beckenhauer, H. C. and Tuma, D. J. (1996b). Betaine, ethanol and the liver: A review. 25 Alcohol 13: 395-398. 
1 Barak, A. J., Beckenhauer, H. C., Tuma, D. J. and Badakhsh, S. (1987). Effects of prolonged 2 ethanol feeding on methionine metabolism in rat-liver. Biochemistry and Cell Biology-Biochimie 3 Et Biologie Cellulaire 65: 230-233.

4 Barbieri, B., Papadogiannakis, N., Eneroth, P. and Olding, L. B. (1995). Arachidonic-acid is a 5 preferred acetyl donor among fatty-acids in the acetylation of p-aminobenzoic acid by human 6 lymphoid-cells. Biochimica Et Biophysica Acta-Lipids and Lipid Metabolism 1257: 157-166.

7 Barclay, J. A. and Cooke, W. T. (1945). Hepatorenal syndrome treated with choline chloride. The 8 Lancet 246: 458-460.

9 Barr, I. G., Sjölander, A. and Cox, J. C. (1998). Iscoms and other saponin based adjuvants. 10 Advanced Drug Delivery Reviews 32: 247-271.

11 Bartsch, H. and Nair, J. (2006). Chronic inflammation and oxidative stress in the genesis and 12 perpetuation of cancer: Role of lipid peroxidation, DNA damage and repair. Langenbeck's 13 Archives of Surgery 391: 499-510.

14 Bazzano, L. A., Li, T. Y., Joshipura, K. J. and Hu, F. B. (2008). Intake of fruit, vegetables and fruit 15 juices and risk of diabetes in women. Diabetes Care 31: 1311-1317.

16 Beach, D. C. and Flick, P. K. (1982). Early effect of myoinositol deficiency on fatty-acid synthetic 17 enzymes of rat-liver. Biochimica Et Biophysica Acta 711: 452-459.

18 Beams, A. J. (1946). The treatment of cirrhosis of the liver with choline and cystine. Journal of the 19 American Medical Association 130: 190-194.

20 Becker, R., Wheeler, E. L., Lorenz, K., Stafford, A. E., Grosjean, O. K., Betschart, A. A. and 21 Saunders, R. M. (1981). A compositional study of amaranth grain. Journal of Food Science 46: $22 \quad 1175-1180$.

23 Beeston, A. W. and Channon, H. J. (1936). Cystine and the dietary production of fatty livers. 24 Biochemical Journal 30: 280-284. 
1 Beher, W. T. and Anthony, W. L. (1955). Effects of beta-sitosterol and ferric chloride on 2 accumulation of cholesterol in mouse liver. Proceedings of the Society for Experimental Biology 3 and Medicine 90: 223-225.

4 Berg, P. (1959). Role of magnesium in acetyl coenzyme a formation by acetothiokinase. Science 5 129: $895-896$.

6 Berlow, S., Bachman, P. and Berry, G. (1989). Betaine therapy in homocystinemia. Brain 7 Dysfunction 2: 10-24.

8 Best, C. H. (1935). The lipotropic effect of protein. Nature 135: 821-822.

9 Best, C. H. (1934). The role of the liver in the metabolism of carbohydrate and fat. III - the 10 deposition of liver fat. The Lancet 223: 1274-1277.

11 Best, C. H., Hartroft, W. S., Lucas, C. C. and Ridout, J. H. (1955). Effects of dietary protein, 12 lipotropic factors and re-alimentation on total hepatic lipids and their distribution. British Medical 13 Journal 1: 1439-1444.

14 Best, C. H. and Huntsman, M. E. (1932). The effects of the components of lecithine upon 15 deposition of fat in the liver. Journal of Physiology 75: 405-412.

16 Best, C. H. and Huntsman, M. E. (1935). The effect of choline on the liver fat of rats in various 17 states of nutrition. Journal of Physiology 83: 255-274.

18 Best, C. H., Lucas, C. C., Ridout, J. H. and Patterson, J. M. (1950). Dose-response curves in the 19 estimation of potency of lipotropic agents. Journal of Biological Chemistry 186: 317-329.

20 Best, C. H. and Ridout, J. H. (1940). The lipotropic action of methionine. Journal of Physiology 97 : $21 \quad 489-494$.

22 Best, M. M. and Duncan, C. H. (1956). Effects of sitosterol on the cholesterol concentration in 23 serum and liver in hypothyroidism. Circulation 14: 344-348.

24 Beveridge, J. M. R., Lucas, C. C. and O'Grady, M. K. (1945). The effect of dietary proteins and 25 amino acids on liver fat. Journal of Biological Chemistry 160: 505-518. 
1 Beylot, M. (2005). Effects of inulin-type fructans on lipid metabolism in man and in animal models. British Journal of Nutrition 93: S163-S168.

3 Bitar, K. and Reinhold, J. G. (1972). Phytase and alkaline phosphatase activities in intestinal 4 mucosae of rat, chicken, calf and man. Biochimica et Biophysica Acta 268: 442-452.

Blanchard, G., Paragon, B. M., Milliat, F. and Lutton, C. (2002). Dietary 1-carnitine supplementation in obese cats alters carnitine metabolism and decreases ketosis during fasting and induced hepatic lipidosis. Journal of Nutrition 132: 204-210.

Bloch, K. and Rittenberg, D. (1942). On the utilization of acetic acid for cholesterol formation. Journal of Biological Chemistry 145: 625-636.

10 Borgschulte, G., Kathirvel, E., Herrera, M., French, S. W., Morgan, T. R., Morgan, K. and 11 Bottiglieri, T. (2008). Betaine treatment reverses insulin resistance and fatty liver disease without 12 reducing oxidative stress or endoplasmic reticulum stress in an animal model of nafld. 13 Gastroenterology 134: A414-A415.

14 Bortolotti, M., Kreis, R., Debard, C., Cariou, B., Faeh, D., Chetiveaux, M., Ith, M., Vermathen, P., 15 Stefanoni, N., Le, K.-A., Schneiter, P., Krempf, M., Vidal, H., Boesch, C. and Tappy, L. (2009). 16 High protein intake reduces intrahepatocellular lipid deposition in humans. American Journal of 17 Clinical Nutrition 90: 1002-1010.

18 Bose, M., Lambert, J. D., Ju, J., Reuhl, K. R., Shapses, S. A. and Yang, C. S. (2008). The major 19 green tea polyphenol, (-)-epigallocatechin-3-gallate, inhibits obesity, metabolic syndrome and 20 fatty liver disease in high-fat-fed mice. Journal of Nutrition 138: 1677-1683.

21 Bourdin, B., Adenier, H. and Perrin, Y. (2007). Camitine is associated with fatty acid metabolism in 22 plants. Plant Physiology and Biochemistry 45: 926-931.

23 Boyd, J. N., Sherman, W. K., Graham, E. S., Graham, T. C. and Tennant, B. C. (1986). A 24 comparison of the response of woodchucks and rats to variations in dietary lipotrope and protein 25 content. Journal of Nutrition 116: 2044-2054. 
1 Brandsch, C., Shukla, A., Hirche, F., Stangl, G. I. and Eder, K. (2006). Effect of proteins from beef, 2 pork and turkey meat on plasma and liver lipids of rats compared with casein and soy protein. $3 \quad$ Nutrition 22: 1162-1170.

4 Brandt, K., Langhans, W., Geary, N. and Leonhardt, M. (2006). Beneficial and deleterious effects 5 of hydroxycitrate in rats fed a high-fructose diet. Nutrition 22: 905-912.

6 Briggs, G. M. and Daft, F. S. (1955). Water-soluble vitamins. I. Vitamin B12, folic acid, choline $7 \quad$ and para-aminobenzoic acid. Annual Review of Biochemistry 24: 339-392.

8 Brindle, J. T., Antti, H., Holmes, E., Tranter, G., Nicholson, J. K., Bethell, H. W. L., Clarke, S., 9 Schofield, P. M., McKilligin, E., Mosedale, D. E. and Grainger, D. J. (2002). Rapid and 10 noninvasive diagnosis of the presence and severity of coronary heart disease using H-1-NMR11 based metabonomics. Nature Medicine 8: 1439-1444.

12 Broun, G. O. (1948). Treatment of hepatic cirrhosis. Postgraduate Medicine 4: 203.

13 Broun, G. O. and Meuther, R. O. (1942). The treatment of hepatic cirrhosis with choline chloride 14 and a diet low in fat and cholesterol. Journal of the American Medical Association 118: 1403.

15 Brouwer, I. A., van Dusseldorp, M., West, C. E., Meyboom, S., Thomas, C. M. G., Duran, M., Hof, 16 K. H. V., Eskes, T., Hautvast, J. and Steegers-Theunissen, R. P. M. (1999). Dietary folate from 17 vegetables and citrus fruit decreases plasma homocysteine concentrations in humans in a dietary 18 controlled trial. Journal of Nutrition 129: 1135-1139.

19 Brouwers, M., Bilderbeek-Beckers, M. A. L., Georgieva, A. M., van der Kallen, C. J. H., van 20 Greevenbroek, M. M. J. and de Bruin, T. W. A. (2005). Fatty liver in hypertriglyceridemic 21 subjects with familial combined hyperlipidemia: Importance of visceral fat. Atherosclerosis 22 Supplements 6: 30-30.

23 Brufau, G., Canela, M. A. and Rafecas, M. (2008). Phytosterols: Physiologic and metabolic aspects 24 related to cholesterol-lowering properties. Nutrition Research 28: 217-225. 
1 Bruno, R. S., Dugan, C. E., Smyth, J. A., DiNatale, D. A. and Koo, S. I. (2008). Green tea extract

2 protects leptin-deficient, spontaneously obese mice from hepatic steatosis and injury. Journal of $3 \quad$ Nutrition 138: 323-331.

4 Brunt, E. M., Janney, C. G., Di Bisceglie, A. M., Neuschwander-Tetri, B. A. and Bacon, B. R. (1999). Nonalcoholic steatohepatitis: A proposal for grading and staging the histological lesions. American College of Gastroenterology 94: 2467-2474.

7 Buchman, A. L., Ament, M. E., Sohel, M., Dubin, M., Jenden, D. J., Roch, M., Pownall, H., Farley, 8 W., Awal, M. and Ahn, C. (2001). Choline deficiency causes reversible hepatic abnormalities in 9 patients receiving parenteral nutrition: Proof of a human choline requirement: A placebo10 controlled trial. Journal of Parenteral and Enteral Nutrition 25: 260-268.

11 Buchman, A. L., Dubin, M. D., Moukarzel, A. A., Jenden, D. J., Roch, M., Rice, K. M., Gornbein, 12 J. and Ament, M. E. (1995). Choline deficiency: A cause of hepatic steatosis during parenteral 13 nutrition that can be reversed with intravenous choline supplementation. Hepatology 22: 1399141403.

15 Buijsse, B., Feskens, E. J. M., Schulze, M. B., Forouhi, N. G., Wareham, N. J., Sharp, S., Palli, D., 16 Tognon, G., Halkjaer, J., Tjonneland, A., Jakobsen, M. U., Overvad, K., van der A, D. L., Du, H. 17 D., Sorensen, T. I. A. and Boeing, H. (2009). Fruit and vegetable intakes and subsequent changes 18 in body weight in european populations: Results from the project on diet, obesity and genes 19 (diogenes). American Journal of Clinical Nutrition 90: 202-209.

20 Burton, L. E. and Wells, W. W. (1977). Characterization of the lactation-dependent fatty liver in 21 myo-inositol deficient rats. Journal of Nutrition 107: 1871-1883.

22 Busserolles, J., Gueux, E., Rock, E., Demigne, C., Mazur, A. and Rayssiguier, Y. (2003). 23 Oligofructose protects against the hypertriglyceridemic and pro-oxidative effects of a high 24 fructose diet in rats. Journal of Nutrition 133: 1903-1908. 
1 Caballero, F., Fernandez, A., Fernandez-Checa, J. C. and Garcia-Ruiz, C. (2008). Methionine 2 deficiency accounts for the liver damage observed in a nutritional model of nonalcoholic 3 steatohepatitis. Journal of Hepatology 48: 917.

4 Caldas, T., Demont-Caulet, N., Ghazi, A. and Richarme, G. (1999). Thermoprotection by glycine 5 betaine and choline. Microbiology 145: 2543-2548.

6 Calhoun, W. K., Bechtel, W. G. and Bradley, W. B. (1958). The vitamin content of wheat, flour and 7 bread. Cereal Chemistry 35: 350-359.

8 Calhoun, W. K., Hepburn, F. N. and Bradley, W. B. (1960). The distribution of the vitamins of $9 \quad$ wheat in commercial mill products. Cereal Chemistry 37: 755 - 761.

10 Cantoni, G. L. (1951). Methylation of nicotinamide with a soluble enzyme system from rat liver. 11 Journal of Biological Chemistry 189: 203-216.

12 Capanni, M., Calella, F., Biagini, M. R., Genise, S., Raimondi, L., Bedogni, G., Svegliati-Baroni, 13 G., Sofi, F., Milani, S., Abbate, R., Surrenti, C. and Casini, A. (2006). Prolonged n-3 14 polyunsaturated fatty acid supplementation ameliorates hepatic steatosis in patients with non15 alcoholic fatty liver disease: A pilot study. Alimentary Pharmacology and Therapeutics 23: 1143161151.

17 Carlson, L. A. (1963). Studies on effect of nicotinic acid on catecholamine stimulated lipolysis in 18 adipose tissue in vitro. Acta Medica Scandinavica 173: 719-722.

19 Carroll, C. and Williams, L. (1982). Choline deficiency in rats as influenced by dietary energy20 sources. Nutrition Reports International 25: 773-782.

21 Carter, C. W. and Hockaday, T. D. (1962). Liver lipids and ketone-body formation in rats deficient 22 in pantothenate. Biochemical Journal 84: 275-280.

23 Carter, C. W. and Phizackerley, P. J. R. (1951). The influence of pyridoxine on fat metabolism in 24 the rat. Biochemical Journal 49: 227-232.

25 Castelao, J. E. and Gago-Dominguez, M. (2008). Risk factors for cardiovascular disease in women: 26 Relationship to lipid peroxidation and oxidative stress. Medical Hypotheses 71: 39-44. 
1 Castelein, H., Gulick, T., Declercq, P. E., Mannaerts, G. P., Moore, D. D. and Baes, M. I. (1994).

2 The peroxisome proliferator activated receptor regulates malic enzyme gene expression. Journal 3 of Biological Chemistry 269: 26754-26758.

4 Catala, A., Zvara, A., Puskas, L. G. and Kitajka, K. (2007). Melatonin-induced gene expression 5 changes and its preventive effects on adriamycin-induced lipid peroxidation in rat liver. Journal 6 of Pineal Research 42: 43-49.

7 Catolla Cavalcanti, A. and Levis, F. (1950). Steatosis of the liver due to phosphorus. I. Lipotropic action of pantothenic acid and of its combination with meso-inositol. Archivio per le Scienze Mediche (Torino) 90: 529-541.

Causi, N., Romano, A. and Galfano, G. (1958). Coenzyme a in rat liver during administration of pantothenic acid and nicotinamide. Bollettino-Societa Italiana Biologia Sperimentale (Napoli) 34: $12 \quad 163-164$.

13 Chahl, J. S. and Kratzing, C. C. (1966a). Environmental temperature and choline requirement in rats 14 .I. Choline deficiency in rats at various temperatures. Journal of Lipid Research 7: 17-21.

15 Chahl, J. S. and Kratzing, C. C. (1966b). Environmental temperature and choline requirements in 16 rats .2. Choline and methionine requirements for lipotropic activity. Journal of Lipid Research 7: $17 \quad 22-26$.

18 Chan, J. M., Wang, F. and Holly, E. A. (2007). Whole grains and risk of pancreatic cancer in a large 19 population-based case-control study in the san francisco bay area, california. American Journal of 
1 Channon, H. J. and Wilkinson, H. (1935). Protein and the dietary production of fatty livers. 2 Biochemical Journal 29: 350-356.

3 Chapman, M. J., Le Goff, W., Guerin, M. and Kontush, A. (2010). Cholesteryl ester transfer 4 protein: At the heart of the action of lipid-modulating therapy with statins, fibrates, niacin and 5 cholesteryl ester transfer protein inhibitors. European Heart Journal 31: 149-164.

6 Chatenoud, L., Tavani, A., La Vecchia, C., Jacobs, D. R., Jr., Negri, E., Levi, F. and Franceschi, S. 7 (1998). Whole grain food intake and cancer risk. International Journal of Cancer 77: 24-28.

Chen, Q. (2004). Determination of phytic acid and inositol pentakisphosphates in foods by highperformance ion chromatography. Journal of Agricultural and Food Chemistry 52: 4604-4613.

Cho, Y. Y., Kwon, E. Y., Kim, H. J., Park, Y. B., Lee, K. T., Park, T. and Choi, M. S. (2009). Low trans structured fat from flaxseed oil improves plasma and hepatic lipid metabolism in apo e-/mice. Food and Chemical Toxicology 47: 1550-1555.

Choi, J. S., Koh, I. U., Jung, M. H. and Song, J. (2007). Effects of three different conjugated linoleic acid preparations on insulin signalling, fat oxidation and mitochondrial function in rats fed a high-fat diet. British Journal of Nutrition 98: 264-275.

Christman, J. K., Chen, M.-L., Sheikhnejad, G., Dizik, M., Abileah, S. and Wainfan, E. (1993). Methyl deficiency, DNA methylation and cancer: Studies on the reversibility of the effects of a lipotrope-deficient diet. Journal of Nutritional Biochemistry 4: 672-680.

Cicero, A. F. G. and Gaddi, A. (2001). Rice bran oil and gamma-oryzanol in the treatment of hyperlipoproteinaemias and other conditions. Phytotherapy Research 15: 277-289.

Clarke, S. D. (2001). Nonalcoholic steatosis and steatohepatitis.: I. Molecular mechanism for polyunsaturated fatty acid regulation of gene transcription. American Journal of PhysiologyGastrointestinal and Liver Physiology 281: G865-869.

Clarke, S. D., Romsos, D. R. and Leveille, G. A. (1977). Differential effects of dietary methyl esters of long-chain saturated and polyunsaturated fatty acids on rat liver and adipose tissue lipogenesis. Journal of Nutrition 107: 1170-1181. 
1 Clements, R., Jr and Darnell, B. (1980). Myo-inositol content of common foods: Development of a 2 high-myo- inositol diet. American Journal of Clinical Nutrition 33: 1954-1967.

3 Clements, R. and Reynertson, R. (1977). Myoinositol metabolism in diabetes mellitus. Effect of $4 \quad$ insulin treatment. Diabetes 26: 215-221.

5 Colson, J. A. and Gallay, C. (1964). Trial treatment of hepatic metabolic disorders by an original 6 formula containing mainly ornithine combined with various classical lipotropic substances.

$7 \quad$ Toulouse Med 65: 207-229.

8 Cortez-Pinto, H., Camilo, M. E., Baptista, A., De Oliveira, A. G. and De Moura, M. C. (1999).

9 Non-alcoholic fatty liver: Another feature of the metabolicsyndrome? Clinical Nutrition 18: $353-$ 10358

11 Cortez-Pinto, H., Jesus, L., Barros, H., Lopes, C., Moura, M. C. and Camilo, M. E. (2006). How 12 different is the dietary pattern in non-alcoholic steatohepatitis patients? Clinical Nutrition 25: $13 \quad 816-823$.

14 Craig, S. A. S. (2004). Betaine in human nutrition. American Journal of Clinical Nutrition 80: 53915549.

16 da Costa, K.-A., Garner, S. C., Chang, J. and Zeisel, S. H. (1995). Effects of prolonged (1 year) 17 choline deficiency and subsequent re-feeding of choline on 1,2-sn-diradylglycerol, fatty acids and 18 protein kinase c in rat liver. Carcinogenesis 16: 327-334.

19 Dahiru, D. and Obidoa, O. (2009). Effect of aqueous extract of ziziphus mauritiana leaf on 20 cholesterol and triglyceride levels in serum and liver of rats administered alcohol. Pakistan $21 \quad$ Journal of Nutrition 8: 1884-1888.

22 Dalton, T. A. and Berry, R. S. (1992). Hepatotoxicity associated with sustained-release niacin. 23 American Journal of Medicine 93: 102-104.

24 Daubioul, C., Rousseau, N., Demeure, R., Gallez, B., Taper, H., Declerck, B. and Delzenne, N. 25 (2002). Dietary fructans, but not cellulose, decrease triglyceride accumulation in the liver of obese 26 zucker fa/fa rats. Journal of Nutrition 132: 967-973. 
1 Daubioul, C. A., Taper, H. S., De Wispelaere, L. D. and Delzenne, N. M. (2000). Dietary 2 oligofructose lessens hepatic steatosis, but does not prevent hypertriglyceridemia in obese zucker 3 rats. Journal of Nutrition 130: 1314-1319.

4 Day, C. P. and James, O. F. W. (1998a). Hepatic steatosis: Innocent bystander or guilty party? 5 Hepatology 27: 1463-1466.

6 Day, C. P. and James, O. F. W. (1998b). Steatohepatitis: A tale of two "hits"? Gastroenterology 7 114: $842-845$.

8 de Melo, D. S., Correa, A. D., Marcos, F. C. A., de Sousa, R. V., de Abreu, C. M. P. and dos 9 Santos, C. D. (2008). Effects of cassava leaves flour on the ast, alt, alp enzymes activity and 10 hepatic lipids of wistar rats. Ciencia e Tecnologia de Alimentos 28: 32-37.

11 de Munter, J. S., Hu, F. B., Spiegelman, D., Franz, M. and van Dam, R. M. (2007). Whole grain, 12 bran and germ intake and risk of type 2 diabetes: A prospective cohort study and systematic 13 review. PLoS Medicine 4: e261.

14 de Zwart, F. J., Slow, S., Payne, R. J., Lever, M., George, P. M., Gerrard, J. A. and Chambers, S. T. 15 (2003). Glycine betaine and glycine betaine analogues in common foods. Food Chemistry 83 : $16 \quad 197-204$.

17 Decombaz, J., Gmuender, B., Sierro, G. and Cerretelli, P. (1992). Muscle carnitine after strenuous 18 endurance exercise. Journal of Applied Physiology 72: 423-427.

19 Degrace, P., Demizieux, L., Du, Z. Y., Gresti, J., Caverot, L., Djaouti, L., Jourdan, T., Moindrot, B., 20 Guilland, J. C., Hocquette, J. F. and Clouet, P. (2007). Regulation of lipid flux between liver and 21 adipose tissue during transient hepatic steatosis in carnitine-depleted rats. Journal of Biological 22 Chemistry 282: 20816-20826.

23 Delzenne, N. M. and Daubioul, C. (2000). Dietary fructans and lipid metabolism: Building a bridge 24 from the colon to the liver. Recent Research Developments in Nutrition 3: 227-238.

25 Delzenne, N. M. and Kok, N. N. (1999). Biochemical basis of oligofructose-induced hypolipidemia 26 in animal models. Journal of Nutrition 129: 1467-. 
1 Demigné, C., Morand, C., Levrat, M.-A., Besson, C., Moundras, C. and Rémésy, C. (1995). Effect 2 of propionate on fatty acid and cholesterol synthesis and on acetate metabolism in isolated rat 3 hepatocytes. British Journal of Nutrition 74: 209-219.

4 Di Nunzio, M., van Deursen, D., Verhoeven, A. J. M. and Bordoni, A. (2010). N-3 and n-6 5 polyunsaturated fatty acids suppress sterol regulatory element binding protein activity and 6 increase flow of non-esterified cholesterol in HepG2 cells. British Journal of Nutrition 103: 161$7 \quad 167$.

8 Doherty, J. F., Adam, E. J., Griffin, G. E. and Golden, M. H. (1992). Ultrasonographic assessment 9 of the extent of hepatic steatosis in severe malnutrition. Archives of Disease in Childhood 67: $10 \quad 1348-1352$.

11 Drill, V. A. (1954). Lipotropic effects of vitamin-b12 and other factors. Annals of the New York 12 Academy of Sciences 57: 654-663.

13 du Vigneaud, V., Chandler, J. P., Cohn, M. and Brown, G. B. (1940). The transfer of the methyl 14 group from methionine to choline and creatine. Journal of Biological Chemistry 134: 787-788.

15 du Vigneaud, V., Cohn, M., Chandler, J. P., Schenck, J. R. and Simmonds, S. (1941). The 16 utilization of the methyl group of methionine in the biological synthesis of choline and creatine. 17 Journal of Biological Chemistry 140: 625-641.

18 Dulloo, A. G., Duret, C., Rohrer, D., Girardier, L., Mensi, N., Fathi, M., Chantre, P. and 19 Vandermander, J. (1999). Efficacy of a green tea extract rich in catechin polyphenols and caffeine 20 in increasing 24-h energy expenditure and fat oxidation in humans. American journal of Clinical $21 \quad$ Nutrition 70: 1040-1045.

22 Dumas, M. E., Barton, R. H., Toye, A., Cloarec, O., Blancher, C., Rothwell, A., Fearnside, J., 23 Tatoud, R., Blanc, V., Lindon, J. C., Mitchell, S. C., Holmes, E., McCarthy, M. I., Scott, J., 24 Gauguier, D. and Nicholson, J. K. (2006). Metabolic profiling reveals a contribution of gut 25 microbiota to fatty liver phenotype in insulin-resistant mice. Proceedings of the National 26 Academy of Sciences of the United States of America 103: 12511-12516. 
1 Eastwood, M. and Mowbray, L. (1976). The binding of the components of mixed micelle to dietary 2 fiber. American journal of Clinical Nutrition 29: 1461-1467.

3 Eastwood, M. A. (1975). Vegetable dietary fiber - potent pith. Royal Society of Health Journal 95: $4 \quad 188-190$.

5 Eastwood, M. A. (1999). Interaction of dietary antioxidants in vivo: How fruit and vegetables 6 prevent disease? Qjm-an International Journal of Medicine 92: 527-530.

7 Eastwood, M. A. and Girdwood, R. H. (1968). Lignin - a bile-salt sequestrating agent. Lancet 2: $8 \quad 1170-1172$.

9 Eastwood, M. A. and Hamilton, D. (1968). Studies on adsorption of bile salts to non-absorbed 10 components of diet. Biochimica et Biophysica Acta 152: 165-173.

11 Eckstein, H. C. (1952). Dietary essential amino acids and the liver lipide content of young white 12 rats. Journal of Biological Chemistry 195: 167-174.

13 Eikelboom, J. W., Lonn, E., Genest, J., Hankey, G. and Yusuf, S. (1999). Homocyst(e)ine and 14 cardiovascular disease: A critical review of the epidemiologic evidence. Annals of Internal $15 \quad$ Medicine 131: 363-375.

16 El-Enein, A. M. A., Hafez, Y. S., Salem, H. and Abdel, M. (1983). Role of nicotinic-acid and 17 inositol hexa nicotinate as anti cholesterolemic and anti lipemic agents. Nutrition Reports 18 International 28: 899-912.

19 Engel, R. W. (1942). The relation of b-vitamins and dietary fat to the lipotropic action of choline. $20 \quad$ Journal of Nutrition 24: 175-185.

21 Esfandiari, F., Villanueva, J. A., Wong, D. H., French, S. W. and Halsted, C. H. (2005). Chronic 22 ethanol feeding and folate deficiency activate hepatic endoplasmic reticulum stress pathway in 23 micropigs. American Journal of Physiology-Gastrointestinal and Liver Physiology 289: G54-63.

24 Esfandiari, F., You, M., Villanueva, J. A., Wong, D. H., French, S. W. and Halsted, C. H. (2007). S25 adenosylmethionine attenuates hepatic lipid synthesis in micropigs fed ethanol with a folate26 deficient diet. Alcoholism: Clinical and Experimental Research 31: 1231-1239. 
1 Etchason, J. A., Miller, T. D., Squires, R. W., Allison, T. G., Gau, G. T., Marttila, J. K. and Kottke, 2 B. A. (1991). Niacin-induced hepatitis - a potential side-effect with low-dose time-release niacin. $3 \quad$ Mayo Clinic Proceedings 66: 23-28.

4 Failey, R. B. and Childress, R. H. (1962). The effect of para-aminobenzoic acid on the serum $5 \quad$ cholesterol level in man. American Journal of Clinical Nutrition 10: 158-162.

6 Fang, Y. Z., Yang, S. and Wu, G. Y. (2002). Free radicals, antioxidants and nutrition. Nutrition 18: $7 \quad 872-879$.

8 Fardet, A. (2009). New hypotheses for the health protective mechanisms of whole-grain cereals:

$9 \quad$ What is beyond fibre? Nutrition research Reviews In press.

10 Fardet, A., Canlet, C., Gottardi, G., Lyan, B., Remesy, C., Mazur, A., Paris, A. and A., S. (2007). 11 Whole grain and refined wheat flours show distinct metabolic profiles in rats as assessed by a ${ }^{1} \mathrm{H}-$ 12 NMR-based metabonomic approach. Journal of Nutrition 4: 923-929.

13 Fardet, A., Rock, E. and Rémésy, C. (2008). Is the in vitro antioxidant potential of whole-grain 14 cereals and cereal products well reflected in vivo? Journal of Cereal Science 48: 258-276.

15 Felmlee, M. A., Woo, G., Simko, E., Krol, E. S., Muir, A. D. and Alcorn, J. (2009). Effects of the 16 flaxseed lignans secoisolariciresinol diglucoside and its aglycone on serum and hepatic lipids in 17 hyperlipidaemic rats. British Journal of Nutrition 102: 361-369.

18 Fidanza, A., Decicco, A., Fiorilli, G. and Bruno, C. (1970). Liver lipids of pantothenic acid and 19 polynsaturated fatty acids deficient rats. Bollettino Della Societa Italiana Di Biologia $20 \quad$ Sperimentale 46: 684-686.

21 Figge, H. L., Figge, J., Souney, P. F., Mutnick, A. H. and Sacks, F. (1988). Nicotinic-acid - a 22 review of its clinical use in the treatment of lipid disorders. Pharmacotherapy 8: 287-294.

23 Fischer, L. M., daCosta, K. A., Kwock, L., Stewart, P. W., Lu, T.-S., Stabler, S. P., Allen, R. H. and 24 Zeisel, S. H. (2007). Sex and menopausal status influence human dietary requirements for the 25 nutrient choline. American journal of Clinical Nutrition 85: 1275-1285. 
1 Flight, I. and Clifton, P. (2006). Cereal grains and legumes in the prevention of coronary heart 2 disease and stroke: A review of the literature. European Journal of Clinical Nutrition 60: 114531159.

4 Fomenko, A. I., Shushevich, S. I. and Khalmuradov, A. G. (1979). Inhibition of activity of acetyl 5 coenzyme a carboxylase from chicken liver by nicotinic-acid and its derivatives. Biokhimiya 44: $6 \quad 1005-1009$.

7 Freedman, A. M., Mak, I. T., Stafford, R. E., Dickens, B. F., Cassidy, M. M., Muesing, R. A. and 8 Weglicki, W. B. (1992). Erythrocytes from magnesium-deficient hamsters display an enhanced 9 susceptibility to oxidative stress. American Journal of Physiology 262: C1371-C1375.

10 Freeland, K. R., Wilson, C. and Wolever, T. M. S. (2010). Adaptation of colonic fermentation and 11 glucagon-like peptide-1 secretion with increased wheat fibre intake for 1 year in 12 hyperinsulinaemic human subjects. British Journal of Nutrition 103: 82-90.

13 Fritz, I. B. (1959). Action of carnitine on long chain fatty acid oxidation by liver. American Journal 14 of Physiology- 197: 297-304.

15 Fritz, I. B. and Dupont, P. (1957). Ineffectiveness of carnitine as a choline substitute in the 16 prevention of fatty livers of rats maintained on a choline-deficient diet. American Journal of 17 Physiology-Gastrointestinal and Liver Physiology 190: 453-454.

18 Fromenty, B., Grimbert, S., Mansouri, A., Beaugrand, M., Erlinger, S., Rötig, A. and Pessayre, D. 19 (1995). Hepatic mitochondrial DNA deletion in alcoholics: Association with microvesicular 20 steatosis. Gastroenterology 108: 193-200.

21 Fromenty, B. and Pessayre, D. (1995). Inhibition of mitochondrial beta-oxidation as a mechanism 22 of hepatotoxicity. Pharmacology and Therapeutics 67: 101-154.

23 Fukuwatari, T., Morikawa, Y., Sugimoto, E. and Shibata, K. (2002). Effects of fatty liver induced 24 by niacin-free diet with orotic acid on the metabolism of tryptophan to niacin in rats. Bioscience, 25 Biotechnology, and Biochemistry 66: 1196-1204. 
1 Fux, M., Levine, J., Aviv, A. and Belmaker, R. (1996). Inositol treatment of obsessive-compulsive 2 disorder. American Journal of Psychiatry 153: 1219-1221.

3 Galli, A., Pinaire, J., Fischer, M., Dorris, R. and Crabb, D. W. (2001). The transcriptional and DNA 4 binding activity of peroxisome proliferator-activated receptor $\hat{\mathrm{i}} \pm$ is inhibited by ethanol 5 metabolism. Journal of Biological Chemistry 276: 68-75.

6 Ganji, S. H., Tavintharan, S., Zhu, D. M., Kamanna, V. S. and Kashyap, M. L. (2002). Niacin non7 competitively inhibits hepatocyte diacylglycerol acyltransferase, a key enzyme for triglyceride synthesis. Arteriosclerosis Thrombosis and Vascular Biology 22: P238.

9 Garfinkel, L. and Garfinkel, D. (1985). Magnesium regulation of the glycolytic pathway and the 10 enzymes involved. Magnesium 4: 60-72.

11 Gastaldelli, A., Kozakova, M., Hojlund, K., Flyvbjerg, A., Favuzzi, A., Mitrakou, A. and Balkau, B. 12 (2009). Fatty liver is associated with insulin resistance, risk of coronary heart disease and early 13 atherosclerosis in a large european population. Hepatology 49: 1537-1544.

14 Gavin, G. and McHenry, E. W. (1940). The b vitamins and fat metabolism. Iii. The effects of 15 vitamin B6 upon liver and body fat. Journal of Biological Chemistry 132: 41-46.

16 Gavin, G. and McHenry, E. W. (1941a). Inositol: A lipotropic factor. Journal of Biological 17 Chemistry 139: 485.

18 Gavin, G. and McHenry, E. W. (1941b). The effect of biotin upon fat synthesis and metabolism. 19 Journal of Biological Chemistry 141: 619-625.

20 Gaylor, J. L., Hardy, R. W. F. and Baumann, C. A. (1960). Effects of nicotinic acid and related 21 compounds on sterol metabolism in the chick and rat. Journal of Nutrition 70: 293-301.

22 Gebhardt, R. and Beck, H. (1996). Differential inhibitory effects of garlic-derived organosulfur 23 compounds on cholesterol biosynthesis in primary rat hepatocyte cultures. Lipids 31: 1269-1276.

24 Gershoff, S. N. and Gottlieb, L. S. (1964). Pantothenic acid deficiency in cats. Journal of Nutrition 25 82: $135-138$. 
1 Gey, K. F. (1998). Vitamins e plus c and interacting conutrients required for optimal health: A 2 critical and constructive review of epidemiology and supplementation data regarding 3 cardiovascular disease and cancer. Biofactors 7: 113-174.

4 Ghoshal, A. K. and Farber, E. (1984). The induction of liver cancer by dietary deficiency of choline 5 and methionine without added carcinogens. Carcinogenesis 5: 1367-1370.

6 Gillis, M. B. and Norris, L. C. (1951). The effect of vitamin B12 on the response of chicks to 7 betaine and choline. Journal of Nutrition 43: 295-302.

8 Ginneken, V. v., Verhey, E., Poelmann, R., Ramakers, R., Dijk, K. W. v., Ham, L., Voshol, P., 9 Havekes, L., Eck, M. v. and Greef, J. v. d. (2007). Metabolomics (liver and blood profiling) in a 10 mouse model in response to fasting: A study of hepatic steatosis. Biochimica et Biophysica Acta, $11 \quad$ Molecular and Cell Biology of Lipids 1771: 1263-1270.

12 Goelz, S., Vogelstein, B., Hamilton, SR and Feinberg, A. (1985). Hypomethylation of DNA from 13 benign and malignant human colon neoplasms. Science 228: 187-190.

14 Goheen, S., Larkin, E. and Rao, G. (1983). Severe fatty liver in rats fed a fat-free ethanol diet and 15 its prevention by small amounts of dietary arachidonate. Lipids 18: 285-290.

16 Gotoh, N., Nagao, K., Onoda, S., Shirouchi, B., Furuya, K., Nagai, T., Mizobe, H., Ichioka, K., 17 Watanabe, H., Yanagita, T. and Wada, S. (2009). Effects of three different highly purified n-3 18 series highly unsaturated fatty acids on lipid metabolism in c57bl/ksj-db/db mice. Journal of 19 Agricultural and Food Chemistry 57: 11047-11054.

20 Gouni-Berthold, I. and Berthold, H. K. (2002). Policosanol: Clinical pharmacology and therapeutic 21 significance of a new lipid-lowering agent. American Heart Journal 143: 356-365.

22 Graham, I. M., Daly, L. E., Refsum, H. M., Robinson, K., Brattstrom, L. E., Ueland, P. M., PalmaReis, R. J., Boers, G. H., Sheahan, R. G., Israelsson, B., Uiterwaal, C. S., Meleady, R., McMaster, D., Verhoef, P., Witteman, J., Rubba, P., Bellet, H., Wautrecht, J. C., de Valk, H. W., Sales Luis, A. C., Parrot-Rouland, F. M., Tan, K. S., Higgins, I., Garcon, D., Medrano, M. J., Candito, M., Evans, A. E. and Andria, G. (1997). Plasma homocysteine as a risk factor for vascular disease. 
1 The european concerted action project. Journal Of the American Medical Association 277: 177521781.

3 Greenwood, M. R. C., Cleary, M. P., Gruen, R., Blase, D., Stern, J. S., Triscari, J. and Sullivan, A. 4 C. (1981). Effect of (-)-hydroxycitrate on development of obesity in the zucker obese rat. $5 \quad$ American Journal of Physiology 240: E72-E78.

6 Griffin, J. L., Scott, J. and Nicholson, J. K. (2007). The influence of pharmacogenetics on fatty liver 7 disease in the wistar and kyoto rats: A combined transcriptomic and metabonomic study. Journal 8 of Proteome Research 6: 54-61.

9 Griffin, J. L. and Vidal-Puig, A. (2008). Current challenges in metabolomics for diabetes research: 10 A vital functional genomic tool or just a ploy for gaining funding? Physiological Genomics 34: 1115.

12 Griffith, W. H. and Mulford, D. J. (1941a). Choline metabolism. Vi. Hemorrhagic degeneration and 13 the labile methyl supply. Journal of the American Chemical Society 63: 929-932.

14 Griffith, W. H. and Mulford, D. J. (1941b). Choline metabolism: Vii. Some dietary factors affecting 15 the incidence and severity of hemorrhagic degeneration in young rats. Journal of Nutrition 21: $16 \quad 633-646$.

17 Grundy, S., Mok, H., Zech, L. and Berman, M. (1981). Influence of nicotinic acid on metabolism of 18 cholesterol and triglycerides in man. Journal of Lipid Research 22: 24-36.

19 Guehring, R. R., Hurley, L. S. and Morgan, A. F. (1952). Cholesterol metabolism in pantothenic 20 acid deficiency. Journal of Biological Chemistry 197: 485-493.

21 Guggenheim, K. and Olson, R. E. (1952). Studies of lipogenesis in certain b-vitamin deficiencies. 22 Journal of Nutrition 48: 345-358.

23 Guzmán, M., Velasco, G., Castro, J. and Zammit, V. A. (1994). Inhibition of carnitine 24 palmitoyltransferase i by hepatocyte swelling. FEBS Letters 344: 239-241.

25 Haines, D. S. M. and Mookerjea, S. (1965). Impairment of triglyceride transport from liver in 26 choline deficiency. Canadian Journal of Biochemistry 43: 507-520. 
1 Halliday, N. (1938). Fatty livers in vitamin B6 deficient rats. Journal of Nutrition 16: 285-290.

2 Halsted, C. H., Villanueva, J. A., Devlin, A. M., Niemelä, O., Parkkila, S., Garrow, T. A., Wallock, 3 L. M., Shigenaga, M. K., Melnyk, S. and James, S. J. (2002). Folate deficiency disturbs hepatic 4 methionine metabolism and promotes liver injury in the ethanol-fed micropig. Proceedings of the $5 \quad$ National Academy of Sciences of the United States of America 99: 10072-10077.

6 Hammond, L. E., Lewin, T. M., Schwerbrock, N. M. J., Maeda, N. and Coleman, R. A. (2003). 7 Reduced liver and heart triacylglycerol content in mitochondrial glycerol-3-phosphate acyltransferase -/- mice following high-sucrose feeding. FASEB Journal 17: A1315-A1315.

9 Handler, P. (1944). The effect of excessive nicotinamide feeding on rabbits and guinea pigs. 10 Journal of Biological Chemistry 154: 203-206.

11 Handler, P. and Dann, W. J. (1942). The inhibition of rat growth by nicotinamide. Journal of 12 Biological Chemistry 146: 357-368.

13 Hanje, A. J., Fortune, B., Song, M., Hill, D. and McClain, C. (2006). The use of selected nutrition 14 supplements and complementary and alternative medicine in liver disease. Nutrition in Clinical 15 Practice 21: 255-272.

16 Hanson, A. D. and Hitz, W. D. (1982). Metabolic responses of mesophytes to plant water deficits. 17 Annual Review of Plant Physiology 33: 163-203.

18 Hanson, A. D., May, A. M., Grumet, R., Bode, J., Jamieson, G. C. and Rhodes, D. (1985). Betaine 19 synthesis in chenopods - localization in chloroplasts. Proceedings of the National Academy of 20 Sciences of the United States of America 82: 3678-3682.

21 Hanson, A. D. and Wyse, R. (1982). Biosynthesis, translocation and accumulation of betaine in 22 sugar beet and its progenitors in relation to salinity. Plant Physiology 70: 1191-1198.

23 Hara, H., Haga, S., Aoyama, Y. and Kiriyama, S. (1999). Short-chain fatty acids suppress 24 cholesterol synthesis in rat liver and intestine. Journal of Nutrition 129: 942-948.

25 Harland, B. F. and Oberleas, D. (1987). Phytate in foods. World Review of Nutrition and Dietetics $26 \quad 52: 235-259$. 
1 Harper, A. E., Benton, D. A., Winje, M. E. and Elvehjem, C. A. (1954a). On the lipotropic action of 2 protein. Journal of Biological Chemistry 209: 171-177.

3 Harper, A. E., Monson, W. J., Benton, D. A., Winje, M. E. and Elvehjem, C. A. (1954b). Factors 4 other than choline which affect the deposition of liver fat. Journal of Biological Chemistry 206: $5 \quad 151-158$

6 Harper, A. E., Monson, W. J., Benton, D. A. and Elvehjem, C. A. (1953). The influence of protein 7 and certain amino acids, particularly threonine, on the deposition of fat in the liver of the rat. .

Hartfiel, W. and Kirchner, I. (1973). The importance of nicotinic acid and its effect on the liver fat content of laying hens. Archiv fur Geflugelkunde 37: 114-117.

Häussinger, D. (1996). The role of cellular hydration in the regulation of cell function. Biochemical Journal 313: 697-710.

Hayashi, E., Maeda, T. and Tomita, T. (1974a). Effect of myoinositol deficiency on lipidmetabolism in rats .1. Alteration of lipid-metabolism in myoinositol deficient rats. Biochimica et Biophysica Acta 360: 134-145.

Hayashi, E., Maeda, T. and Tomita, T. (1974b). Effect of myoinositol deficiency on lipidmetabolism in rats .2. Mechanism of triacylglycerol accumulation in liver of myoinositol-deficient rats. Biochimica Et Biophysica Acta 360: 146-155.

He, K., Hu, F. B., Colditz, G. A., Manson, J. E., Willett, W. C. and Liu, S. (2004). Changes in intake of fruits and vegetables in relation to risk of obesity and weight gain among middle-aged women. International Journal of Obesity 28: 1569-1574.

Helfrich, A. and Bettmer, J. (2004). Determination of phytic acid and its degradation products by ion-pair chromatography (IPC) coupled to inductively coupled plasma-sector field-mass spectrometry (ICP-SF-MS). Journal of Analytical Atomic Spectrometry 19: 1330-1334.

Henning, S. M. and Swendseid, M. E. (1996). The role of folate, choline and methionine in carcinogenesis induced by methyl-deficient diets. In: Dietary fats, lipids, hormones and 
1 tumorigenesis: new horizons in basic research. pp. 143-155. Heber, D. and Kritchevsky, D., Eds., 2 Plenum Press, New York.

3 Heymsfield, S. B., Allison, D. B., Vasselli, J. R., Pietrobelli, A., Greenfield, D. and Nunez, C. 4 (1998). Garcinia cambogia (hydroxycitric acid) as a potential antiobesity agent: A randomized 5 controlled trial. Journal of the American Medical Association 280: 1596-1600.

6 Hitz, W. D., Ladyman, J. A. R. and Hanson, A. D. (1982). Betaine synthesis and accumulation in 7 barley during field water-stress. Crop Science 22: 47-54.

8 Horbowicz, M., Brenac, P. and Obendorf, R. L. (1998). Fagopyritol B1, O- $\alpha$-D-galactopyranosyl-(1 9 ->-2)-D-chiro-inositol, a galactosyl cyclitol in maturing buckwheat seeds associated with 10 desiccation tolerance. Planta 205: 1-11.

11 Horbowicz, M. and Obendorf, R. L. (1994). Seed desiccation tolerance and storability: Dependence 12 on flatulence-producing oligosaccharides and cyclitols? - review and survey. Seed Science 13 Research 4: 385-405.

14 Horev-Azaria, L., Eliav, S., Izigov, N., Pri-Chen, S., Mirelman, D., Miron, T., Rabinkov, A., 15 Wilchek, M., Jacob-Hirsch, J., Amariglio, N. and Savion, N. (2009). Allicin up-regulates cellular 16 glutathione level in vascular endothelial cells. European Journal of Nutrition 48: 67-74.

17 Hosomi, R., Fukunaga, K., Arai, H., Nishiyama, T. and Yoshida, M. (2009). Effects of dietary fish 18 protein on serum and liver lipid concentrations in rats and the expression of hepatic genes 19 involved in lipid metabolism. Journal of Agricultural and Food Chemistry 57: 9256-9262.

20 Hough, V. H., Monahan, E. P., Li, T. W. and Freeman, J. (1943). The effect of choline and cystine 21 on the serum phosphatase and hepatic dye clearance of dogs maintained on deficient diets. 22 American Journal of Physiology 642: 642-651.

23 Hozawa, A., Kuriyama, S., Nakaya, N., Ohmori-Matsuda, K., Kakizaki, M., Sone, T., Nagai, M., 24 Sugawara, Y., Nitta, A., Tomata, Y., Niu, K. and Tsuji, I. (2009). Green tea consumption is 25 associated with lower psychological distress in a general population: The Ohsaki Cohort 2006 26 Study. American Journal of Clinical Nutrition 90: 1390-1396. 
1 Hsu, C.-C., Yen, H.-f., Yin, M.-C., Tsai, C.-M. and Hsieh, C.-H. (2004). Five cysteine-containing 2 compounds delay diabetic deterioration in balb/ca mice. Journal of Nutrition 134: 3245-3249.

3 Hsu, C.-L. and Yen, G.-C. (2008). Phenolic compounds: Evidence for inhibitory effects against 4 obesity and their underlying molecular signaling mechanisms. Molecular Nutrition and Food $5 \quad$ Research 52: 53-61.

$6 \mathrm{Hu}, \mathrm{M}$. C. C. (1975). Lipotropic action of carnitine in a low protein diet from plant sources. $7 \quad$ Dissertation Abstracts International, B 36: 655.

8 Hue, L. (1994). Control of liver carbohydrate and fatty-acid metabolism by cell-volume. 9 Biochemical Society Transactions 22: 505-508.

10 Hung, H. C., Joshipura, K. J., Jiang, R., Hu, F. B., Hunter, D., Smith-Warner, S. A., Colditz, G. A., 11 Rosner, B., Spiegelman, D. and Willett, W. C. (2004). Fruit and vegetable intake and risk of 12 major chronic disease. Journal of the National Cancer Institute 96: 1577-1584.

13 Hussein, O., Grosovski, M., Lasri, E., Svalb, S., Ravid, U. and Assy, N. (2007). Monounsaturated 14 fat decreases hepatic lipid content in non-alcoholic fatty liver disease in rats. World Journal of 15 Gastroenterology 13: 361-368.

16 Ide, T., Ashakumary, L., Takahashi, Y., Kushiro, M., Fukuda, N. and Sugano, M. (2001). Sesamin, 17 a sesame lignan, decreases fatty acid synthesis in rat liver accompanying the down-regulation of 18 sterol regulatory element binding protein-1. Biochimica et Biophysica Acta-Molecular and Cell 19 Biology of Lipids 1534: 1-13.

20 Ide, T., Hong, D. D., Ranasinghe, P., Takahashi, Y., Kushiro, M. and Sugano, M. (2004). 21 Interaction of dietary fat types and sesamin on hepatic fatty acid oxidation in rats. Biochimica et 22 Biophysica Acta-Molecular and Cell Biology of Lipids 1682: 80-91.

23 Ikeda, M., Uno, Y., Iwai, M., Sato, H., Kawabe, H. and Sakakibara, B. (1992). Effects of the 24 inositol-deficient diet on the development of fatty liver lipogenic enzyme activities and plasma 25 lipid levels in germ-free and conventional mice. Vitamins (Kyoto) 66: 43-49. 
1 Ingraham, L. L. and Green, D. E. (1958). Role of magnesium in enzyme-catalyzed syntheses 2 involving adenosine triphosphate. Science 128: 310-312.

3 Institute of Medicine and Food and Nutrition Board (1998). Niacin. In: Dietary reference intakes 4 for thiamin, riboflavin, niacin, vitamin B6, folate, vitamin B12, pantothenic acid, biotin and 5 choline, pp. 123-149. National Academy Press, Washington DC.

6 Iqbal, T. H., Lewis, K. O. and Cooper, B. T. (1994). Phytase activity in the human and rat small$7 \quad$ intestine. Gut 35: 1233-1236.

8 Ishihara, K., Oyaizu, S., Onuki, K., Lim, K. and Fushiki, T. (2000). Chronic (-)-hydroxycitrate 9 administration spares carbohydrate utilization and promotes lipid oxidation during exercise in 10 mice. Journal of Nutrition 130: 2990-2995.

11 Iwami, K., Sakakibara, K. and Ibuki, F. (1986). Involvement of post-digestion hydrophobic 12 peptides in plasma cholesterol-lowering effect of dietary plant-proteins. Agricultural and 13 Biological Chemistry 50: 1217-1222.

14 Jacobs, D. R., Jr andersen, L. F. and Blomhoff, R. (2007). Whole-grain consumption is associated 15 with a reduced risk of noncardiovascular, noncancer death attributed to inflammatory diseases in 16 the iowa women's health study. American Journal of Clinical Nutrition 85: 1606-1614.

17 Jacobs, D. R., Jr., Marquart, L., Slavin, J. and Kushi, L. H. (1998). Whole-grain intake and cancer: 18 An expanded review and meta-analysis. Nutrition and Cancer 30: 85-96.

19 Jaenicke, L. and Rudiger, H. (1971). Formation of methionine methyl groups. Federation 20 Proceedings 30: 160-166.

21 James, O. F. W. and Day, C. P. (1998). Non-alcoholic steatohepatitis (nash): A disease of emerging 22 identity and importance. Journal of Hepatology 29: 495-501.

23 Jamil, H., Chu, C.-H., K . Dickson, J., , Jr., Chen, Y., Yan, M., Biller, S. A., Gregg, R. E., Wetterau, 24 J. R. and Gordon, D. A. (1998). Evidence that microsomal triglyceride transfer protein is limiting 25 in the production of apolipoprotein b-containing lipoproteins in hepatic cells. Journal of Lipid $26 \quad$ Research 39: 1448-1454. 
1 Jeong, Y., Choi, Y., Kim, D., Park, S., Yoon, J., Kwon, S., Park, E. and Park, K. (2005).

2 Cytoprotective effect of green tea extract and quercetin against hydrogen peroxide-induced 3 oxidative stress. Archives of Pharmacal Research 28: 1251-1256.

4 Jin, F. Y., Kamanna, V. S. and Kashyap, M. L. (1999). Niacin accelerates intracellular apob 5 degradation by inhibiting triacylglycerol synthesis in human hepatoblastoma (HepG2) cells. 6 Arteriosclerosis Thrombosis and Vascular Biology 19: 1051-1059.

7 Jin, F.-Y., Kamanna, V. S. and Kashyap, M. L. (1996). Niacin accelerates intracellular post8 translational apolipoprotein $\mathrm{b}$ translocation/degradation by inhibiting triacylglycerol synthesis in 9 human hepatoblastoma (hep g2) cells. Circulation 94: I149.

10 Jin, F.-Y., Kamanna, V. S. and Kashyap, M. L. (1997). Niacin decreases removal of high-density 11 lipoprotein apolipoprotein A-I but not cholesterol ester by Hep G2 cells : Implication for reverse 12 cholesterol transport. Arteriosclerosis, Thrombosis, and Vascular Biology 17: 2020-2028.

13 Johnston, P. V., Kopaczyk, K. C. and Kummerow, F. A. (1961). Effect of pyridoxine deficiency on 14 fatty acid composition of carcass and brain lipids in the rat. Journal of Nutrition 74: 96-102.

15 Kahlon, T. S., Saunders, R. M., Sayre, R. N., Chow, F. I., Chiu, M. M. and Betschart, A. A. (1992). 16 Cholesterol-lowering effects of rice bran and rice bran oil fractions in hypercholesterolemic 17 hamsters. Cereal Chemistry 69: 485-489.

18 Kang, D. H., Jung, E. Y., Chang, U. J., Bae, S. H. and Suh, H. J. (2007). Psyllium husk combined 19 with hydroxycitrate reduces body weight gain and body fat in diet-induced obese rats. Nutrition $20 \quad$ Research 27: 349-355.

21 Kaplan, N. O. and Lipmann, F. (1948). The assay and distribution of coenzyme a. Journal of 22 Biological Chemistry 174: 37-44.

23 Karanth, J. and Jeevaratnam, K. (2009). Effect of dietary lipid, carnitine and exercise on lipid 24 profile in rat blood, liver and muscle. Indian Journal of Experimental Biology 47: 748-753. 
1 Kashyap, M. L., Jin, F. Y. and Kamanna, V. S. (1997). Niacin, by inhibiting both fatty acid 2 synthesis and triacylglycerol formation, augments apo b degradation in Hep G2 cells. $3 \quad$ Atherosclerosis 134: 127-127.

4 Katayama, T. (1995). Effect of dietary-sodium phytate on the hepatic and serum levels of lipids and 5 on the hepatic activities of nadph-generating enzymes in rats fed on sucrose. Bioscience 6 Biotechnology and Biochemistry 59: 1159-1160.

7 Katayama, T. (1997a). Dietary phytic acid acts on hepatic lipid metabolism in a similar manner as 8 dietary myo-inositol: is phytic acid a vitamin-like substance? Recent Research Developments in Agricultural and Biological Chemistry 1: 321-330.

10 Katayama, T. (1997b). Effects of dietary myo-inositol or phytic acid on hepatic concentrations of 11 lipids and hepatic activities of lipogenic enzymes in rats fed on corn starch or sucrose. Nutrition $12 \quad$ Research 17: 721-728.

13 Keaney, J. F., Larson, M. G., Vasan, R. S., Wilson, P. W. F., Lipinska, I., Corey, D., Massaro, J. 14 M., Sutherland, P., Vita, J. A. and Benjamin, E. J. (2002). Obesity as a source of systemic 15 oxidative stress: Clinical correlates of oxidative stress in the framingham study. Circulation 106: $16 \quad 467-467$.

17 Keim, N. L. and Mares-Perlman, J. A. (1984). Development of hepatic steatosis and essential fatty 18 acid deficiency in rats with hypercaloric, fat-free parenteral nutrition. Journal of Nutrition 114: $19 \quad 1807-1815$

20 Kelley, B., Totter, J. R. and Day, P. L. (1950). The lipotropic effect of folic acid on rats receiving 21 various purified diets. Journal of Biological Chemistry 187: 529-535.

22 Kersten, S., Seydoux, J., Peters, J. M., Gonzalez, F. J., Desvergne, B. a. and Wahli, W. (1999). 23 Peroxisome proliferator-activated receptor $\alpha$ mediates the adaptive response to fasting. Journal of 24 Clinical Investigation 103: 1489-1498.

25 Khairallah, E. A. and Wolf, G. (1965). Growth-promoting and lipotropic effect of carnitine in rats 26 fed diets limited in protein and methionine. Journal of Nutrition 87: 469-476. 
1 Khanal, T., Choi, J. H., Hwang, Y. P., Chung, Y. C. and Jeong, H. G. (2009). Protective effects of via the activation of amp-dependent protein kinase. Food and Chemical Toxicology 47: 27492754.

Khor, H. T., Chieng, D. Y. and Ong, K. K. (1995). Tocotrienols inhibit liver HMG CoA reductase activity in the guinea pig. Nutrition Research 15: 537-544.

Kim, H. K., Jeong, T. S., Lee, M. K., Park, Y. B. and Choi, M. S. (2003). Lipid-lowering efficacy of hesperetin metabolites in high-cholesterol fed rats. Clinica Chimica Acta 327: 129-137.

Kim, J. I., Kim, J. C., Joo, H. J., Jung, S. H. and Kim, J. J. (2005). Determination of total chiroinositol content in selected natural materials and evaluation of the antihyperglycemic effect of pinitol isolated from soybean and carob. Food Science and Biotechnology 14: 441-445.

Kisters, K., Spieker, C., Tepel, M. and Zidek, W. (1993). New data about the effects of oral physiological magnesium supplementation on several cardiovascular risk factors (lipids and blood pressure). Magnesium Research 6: 355-360.

Klaus, S., Pultz, S., Thone-Reineke, C. and Wolfram, S. (2005). Epigallocatechin gallate attenuates diet-induced obesity in mice by decreasing energy absorption and increasing fat oxidation. International Journal of Obesity 29: 615-623.

Ko, W.-C., Shih, C.-M., Lai, Y.-H., Chen, J.-H. and Huang, H.-L. (2004). Inhibitory effects of flavonoids on phosphodiesterase isozymes from guinea pig and their structure-activity relationships. Biochemical Pharmacology 68: 2087-2094.

Koh-Banerjee, P., Franz, M., Sampson, L., Liu, S., Jacobs, D. R., Jr., Spiegelman, D., Willett, W. and Rimm, E. (2004). Changes in whole-grain, bran and cereal fiber consumption in relation to 8y weight gain among men. American Journal of Clinical Nutrition 80: 1237-1245.

Koh-Banerjee, P. and Rimm, E. B. (2003). Whole grain consumption and weight gain: a review of the epidemiological evidence, potential mechanisms and opportunities for future research. Proceedings of the Nutrition Society 62: 25-29. 
1 Koivisto, M., Valta, P., Höckerstedt, K. and Lindgren, L. (2002). Magnesium depletion in chronic 2 terminal liver cirrhosis. Clinical Transplantation 16: 325-328.

3 Kok, N., Roberfroid, M. and Delzenne, N. (1996a). Dietary oligofructose modifies the impact of 4 fructose on hepatic triacylglycerol metabolism. Metabolism 45: 1547-1550.

5 Kok, N., Roberfroid, M., Robert, A. and Delzenne, N. (1996b). Involvement of lipogenesis in the 6 lower VLDL secretion induced by oligofructose in rats. British Journal of Nutrition 76: 881-890.

7 Kok, N. N., Morgan, L. M., Williams, C. M., Roberfroid, M. B., Thissen, J.-P. and Delzenne, N. M. 8 (1998). Insulin, glucagon-like peptide 1, glucose-dependent insulinotropic polypeptide and 9 insulin-like growth factor $\mathrm{i}$ as putative mediators of the hypolipidemic effect of oligofructose in 10 rats. Journal of Nutrition 128: 1099-1103.

11 Kolonel, L. N., Hankin, J. H., Whittemore, A. S., Wu, A. H., Gallagher, R. P., Wilkens, L. R., John, 12 E. M., Howe, G. R., Dreon, D. M., West, D. W. and Paffenbarger, R. S., Jr. (2000). Vegetables, 13 fruits, legumes and prostate cancer: A multiethnic case-control study. Cancer Epidemiology, 14 Biomarkers and Prevention 9: 795-804.

15 Kondo, T., Kishi, M., Fushimi, T. and Kaga, T. (2009). Acetic acid upregulates the expression of 16 genes for fatty acid oxidation enzymes in liver to suppress body fat accumulation. Journal of 17 Agricultural and Food Chemistry 57: 5982-5986.

18 Koseki, M., Seki, H., Kazama, M., Kitabatake, N. and Tochikura, T. (1991). Effects of pectin and 19 lard on the production of short-chain fatty-acids in the cecum, on the growth of colonic bacteria 20 and on the liver cholesterol level in rats. Agricultural and Biological Chemistry 55: 1441-1448.

21 Kotaki, A., Sakurai, T., Kobayashi, M. and Yagi, K. (1968). Studies on myoinositol. IV. Effect of 22 myoinositol on the cholesterol metabolism of rats suffering from experimental fatty liver. Journal of Vitaminology (Kyoto) 14.

Koteish, A. and Diehl, A. M. (2001). Animal models of steatosis. Seminars in Liver Disease 21: 89104. 
1 Kovacs, E. M. R., Westerterp-Plantenga, M. S., de Vries, M., Brouns, F. and Saris, W. H. M. 2 (2001a). Effects of 2-week ingestion of (-)-hydroxycitrate and (-)-hydroxycitrate combined with 3 medium-chain triglycerides on satiety and food intake. Physiology and Behavior 74: 543-549.

4 Kovacs, E. M. R., Westerterp-Plantenga, M. S. and Saris, W. H. M. (2001b). The effects of 2-week 5 ingestion of (-)-hydroxycitrate and (-)-hydroxycitrate combined with medium-chain triglycerides 6 on satiety, fat oxidation, energy expenditure and body weight. International Journal of Obesity $7 \quad 25: 1087-1094$.

Koziol, M. J. (1992). Chemical composition and nutritional evaluation of quinoa (Chenopodium 9 quinoa willd.). Journal of Food Composition and Analysis 5: 35-68.

Kriketos, A. D., Thompson, H. R., Greene, H. and Hill, J. O. (1999). (-)-hydroxycitric acid does not affect energy expenditure and substrate oxidation in adult males in a post-absorptive state. International Journal of Obesity 23: 867-873.

13 Kumari, K. and Augusti, K. T. (2007). Lipid lowering effect of s-methyl cysteine sulfoxide from allium cepa linn in high cholesterol diet fed rats. Journal of Ethnopharmacology 109: 367-371.

Kumari, K., Mathew, B. C. and Augusti, K. T. (1995). Antidiabetic and hypolipidemic effects of smethyl cysteine sulfoxide isolated from Allium cepa Linn. Indian Journal of Biochemistry and Biophysics 32: 49-54.

Kurtz, T., Morris, R. and Pershadsingh, H. (1989). The Zucker fatty rat as a genetic model of obesity and hypertension. Hypertension 13: 896-901.

Kuzu, N., Bahcecioglu, I. H., Metin, K., Ozercan, I. H., Tuzcu, M., Yalniz, M., Ustundag, B. and Sahin, K. (2007). Role of melatonin in treatment of nonalcoholic steatohepatitis in rats induced bu high fat diet. Journal of Hepatology 46: S263.

Kwon, D. Y., Jung, Y. S., Kim, S. J., Park, H. K., Park, J. H. and Kim, Y. C. (2009a). Impaired sulfur-amino acid metabolism and oxidative stress in nonalcoholic fatty liver are alleviated by betaine supplementation in Rats. Journal of Nutrition 139: 63-68. 
1 Kwon, D. Y., Kim, Y. S., Hong, S. M. and Park, S. (2009b). Long-term consumption of saponins 2 derived from Platycodi radix (22 years old) enhances hepatic insulin sensitivity and glucose3 stimulated insulin secretion in $90 \%$ pancreatectomized diabetic rats fed a high-fat diet. British $4 \quad$ Journal of Nutrition 101: 358-366.

5 Labadie, P. (1974). Labile Methyls and Lipotropic Effect. Revue du Praticien 24: 4839-4854.

6 Ladyman, J. A. R., Hitz, W. D. and Hanson, A. D. (1980). Translocation and metabolism of glycine 7 betaine by barley plants in relation to water-stress. Planta 150: 191-196.

8 Laird, R. D. and Drill, V. A. (1971). Lipotropic activity of inositol and chlortetracycline alone and 9 in various combinations of choline, vitamin B12 and folic acid - Activity of 3 liver extracts with 10 assays for these substances. Archives Internationales de Pharmacodynamie et de Thérapie 194: $11 \quad 103-116$.

12 Laird, R. D., McCormick, H. M. and Drill, V. A. (1965). Lipotropic activity of combinations of 13 choline, vitamins $\mathrm{B}_{12}$ and $\mathrm{B}_{12 \mathrm{~b}}$, folic acid, and citrovorum factor. Toxicology and Applied 14 Pharmacology 7: 247-256.

15 Laraki, L., Pelletier, X., Mourot, J. and Debry, G. (1993). Effects of dietary phytosterols on liver 16 lipids and lipid metabolism enzymes. Annals of Nutrition and Metabolism 37: 129-133.

17 Larsson, S. C., Giovannucci, E., Bergkvist, L. and Wolk, A. (2005). Whole grain consumption and 18 risk of colorectal cancer: a population-based cohort of 60000 women. British Journal of Cancer 19 92: 1803-1807.

20 Lawrence, S. P. (1993). Transient focal hepatic defects related to sustained-selease niacin. Journal 21 of Clinical Gastroenterology 16: 234-236.

22 Leclerc, J. and Miller, M. L. (1989). Inositol and choline levels in the diet and neutral lipid hepatic 23 content of lactating rat. International Journal for Vitamin and Nutrition Research 59: 180-183.

24 Lee, S., Gura, K. M. and Puder, M. (2007a). Omega-3 fatty acids and liver disease. Hepatology 45 : $25 \quad 841-845$. 
1 Lee, S. H., Park, H. J., Cho, S. Y., Jung, H. J., Cho, S. M., Cho, Y. S. and Lillehoj, H. S. (2005).

2 Effects of dietary phytic acid on serum and hepatic lipid levels in diabetic KK mice. Nutrition $3 \quad$ Research 25: 869-876.

4 Lee, S.-H., Park, H.-J., Chun, H.-K., Cho, S.-Y., Jung, H.-J., Cho, S.-M., Kim, D.-Y., Kang, M.-S. 5 and Lillehoj, H. S. (2007b). Dietary phytic acid improves serum and hepatic lipid levels in aged $6 \quad$ ICR mice fed a high-cholesterol diet. Nutrition Research 27: 505-510.

7 Lee, Y. H. and Yeh, Y. Y. (2003). Inhibitory effects of garlic extract and water-soluble organosulfur compounds of garlic on cholestergenesis in HepG-2 cells. FASEB Journal 17: A752-A752.

Lennon, D. L., Stratman, F. W., Shrago, E., Nagle, F. J., Madden, M., Hanson, P. and Carter, A. L. 10 (1983). Effects of acute moderate-intensity exercise on carnitine metabolism in men and women. 11 Journal of Applied Physiology 55: 489-495.

12 Leon, J., Acuña-Castroviejo, D., Sainz, R. M., Mayo, J. C., Tan, D.-X. and Reiter, R. J. (2004). 13 Melatonin and mitochondrial function. Life Sciences 75: 765-790.

14 Leonhardt, M. and Langhans, W. (2002). Hydroxycitrate has long-term effects on feeding behavior, 15 body weight regain and metabolism after body weight loss in male rats. Journal of Nutrition 132: $16 \quad 1977-1982$.

17 Lettéron, P., Duchatelle, V., Berson, A., Fromenty, B., Fisch, C., Degott, C., Benhamou, J. P. and 18 Pessayre, D. (1993). Increased ethane exhalation, an in vivo index of lipid peroxidation, in 19 alcohol-abusers. Gut 34: 409-414.

20 Lewis, Y. S. and Neelakantan, S. (1965). (-)-Hydroxycitric acid - the principal acid in the fruits of 21 Garcinia cambogia desr. Phytochemistry 4: 619-625.

22 Li, Z., Agellon, L. B. and Vance, D. E. (2005). Phosphatidylcholine homeostasis and liver failure. 23 Journal of Biological Chemistry 280: 37798-37802.

24 Li, Z. and Vance, D. E. (2008). Phosphatidylcholine and choline homeostasis. Journal of Lipid $25 \quad$ Research 49: 1187-1194. 
1 Liang, L. J., Yin, X. Y., Luo, S. M., Zheng, J. F., Lu, M. D. and Huang, J. F. (1999). A study of the 2 ameliorating effects of carnitine on hepatic steatosis induced by total parenteral nutrition in rats. 3 World Journal of Gastroenterology 5: 312-315.

4 Lieber, C. S. (1997). Ethanol metabolism, cirrhosis and alcoholism. Clinica Chimica Acta 257: 5954.

6 Lim, K., Ryu, S., Ohishi, Y., Watanabe, I., Tomi, H., Suh, H., Lee, W. K. and Kwon, T. (2002). 7 Short-term (-)-hydroxycitrate ingestion increases fat oxidation during exercise in athletes. Journal 8

9 Lin, C.-C. and Yin, M.-C. (2008). Effects of cysteine-containing compounds on biosynthesis of 10 triacylglycerol and cholesterol and anti-oxidative protection in liver from mice consuming a high11 fat diet. British Journal of Nutrition 99: 37-43.

12 Lin, C.-C., Yin, M.-C., Hsu, C.-C. and Lin, M.-P. (2004). Effect of five cysteine-containing 13 compounds on three lipogenic enzymes in Balb/cA mice consuming a high saturated fat diet. $14 \quad$ Lipids 39: 843-848.

15 Lin, C.-C., Yin, M.-C. and Liu, W.-H. (2008). Alleviative effects of s-allyl cysteine and s-ethyl 16 cysteine on MCD diet-induced hepatotoxicity in mice. Food and Chemical Toxicology 46: 3401173406.

18 Lin, M., Kao, S., Chung, P., Chan, K., Yang, M. and Wang, C. (2009). Improvement for high fat 19 diet-induced hepatic injuries and oxidative stress by flavonoid-enriched extract from Nelumbo 20 nucifera leaf. Journal of Agricultural and Food Chemistry 57: 5925-5932.

21 Lipmann, F., Kaplan, N. O., Novelli, G. D., Tuttle, L. C. and Guirard, B. M. (1947). Coenzyme for 22 acetylation, a pantothenic acid derivative. Journal of Biological Chemistry 167: 869-870.

23 Liu, L. and Yeh, Y.-Y. (2000). Inhibition of cholesterol biosynthesis by organosulfur compounds 24 derived from garlic. Lipids 35: 197-203.

25 Liu, L., Zubik, L., Collins, F. W., Marko, M. and Meydani, M. (2004). The antiatherogenic 26 potential of oat phenolic compounds. Atherosclerosis 175: 39-49. 
1 Locker, J., Reddy, T. V. and Lombardi, B. (1986). DNA methylation and hepatocarcinogenesis in 2 rats fed a choline-devoid diet. Carcinogenesis 7: 1309-1312.

3 Lombardi, B. (1971). Effects of choline deficiency on rat hepatocytes. Federation Proceedings 30: $4 \quad 139-142$.

5 Lombardi, B., Pani, P. and Schlunk, F. F. (1968). Choline-deficiency fatty liver: impaired release of 6 hepatic triglycerides. Journal of Lipid Research 9: 437-446.

7 Lopez, H. W., Leenhardt, F., Coudray, C. and Remesy, C. (2002). Minerals and phytic acid 8 interactions: is it a real problem for human nutrition? International Journal of Food Science and 9 Technology 37: 727-739.

10 Lopez, H. W., Vallery, F., Levrat-Verny, M. A., Coudray, C., Demigne, C. and Remesy, C. (2000). 11 Dietary phytic acid and wheat bran enhance mucosal phytase activity in rat small intestine. 12 Journal of Nutrition 130: 2020-2025.

13 Lotito, S. B. and Frei, B. (2006). Consumption of flavonoid-rich foods and increased plasma 14 antioxidant capacity in humans: Cause, consequence, or epiphenomenon? Free Radical Biology $15 \quad$ and Medicine 41: 1727-1746.

16 Lott, J. N. A., Ockenden, I., Raboy, V. and Batten, G. D. (2000). Phytic acid and phosphorus in 17 crop seeds and fruits: a global estimate. Seed Science Research 10: 11-33.

18 Lowenstein, J. M. (1971). Effect of (-)-hydroxycitrate on fatty acid synthesis by rat liver in vivo. 19 Journal of Biological Chemistry 246: 629-632.

20 Luo, Q.-F., Sun, L., Si, J.-Y. and Chen, D.-H. (2008). Hypocholesterolemic effect of stilbenes 21 containing extract-fraction from Cajanus cajan L. on diet-induced hypercholesterolemia in mice. $22 \quad$ Phytomedicine 15: 932-939.

23 Madani, S., Lopez, S., Blond, J. P., Prost, J. and Belleville, J. (1998). Highly purified soybean 24 protein is not hypocholesterolemic in rats but stimulates cholesterol synthesis and excretion and 25 reduces polyunsaturated fatty acid biosynthesis. Journal of Nutrition 128: 1084-1091. 
1 Mahboob, S. (1975). Effect of pantothenic-acid deficiency on microsomal lipids of rat-liver. Annals 2 of Nutrition and Metabolism 19: 91-95.

3 Mahboob, S. and Estes, L. W. (1978). Effect of pantothenic-acid deficiency on rat hepatocytes. 4 Annals of Nutrition and Metabolism 22: 177-180.

5 Maiese, K., Morhan, S. D. and Chong, Z. Z. (2007). Oxidative stress biology and cell injury during $6 \quad$ type 1 and type 2 diabetes Mellitus. Current Neurovascular Research 4: 63-71.

7 Mamone, F., Riggio, S., Mandraffino, G., Maimone, S., La Scala, M., Sardo, M. A. and Saitta, A. 8 (2009). Assessment of liver stiffness values in subjects affected by familial combined 9 hyperlipidemia with different degree of hepatic steatosis. Nutrition, Metabolism and 10 Cardiovascular Diseases 19: S15.

11 Mannarino, M. R., Pirro, M., Helou, R., Covelli, D., Pucci, G., Schillaci, G. and Mannarino, E. 12 (2009). Liver steatosis: an independent predictor of arterial stiffness in dyslipidemic patients. 13 Nutrition, Metabolism and Cardiovascular Diseases 19: S15-S16.

14 Mantovani, M. S., Bellini, M. F., Angeli, J. P. F., Oliveira, R. J., Silva, A. F. and Ribeiro, L. R. 15 (2008). Beta-glucans in promoting health: Prevention against mutation and cancer. Mutation 16 Research-Reviews in Mutation Research 658: 154-161.

17 Marchesini, G., Brizi, M., Morselli-Labate, A. M., Bianchi, G., Bugianesi, E., McCullough, A. J., 18 Forlani, G. and Melchionda, N. (1999). Association of nonalcoholic fatty liver disease with 19 insulin resistance. American Journal of Medicine 107: 450-455.

20 Martinez, L. O., Jacquet, S., Esteve, J.-P., Rolland, C., Cabezon, E., Champagne, E., Pineau, T., 21 Georgeaud, V., Walker, J. E., Terce, F., Collet, X., Perret, B. and Barbaras, R. (2003). Ectopic 22 [beta]-chain of ATP synthase is an apolipoprotein A-I receptor in hepatic HDL endocytosis. 421: $23 \quad$ Nature 75-79.

24 Masuda, T., Kawano, A., Kitahara, K., Nagashima, K., Aikawa, Y. and Arai, S. (2003). 25 Quantitative determination of sugars and myo-inositol in citrus fruits grown in Japan using high- 
1 performance anion-exchange chromatography. Journal of Nutritional Science and Vitaminology 49: 64-68.

3 Mayor, F., Veloso, D. and Williams.Dh (1967). Effects of nicotinic acid on acetoacetate and 34 hydroxybutyrate concentrations of rat blood and liver. Biochemical Journal 104: 57a-57P.

McCance, R. A. and Widdowson, E. M. (1935). Phytin in human nutrition. Biochemical Journal 29:

$6 \quad 2694-2699$.

7 McCarty, M. F. (1994). Promotion of hepatic lipid oxidation and gluconeogenesis as a strategy for appetite control. Medical Hypotheses 42: 215-225.

9 McCarty, M. F. (2000). Co-administration of equimolar doses of betaine may alleviate the 10 hepatotoxic risk associated with niacin therapy. Medical Hypotheses 55: 189-194.

11 McCarty, M. F. (2002). Policosanol safely down-regulates HMG-CoA reductase - potential as a 12 component of the Esselstyn regimen. Medical Hypotheses 59: 268-279.

13 McHenry, E. W. and Gavin, G. (1941). The B vitamins and fat metabolism. IV. The synthesis of fat 14 from protein. Journal of Biological Chemistry 138: 471-475.

15 McHenry, E. W. and Patterson, J. M. (1944). Lipotropic factors. Physiological reviews 24: 128-167. 16 McKenney, J. M., Proctor, J. D., Harris, S. and Chinchili, V. M. (1994). A comparison of the 17 efficacy and toxic effects of sustained- vs immediate-release niacin in hypercholesterolemic 18 patients. Journal of the American Medical Association 271: 672-677.

19 Mellen, P. B., Walsh, T. F. and Herrington, D. M. (2008). Whole grain intake and cardiovascular 20 disease: A meta-analysis. Nutrition Metabolism and Cardiovascular Diseases 18: 283-290.

21 Menendez, R., Amor, A. M., Gonzalez, R. M., Fraga, V. and Mas, R. (1996). Effect of policosanol 22 on the hepatic cholesterol biosynthesis of normocholesterolemic rats. Biological Research 29 : $23 \quad 253-257$.

24 Menendez, R., Arruzazabala, L., Mas, R., DelRio, A., Amor, A. M., Gonzalez, R. M., Carbajal, D., 25 Fraga, V., Molina, V. and Illnait, J. (1997). Cholesterol-lowering effect of policosanol on rabbits 
$1 \quad$ with hypercholesterolaemia induced by a wheat starch-casein diet. British Journal of Nutrition 77: $2923-932$.

3 Menendez, R., Fernandez, S. I., Del Rio, A., Gonzalez, R. M., Fraga, V., Amor, A. M. and Mas, R. 4 M. (1994). Policosanol inhibits cholesterol biosynthesis and enhances low density lipoprotein $5 \quad$ processing in cultured human fibroblasts. Biological Research 27.

6 Mercier, J., Lumbroso, A., Vovan, L. and Dessaigne, S. (1967). On the hepatoprotective lipotropic 7 proprieties of hexanicit. Thérapie 22: 1153-1158.

8 Merrill, J. M. and Lemley-Stone, J. (1957). Effects of nicotinic acid on serum and tissue cholesterol $9 \quad$ in rabbits. Circulation Research 5: 617-619.

10 Miglio, F., Rovati, L. C., Santoro, A. and Setnikar, I. (2000). Efficacy and safety of oral betaine 11 glucuronate in non-alcoholic steatohepatitis - A double-blind, randomized, parallel-group, 12 placebo-controlled prospective clinical study. Arzneimittel Forschung-Drug Research 50: 722 13727.

14 Miller, O. N., Hamilton, J. G. and Goldsmith, G. A. (1960). Investigation of the Mechanism of 15 Action of Nicotinic Acid on Serum Lipid Levels in Man. American Journal of Clinical Nutrition 16 8: $480-490$.

17 Minhajuddin, M., Beg, Z. H. and Iqbal, J. (2005). Hypolipidemic and antioxidant properties of 18 tocotrienol rich fraction isolated from rice bran oil in experimentally induced hyperlipidemic rats. 19 Food and Chemical Toxicology 43: 747-753.

20 Mizrahi, A., Knekt, P., Montonen, J., Laaksonen, M. A., Heli?vaara, M. and J?rvinen, R. (2009). 21 Plant foods and the risk of cerebrovascular diseases: a potential protection of fruit consumption. 22 British Journal of Nutrition 102: 1075-1083.

23 Moat, S. J., Hill, M. H., McDowell, I. F. W., Pullin, C. H., Ashfield-Watt, P. A. L., Clark, Z. E., 24 Whiting, J. M., Newcombe, R. G., Lewis, M. J. and Powers, H. J. (2003). Reduction in plasma 25 total homocysteine through increasing folate intake in healthy individuals is not associated with 
1 changes in measures of antioxidant activity or oxidant damage. European Journal of Clinical $2 \quad$ Nutrition 57: 483-489.

3 Moghadasian, M. H., Nguyen, L. B., Shefer, S., Salen, G., Batta, A. K. and Frohlich, J. J. (2001).

$4 \quad$ Hepatic cholesterol and bile acid synthesis, low-density lipoprotein receptor function, and plasma 5 and fecal sterol levels in mice: effects of apolipoprotein E deficiency and probucol or phytosterol $6 \quad$ treatment. Metabolism-Clinical and Experimental 50: 708-714.

7 Moiseenok, A. G., Sheibak, V. M. and Gurinovich, V. A. (1987). Hepatic CoA, S-Acyl-CoA, 8 biosynthetic precursors of the coenzyme and pantothenate-protein complexes in dietary 9 pantothenic-acid deficiency. International Journal for Vitamin and Nutrition Research 57: 71-77.

10 Mongeau, R. and Brassard, R. (1982). Insoluble dietary fiber from breakfast cereals and brans: bile 11 salt binding and water-holding capacity in relation to particle size. Cereal Chemistry 59: 413-417.

12 Mookerjea, S. (1971). Action of choline in lipoprotein metabolism. Federation Proceedings 30: $13 \quad 143-150$.

14 Mookerjea, S., Park, C. and Kuksis, A. (1975). Lipid profiles of plasma lipoproteins of fasted and 15 fed normal and choline-deficient rats. Lipids 10: 374-382.

16 Moon, Y. S., Keller, W. L. and Park, C. S. (1998). Dietary lipotrope-mediated mammary 17 carcinogenesis in female rats. Nutrition Research 18: 1605-1614.

18 Moon, Y. S., Latasa, M. J., Griffin, M. J. and Sul, H. S. (2002). Suppression of fatty acid synthase 19 promoter by polyunsaturated fatty acids. Journal of Lipid Research 43: 691-698.

20 Morgan, A. F. and Lewis, E. M. (1953). The modification of choline deficiency by simultaneous 21 pantothenic acid deficiency. Journal of Biological Chemistry 200: 839-850.

22 Morin, R. J. (1967). The influence of cholesterol on incorporation of $\left(\mathrm{I}-{ }^{14} \mathrm{C}\right)$ acetate into 23 phosphatidyl choline and phosphatidyl ethanolamine of rat-liver Mitochondria. Biochimica et 24 Biophysica Acta 144: 594-604. 
1 Morise, A., Thomas, C., Landrier, J.-F., Besnard, P. and Hermier, D. (2009). Hepatic lipid 2 metabolism response to dietary fatty acids is differently modulated by PPAR $\alpha$ in male and female 3 mice. European Journal of Nutrition 48: 465-473.

4 Moskaug, J. O., Carlsen, H., Myhrstad, M. C. and Blomhoff, R. (2005). Polyphenols and 5 glutathione synthesis regulation. American Journal of Clinical Nutrition 81: 277S-283S.

6 Murakami, A., Takahashi, D., Koshimizu, K. and Ohigashi, H. (2003). Synergistic suppression of 7 superoxide and nitric oxide generation from inflammatory cells by combined food factors. Mutation Research-Fundamental and Molecular Mechanisms of Mutagenesis 523-524: 151-161.

9 Murtaugh, M. A., Jacobs, D. R., Jacob, B., Steffen, L. M. and Marquart, L. (2007). Epidemiological 10 support for the protection of whole grains against diabetes. Proceedings of the Nutrition Society $11 \quad 62: 143-149$.

12 Nadeau, G., Rouleau, Y. and Delage, J. (1954). The fate of the liver in alcoholics. II. Use of 13 lipotropic factors in the treatment of hepatic dysfunction. Laval Med 19: 52-58.

14 Nadler, J., Buchanan, T., Natarajan, R., Antonipillai, I., Bergman, R. and Rude, R. (1993). 15 Magnesium deficiency produces insulin resistance and increased thromboxane synthesis. 16 Hypertension 21: 1024-1029.

17 Nagata, Y., Ishiwaki, N. and Sugano, M. (1982). Studies on the mechanism of 18 antihypercholesterolemic action of soy protein and soy protein-type amino acid mixtures in 19 relation to the casein counterparts in rats. Journal of Nutrition 112: 1614-1625.

20 Nageswara Rao, R. and Sakariah, K. K. (1988). Lipid-lowering and antiobesity effect of (21 )hydroxycitric acid. Nutrition Research 8: 209-212.

22 Nagiel-Ostaszewski, I. and Lau-Cam, C. A. (1990). Protection by pantethine, pantothenic-acid and 23 cystamine against carbon tetrachloride-induced hepatotoxicity in the rat. Research 24 Communications in Chemical Pathology and Pharmacology 67: 289-292.

25 Nagura, J., Iso, H., Watanabe, Y., Maruyama, K., Date, C., Toyoshima, H., Yamamoto, A., 26 Kikuchi, S., Koizumi, A., Kondo, T., Wada, Y., Inaba, Y., Tamakoshi, A. and null, t. J. S. g. 
1 (2009). Fruit, vegetable and bean intake and mortality from cardiovascular disease among 2 Japanese men and women: the JACC Study. British Journal of Nutrition 102: 285-292.

3 Nakamura, Y., Kaihara, A., Yoshii, K., Tsumura, Y., Ishimitsu, S. and Tonogai, Y. (2001). Effects

4 of the oral administration of green tea polyphenol and tannic acid on serum and hepatic lipid $5 \quad$ contents and fecal steroid excretion in rats. Journal of Health Science 47: 107-117.

6 Nakamura, Y. and Tonogai, Y. (2002). Effects of grape seed polyphenols on serum and hepatic

7 lipid contents and fecal steroid excretion in normal and hypercholesterolemic rats. Journal of Health Science 48: 570-578.

9 Naruta, E. and Buko, V. (2001). Hypolipidemic effect of pantothenic acid derivatives in mice with 10 hypothalamic obesity induced by aurothioglucose. Experimental and Toxicologic Pathology 53 : $11 \quad 393-398$.

12 Nauss, K. M., Connor, A. M., Kavanaugh, A. and Newberne, P. M. (1982). Alterations in immune 13 function in rats caused by dietary lipotrope deficiency - effect of age. Journal of Nutrition 112: $14 \quad 2333-2341$.

15 Navarranne, Fritz, Tanguy, Meriaux and Marion (1964). First biological results of a new lipotropic 16 formula. La Clinique (Paris) 59: 443-445.

17 Nehra, V., Angulo, P., Buchman, A. L. and Lindor, K. D. (2001). Nutritional and metabolic 18 considerations in the etiology of nonalcoholic steatohepatitis. Digestive Diseases and Sciences 46: $19 \quad 2347-2352$

20 Neuschwander-Tetri, B. A. and Caldwell, S. H. (2003). Nonalcoholic steatohepatitis: Summary of 21 an AASLD Single Topic Conference. Hepatology 37: 1202-1219.

22 Neveu, V., Perez-Jimenez, J., Vos, F., Crespy, V., du Chaffaut, L., Mennen, L., Knox, C., Eisner, 23 R., Cruz, J., Wishart, D. and Scalbert, A. (2010). Phenol-explorer: an online comprehensive 24 database on polyphenol contents in foods. Database 2010: bap024-.

25 Newberne, P. M. and Rogers, A. E. (1986). Labile methyl groups and the promotion of cancer. 26 Annual Review of Nutrition 6: 407-432. 
1 Newberne, P. M., Wilson, R. and Rogers, A. E. (1971). Effects of a low-lipotrope diet on response 2 of young male rats to pyrrolizidine alkaloid, monocrotaline. Toxicology and Applied $3 \quad$ Pharmacology 18: 387-397.

4 Newby, P. (2009). Plant foods and plant-based diets: protective against childhood obesity? $5 \quad$ American Journal of Clinical Nutrition 89: 1572S-1587.

6 Nichols, M. R. and Morimoto, B. H. (1999). Tyrosine kinase-independent inhibition of cyclic-AMP 7 phosphodiesterase by genistein and tyrphostin 51. Archives of Biochemistry and Biophysics $\mathbf{3 6 6}$ : $8224-230$.

9 Nichols, M. R. and Morimoto, B. H. (2000). Differential inhibition of multiple cAMP 10 phosphodiesterase isozymes by isoflavones and tyrphostins. Molecular Pharmacology 57: 73811745.

12 Nieminen, P., Kakela, R., Mustonen, A. M., Hyvarinen, H. and Asikainen, J. (2001). Exogenous 13 melatonin affects lipids and enzyme activities in mink (Mustela vison) liver. Comparative 14 Biochemistry and Physiology C-Toxicology and Pharmacology 128: 203-211.

15 Nieminen, P., Mustonen, A. M., Karja, V., Asikainen, J. and Rouvinen-Watt, K. (2009). Fatty acid 16 composition and development of hepatic lipidosis during food deprivation-mustelids as a potential 17 Animal model for liver steatosis. Experimental Biology and Medicine 234: 278-286.

18 Noethlings, U., Schulze, M. B., Weikert, C., Boeing, H., van der Schouw, Y. T., Bamia, C., 19 Benetou, V., Lagiou, P., Krogh, V., Beulens, J. W. J., Peeters, P. H. M., Halkjaer, J., Tjonneland, 20 A., Tumino, R., Panico, S., Masala, G., Clavel-Chapelon, F., de Lauzon, B., Boutron-Ruault, M.21 C., Vercambre, M.-N., Kaaks, R., Linseisen, J., Overvad, K., Arriola, L., Ardanaz, E., Gonzalez, 22 C. A., Tormo, M.-J., Bingham, S., Khaw, K.-T., Key, T. J. A., Vineis, P., Riboli, E., Ferrari, P., 23 Boffetta, P., Bueno-de-Mesquita, H. B., van der A, D. L., Berglund, G., Wirfalt, E., Hallmans, G., 24 Johansson, I., Lund, E. and Trichopoulo, A. (2008). Intake of vegetables, legumes, and fruit, and 25 risk for all-cause, cardiovascular, and cancer mortality in a European diabetic population. Journal 26 of Nutrition 138: 775-781. 
1 Nolte, K. D., Hanson, A. D. and Gage, D. A. (1997). Proline accumulation and methylation to

2 proline betaine in citrus: Implications for genetic engineering of stress resistance. Journal of 3 American Society for Horticultural science 122: 8-13.

4 Novelli, G. D., Kaplan, N. O. and Lipmann, F. (1949). The liberation of pantothenic acid from coenzyme A. Journal of Biological Chemistry 177: 97-107.

6 Nunn, S. L., Tauxe, W. N. and Juergens, J. L. (1961). Effect of nicotinic acid on human cholesterol 7 biosynthesis. Circulation 24: 1099.

8 Oakenfull, D. G., Fenwick, D. E., Hood, R. L., Topping, D. L., Illman, R. L. and Storer, G. B. 9 (1979). Effects of saponins on bile-acids and plasma-lipids in the rat. British Journal of Nutrition 10 42: $209-216$

11 Ohigashi, H. and Murakami, A. (2004). Cancer prevention with food factors: alone and in 12 combination. Biofactors 22: 49-55.

13 Okada, M. and Ochi, A. (1971). The effect of dietary protein level on transaminase activities and fat 14 deposition in vitamin B6-depleted rat liver. Journal of Biochemistry 70: 581-585.

15 Okada, M. and Suzuki, K. (1974). Amino acid metabolism in rats fed a high protein diet without 16 pyridoxine. Journal of Nutrition 104: 287-293.

17 Okazaki, Y. and Katayama, T. (2008). Dietary myositol hexakisphosphate, but not myo-inositol, 18 clearly improves hypercholesterolemia in rats fed casein-type amino acid mixtures and 1,1,119 trichloro-2,2-bis (p-chlorophenyl) ethane. Nutrition Research 28: 714-721.

20 Okazaki, Y., Kayashima, T. and Katayama, T. (2003). Effect of dietary phytic acid on hepatic 21 activities of lipogenic and drug-metabolizing enzymes in rats fed 1,1,1-trichloro-2,2-bis (P22 chlorophenyl) ethane (DDT). Nutrition Research 23: 1089-1096.

23 Okazaki, Y., Setoguchi, T. and Katayama, T. (2006). Effects of dietary myo-inositol, D-chiro24 inositol and L-chiro-inositol on hepatic lipids with reference to the hepatic myo-inositol status in 25 rats fed on 1,1,1-trichloro-2,2-bis (p-chlorophenyl) ethane. Bioscience, Biotechnology, and 26 Biochemistry 70: 2766-2770. 
1 Olson, R. E., Jablonski, J. R. and Taylor, E. (1958a). The Effect of Dietary Protein, Fat, and

2 Choline upon the Serum Lipids and Lipoproteins of the Rat. American Journal of Clinical $3 \quad$ Nutrition 6: 111-118.

4 Olson, R. E., Vester, J. W., Gursey, D., Davis, N. and Longman, D. (1958b). The effect of low5 protein diets upon serum cholesterol in man. American Journal of Clinical Nutrition 6: 310-324.

6 Olthof, M. R. and Verhoef, P. (2005). Effects of betaine intake on plasma homocysteine 7 concentrations and consequences for health. Current Drug Metabolism 6: 15-22.

8 Onning, G. and Asp, N. G. (1995). Effect of oat saponins on plasma and liver lipids in gerbils 9 (Meriones unguiculatus) and rats. British Journal of Nutrition 73: 275-286.

10 Onomi, S., Okazaki, Y. and Katayama, T. (2004). Effect of dietary level of phytic acid on hepatic 11 and serum lipid status in rats fed a high-sucrose diet. Bioscience, Biotechnology and Biochemistry 12 68: $1379-1381$.

13 Orbetsova, V. T. (1977). Changes in levels of serum and liver lipids after a single injection of 14 nicotinic-acid. Dokladi Na Bolgarskata Akademiya Na Naukite 30: 613-616.

15 Orbetsova, V. T., Kiprov, D. I. and Vucheva, N. V. (1977). Effect of nicotinic-acid on level of 16 serum and liver lipids in spontaneously hypertensive rats fed an atherogenic diet. Dokladi $\mathrm{Na}$ 17 Bolgarskata Akademiya Na Naukite 30: 465-468.

18 Orbetsova, V. T., Polyakova, E. D., Klimova, T. A. and Dizhe, E. B. (1976). Influence of nicotinic19 acid on the synthesis of cholesterol and fatty-acids in liver of albino-rats. Dokladi Na Bolgarskata $20 \quad$ Akademiya Na Naukite 29: 137-140.

21 Ortega, M. F. (1989). Effect of dietary lysine level and protein restriction on the lipids and carnitine 22 levels in the liver of pregnant rats. Annals of Nutrition and Metabolism 33: 162-169.

23 Osumi, Y., Nagasaka, Y. and Shimamot.K (1969). Lipid metabolism in rats with fatty liver caused 24 by low protein diet and effects of oral administration of L-methionine L-cysteine pantethine and 25 calcium pantothenate upon it. Japanese Journal of Pharmacology 19: 74-88.

26 Ournac, A. (1970). Vitamins of wine. Annales de la Nutrition et de l'Alimentation 24: B333-B365. 
1 Owens (1942). The comparative effects of inositol and lipocaic in depancreatized dogs. Federation

$2 \quad$ Proceedings 1: 65.

3 Pagano, G., Pacini, G., Musso, G., Gambino, R., Mecca, F., Depetris, N., Cassader, M., David, E.,

4 Cavallo-Perin, P. and Rizzetto, M. (2002). Nonalcoholic steatohepatitis, insulin resistance, and

5 metabolic syndrome: Further evidence for an etiologic association. Hepatology 35: 367-372.

6 Pan, M., Song, Y.-L., Xu, J.-M. and Gan, H.-Z. (2006). Melatonin ameliorates nonalcoholic fatty $7 \quad$ liver induced by high-fat diet in rats. Journal of Pineal Research 41: 79-84.

8 Pardue, W. O. (1961). Severe liver dysfunction during nicotinic acid therapy. Journal of the $9 \quad$ American Medical Association 175: 137-138.

10 Parker, R. A., Pearce, B. C., Clark, R. W., Gordon, D. A. and Wright, J. J. (1993). Tocotrienols 11 regulate cholesterol production in mammalian cells by post-transcriptional suppression of 312 hydroxy-3-methylglutaryl-coenzyme A reductase. Journal of Biological Chemistry 268: 112301311238.

14 Parker, T. L., Miller, S. A., Myers, L. E., Miguez, F. E. and Engeseth, N. J. (2010). Evaluation of 15 synergistic antioxidant potential of complex mixtures using oxygen radical absorbance capacity 16 (ORAC) and electron paramagnetic resonance (EPR). Journal of Agricultural and Food 17 Chemistry 58: 209-217.

18 Parsons, W. B. (1961a). Studies of nicotinic acid use in hypercholesteremia: changes in hepatic 19 function, carbohydrate tolerance, and uric acid metabolism. Archives of Internal Medicine 107: $20 \quad 85-99$.

21 Parsons, W. B., Jr. and Flinn, J. H. (1959). Reduction of serum cholesterol levels and beta22 lipoprotein cholesterol levels by nicotinic acid. Archives of Internal Medicine 103: 123-130.

23 Parsons, W. B. J. (1961b). Reduction in hepatic synthesis of cholesterol from $\mathrm{C}^{14}$-acetate in 24 hypercholesterolemic patients by nicotinic acid. Circulation 24: 1099-1100. 
1 Patrick, L. (2002). Nonalcoholic fatty liver disease: relationship to insulin sensitivity and oxidative

2 stress. Treatment approaches using vitamin E, magnesium, and betaine. Alternative Medicine 3 Review 7: 276-291.

4 Pavia, M., Pileggi, C., Nobile, C. G. A. and Angelillo, I. F. (2006). Association between fruit and 5 vegetable consumption and oral cancer: a meta-analysis of observational studies. American $6 \quad$ Journal of Clinical Nutrition 83: 1126-1134.

7 Pellegrini, N., Serafini, M., Colombi, B., Del Rio, D., Salvatore, S., Bianchi, M. and Brighenti, F. 8 (2003). Total antioxidant capacity of plant foods, beverages and oils consumed in Italy assessed 9 by three different in vitro assays. Journal of Nutrition 133: 2812-2819.

10 Peluso, M. R. (2006). Flavonoids attenuate cardiovascular disease, inhibit phosphodiesterase, and 11 modulate lipid homeostasis in adipose tissue and liver. Experimental Biology and Medicine 231: $12 \quad 1287-1299$.

13 Perlman, I. and Chaikoff, I. L. (1939). Radioactive phosphorus as an indicator of phospholipid 14 metabolism. Journal of Biological Chemistry 130: 593-600.

15 Perlzweig, W. A., Bernheim, M. L. C. and Bernheim, F. (1943). The methylation of nicotinamide 16 by rat liver in vitro. Journal of Biological Chemistry 150: 401-406.

17 Perrault, M. and Dormard, Y. (1966). Lipotropic effect of betaine aspartate on experimental hepatic 18 steatosis. Study using triolein-C $C^{14}$. Thérapie 21: 719-731.

19 Perry, W. F. (1960). Effect of nicotinic acid and nicotinamide on incorporation of acetate into 20 cholesterol, fatty acids and $\mathrm{CO}_{2}$ by rat liver slices. Metabolism-Clinical and Experimental 9: 68621689.

22 Pettinelli, P., del Pozo, T., Araya, J., Rodrigo, R., Araya, A. V., Smok, G., Csendes, A., Gutierrez, 23 L., Rojas, J., Korn, O., Maluenda, F., Diaz, J. C., Rencoret, G., Braghetto, I., Castillo, J., 24 Poniachik, J. and Videla, L. A. (2009). Enhancement in liver SREBP-1c/PPAR-alpha ratio and 25 steatosis in obese patients: correlations with insulin resistance and n-3 long-chain polyunsaturated 26 fatty acid depletion. Biochimica et Biophysica Acta-Molecular Basis of Disease 1792: 1080-1086. 
1 Pfiffner, J. J. and Bird, O. D. (1956). Water-soluble vitamins, Part I. Annual Review of Biochemistry 2 25: $397-434$

3 Pilvi, T. K., Seppanen-Laakso, T., Simolin, H., Finckenberg, P., Huotari, A., Herzig, K. H., 4 Korpela, R., Oresic, M. and Mervaala, E. M. (2008). Metabolomic changes in fatty liver can be 5 modified by dietary protein and calcium during energy restriction. World Journal of $6 \quad$ Gastroenterology 14: 4462-4472.

7 Poirier, L. A. and Whitehead, V. M. (1973). Folate deficiency and formiminoglutamic acid 8 excretion during chronic diethylnitrosamine administration to rats. Cancer Research 33: 383-388.

9 Popper, H. and Schaffner, F. (1952). Laboratory diagnosis of liver disease: coordinated use of 10 histological and biochemical observations. Journal of the American Medical Association 150: $11 \quad 1367-1372$.

12 Preuss, H. G., Bagchi, D., Bagchi, M., Rao, C. V. S., Dey, D. K. and Satyanarayana, S. (2004a). 13 Effects of a natural extract of (-)-hydroxycitric acid (HCA-SX) and a combination of HCA-SX 14 plus niacin-bound chromium and Gymnema sylvestre extract on weight loss. Diabetes, Obesity 15 and Metabolism 6: 171-180.

16 Preuss, H. G., Bagchi, D., Bagchi, M., Rao, C. V. S., Satyanarayana, S. and Dey, D. K. (2004b). 17 Efficacy of a novel, natural extract of (-)-hydroxycitric acid (HCA-SX) and a combination of 18 HCA-SX, niacin-bound chromium and Gymnema sylvestre extract in weight management in 19 human volunteers: A pilot study. Nutrition Research 24: 45-58.

20 Prior, R. L. (2003). Fruits and vegetables in the prevention of cellular oxidative damage. American 21 Journal of Clinical Nutrition 78: 570S-578S.

22 Quan, P. C. and Le Breton, E. (1973). Study of fatty acid composition of 3 main classes of 23 phospholipid in the liver, in rats having eaten, after weaning, during 3 or 45 days, diets containing 24 different oils. Effects of fasting and lipotropic agents. Comparison with cardiac phospholipids. 25 Comptes-Rendus Hebdomadaires des Séances de l'Académie des Sciences. Série D: Sciences $26 \quad$ Naturelles 276: 1585-1588. 
1 Qureshi, A. A., Bradlow, B. A., Salser, W. A. and Brace, L. D. (1997). Novel tocotrienols of rice 2 bran modulate cardiovascular disease risk parameters of hypercholesterolemic humans. Journal of $3 \quad$ Nutritional Biochemistry 8: 290-298.

4 Qureshi, A. A., Burger, W. C., Peterson, D. M. and Elson, C. E. (1986). The structure of an 5 inhibitor of cholesterol biosynthesis isolated from barley. Journal of Biological Chemistry 261: $6 \quad 10544-10550$.

7 Rader, J. I., Calvert, R. J. and Hathcock, J. N. (1992). Hepatic toxicity of unmodified and time8 release preparations of niacin. American Journal of Medicine 92: 77-81.

9 Rahman, I., Biswas, S. K. and Kirkham, P. A. (2006). Regulation of inflammation and redox 10 signaling by dietary polyphenols. Biochemical Pharmacology 72: 1439-1452.

11 Recknagel, R. O. (1967). Carbon tetrachloride hepatotoxicity. Pharmacological Reviews 19: 145 12208

13 Reddy, N. R., Sathe, S. K. and Salunkhe, D. K. (1982). Phytates in legumes and cereals. Advances 14 in Food Research 28: 1-92.

15 Reeves, P. G., Nielsen, F. H. and Fahey, G. C., Jr. (1993). AIN-93 purified diets for laboratory 16 rodents: final report of the American Institute of Nutrition ad hoc writing committee on the 17 reformulation of the AIN-76A rodent diet. Journal of Nutrition 123: 1939-1951.

18 Reid, A. E. (2001). Nonalcoholic steatohepatitis. Gastroenterology 121: 710-723.

19 Reimund, E. and Ramos, A. (1994). Niacin-induced hepatitis and thrombocytopenia after 10 years 20 of niacin use. Journal of Clinical Gastroenterology 18: 270-271.

21 Rejman, J. and Kozubek, A. (2003). ). Inhibitory effect of natural phenolic lipids upon nad22 dependent dehydrogenases and on triglyceride accumulation in 3T3-L1 cells in culture. Journal of 23 Agricultural and Food Chemistry 52: 246-250.

24 Rhew, T. H. and Sachan, D. S. (1986). Dose-dependent lipotropic effect of carnitine in chronic25 alcoholic rats. Journal of Nutrition 116: 2263-2269. 
1 Rikans, L. L., Arata, D. and Cederquist, D. C. (1964). Fatty livers produced in albino rats by excess

2 niacin in high fat diets: I. Alterations in enzyme and coenzyme systems induced by supplementing $340 \%$ fat diets with $0.1 \%$ of niacin. Journal of Nutrition 82: 83-87.

4 Rikans, L. L., Arata, D. and Cederquist, D. C. (1965). Fatty livers produced in albino rats by excess 5 niacin in high fat diets. II. Effect of choline supplements. Journal of Nutrition 85: 107-112.

6 Rissanen, T. H., Voutilainen, S., Virtanen, J. K., Venho, B., Vanharanta, M., Mursu, J. and Salonen, 7 J. T. (2003). Low intake of fruits, berries and vegetables is associated with excess mortality in men: the Kuopio Ischaemic Heart Disease Risk Factor (KIHD) Study. Journal of Nutrition 133: 199-204.

Rogers, A. E. (1975). Variable effects of a lipotrope-deficient, high-fat diet on chemical carcinogenesis in rats. Cancer Research 35: 2469-2474.

12 Rogers, A. E. and Newberne, P. M. (1969). Aflatoxin B1 carcinogenesis in lipotrope-deficient rats. 13 Cancer Research 29: 1965-1972.

14 Rong, N., Ausman, L. and Nicolosi, R. (1997). Oryzanol decreases cholesterol absorption and aortic 15 fatty streaks in hamsters. Lipids 32: 303-309.

16 Rosenfeld, B. (1973). Regulation by dietary choline of hepatic fatty acid synthetase in the rat. 17 Journal of Lipid Research 14: 557-562.

18 Rosolova, H., Mayer, O., Jr. and Reaven, G. (1997). Effect of variations in plasma magnesium 19 concentration on resistance to insulin-mediated glucose disposal in nondiabetic subjects. Journal 20 of Clinical Endocrinology and Metabolism 82: 3783-3785.

21 Ross, A. B., Chen, Y., Frank, J., Swanson, J. E., Parker, R. S., Kozubek, A., Lundh, T., Vessby, B., 22 Aman, P. and Kamal-Eldin, A. (2004a). Cereal alkylresorcinols elevate gamma-tocopherol levels 23 in rats and inhibit gamma-tocopherol metabolism in vitro. Journal of Nutrition 134: 506-510.

24 Ross, A. B., Kamal-Eldin, A. and Aman, P. (2004b). Dietary alkylresorcinols: absorption, 25 bioactivities, and possible use as biomarkers of whole-grain wheat- and rye-rich foods. Nutrition 26 Reviews 62: 81-95. 
1 Rubis, B., Paszel, A., Kaczmarek, M., Rudzinska, M., Jelen, H. and Rybczynska, M. (2008).

2 Beneficial or harmful influence of phytosterols on human cells? British Journal of Nutrition 100: $3 \quad 1183-1191$.

4 Rumpler, W., Seale, J., Clevidence, B., Judd, J., Wiley, E., Yamamoto, S., Komatsu, T., Sawaki, T., 5 Ishikura, Y. and Hosoda, K. (2001). Oolong tea increases metabolic rate and fat oxidation in men. $6 \quad$ Journal of Nutrition 131: 2848-2852.

7 Rushmore, T. H., Morton, M. R. and Pickett, C. B. (1991). The antioxidant responsive element. 8 Activation by oxidative stress and identification of the DNA consensus sequence required for 9 functional activity. Journal of Biological Chemistry 266: 11632-11639.

10 Russakoff, A. H. and Blumberg, H. (1944). Choline as an adjuvant to the dietary therapy of 11 cirrhosis of the liver. Annals of Internal Medicine 21: 848-862.

12 Ryu, M. H. and Cha, Y. S. (2003). The effects of a high-fat or high-sucrose diet on serum lipid 13 profiles, hepatic acyl-CoA synthetase, carnitine palmitoyltransferase-I, and the acetyl-CoA 14 carboxylase mRNA levels in rats. Journal of Biochemistry and Molecular Biology 36: 312-318.

15 Sachan, D. S. and Hongu, N. (2000). Increases in VO2max and metabolic markers of fat oxidation 16 by caffeine, carnitine, and choline supplementation in rats. Journal of Nutritional Biochemistry $17 \quad$ 11: $521-526$.

18 Saheb, J. L. and Demers, J. M. (1972). Effect of Lipotropic Factors on Cholesterol Metabolism in 19 Duckling. Annales De Biologie Animale Biochimie Biophysique 12: 149. 149-157

20 Sahyoun, N. R., Jacques, P. F., Zhang, X. L., Juan, W. and McKeown, N. M. (2006). Whole-grain 21 intake is inversely associated with the metabolic syndrome and mortality in older adults. 22 American Journal of Clinical Nutrition 83: 124-131.

23 Sakakibara, K., Shibata, Y., Higashi, T., Sanada, S. and Shoji, J. (1975). Effect of ginseng saponins 24 on cholesterol-metabolism .1. Level and synthesis of serum and liver cholesterol in rats treated 25 with ginsenosides. Chemical and Pharmaceutical Bulletin 23: 1009-1016. 
1 Sakamoto, A., Nishimura, Y., Ono, H. and Sakura, N. (2002). Betaine and homocysteine 2 concentrations in foods. Pediatrics International 44: 409-413.

3 Sakamoto, K., Vucenik, I. and Shamsuddin, A. M. (1993). $\left[{ }^{3} \mathrm{H}\right]$ Phytic acid (inositol hexaphosphate)

4 is absorbed and distributed to various tissues in rats. Journal of Nutrition 123: 713-720.

5 Salama, R. H. M., Nassar, A. Y. A., Nafady, A. A. M. and Mohamed, H. H. T. (2007). A novel 6 therapeutic drug (copper nicotinic acid complex) for non-alcoholic fatty liver. Liver International $7 \quad 27: 454-464$.

8 Samman, S., Sivarajah, G., Man, J., Ahmad, Z., Petocz, P. and Caterson, I. (2002). Supplementation 9 with a mixed fruit and vegetable concentrate increases plasma antioxidant vitamins and lowers 10 plasma homocysteine in men. FASEB Journal 16: A238-A238.

11 Sanchez-Lozada, L., Mu, W., Roncal, C., Sautin, Y., Abdelmalek, M., Reungjui, S., Le, M., 12 Nakagawa, T., Lan, H., Yu, X. and Johnson, R. (2010). Comparison of free fructose and glucose to sucrose in the ability to cause fatty liver. European Journal of Nutrition 49: 1-9.

14 Sandberg, A. S. and Andersson, H. (1988). Effect of dietary phytase on the digestion of phytate in 15 the stomach and small intestine of humans. Journal of Nutrition 118: 469-473.

16 Sandberg, A.-S., Andersson, H., Carlsson, N.-G. and Sandstrom, B. (1987). Degradation products 17 of bran phytate formed during digestion in the human small intestine: effect of extrusion cooking on digestibility. Journal of Nutrition 117: 2061-2065.

19 Sandberg, A. S. and Andlid, T. (2002). Phytogenic and microbial phytases in human nutrition. 20 International Journal of Food Science and Technology 37: 823-833.

21 Sanders, L. M. and Zeisel, S. H. (2007). Choline. Nutrition Today 42: 181-186.

22 Sanz, M. L., Villamiel, M. and Martinez-Castro, I. (2004). Inositols and carbohydrates in different 23 fresh fruit juices. Food Chemistry 87: 325-328.

24 Schade, H. and Saltman, P. (1959). Influence of nicotinic acid on hepatic cholesterol synthesis in rabbits. Proceedings of the Society for Experimental Biology and Medicine 102: 265-267. 
1 Schaefer, A. E., McKibbin, J. M. and Elvehjem, C. A. (1942). Pantothenic acid deficiency studies in 2 dogs. Journal of Biological Chemistry 143: 321-330.

3 Scharrer, E. and Langhans, W. (1986). Control of food intake by fatty acid oxidation. American 4 Journal of Physiology - Regulatory, Integrative and Comparative Physiology 250: R1003-1006.

5 Schatzkin, A., Park, Y., Leitzmann, M. F., Hollenbeck, A. R. and Cross, A. J. (2008). Prospective 6 study of dietary fiber, whole grain foods, and small intestinal cancer. Gastroenterology 135: $7 \quad 1163-1167$.

8 Schneeman, B. O. and Richter, D. (1993). Changes in plasma and hepatic lipids, small-intestinal 9 histology and pancreatic-enzyme activity due to aging and dietary fiber in rats. Journal of $10 \quad$ Nutrition 123: 1328-1337.

11 Schön, H. (1958). Effect of nicotinic acid on the cholesterol contents of rat-livers. Nature 182: 534.

12 Schwarz, J. M., Chen, T. W. and Linfoot, P. (1999). Effect of hydroxycitrate on hepatic de novo 13 lipogenesis, gluconeogenesis and glucose production in obese hyperinsulinemic subjects. 14 Circulation 100 (Suppl. I): 196-197 (abstract n¹015).

15 Schweikart, J., Reimann, J. and Schön, C. (2009). Investigation of niacin on parameters of 16 metabolism in a physiologic dose: randomized, double-blind clinical trial with three different 17 dosages. International Journal of Food Sciences and Nutrition 60: 192 - 202.

18 Schweizer, T. F., Horman, I. and Würsch, P. (1978). Low molecular weight carbohydrates from 19 leguminous seeds; a new disaccharide: Galactopinitol. Journal of the Science of Food and 20 Agriculture 29: 148-154.

21 Schwenk, T. L. and Fisher, M. (1994). Hepatitis caused by low-dose sustained-release niacin. 22 Journal of the American Board of Family Practice 7: 242-244.

23 Scriban, R. (1970). Vitamins of barley, malt and beer. Annales de la Nutrition et de l'Alimentation 24 24: B377-B399. 
1 Sealls, W., Gonzalez, M., Brosnan, M. J., Black, P. N. and DiRusso, C. C. (2008). Dietary 2 polyunsaturated fatty acids (C18:2 $\omega 6$ and $\mathrm{C} 18: 3 \omega 3)$ do not suppress hepatic lipogenesis. 3 Biochimica et Biophysica Acta-Molecular and Cell Biology of Lipids 1781: 406-414.

4 Seetharamaiah, G. S. and Chandrasekhara, N. (1988). Hypocholesterolemic activity of oryzanol in rats. Nutrition Reports International 38: 927-935.

6 Seetharamaiah, G. S. and Chandrasekhara, N. (1993). Comparative hypocholesterolemic activities 7 of oryzanol, curcumin and ferulic acid in rats. Journal of Food Science and Technology-Mysore 30: $249-252$.

9 Seifert, R. M. (1972). Analysis of myoinositol in dry beans by gas-chromatography of its 10 hexaacetate. Journal of the Association of Official Analytical Chemists 55: 1194-1198.

11 Sekiya, M., Yahagi, N., Matsuzaka, T., Najima, Y., Nakakuki, M., Nagai, R., Ishibashi, S., Osuga, 12 J.-i., Yamada, N. and Shimano, H. (2003). Polyunsaturated fatty acids ameliorate hepatic steatosis in obese mice by SREBP-1 suppression. Hepatology 38: 1529-1539.

Seline, K. G. and Johein, H. (2007). The determination of L-carnitine in several food samples. Food Chemistry 105: 793-804.

Sener, G., Balkan, J., Cevikbas, U., Keyer-Uysal, M. and Uysal, M. (2004). Melatonin reduces cholesterol accumulation and prooxidant state induced by high cholesterol diet in the plasma, the liver and probably in the aorta of C57BL/6J mice. Journal of Pineal Research 36: 212-216.

19 Seppala-Lindroos, A., Vehkavaara, S., Hakkinen, A.-M., Goto, T., Westerbacka, J., Sovijarvi, A., Halavaara, J. and Yki-Jarvinen, H. (2002). Fat accumulation in the liver is associated with defects in insulin suppression of glucose production and serum free fatty acids independent of obesity in normal men. Journal of Clinical Endocrinology and Metabolism 87: 3023-3028.

Shamsuddin, A. M. (2002). Anti-cancer function of phytic acid. International Journal of Food Science and Technology 37: 769-782.

Shara, M., Ohia, S., Schmidt, R., Yasmin, T., Zardetto-Smith, A., Kincaid, A., Bagchi, M., 26 Chatterjee, A., Bagchi, D. and Stohs, S. (2004). Physico-chemical properties of a novel (-)- 
1 hydroxycitric acid extract and its effect on body weight, selected organ weights, hepatic lipid 2 peroxidation and DNA fragmentation, hematology and clinical chemistry, and histopathological 3 changes over a period of 90 days. Molecular and Cellular Biochemistry 260: 171-186.

4 Shara, M., Ohia, S. E., Yasmin, T., Zardetto-Smith, A., Kincaid, A., Bagchi, M., Chatterjee, A., 5 Bagchi, D. and Stohs, S. J. (2003). Dose- and time-dependent effects of a novel (-)-hydroxycitric 6 acid extract on body weight, hepatic and testicular lipid peroxidation, DNA fragmentation and 7 histopathological data over a period of 90 days. Molecular and Cellular Biochemistry 254: 3398346.

9 Sharabi, Y. and Eldad, A. (2000). Nonalcoholic fatty liver disease is associated with hyperlipidemia 10 and obesity. American Journal of Medicine 109: 171-171.

11 Shechter, M., Merz, C. N. B., Paul-Labrador, M., Meisel, S. R., Rude, R. K., Molloy, M. D., 12 Dwyer, J. H., Shah, P. K. and Kaul, S. (1999). Oral magnesium supplementation inhibits platelet13 dependent thrombosis in patients with coronary artery disease. American Journal of Cardiology 14 84: 152-156.

15 Shepherd, J., Packard, C. J., Patsch, J. R. and Gotto, A. M. (1979). Effects of nicotinic-acid therapy 16 on plasma high-density lipoprotein subfraction distribution and composition and on 17 apolipoprotein a metabolism. Journal of Clinical Investigation 63: 858-867.

18 Shi, Z., Hu, X., Yuan, B., Hu, G., Pan, X., Dai, Y., Byles, J. E. and Holmboe-Ottesen, G. (2008). 19 Vegetable-rich food pattern is related to obesity in China. International Journal of Obesity 32: $20 \quad 975-984$.

21 Shieh, J., Wu, H., Cheng, K. and Cheng, J. (2009). Melatonin ameliorates high fat diet-induced 22 diabetes and stimulates glycogen synthesis via a $\mathrm{PKC} \zeta$-Akt-GSK3 $\beta$ pathway in hepatic cells. 23 Journal of Pineal Research 47: 339-344.

24 Shils, M. E. and Stewart, W. B. (1954). Development of portal fatty liver in rats on corn diets 25 response to lipotropic agents. Proceedings of the Society for Experimental Biology and Medicine $26 \quad$ 85: $298-303$. 
1 Shimada, M., Hashimoto, E., Taniai, M., Hasegawa, K., Okuda, H., Hayashi, N., Takasaki, K. and 2 Ludwig, J. (2002). Hepatocellular carcinoma in patients with non-alcoholic steatohepatitis. 3 Journal of Hepatology 37: 154-160.

4 Shimizu, H., Oh-I, S., Okada, S. and Mori, M. (2007). Leptin Resistance and Obesity. Endocrine 5 Journal 54: 17-26.

6 Shimotoyodome, A., Haramizu, S., Inaba, M., Murase, T. and Tokimitsu, I. (2005). Exercise and 7 green tea extract stimulate fat oxidation and prevent obesity in mice. Medicine and Science in $8 \quad$ Sports and Exercise 37: 1884-1892.

9 Silverman, J. F., Obrien, K. F., Long, S., Leggett, N., Khazanie, P. G., Pories, W. J., Norris, H. T. 10 and Caro, J. F. (1990). Liver pathology in morbidly obese patients with and without diabetes. 11 American Journal of Gastroenterology 85: 1349-1355.

12 Silverman, J. F., Pories, W. J. and Caro, J. F. (1989). Liver pathology in diabetes-mellitus and 13 morbid-obesity - clinical, pathological, and biochemical considerations. Pathology Annual 24: $14 \quad 275-302$.

15 Singal, S. A. and Eckstein, H. C. (1939). Supplementary proteins and amino acids and dietary 16 production of fatty livers in mice. Proceedings of the Society for Experimental Biology and $17 \quad$ Medicine 41: 512-513.

18 Singal, S. A., Hazan, S. J., Sydenstricker, V. P. and Littlejohn, J. M. (1953). The production of fatty 19 livers in rats on threonine- and lysine-deficient diets. Journal of Biological Chemistry 200: 86720874.

21 Singh, U., Yokota, K., Gupta, C. and Shinozuka, H. (1990). Choline deficiency activates 22 phospholipases A2 and C in rat liver without affecting the activity of protein kinase C. Journal of $23 \quad$ Nutritional Biochemistry 1: 434-439.

24 Siripurkpong, P. and Na-Bangehang, K. (2009). Effects of niacin and chromium on the expression 25 of ATP-binding cassette transporter A1 and apolipoprotein A-1 genes in HepG2 cells. Journal of $26 \quad$ Nutritional Biochemistry 20: 261-268. 
1 Slavin, J. (2003). Why whole grains are protective: biological mechanisms. Proceedings of the $2 \quad$ Nutrition Society 62: 129-134.

3 Slow, S., Donaggio, M., Cressey, P. J., Lever, M., George, P. M. and Chambers, S. T. (2005). The 4 betaine content of New Zealand foods and estimated intake in the New Zealand diet. Journal of $5 \quad$ Food Composition and Analysis 18: 473-485.

6 Smith, C. M. and Song, W. O. (1996). Comparative nutrition of pantothenic acid. Journal of $7 \quad$ Nutritional Biochemistry 7: 312-321.

8 Song, B. J., Moon, K. H., Olsson, N. U. and Salem, N. (2008). Prevention of alcoholic fatty liver 9 and mitochondrial dysfunction in the rat by long-chain poly unsaturated fatty acids. Journal of 10 Hepatology 49: 262-273.

11 Song, B.-L. and DeBose-Boyd, R. A. (2006). Insig-dependent ubiquitination and degradation of 312 hydroxy-3-methylglutaryl coenzyme A reductase stimulated by $\alpha$ - and $\gamma$-tocotrienols. Journal of 13 Biological Chemistry 281: 25054-25061.

14 Sorrell, M. F., Baker, H., Tuma, D. J., Frank, O. and Barak, A. J. (1976). Potentiation of ethanol 15 fatty liver in rats by chronic administration of nicotinic-acid. Biochimica et Biophysica Acta $\mathbf{4 5 0}$ : $16 \quad 231-238$.

17 Sosulski, F. W., Elkowicz, L. and Reichert, R. D. (1982). Oligosaccharides in eleven legumes and 18 their air-classified protein and starch fractions. Journal of Food Science 47: 498-502.

19 Souci, S. W., Fachmann, W. and Kraut, H. (2008). Food composition and nutritional tables, $7^{\text {th }}$ 20 revised and completed edition. Taylor and Francis, Medpharm Scientific Publishers, Stuttgart, 21 Germany.

22 Spadaro, L., Magliocco, O., Spampinato, D., Piro, S., Oliveri, C., Alagona, C., Papa, G., Rabuazzo, 23 A. M. and Purrello, F. (2008). Effects of n-3 polyunsaturated fatty acids in subjects with 24 nonalcoholic fatty liver disease. Digestive and Liver Disease 40: 194-199. 
1 Spaniol, M., Kaufmann, P., Beier, K., Wuthrich, J., Torok, M., Scharnagl, H., Marz, W. and 2 Krahenbuhl, S. (2003). Mechanisms of liver steatosis in rats with systemic carnitine deficiency 3 due to treatment with trimethylhydraziniumpropionate. Journal of Lipid Research 44: 144-153.

4 Sreekumar, R., Wiesner, R. H., Nagorney, D. M., Rosen, C. B. and Charlton, M. R. (2001). Unique 5 hepatic gene expression in NASH - Candidates for a genetic basis of disease. Gastroenterology $6 \quad$ 120: 580 .

7 St. Greif, von and Wenning, F. (1954). Lipotropic effects of vitamin B12 and folic acid. Wiener Medizinische Wochenschrift 104: 35-36.

9 Stanner, S., Hughes, J., Kelly, C. and Buttriss, J. (2004). A review of the epidemiological evidence 10 for the 'antioxidant hypothesis'. Public Health Nutrition 7: 407-422.

11 Steadman, K. J., Burgoon, M. S., Schuster, R. L., Lewis, B. A., Edwardson, S. E. and Obendorf, R. 12 L. (2000). Fagopyritols, D-chiro-inositol, and other soluble carbohydrates in buckwheat seed 13 milling fractions. Journal of Agricultural and Food Chemistry 48: 2843-2847.

14 Steffen, L. M., Jacobs, D. R., Jr., Stevens, J., Shahar, E., Carithers, T. and Folsom, A. R. (2003). 15 Associations of whole-grain, refined-grain, and fruit and vegetable consumption with risks of all16 cause mortality and incident coronary artery disease and ischemic stroke: the Atherosclerosis Risk 17 in Communities (ARIC) Study. American Journal of Clinical Nutrition 78: 383-390.

18 Stella, C., Beckwith-Hall, B., Cloarec, O., Holmes, E., Lindon, J. C., Powell, J., van der Ouderaa, 19 F., Bingham, S., Cross, A. J. and Nicholson, J. K. (2006). Susceptibility of human metabolic 20 phenotypes to dietary modulation. Journal of Proteome Research 5: 2780-2788.

21 Stern, R. H. (2007). The role of nicotinic acid metabolites in flushing and hepatotoxicity. Journal of 22 Clinical Lipidology 1: 191-193.

23 Stewart, J. R., Fryer, E. B. and Fryer, H. C. (1987). Effects of dietary fiber, carbohydrate, lipid and 24 protein-levels on serum and liver lipids in rats. Journal of Nutrition 117: 650-659.

25 Story, J. A., Baldino, A., Czarnecki, S. K. and Kritchevsky, D. (1981). Modification of liver 26 cholesterol accumulation by dietary fiber in rats. Nutrition Reports International 24: 1213-1219. 
1 Subramanian, P., Mirunalini, S., Pandi-Perumal, S. R., Trakht, I. and Cardinali, D. P. (2007).

2 Melatonin treatment improves the antioxidant status and decreases lipid content in brain and liver 3 of rats. European Journal of Pharmacology 571: 116-119.

4 Subramanian, V., Byrne, J. J., Kaye, P., Daykin, C. A. and Aithal, G. P. (2008). Serum 5 metabolomics reveals novel metabolic markers of non alcoholic fatty liver disease. Hepatology $6 \quad$ 48: $811 \mathrm{~A}\left(\right.$ Abstract $\left.\mathrm{N}^{\circ} 1130\right)$.

7 Sugano, M., Tanaka, K. and Ide, T. (1982). Secretion of cholesterol, triglyceride and apolipoprotein 8 A-I by isolated perfused liver from rats fed soybean protein and casein or their amino acid 9 mixtures. Journal of Nutrition 112: 855-862.

10 Sugatani, J., Wada, T., Osabe, M., Yamakawa, K., Yoshinari, K. and Miwa, M. (2006). Dietary 11 inulin alleviates hepatic steatosis and xenobiotics-induced liver injury in rats fed a high-fat and 12 high-sucrose diet: association with the suppression of hepatic cytochrome P450 and hepatocyte 13 nuclear factor 4 alpha expression. Drug Metabolism and Disposition 34: 1677-1687.

14 Sugiyama, K., Kanamori, H., Akachi, T. and Yamakawa, A. (1996). Amino acid composition of 15 dietary proteins affects plasma cholesterol concentration through alteration of hepatic 16 phospholipid metabolism in rats fed a cholesterol-free diet. Journal of Nutritional Biochemistry 7 : $17 \quad 40-48$

18 Suh, M. H., Yoo, S. H., Chang, P. S. and Lee, H. G. (2005). Antioxidative activity of 19 microencapsulated $\gamma$-oryzanol on high cholesterol-fed rats. Journal of Agricultural and Food 20 Chemistry 53: 9747-9750.

21 Sullivan, A., Triscari, J., Hamilton, J. and Miller, O. (1974a). Effect of (-)-hydroxycitrate upon the 22 accumulation of lipid in the rat: II. Appetite. Lipids 9: 129-134.

23 Sullivan, A., Triscari, J., Hamilton, J., Miller, O. and Wheatley, V. (1974b). Effect of (-)24 hydroxycitrate upon the accumulation of lipid in the rat: I. Lipogenesis. Lipids 9: 121-128. 
1 Sullivan, A., Triscari, J. and Spiegel, J. (1977). Metabolic regulation as a control for lipid disorders.

2 II. Influence of (-)-hydroxycitrate on genetically and experimentally induced hypertriglyceridemia 3 in the rat. American Journal of Clinical Nutrition 30: 777-784.

4 Sullivan, A. C., Hamilton, J. G., Miller, O. N. and Wheatley, V. R. (1972). Inhibition of lipogenesis

5 in rat liver by (-)-hydroxycitrate. Archives of Biochemistry and Biophysics 150: 183-190.

6 Sullivan, C. and Triscari, J. (1977). Metabolic regulation as a control for lipid disorders. I. Influence 7 of (--)-hydroxycitrate on experimentally induced obesity in the rodent. American Journal of $8 \quad$ Clinical Nutrition 30: 767-776.

9 Summers, P. S. and Weretilnyk, E. A. (1993). Choline synthesis in spinach in relation to salt stress. 10 Plant Physiology 103: 1269-1276.

11 Sundkvist, G., Dahlin, L. B., Nilsson, H., Eriksson, K. F., Lindgarde, F., Rosen, I., Lattimer, S. A., 12 Sima, A. A. F., Sullivan, K. and Greene, D. A. (2000). Sorbitol and myo-inositol levels and morphology of sural nerve in relation to peripheral nerve function and clinical neuropathy in men with diabetic, impaired, and normal glucose tolerance. Diabetic Medicine 17: 259-268.

Suzuki, K., Nakamura, T., Fujita, M., Iwami, T., Abe, M. and Okada, M. (1976). Factors affecting liver lipid-content in pyridoxine-deficient rats. 1. Dietary-protein levels. Journal of Nutritional Science and Vitaminology 22: 291-298.

Takeuchi, H., Nakamoto, T., Mori, Y., Kawakami, M., Mabuchi, H., Ohishi, Y., Ichikawa, N., Koike, A. and Masuda, K. (2001). Comparative effects of dietary fat types on hepatic enzyme activities related to the synthesis and oxidation of fatty acid and to lipogenesis in rats. Bioscience, Biotechnology, and Biochemistry 65: 1748-1754.

Tandy, S., Chung, R. W. S., Wat, E., Kamili, A., Berge, K., Griinari, M. and Cohn, J. S. (2009). Dietary krill oil supplementation reduces hepatic steatosis, glycemia, and hypercholesterolemia in high-fat-fed mice. Journal of Agricultural and Food Chemistry 57: 9339-9345. 
1 Tato, F., Vega, G. L. and Grundy, S. M. (1998). Effects of crystalline nicotinic acid-induced hepatic 2 dysfunction on serum low-density lipoprotein cholesterol and lecithin cholesteryl acyl transferase. 3 American Journal of Cardiology 81: 805-807.

4 Taysi, S., Koc, M., Buyukokuroglu, M. E., Altinkaynak, K. and Sahin, Y. N. (2003). Melatonin 5 reduces lipid peroxidation and nitric oxide during irradiation-induced oxidative injury in the rat liver. Journal of Pineal Research 34: 173-177.

7 The Alpha-Tocopherol, Beta Carotene Cancer Prevention Study Group (1994). The effect of vitamin $\mathrm{E}$ and beta carotene on the incidence of lung cancer and other cancers in male smokers. New England Journal of Medicine 330: 1029-1035.

10 Thomas, M., Leelamma, S. and Kurup, P. A. (1983). Effect of blackgram fiber (phaseolus-mungo) 11 on hepatic hydroxymethylglutaryl-CoA reductase-activity, cholesterogenesis and cholesterol 12 degradation in rats. Journal of Nutrition 113: 1104-1108.

13 Thompson, H. J., Heimendinger, J., Diker, A., O'Neill, C., Haegele, A., Meinecke, B., Wolfe, P., 14 Sedlacek, S., Zhu, Z. J. and Jiang, W. Q. (2006). Dietary botanical diversity affects the reduction 15 of oxidative biomarkers in women due to high vegetable and fruit intake. Journal of Nutrition 16 136: $2207-2212$.

17 Thompson, H. J., Heimendinger, J., Gillette, C., Sedlacek, S. M., Haegele, A., O'Neill, C. and 18 Wolfe, P. (2005). In vivo investigation of changes in biomarkers of oxidative stress induced by 19 plant food rich diets. Journal of Agricultural and Food Chemistry 53: 6126-6132.

20 Thor, H., Smith, M. T., Hartzell, P., Bellomo, G., Jewell, S. A. and Orrenius, S. (1982). The 21 metabolism of menadione (2-methyl-1,4-naphtoquinone) by isolated hepatocytes. A study of the 22 implication of oxidative stress in intact cells. Journal of Biological Chemistry 257: 12419-12425.

23 Thuillier, J. (1956). Betaine, a lipotropic factor. Le Concours Médical 78: 243-244.

24 Thurston, J. H. and Hauhart, R. E. (1992). Amelioration of adverse-effects of valproic acid on ketogenesis and liver coenzyme-a metabolism by cotreatment with pantothenate and carnitine in developing mice - possible clinical-significance. Pediatric Research 31: 419-423. 
1 Tokmakjian, S. and Haines, D. S. M. (1979). Influence of dietary choline intake upon liver 2 ethanolamine metabolism. Canadian Journal of Biochemistry 57: 566-572.

3 Tomita, K., Okuhara, Y., Shigematsu, N., Suh, H. and Lim, K. (2003). (-)-Hydroxycitrate ingestion 4 increases fat oxidation during moderate intensity exercise in untrained men. Bioscience, $5 \quad$ Biotechnology and Biochemistry 67: 1999-2001.

6 Toussant, M. J., Wilson, M. D. and Clarke, S. D. (1981). Coordinate suppression of liver acetyl7 CoA carboxylase and fatty acid synthetase by polyunsaturated fat. Journal of Nutrition 111: 146$8 \quad 153$

9 Tucker, H. F. and Eckstein, H. C. (1937). The effect of supplementary methionine and cystine on 10 the production of fatty livers by diet. Journal of Biological Chemistry 121: 479-484.

11 Tucker, H. F. and Eckstein, H. C. (1938). The effect of supplementary lysine, methionine, and 12 cystine on the production of fatty livers by high fat diets containing gliadin. Journal of Biological 13 Chemistry 126: 117-123.

14 Turchetto, E., Infante, R. and Sechi, A. M. (1955). Effect of thyroxin on lipotropic activity of 15 pantothenic acid. Bollettino- Societa Italiana Biologia Sperimentale (Napoli) 31: 233-234.

16 Tyner, E. P., Lewis, H. B. and Eckstein, H. C. (1950). Niacin and the ability of cystine to augment 17 deposition of liver fat. Journal of Biological Chemistry 187: 651-654.

18 USDA (2004). USDA database for the proanthocyanidin content of selected foods. United States 19 Department of Agriculture (USDA). $<$ http://www.nal.usda.gov/fnic/foodcomp/Data/PA/PA.pdf $>$. 20 USDA (2005a). USDA national nutrient database for standard reference, release 18. United States 2

$$
\text { Department of Agriculture }
$$

(USDA).

$22<$ http://www.nal.usda.gov/fnic/foodcomp/Data/SR18/reports/sr18page.htm>

23 USDA (2005b). USDA national nutrient database for standard reference, release 18: cereal grains 24 and pasta. United States Department of Agriculture (USDA). < 25 http://www.nal.usda.gov/fnic/foodcomp/Data/SR18/reports/sr18fg20.pdf $>$. 
1 USDA (2005c). USDA national nutrient database for standard reference, release 18: legumes and 2 legume products. United States Department of Agriculture (USDA). < 3 http://www.nal.usda.gov/fnic/foodcomp/Data/SR18/reports/sr18fg16.pdf $>$.

4 USDA (2005d). USDA national nutrient database for standard reference, release 18: nuts and seed 5 products. United States Department of Agriculture (USDA). < 6 http://www.nal.usda.gov/fnic/foodcomp/Data/SR18/reports/sr18fg12.pdf $>$.

7 USDA (2005e). USDA national nutrient database for standard reference, release 18: fats and oils. 8 United States Department of Agriculture (USDA). < 9 http://www.nal.usda.gov/fnic/foodcomp/Data/SR18/reports/sr18fg04.pdf $>$.

10 USDA (2007). USDA database for the flavonoid content of selected foods, release 2.1. United 11 States Department of Agriculture $\quad$ (USDA). $12<$ http://www.nal.usda.gov/fnic/foodcomp/Data/Flav/Flav02-1.pdf $>$.

13 USDA(2008). USDA database for the isoflavone content of selected foods, release 2.0. United
States
Department
of
Agriculture
$(\mathrm{USDA}) .<$

15 http://www.ars.usda.gov/SP2UserFiles/Place/12354500/Data/isoflav/Isoflav_R2.pdf>.

16 USDA (2008). USDA database for the choline content of common foods, release 2. United States

17 Department of Agriculture $\quad$ (USDA).

$18<$ http://www.nal.usda.gov/fnic/foodcomp/Data/Choline/Choln02.pdf $>$.

19 Ueshima, T., Shigata, Y., Wada, M., Oji, K. and Yoshida, T. (1956). Studies on the metabolism of 20 pantothenic acid in liver damage. I. Urinary excretion of pantothenic acid in patients with various 21 liver diseases and its correlation with liver function. Journal of Vitaminology 2: 299-306.

22 Ueshima, T., Unno, H., Shigeta, Y., Wada, M., Oji, K. and Yoshida, T. (1958). Studies on the 23 metabolism of pantothenic acid in liver damage. II. Pantothenic acid treatment in patients with 24 liver disease. Journal of Vitaminology 4: 149-155. 
1 Vaishwanar, I., Jiddewar, G. G., Shukla, R. D. and Kowale, C. N. (1972). Effect of nicotinic-acid 2 on serum and hepatic lipids in experimentally induced fatty liver. Indian Journal of Experimental 3 Biology 10: 428-430.

4 Valtuena, S., Pellegrini, N., Ardigo, D., Del Rio, D., Numeroso, F., Scazzina, F., Monti, L., 5 Zavaroni, I. and Brighenti, F. (2006). Dietary glycemic index and liver steatosis. American $6 \quad$ Journal of Clinical Nutrition 84: 136-142.

7 van de Vijver, L. P. L., van den Bosch, L. M. C., van den Brandt, P. A. and Goldbohm, R. A. 8 (2009). Whole-grain consumption, dietary fibre intake and body mass index in the Netherlands 9 cohort study. European Journal of Clinical Nutrition 63: 31-38.

10 Van den Veyver, I. B. (2002). Genetic effects of methylation diets. Annual Review of Nutrition 22 : $11255-282$.

12 van der Hoorn, J. W. A., de Haan, W., Berbee, J. F. P., Havekes, L. M., Jukema, J. W., Rensen, P. 13 C. N. and Princen, H. M. G. (2008). Niacin increases hdl by reducing hepatic expression and 14 plasma levels of cholesteryl ester transfer protein in APOE*3Leiden.CETP mice. Arteriosclerosis 15 Thrombosis and Vascular Biology 28: 2016-2022.

16 van Duijnhoven, F. J., Bueno-De-Mesquita, H. B., Ferrari, P., Jenab, M., Boshuizen, H. C., Ros, M. 17 M., Casagrande, C., Tjonneland, A., Olsen, A., Overvad, K., Thorlacius-Ussing, O., Clavel18 Chapelon, F., Boutron-Ruault, M.-C., Morois, S., Kaaks, R., Linseisen, J., Boeing, H., Nothlings, 19 U., Trichopoulou, A., Trichopoulos, D., Misirli, G., Palli, D., Sieri, S., Panico, S., Tumino, R., 20 Vineis, P., Peeters, P. H., van Gils, C. H., Ocke, M. C., Lund, E., Engeset, D., Skeie, G., Suarez, 21 L. R., Gonzalez, C. A., Sanchez, M.-J., Dorronsoro, M., Navarro, C., Barricarte, A., Berglund, G., 22 Manjer, J., Hallmans, G., Palmqvist, R., Bingham, S. A., Khaw, K.-T., Key, T. J., Allen, N. E., 23 Boffetta, P., Slimani, N., Rinaldi, S., Gallo, V., Norat, T. and Riboli, E. (2009). Fruit, vegetables, 24 and colorectal cancer risk: the European Prospective Investigation into Cancer and Nutrition. 25 American Journal of Clinical Nutrition 89: 1441-1452. 
1 van Hung, L. (1953). Acetylation of p-aminobenzoic acid in fatty degeneration of the liver in rat. $2 \quad$ Journal of Physiology - Paris 45.

3 van Loon, L. J. C., van Rooijen, J. J. M., Niesen, B., Verhagen, H., Saris, W. H. M. and 4 Wagenmakers, A. J. M. (2000). Effects of acute (-)-hydroxycitrate supplementation on substrate 5 metabolism at rest and during exercise in humans. American Journal of Clinical Nutrition $\mathbf{7 2 :}$ $6 \quad 1445-1450$.

7 Varady, K. A., Wang, Y. and Jones, P. J. H. (2003). Role of policosanols in the prevention and 8 treatment of cardiovascular disease. Nutrition Reviews 61: 376-383.

9 Venables, M. C., Hulston, C. J., Cox, H. R. and Jeukendrup, A. E. (2008). Green tea extract 10 ingestion, fat oxidation, and glucose tolerance in healthy humans. American Journal of Clinical $11 \quad$ Nutrition 87: 778-784.

12 Venn, B. J. and Mann, J. I. (2004). Cereal grains, legumes and diabetes. European Journal of 13 Clinical Nutrition 58: 1443-1461.

14 Villegas, R., Shu, X. O., Gao, Y.-T., Yang, G., Elasy, T., Li, H. and Zheng, W. (2008). Vegetable 15 but not fruit consumption reduces the risk of type 2 diabetes in chinese women. Journal of $16 \quad$ Nutrition 138: 574-580.

17 Vucenik, I. and Shamsuddin, A. M. (1994). [ $\left.{ }^{3} \mathrm{H}\right]$ inositol hexaphosphate (phytic acid) is rapidly 18 absorbed and metabolized by murine and human malignant cells in vitro. Journal of Nutrition 19 124: $861-868$.

20 Vuppalanchi, R. and Chalasani, N. (2009). Nonalcoholic fatty liver disease and nonalcoholic 21 steatohepatitis: Selected practical issues in their evaluation and management. Hepatology 49: 30622317.

23 Walsh, M. C., Brennan, L., Pujos-Guillot, E., Sebedio, J. L., Scalbert, A., Fagan, A., Higgins, D. G. 24 and Gibney, M. J. (2007). Influence of acute phytochemical intake on human urinary 25 metabolomic profiles. American Journal of Clinical Nutrition 86: 1687-1693. 
1 Wang, W., Basinger, A., Neese, R. A., Shane, B., Myong, S. A., Christiansen, M. and Hellerstein,

2 M. K. (2001). Effect of nicotinic acid administration on hepatic very low density lipoprotein3 triglyceride production. American Journal of Physiology-Endocrinology and Metabolism 280: $4 \quad$ E540-E547.

5 Wang, X., Song, K.-S., Guo, Q.-X. and Tian, W.-X. (2003). The galloyl moiety of green tea 6 catechins is the critical structural feature to inhibit fatty-acid synthase. Biochemical $7 \quad$ Pharmacology 66: 2039-2047.

8 Wang, X. and Tian, W. (2001). Green tea epigallocatechin gallate: a natural inhibitor of fatty-acid $9 \quad$ synthase. Biochemical and Biophysical Research Communications 288: 1200-1206.

10 Warembourg, H. and Bertrand, M. (1964). Clinical study of a lipotropic formula: Ornitaine. Lille $11 \quad$ Med 37: 285-289.

12 Watson, J. A., Fang, M. and Lowenstein, J. M. (1969). Tricarballylate and hydroxycitrate: substrate 13 and inhibitor of ATP: Citrate oxaloacetate lyase. Archives of Biochemistry and Biophysics 135: $14 \quad 209-217$.

15 Wegkamp, A., van Oorschot, W., de Vos, W. M. and Smid, E. J. (2007). Characterization of the 16 role of para-aminobenzoic acid biosynthesis in folate production by Lactococcus lactis. Applied 17 and Environmental Microbiology 73: 2673-2681.

18 Welsh, A. L. and Ede, M. (1961). Inositol hexanicotinate for improved nicotinic acid therapy. 19 Preliminary report. International Record of Medicine 174: 9-15.

20 Weltman, M. D., Farrell, G. C. and Liddle, C. (1996). Increased hepatocyte CYP2E1 expression in 21 a rat nutritional model of hepatic steatosis with inflammation. Gastroenterology 111: 1645-1653. 22 Wenru, T., Changle, Z., Li, S. J., Zhou, J. P., Yang, H. J., Wang, T., Hu, Q. D., Bai, Y. M., Ma, C. 23 C. and Yang, D. J. (1994). Efficacy of oral nicotinic acid and choline in the treatment and 24 prevention of fatty liver in dairy cow. Journal of Northeast Agricultural University 1: 42-49.

25 Williams, M. A., Chu, L. C., McIntosh, D. J. and Hincenbe.I (1968). Effects of dietary fat level on 26 pantothenate depletion and liver fatty acid composition in rat. Journal of Nutrition 94: 377-382. 
1 Williams, P. G., Grafenauer, S. J. and O'Shea, J. E. (2008). Cereal grains, legumes, and weight 2 management: a comprehensive review of the scientific evidence. Nutrition Reviews 66: 171-182.

3 Wirtschafter, Z. T. and Walsh, J. R. (1962). Hepatocellular lipoid changes in pantothenic acid 4 deficiency. American Journal of Clinical Nutrition 10: 525-530.

5 Wishart, D. S., Tzur, D., Knox, C., Eisner, R., Guo, A. C., Young, N., Cheng, D., Jewell, K., Arndt, 6 D., Sawhney, S., Fung, C., Nikolai, L., Lewis, M., Coutouly, M. A., Forsythe, I., Tang, P., 7 Shrivastava, S., Jeroncic, K., Stothard, P., Amegbey, G., Block, D., Hau, D. D., Wagner, J., 8 Miniaci, J., Clements, M., Gebremedhin, M., Guo, N., Zhang, Y., Duggan, G. E., MacInnis, G. 9 D., Weljie, A. M., Dowlatabadi, R., Bamforth, F., Clive, D., Greiner, R., Li, L., Marrie, T., Sykes, 10 B. D., Vogel, H. J. and Querengesser, L. (2007). HMDB: the human metabolome database. $11 \quad$ Nucleic Acids Research 35: D521-D526.

12 Wojcicki, J., Samochowiec, L., Kadlubowska, D. and Lutomski, J. (1977). Studies on saponin 13 fraction from root of Aralia Mandshurica Rupr. et Maxim. Part IV. Influence of saponosides on 14 content of lipids in blood serum and liver in experimental hyperlipemia. Herba Polonica 23: 28515289.

16 Woodruff, C. W., Clark, S. L., Jr. and Bridgeforth, E. B. (1953). Folic acid deficiency in the Guinea 17 pig. Journal of Nutrition 51: 23-34.

18 Wright, R. S., Anderson, J. W. and Bridges, S. R. (1990). Propionate inhibits hepatocyte lipid19 synthesis. Proceedings of the Society for Experimental Biology and Medicine 195: 26-29.

20 Wu, D., Keller, W. L. and Park, C. S. (1998). Lipotrope deficiency inhibits cell growth and induces 21 programmed cell death in human breast cancer cell line MCF-7. Nutrition and Cancer-an 22 International Journal 32: 13-19.

23 Wu, H., Dai, Q., Shrubsole, M. J., Ness, R. M., Schlundt, D., Smalley, W. E., Chen, H., Li, M., 24 Shyr, Y. and Zheng, W. (2009). Fruit and vegetable intakes are associated with lower risk of 25 colorectal adenomas. Journal of Nutrition 139: 340-344. 
$1 \mathrm{Wu}, \mathrm{L} . \mathrm{L}$. and $\mathrm{Wu}, \mathrm{J} . \mathrm{T}$. (2002). Hyperhomocysteinemia is a risk factor for cancer and a new 2 potential tumor marker. Clinica Chimica Acta 322: 21-28.

3 Wu, X., Beecher, G. R., Holden, J. M., Haytowitz, D. B., Gebhardt, S. E. and Prior, R. L. (2004a).

4 Lipophilic and hydrophilic antioxidant capacities of common foods in the united states. Journal of $5 \quad$ Agricultural and Food Chemistry 52: 4026-4037.

6 Wu, X. L., Gu, L. W., Holden, J., Haytowitz, D. B., Gebhardt, S. E., Beecher, G. and Prior, R. L. 7 (2004b). Development of a database for total antioxidant capacity in foods: a preliminary study. Journal of Food Composition and Analysis 17: 407-422.

9 Wutzke, K. D. and Lorenz, H. (2004). The effect of L-carnitine on fat oxidation, protein turnover, 10 and body composition in slightly overweight subjects. Metabolism-Clinical and Experimental 53 : $11 \quad 1002-1006$

12 Xin, Y. N., Xuan, S. Y., Zhang, J. H., Zheng, M. H. and Guan, H. S. (2008). Omega-3 13 polyunsaturated fatty acids: a specific liver drug for non-alcoholic fatty liver disease (NAFLD). $14 \quad$ Medical Hypotheses 71: 820-821.

15 Yagi, K. and Kotaki, A. (1969). The effect of massive doses of myo-inositol on hepatic 16 phospholipid metabolism. Annals of the New York Academy of Sciences 165: 710-725.

17 Yang, J., Xu, G. W., Zheng, Y. F., Kong, H. W., Pang, T., Lv, S. and Yang, Q. (2004). Diagnosis of 18 liver cancer using HPLC-based metabonomics avoiding false-positive result from hepatitis and 19 hepatocirrhosis diseases. Journal of Chromatography B-Analytical Technologies in the $20 \quad$ Biomedical and Life Sciences 813: 59-65.

21 Yang, L. and Kadowaki, M. (2009). Effects of rice proteins from two cultivars, koshihikari and 22 shunyo, on hepatic cholesterol secretion by isolated perfused livers of rats fed cholesterol23 enriched diets. Annals of Nutrition and Metabolism 54: 283-290.

24 Yang, L., Kumagai, T., Kawamura, H., Watanabe, T., Kubota, M., Fujimura, S., Watanabe, R. and 25 Kadowaki, M. (2007). Effects of rice proteins from two cultivars, koshihikari and shunyo, on 
1 cholesterol and triglyceride metabolism in growing and adult rats. Bioscience, Biotechnology, and $2 \quad$ Biochemistry 71: 694-703.

3 Yang, M.-Y., Peng, C.-H., Chan, K.-C., Yang, Y.-S., Huang, C.-N. and Wang, C.-J. (2010). The 4 hypolipidemic effect of Hibiscus sabdariffa polyphenols via inhibiting lipogenesis and promoting $5 \quad$ hepatic lipid clearance. Journal of Agricultural and Food Chemistry 58: 850-859.

6 Yao, Z. and Vance, D. (1988). The active synthesis of phosphatidylcholine is required for very low 7 density lipoprotein secretion from rat hepatocytes. Journal of Biological Chemistry 263: 299883004.

9 Yao, Z. and Vance, D. (1989). Head group specificity in the requirement of phosphatidylcholine 10 biosynthesis for very low density lipoprotein secretion from cultured hepatocytes. Journal of 11 Biological Chemistry 264: 11373-11380.

12 Yao, Z. and Vance, D. E. (1990). Reduction in VLDL, but not HDL, in plasma of rats deficient in 13 choline. Biochemistry and Cell Biology-Biochimie et Biologie Cellulaire 68: 552-558.

14 Yasuhara, M., Ohama, T., Matsuki, N., Saito, H., Shiga, J., Inoue, K., Kurokawa, K. and Teramoto, 15 T. (1991). Induction of fatty liver by fasting in suncus. Journal of Lipid Research 32: 887-891.

16 Yatsuji, S., Hashimoto, E., Toban, M., Takakura, M., Taniai, M., Tokushige, K., Shiratori, K., 17 Shibata, N. and Kobayashi, M. (2006). Liver carcinogenesis in non-alcoholic steatohepatitis 18 (NASH) - Relation to oxidative stress and apoptosis. Gastroenterology 130: A819-A819.

19 Yeh, Y. Y. (1976). Nicotinic-acid reverses fasting ketosis by lowering level of cyclic-AMP. Life 20 Sciences 18: 33-38.

21 Yeh, Y.-Y. and Liu, L. (2001). Cholesterol-lowering effect of garlic extracts and organosulfur 22 compounds: human and animal studies. Journal of Nutrition 131: 989S-993.

23 Yeh, Y.-Y. and Yeh, S.-M. (1994). Garlic reduces plasma lipids by inhibiting hepatic cholesterol 24 and triacylglycerol synthesis. Lipids 29: 189-193.

25 Yokoishi, T. and Tanimoto, S. (1994). Seed-germination of the halophyte Suaeda japonica under 26 salt stress. Journal of Plant Research 107: 385-388. 
1 Yokota, F., Esashi, T., Takahash.S and Suzue, R. (1974). Effects of excess methionine and glycine 2 on incorporation of sodium acetate- $1-{ }^{14} \mathrm{C}$ into lipid of rat liver. Nutrition Reports International 10: $3 \quad 405-408$.

4 York, L. W., Puthalapattu, S. and Wu, G. Y. (2009). Nonalcoholic fatty liver disease and low5 carbohydrate diets. Annual Review of Nutrition 29: 365-379.

6 Young, R. J., Lucas, C. C., Patterson, J. M. and Best, C. H. (1965). Lipotropic dose-response 7 studies in rats: comparisons of choline, betaine, and methionine. Canadian Journal of $8 \quad$ Biochemistry and Physiology 34: 713.

9 Youssef, J., Cunningham, M. L. and Badr, M. (1994). Down-regulation of hepatic peroxisomal 10 beta-oxidation due to pantothenic acid-deficiency. FASEB Journal 8: A736-A736.

11 Zeisel, S., Da Costa, K., Franklin, P., Alexander, E., Lamont, J., Sheard, N. and Beiser, A. (1991). 12 Choline, an essential nutrient for humans. FASEB Journal 5: 2093-2098.

13 Zeisel, S. H. (1981). Dietary choline: biochemistry, physiology, and pharmacology. Annual Review 14 of Nutrition 1: 95-121.

15 Zeisel, S. H. and Costa, K.-A. d. (2009). Choline: an essential nutrient for public health. Nutrition 16 Reviews 67: 615-623.

17 Zeisel, S. H., Mar, M. H., Howe, J. C. and Holden, J. M. (2003). Concentrations of choline18 containing compounds and betaine in common foods. Journal of Nutrition 133: 1302-1307.

19 Zhang, L.-H., Kamanna, V. S., Zhang, M. C. and Kashyap, M. L. (2008). Niacin inhibits surface 20 expression of ATP synthase $\beta$ chain in HepG2 cells: implications for raising HDL. Journal of $21 \quad$ Lipid Research 49: 1195-1201.

22 Zilversmit, D. B. and Diluzio, N. R. (1958). The role of choline in the turnover of phospholipids. 23 American Journal of Clinical Nutrition 6: 235-241.

24 Zivkovic, A. M., German, J. B., Esfandiari, F. and Halsted, C. H. (2009). Quantitative lipid 25 metabolomic changes in alcoholic micropigs with fatty liver disease. Alcoholism-Clinical and 26 Experimental Research 33: 751-758. 


\section{Figure captions}

2 Figure 1 Molecular structure of main lipotropes and of phytochemicals for which at least one

3 significant hepatic total lipids/fat or triglyceride content reduction has been reported in animal 4 studies. Gamma $(\gamma)$-oryzanol is a mixture of ferulic acid esters of triterpene alcohols and sterols. 5 ABBREVIATIONS: SCFA, Short-Chain Fatty Acid; PUFA: Poly-Unsaturated Fatty Acid.

17 precursors lysine and methionine, $2^{\circ}$ ) inhibiting activity of enzymes involved in FA and TG 18 syntheses (i.e. ACC and DGAT), $3^{\circ}$ ) up-regulating expression of genes that code for PPAR $\alpha$; and $194^{\circ}$ ) reducing the release of FFA in plasma through inhibition of catecholamine stimulation of TG 20 lipolysis in adipose tissue; mechanisms by which niacin may inhibit cholesterol synthesis and 21 favour and reduce efflux of respectively Apo A (HDL)- and Apo B (LDL and VLDL)-containing 22 lipoproteins outside livers are also presented. D - The lipotropic effects of other phytochemicals 23 which is mainly based on the up- and down-regulation of gene expression for enzymes and/or 24 transcription factors involved respectively in FA oxidation and synthesis, but which is also based 25 on the specific actions of fiber on incorporation of acetate into cholesterol and FA, of HCA on CCE activity inhibition, of melatonin on decreased oxidative stress and insulino-resistance and of 
1 oligofructose on FA re-esterification inhibition. Figures 1 A-D have been mainly elaborated from

2 results presented in Supplemental Tables 1-4. ABBREVIATIONS: ABCA, ATP-Binding Cassette

3 Transporter; ACC, Acetyl-CoA Carboxylase; ACO, Acyl-CoA Oxidase; AMP, Adenosine

4 MonoPhosphate; Apo A, Apolipoprotein A; Apo B, Apolipoprotein B; ATP, Adenosine

5 TriPhosphate; ATPCL/CCE, ATP-Citrate Lyase/Citrate Cleavage Enzyme; BHMT, Betaine

6 Homocysteine MethylTransferase; CE, Cholesteryl Ester; CETP, Cholesteryl Ester Transfer

7 Protein; CoA, Coenzyme A; CPT, Carnitine Palmitoyl Transferase; DGAT, DiacylGlycerol O8 AcylTransferase; FA, Fatty Acid; FAS, Fatty Acid Synthase; FC, Free Cholesterol; FFA, Free

9 Fatty Acid; Glycerol 3-P, Glycerol 3-Phosphate; G6PDH, Glucose-6-Phosphate-DesHydrogenase;

10 GSH, reduced glutathione; HCA, HydroxyCitric Acid; HDL, High Density Lipoprotein; LDL, Low 11 Density Lipoprotein; ME, Malic Enzyme; Mg, Magnesium; MS, Methionine Synthetase; MUFA, 12 Mono-Unsaturated Fatty Acid; NF- $\kappa$ B, Nuclear Factor Kappa B; PEMT, 13 PhosphatidylEthanolamine- $N$-MethylTransferase; PP, PyroPhosphate; PPAR $\alpha$, Peroxisome 14 Proliferator Activated Receptor alpha; PUFA, PolyUnsaturated fatty Acid; SREBP, Sterol 15 Regulatory Element Binding Protein; TC, Total Cholesterol; TG, triglyceride; THF, 16 TetraHydroFolate; VLDL, Very Low Density Lipoprotein.

18 Figures 3 A-C. Percentage changes for: A - hepatic total lipids/fat content, B - triglyceride content 19 and C - cholesterol content following lipotrope consumption by rats initially fed steatogen diet 20 (control group). Ranges for duration of the feeding periods and percentages of lipotrope 21 supplementation are presented in Tables 2 and 3. Red crosses and horizontal bars respectively 22 indicate the means and the median. Concerning unsaturated FA, reductions of total/lipid and 23 triglyceride levels have been obtained with arachidonic acid only (Supplemental Table 2). 24 ABBREVIATIONS: HCA, Hydroxycitric Acid; PUFA, Poly-Unsaturated Fatty Acid 
1 Figures 4 A-E. Percentage changes for lipogenic enzyme activities following lipotrope 2 consumption by rats initially fed steatogen diet (control group): A - Fatty Acid Synthase (FAS); B 3 - Malic Enzyme (ME); C - Glucose-6-Phosphate dehydrogenase (G6PDH); D - Acetyl-CoA 4 Carboxylase (ACC), E - ATP-Citrate Lyase/Citrate Cleavage Enzyme (ATP-CL/CCE). Enzymes 5 are those directly involved in FA synthesis, i.e. FAS (Fatty Acid Synthase), ACC (Acetyl-CoA 6 Carboxylase) and ATP-CL/CCE (ATP-Citrate Lyase or Citrate Cleavage Enzyme) and those 7 yielding $\mathrm{NADPH}, \mathrm{H}^{+}$directly used for FA synthesis, i.e. ME (Malic Enzyme) and G6PDH 8 (Glucose-6-Phosphaphate DeHydrogenase). Concerning unsaturated FA, reductions of FAS, ME 9 and G6PDH activities have been obtained with methyl linolenate, methyl linoleate, methyl oleate 10 and ethyl linoleate; and reduction of ACC activity with ethyl linoleate only (Supplemental Table 11 2). ABBREVIATIONS: PUFA, Poly-Unsaturated Fatty Acid 
Table 1 Protective effect of PBF against chronic disease and all-cause mortality risks ${ }^{1}$

All-cause

Weight

Cancers

CVD

Type 2

mortality

control/obesity

Diabetes

\begin{tabular}{|c|c|c|c|c|c|}
\hline Cereals (whole-grain) & + & + & + & + & + \\
\hline Legumes & + & \pm & \pm & \pm & \pm \\
\hline Fruits (not juices) & \pm & + & \pm & + & \pm \\
\hline Vegetables & \pm & + & \pm & + & + \\
\hline Fruits \& Vegetables & + & \pm & + & \pm & \pm \\
\hline
\end{tabular}


Table 2 Lipotropic effects of main plant lipotropes, micronutrients and other compounds on main markers of lipid metabolism in rats

\begin{tabular}{|c|c|c|c|c|c|c|c|c|c|c|c|c|c|}
\hline & & \multicolumn{4}{|c|}{ Main lipotropes } & \multicolumn{3}{|c|}{ Vitamins B } & \multicolumn{5}{|c|}{ Other phytochemicals } \\
\hline & & Choline & Betaine & Myo-inositol & Methionine & Niacin & $\begin{array}{l}\text { Pantothenic } \\
\text { acid }\end{array}$ & Folates & $\mathrm{HCA}$ & Carnitine & Organosulfurs & MUFA/PUFA & Melatonin \\
\hline \multirow[t]{4}{*}{$\mathrm{TL} /$ fat content } & $\mathrm{n}^{\mathrm{a}}$ & 9 & 2 & 6 & 6 & 3 & 1 & 1 & 3 & 7 & 1 & 1 & $-b$ \\
\hline & Duration (days) & $14-65$ & 21 & $13-21$ & $14-65$ & $10-21$ & $16-18$ & 64 & $10-26$ & $7-56$ & 14 & 30 & - \\
\hline & $\%$ of diet & $0.16-0.64$ & $0.16-0.64$ & $0.1-0.515$ & $0.15-0.68$ & $0.2-4$ & $0.001-0.005$ & $\approx 1-25 \mathrm{ppm}^{\mathrm{c}, \mathrm{d}}$ & $-9 /+67$ & $0.1-1.6$ & 0.5 & $\approx 0.1$ & - \\
\hline & Change (range, \%) & $-84 /-39$ & $-79 /-64$ & $-50 / 0$ & $-87 /-10$ & $-46 /-9$ & $-62 /-51$ & $-48 /+11$ & - & $-55 /-7$ & $-11 /-1$ & -63 & - \\
\hline \multirow[t]{4}{*}{ TG content } & $\mathrm{n}$ & 2 & 2 & 8 & - & - & 1 & - & - & 8 & - & 1 & 1 \\
\hline & Duration (days) & $2-3$ & $14-21$ & $3-16.5$ & - & - & $4-21$ & - & - & $7-56$ & - & 30 & 84 \\
\hline & $\%$ of diet & $0.4-0.5$ & 0.5 & $0.1-0.515$ & - & - & 0.01 & - & - & $0.1-1.6$ & - & $\approx 0.1^{\mathrm{c}}$ & $\approx 0.003-0.014^{\circ}$ \\
\hline & Change (range, \%) & $-84 /-60$ & $-62 /-51$ & $-81 /-17$ & - & - & $-79 /-23$ & - & - & $-64 /-4$ & - & -83 & $-17 /-9$ \\
\hline \multirow[t]{4}{*}{ Cholesterol content } & $\mathrm{n}$ & 1 & - & 9 & 1 & - & - & 1 & - & 5 & 2 & - & 1 \\
\hline & Duration (days) & 21 & - & $7-56$ & 42 & - & - & 45 & - & $7-56$ & $14-45$ & - & $30-84$ \\
\hline & $\%$ of diet & 0.2 & - & $0.1-0.515$ & $0.2-0.5$ & - & - & $0.5 \mathrm{ppm}^{\mathrm{d}}$ & - & $0.1-1.6$ & 0.5 & - & $\approx 0.003-0.014^{\circ}$ \\
\hline & Change (range, \%) & $-56 /-52$ & - & $-37 / 0$ & -12 & - & - & $-51 /-6$ & - & $-60 /+16$ & $-21 /-10$ & - & $-28 /-7$ \\
\hline \multirow[t]{4}{*}{ FAS $^{4}$ activity } & $\mathrm{n}$ & 1 & - & 3 & - & - & - & - & - & - & - & 2 & - \\
\hline & Duration (days) & 2 & - & $3-14.5$ & - & - & - & - & - & - & - & $1-7$ & - \\
\hline & $\%$ of diet & 0.4 & - & $0.1-0.5$ & - & - & - & - & - & - & - & $3-5$ & - \\
\hline & Change (range, \%) & -21 & - & $-31 /-29$ & - & - & - & - & - & - & - & $-63 / 0$ & - \\
\hline \multirow[t]{3}{*}{ ME activity } & $\mathrm{n}$ & - & - & 5 & - & - & - & - & - & - & 1 & 1 & - \\
\hline & Duration (days) & - & - & $13-16.5$ & - & - & - & - & - & - & 45 & 7 & - \\
\hline & $\%$ of diet & - & - & $0.1-0.515$ & - & - & - & - & - & - & 0.5 & 3 & - \\
\hline
\end{tabular}




\begin{tabular}{|c|c|c|c|c|c|c|c|c|c|c|c|c|c|}
\hline & Change (range, \%) & - & - & $-42 /-12$ & - & - & - & - & - & - & -10 & $-57 /+3$ & - \\
\hline \multirow[t]{4}{*}{ G6PDH activity } & $\mathrm{n}$ & - & - & 5 & - & - & - & - & - & - & - & 1 & - \\
\hline & Duration (days) & - & - & $13-16.5$ & - & - & - & - & - & - & - & 7 & - \\
\hline & $\%$ of diet & - & - & $0.1-0.515$ & - & - & - & - & - & - & - & 3 & - \\
\hline & Change (range, \%) & - & - & $-43 /-24$ & - & - & - & - & - & - & - & $-69 / 0$ & - \\
\hline \multirow[t]{4}{*}{ ACC activity } & $\mathrm{n}$ & - & - & 1 & - & - & - & - & - & - & - & 1 & - \\
\hline & Duration (days) & - & - & $3-13$ & - & - & - & - & - & - & - & $1-4$ & - \\
\hline & $\%$ of diet & - & - & $0.1-0.5$ & - & - & - & - & - & - & - & 5 & - \\
\hline & Change (range, \%) & - & - & $-31 /-20$ & - & - & - & - & - & - & - & $-57 /-11$ & - \\
\hline \multirow[t]{4}{*}{ ATPCL activity } & $\mathrm{n}$ & - & - & 1 & - & - & - & - & - & - & - & - & - \\
\hline & Duration (days) & - & - & $3-13$ & - & - & - & - & - & - & - & - & - \\
\hline & $\%$ of diet & - & - & $0.1-0.5$ & - & & - & - & - & - & - & - & - \\
\hline & Change (range, \%) & - & - & $-31 /-20$ & - & - & - & - & - & - & - & - & - \\
\hline
\end{tabular}

${ }^{\mathrm{a}}$ Number of references extracted from Supplemental Tables 1 and 2

bo data found

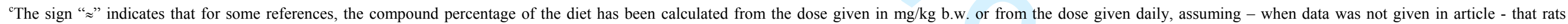
generally consume around $20 \mathrm{~g}$ chow diet daily

${ }^{\mathrm{d}} \mathrm{ppm}=10^{-6}$, i.e. $1 \mathrm{mg} / \mathrm{kg}$

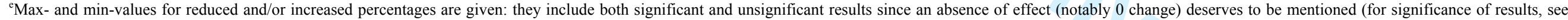
corresponding Supplemental Tables)

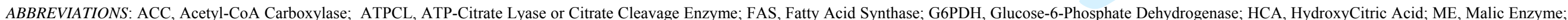
MUFA, Mono-Unsaturated Fatty Acid; PUFA, Poly-Unsaturated Fatty Acid; TG, TriGlyceride; TL, Total Lipids 
Tableau 3 Lipotropic effects of fiber compounds, polyphenols and derived compounds on main markers of lipid metabolism in rats*

\begin{tabular}{|c|c|c|c|c|c|c|c|c|c|c|c|c|}
\hline & & \multicolumn{3}{|c|}{ Fiber-type compounds } & \multicolumn{8}{|c|}{ Polyphenol-type compounds } \\
\hline & & Fiber & Phytic acid & $\begin{array}{l}\text { Oligo- } \\
\text { fructose }\end{array}$ & Phenolic acids & Flavonoids & Lignans & Curcumin & Saponins & Phytosterols & $\gamma$-oryzanol & $\begin{array}{l}\text { Mixture or } \\
\text { plant extract }\end{array}$ \\
\hline \multirow[t]{4}{*}{$\mathrm{TL} /$ fat content } & $\mathrm{n}^{\mathrm{a}}$ & 5 & 5 & 1 & 1 & $-{ }^{b}$ & 2 & 1 & 3 & - & - & - \\
\hline & Duration (days) & $19-63$ & $12-30$ & 42 & 28 & - & 28 & 28 & $14-84$ & - & - & - \\
\hline & $\%$ of diet & $6.5-16$ & $0.1-2.5$ & 10 & 0.4 & - & $0.002-0.2$ & 0.2 & $0.001-0.07$ & - & - & - \\
\hline & Change (range, $\%)^{\mathrm{c}}$ & $-60 /+12$ & $-52 /-29$ & -43 & -9 & - & $-24 /+7$ & -4 & $-45 /+8$ & - & - & - \\
\hline \multirow[t]{4}{*}{ TG content } & $\mathrm{n}$ & 6 & 5 & 6 & 1 & 4 & 4 & 1 & 2 & 2 & 2 & 3 \\
\hline & Duration (days) & $28-56$ & $12-30$ & $19-70$ & 49 & $28-42$ & $10-15$ & 49 & $21-84$ & $31-35$ & 49 & $35-63$ \\
\hline & $\%$ of diet & $3-10$ & $0.1-2.5$ & $5-10$ & 0.075 & $0.1-1$ & $0.06-0.5$ & 0.15 & $\approx 0.005-1^{\mathrm{d}}$ & $0.1-2$ & $0.2-1.2$ & $\approx 0.15-2.5^{\mathrm{d}, \mathrm{e}}$ \\
\hline & Change (range, \%) & $-85 /+47$ & $-84 /-42$ & $-57 /-1$ & -19 & $-23 /+3$ & $-68 /+136$ & -22 & $-40 /-35$ & $-12 /+16$ & $-33 /-7$ & $-27 /+35$ \\
\hline \multirow[t]{4}{*}{ Cholesterol content } & $\mathrm{n}$ & 14 & 4 & 3 & 2 & 4 & 5 & 2 & 3 & 4 & 3 & 3 \\
\hline & Duration (days) & $9-63$ & $13-30$ & $19-56$ & $28-49$ & $28-42$ & $10-28$ & $28-49$ & $19-84$ & $13-35$ & $28-49$ & $35-63$ \\
\hline & $\%$ of diet & $0.6-30$ & $0.5-1.02$ & $5-10$ & $0.075-0.4$ & $0.1-1$ & $0.06-0.5$ & $0.15-0.2$ & $\approx 0.005-1^{\mathrm{d}}$ & $0.1-5$ & $0.01-1.2$ & $\approx 0.15-0.61^{\mathrm{d}}$ \\
\hline & Change (range, \%) & $-75 /+23$ & $-13 / 0$ & $-14 /-3$ & $-3 /+1$ & $-28 /+14$ & $-39 /+21$ & $-37 /-16$ & $-52 /+14$ & $-76 /-18$ & $-26 /-14$ & $-19 /-7$ \\
\hline \multirow[t]{4}{*}{ FAS $^{5}$ activity } & $\mathrm{n}$ & - & 2 & 4 & - & 1 & 6 & - & - & - & - & - \\
\hline & Duration (days) & - & $12-13$ & $21-70$ & - & 182 & $10-15$ & - & - & - & - & - \\
\hline & $\%$ of diet & - & $0.1-2.5$ & 10 & - & $\approx 0.0018^{\mathrm{d}}$ & $0.06-0.5$ & - & - & - & - & - \\
\hline & Change (range, \%) & - & $-65 /-26$ & $-41 / 0$ & - & 0 & $-63 /-21$ & - & - & - & - & - \\
\hline \multirow[t]{3}{*}{ ME activity } & $\mathrm{n}$ & - & 5 & 2 & - & - & 2 & - & - & - & - & - \\
\hline & Duration (days) & - & $12-13$ & $42-70$ & - & - & 15 & - & - & - & - & - \\
\hline & $\%$ of diet & - & $0.1-2.5$ & 10 & - & - & $0.1-0.5$ & - & - & - & - & - \\
\hline
\end{tabular}




\begin{tabular}{|c|c|c|c|c|c|c|c|c|c|c|c|c|}
\hline & Change (range, \%) & - & $-44 /-2$ & $-16 / 0$ & - & - & $-50 /+125$ & - & - & - & - & - \\
\hline \multirow[t]{4}{*}{ G6PDH activity } & $\mathrm{n}$ & - & 5 & - & - & - & 5 & - & - & - & - & - \\
\hline & Duration (days) & - & $12-13$ & - & - & - & $10-15$ & - & - & - & - & - \\
\hline & $\%$ of diet & - & $0.1-2.5$ & - & - & - & $0.06-0.4$ & - & - & - & - & - \\
\hline & Change (range, \%) & - & $-47 /+5$ & - & - & - & $-77 /-3$ & - & - & - & - & - \\
\hline \multirow[t]{4}{*}{ ACC activity } & $\mathrm{n}$ & - & 1 & - & - & 1 & 2 & - & - & - & - & - \\
\hline & Duration (days) & - & 13 & - & - & 182 & $15-28$ & - & - & - & - & - \\
\hline & $\%$ of diet & - & 0.5 & - & - & $\approx 0.0018$ & $0.1-0.4$ & - & - & - & - & - \\
\hline & Change (range, \%) & - & -16 & - & - & 0 & $-57 /-36$ & - & - & - & - & - \\
\hline \multirow[t]{4}{*}{ ATPCL activity } & $\mathrm{n}$ & - & 1 & 2 & - & - & 5 & - & - & - & - & - \\
\hline & Duration (days) & - & 13 & $42-70$ & - & - & $10-15$ & - & - & - & - & - \\
\hline & $\%$ of diet & - & 0.5 & 10 & - & - & $0.06-0.4$ & - & - & - & - & - \\
\hline & Change (range, \%) & - & -37 & $-26 / 0$ & - & - & $-70 /-30$ & - & - & - & - & - \\
\hline \multirow[t]{4}{*}{$\operatorname{PPAR} \alpha$ mRNA level } & $\mathrm{n}$ & - & - & - & - & 1 & & - & - & - & - & - \\
\hline & Duration (days) & - & - & - & - & 182 & - & - & - & - & - & - \\
\hline & $\%$ of diet & - & - & - & - & $\approx 0.0018^{\mathrm{d}}$ & - & - & - & - & - & - \\
\hline & Change (range, \%) & - & - & - & - & +160 & - & - & - & - & - & - \\
\hline \multirow[t]{4}{*}{ SREBP mRNA level } & $\mathrm{n}$ & - & - & - & - & - & 2 & - & - & - & - & - \\
\hline & Duration (days) & - & - & - & - & - & $14-28$ & - & - & - & - & - \\
\hline & $\%$ of diet & - & - & - & - & - & $0.002-0.4$ & - & - & - & - & - \\
\hline & Change (range, \%) & - & - & - & - & - & $-55 /-9$ & - & - & - & - & - \\
\hline
\end{tabular}

${ }^{\mathrm{a}}$ Number of references extracted from Supplemental Tables 3 and 4

${ }^{b}$ No data found 


\section{Page 155 of 267}

\section{Critical Reviews in Food Science and Nutrition}

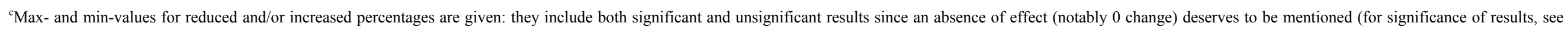
corresponding Supplemental Tables)

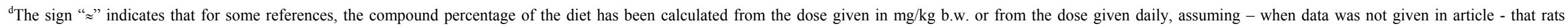
generally consume around $20 \mathrm{~g}$ chow diet daily

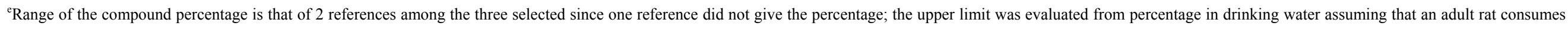
around $20 \mathrm{~mL}$ water daily

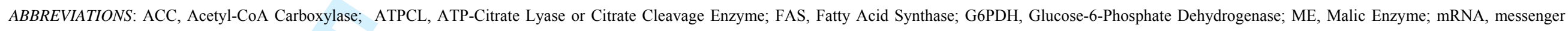
RiboNucleic Acid; PPAR $\alpha$, Peroxisone Proliferator Activated Receptor alpha; SREBP, Sterol Regulatory Element-Binding Proteins; TG, TriGlyceride; TL, Total Lipids 


\section{Figure 1}

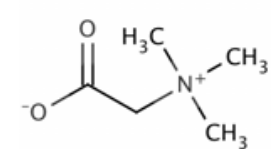

Betaine

$\mathrm{Mg}^{2+}$

Magnesium

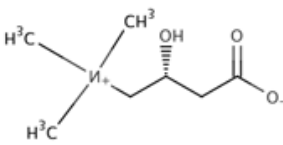

Carnitine

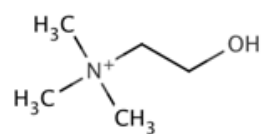

Choline

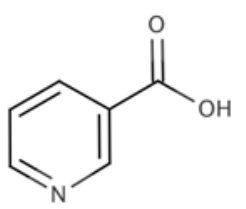

Niacin

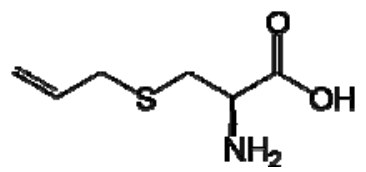

s-allyl cysteine (organosulfur compound)

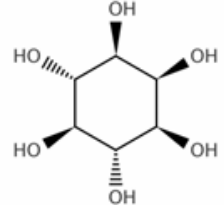

Myo-inositol

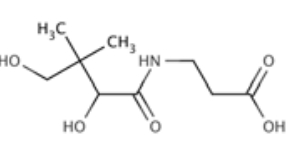

Pantothenic acid

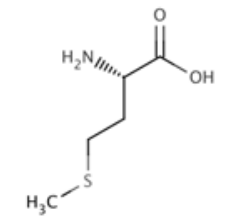

Methionine

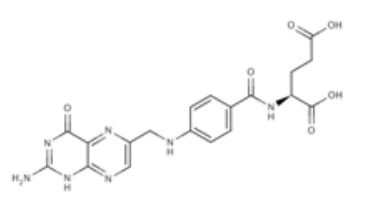

Folates

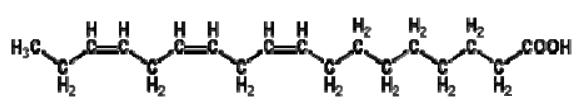

Acide $\alpha$-linolenic (PUFA)

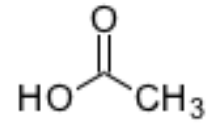

Acetic acid (SCFA) 


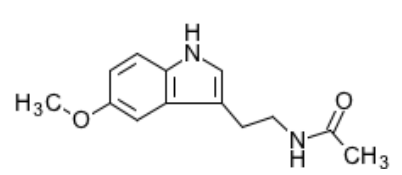

Melatonin

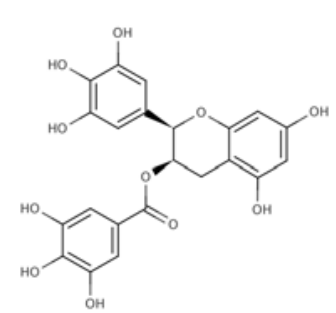

Epigallocatechin gallate (flavonoid)

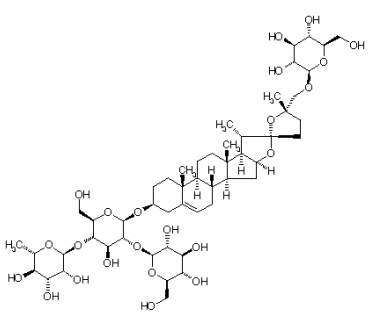

Avenacoside A (saponin)

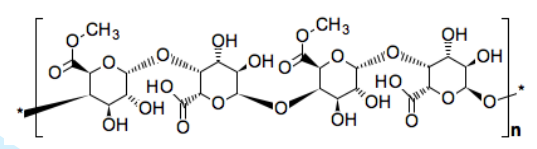

Pectin (soluble fiber)

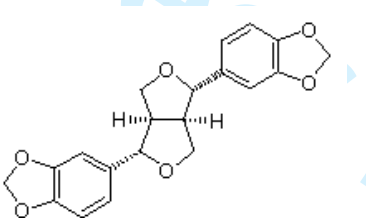

Sesamin (lignan)

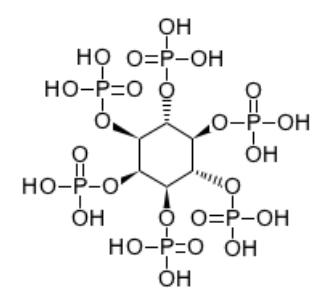

Phytic acid

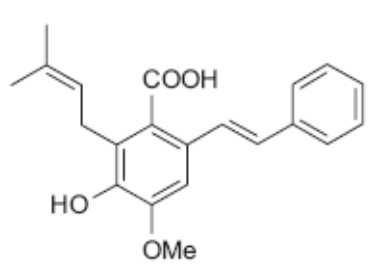

Cajanin (stilbene)

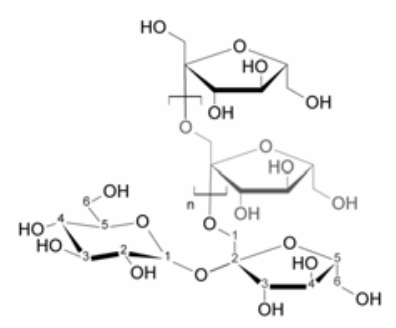

Fructans (oligofructose)

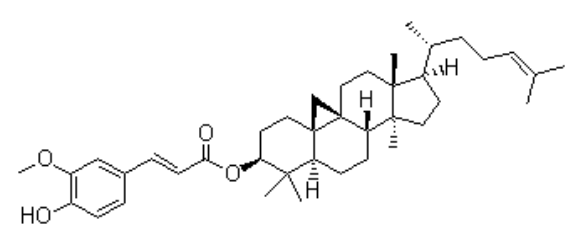

$\gamma$-oryzanol
1-Deoxynojirimycin

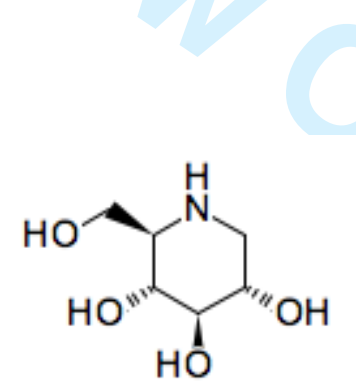

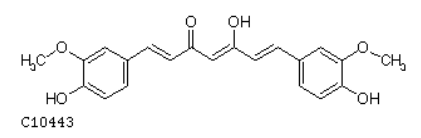

Curcumin 
Figure 2 A-D

A)

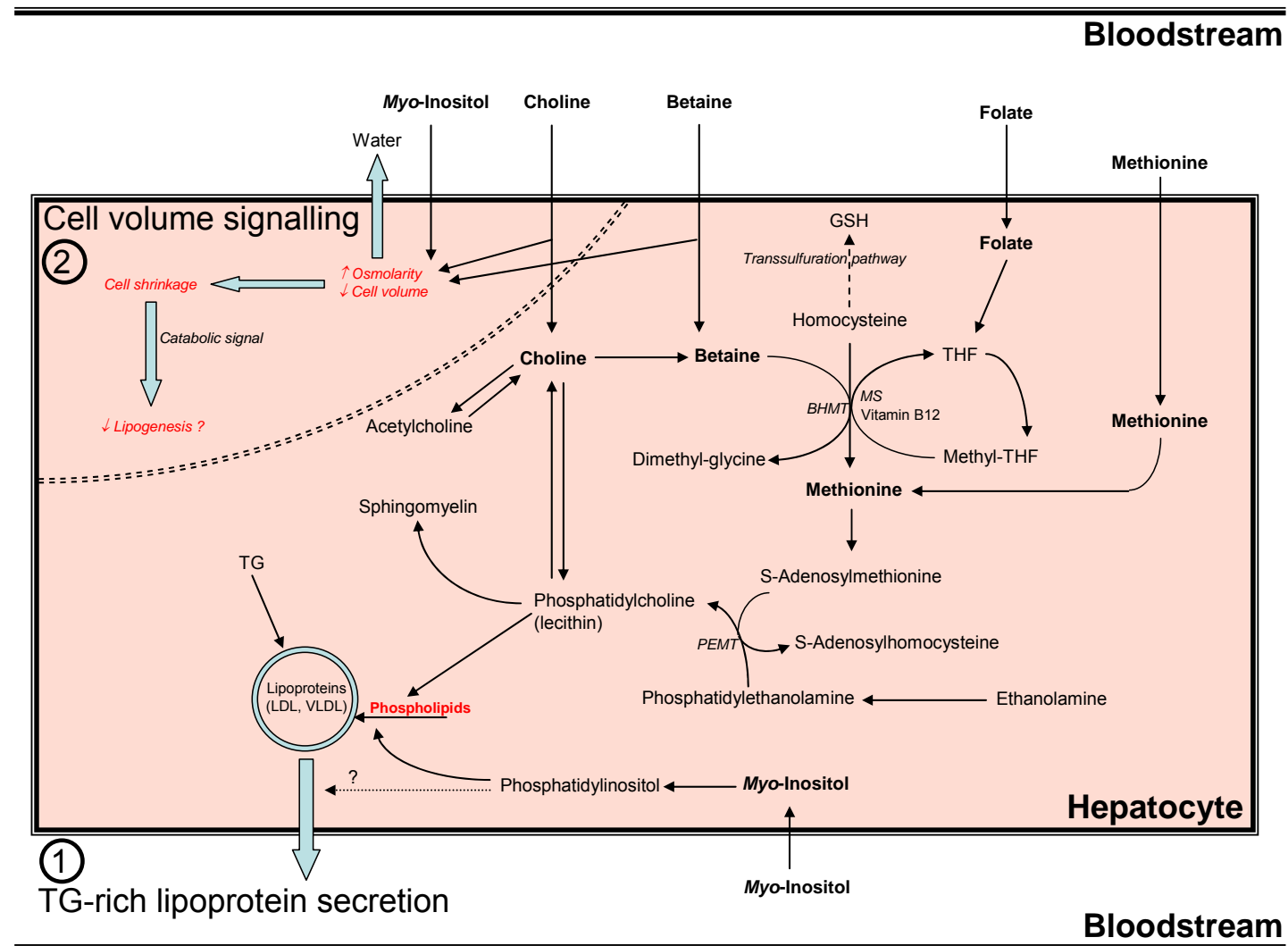

B)

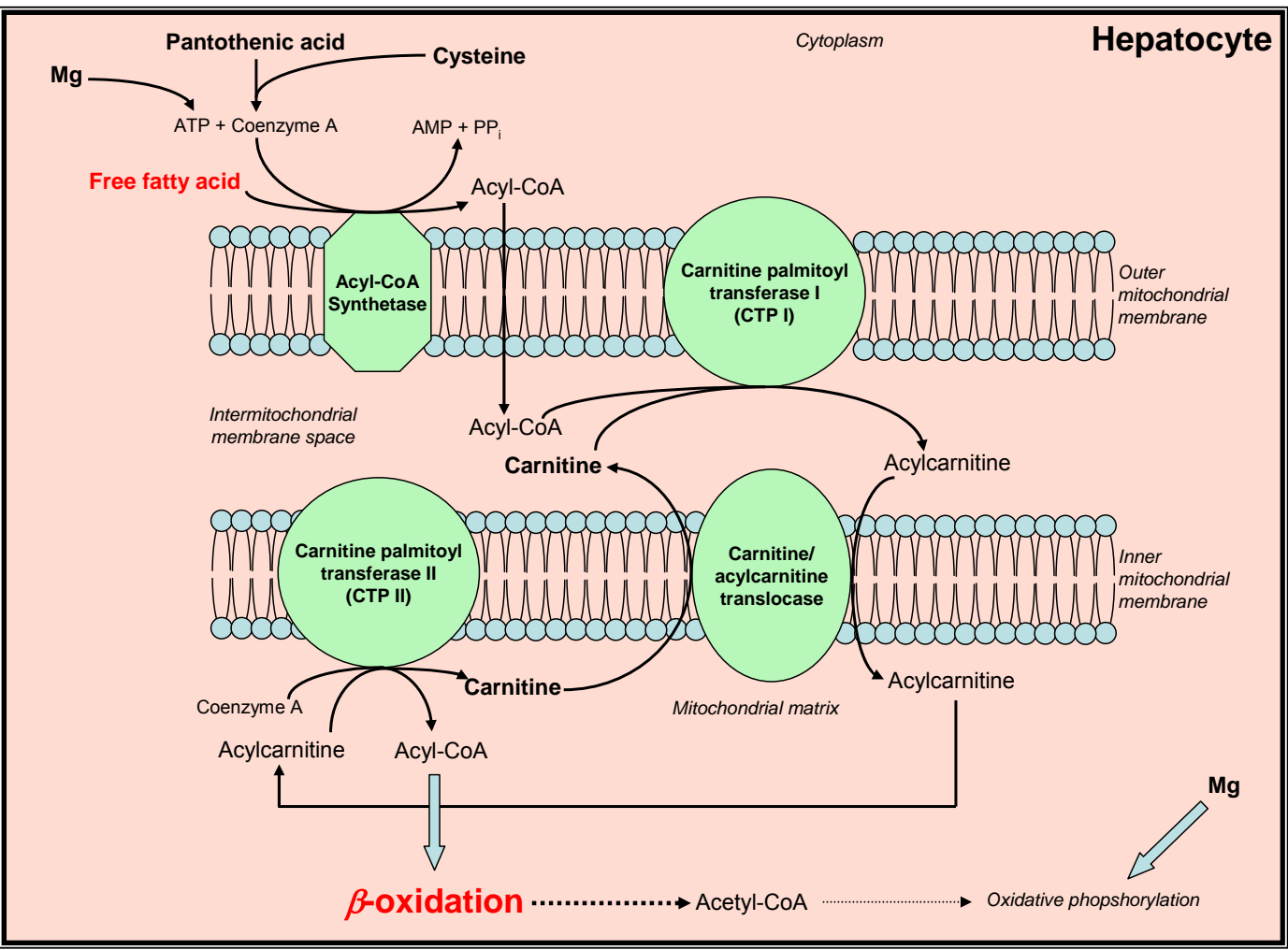


C)

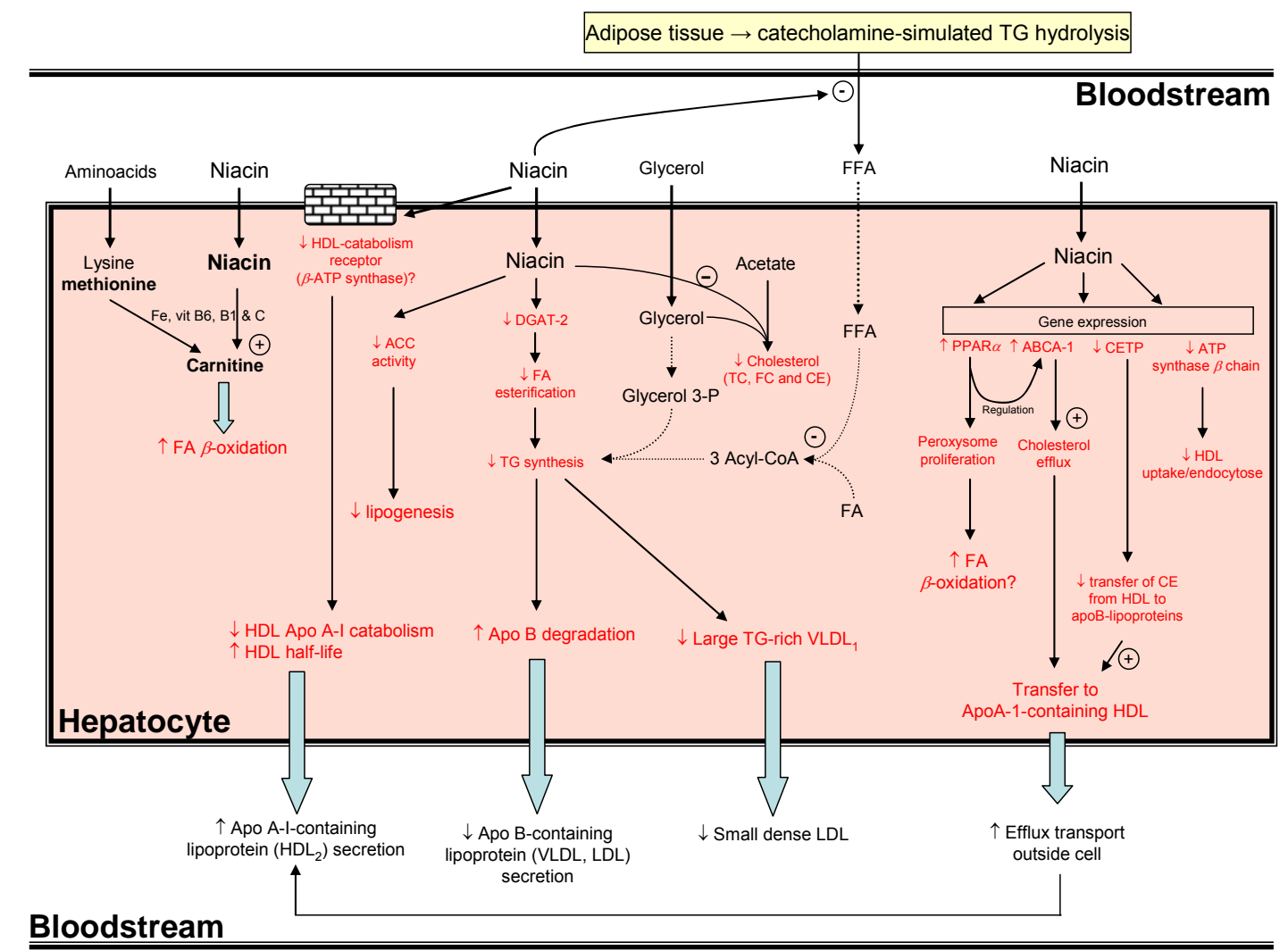

D)

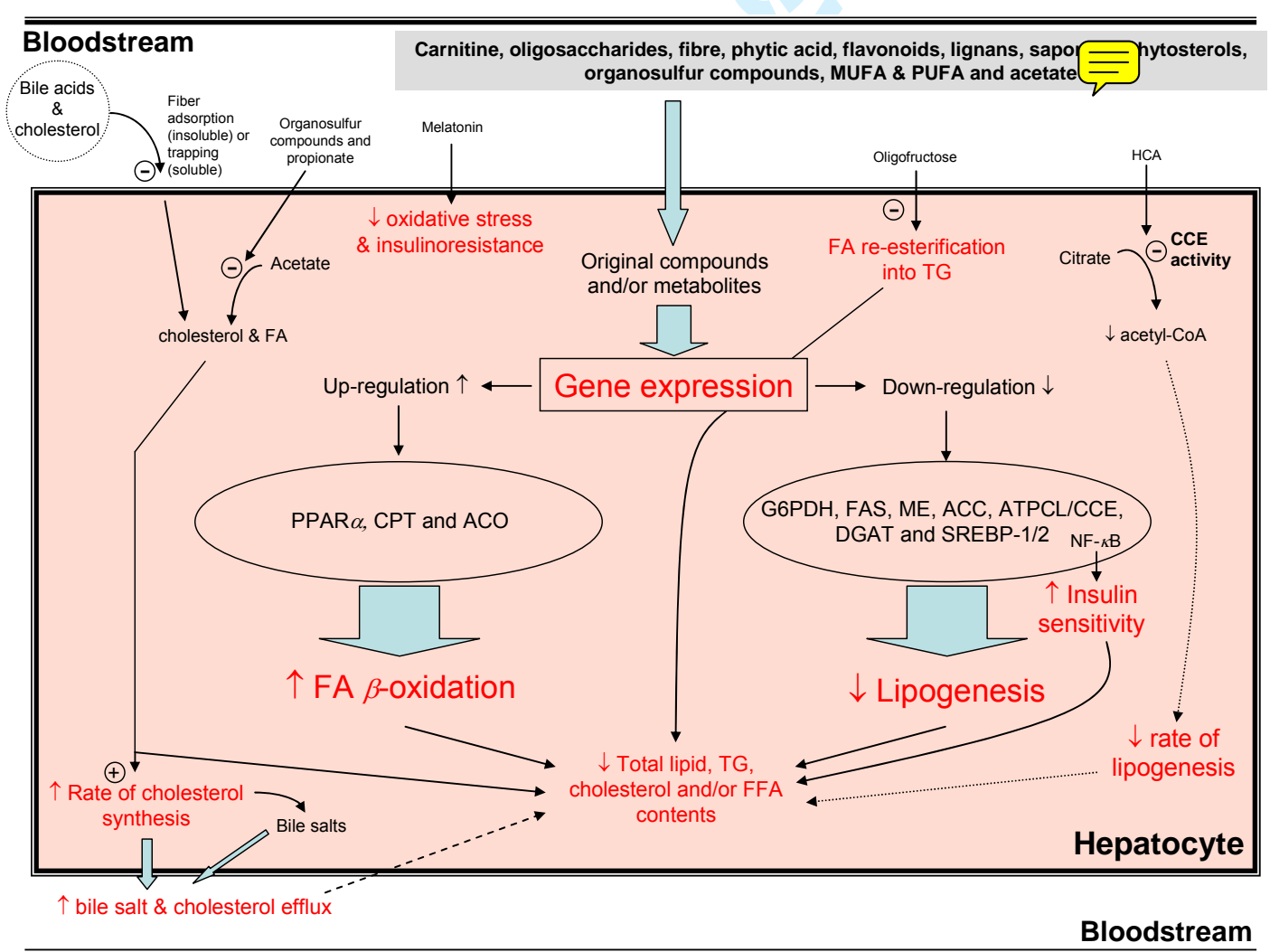


Figure 3 A-C $\equiv$

$\mathrm{TL}$

A)

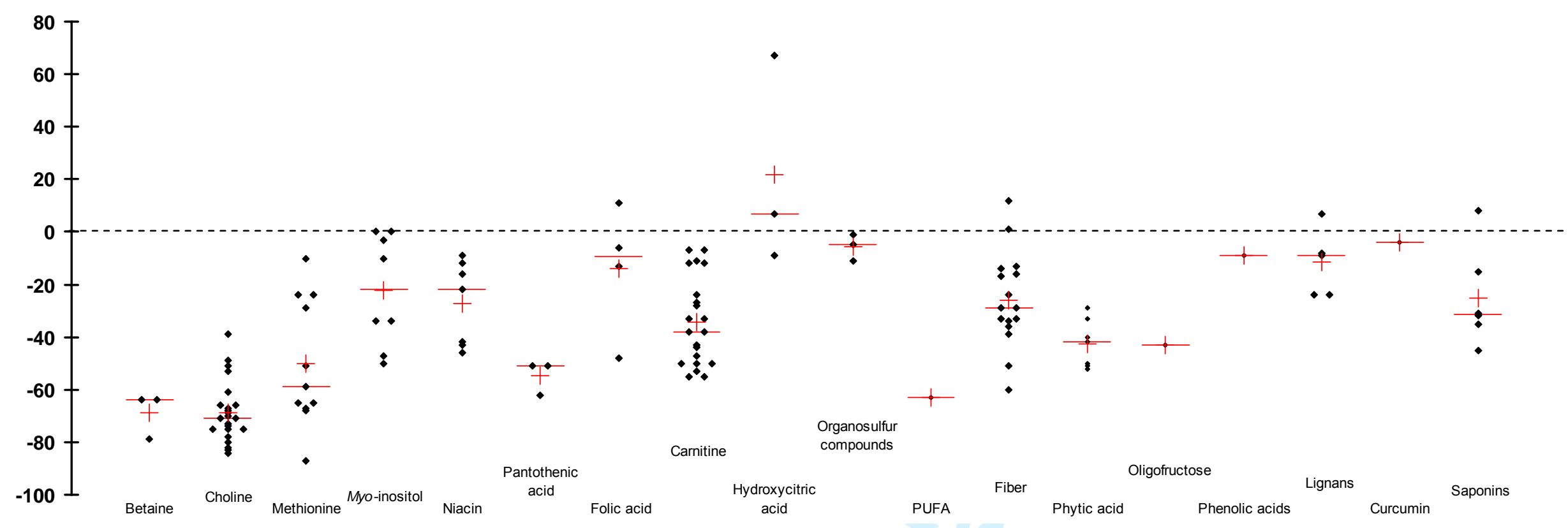

Retirer valeurs de Schön (1958) et les mettre dans Total Cholesterol. 
B) TG: vérifier pour resistant starch si valeurs à ajouter

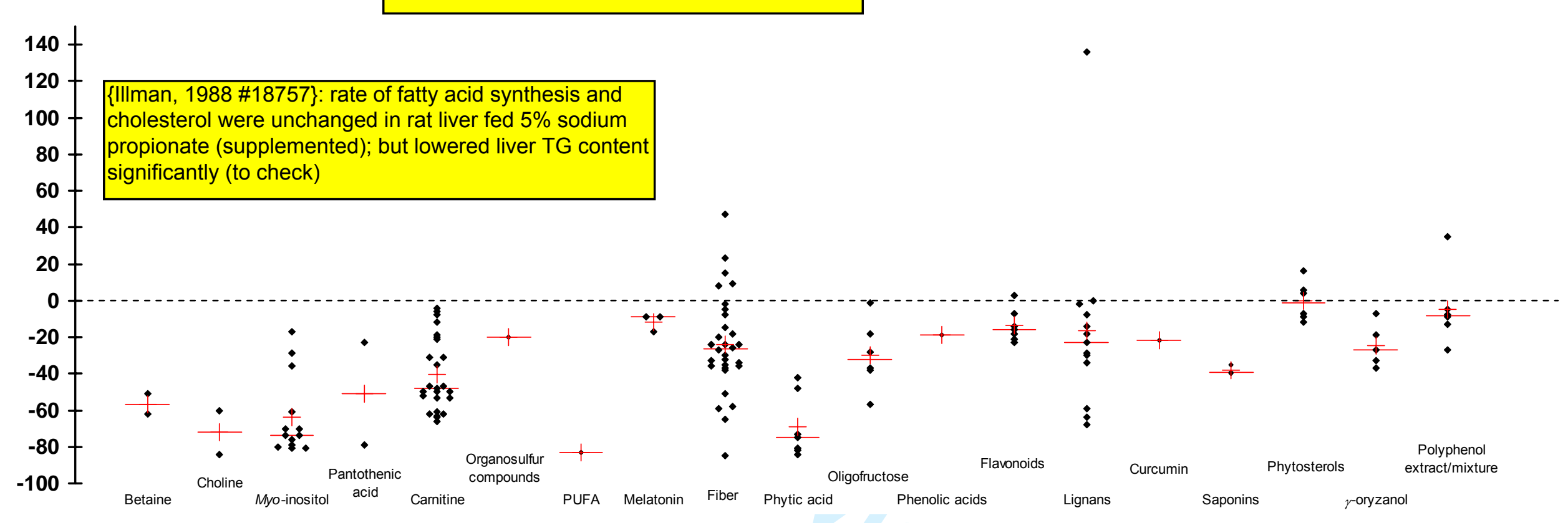


C)

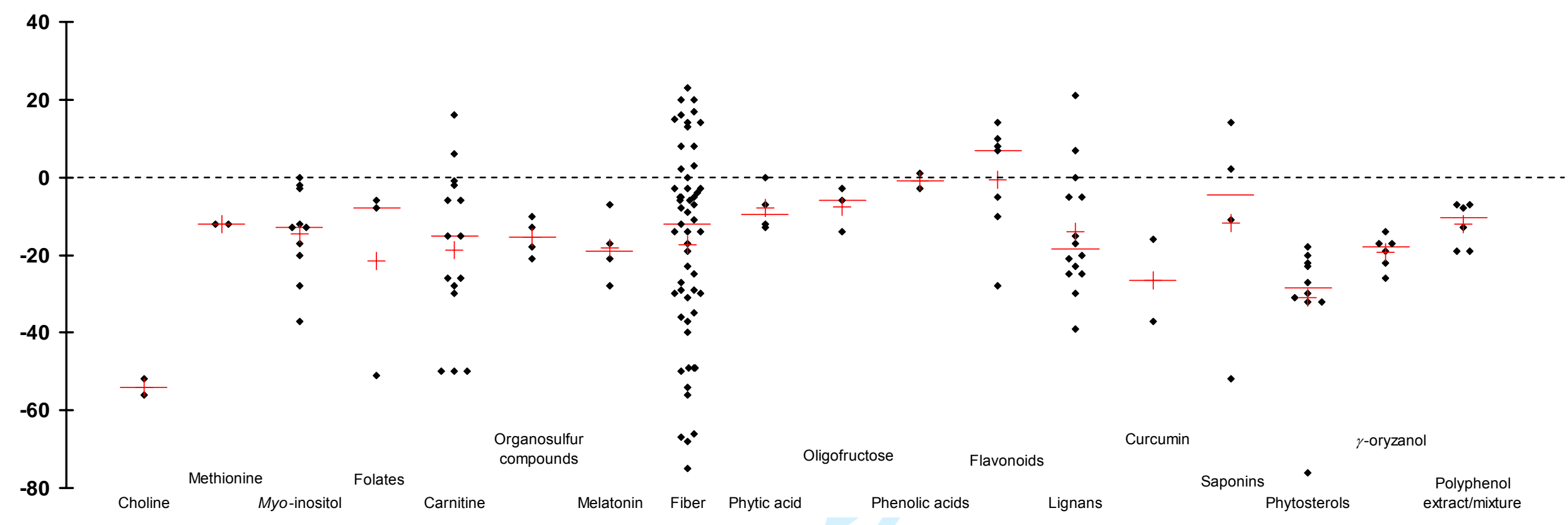

Ajouter valeurs de Schön (1958) pour Total Cholesterol / Niacine 
Figure 4 A-E

A)

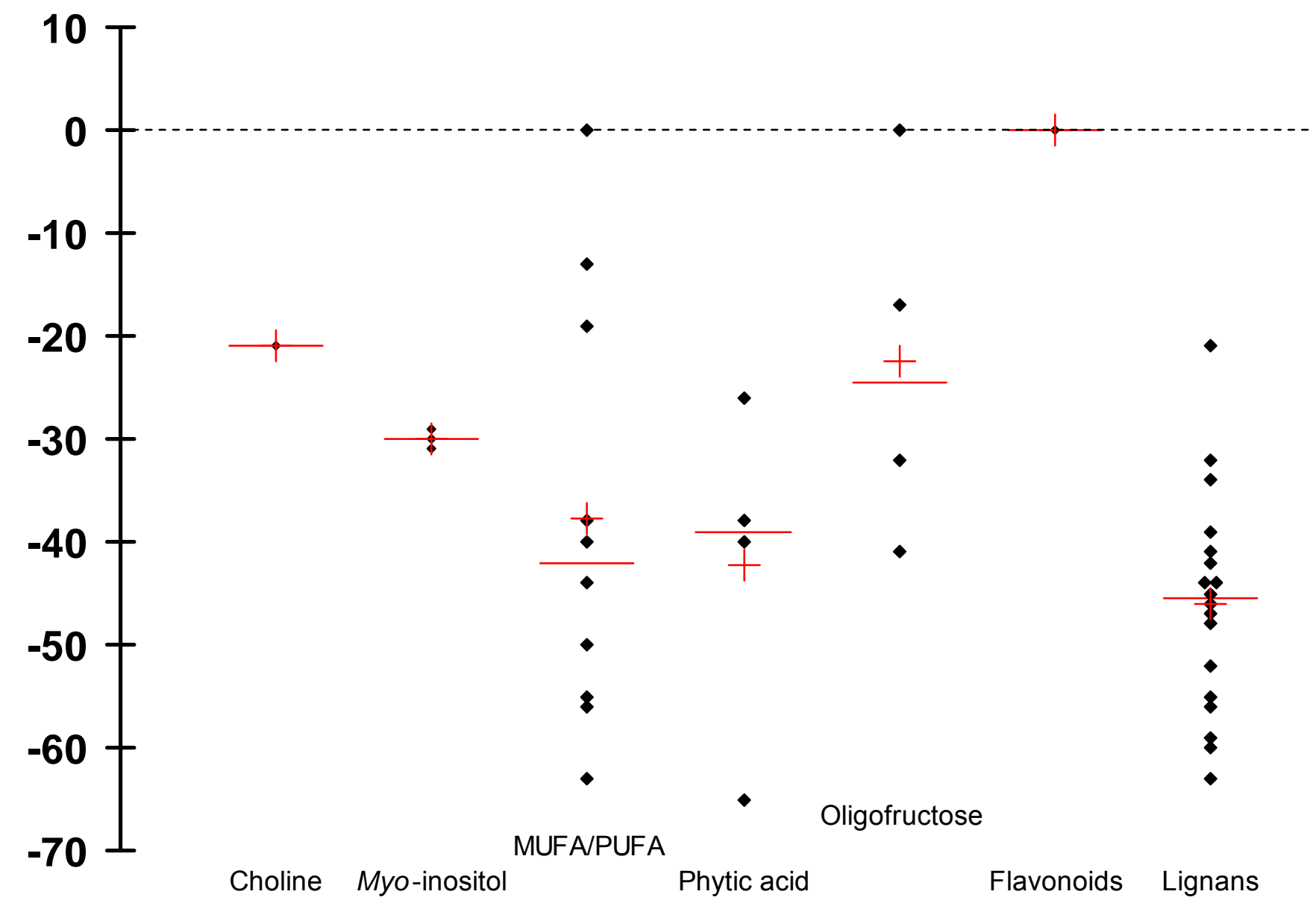


B)

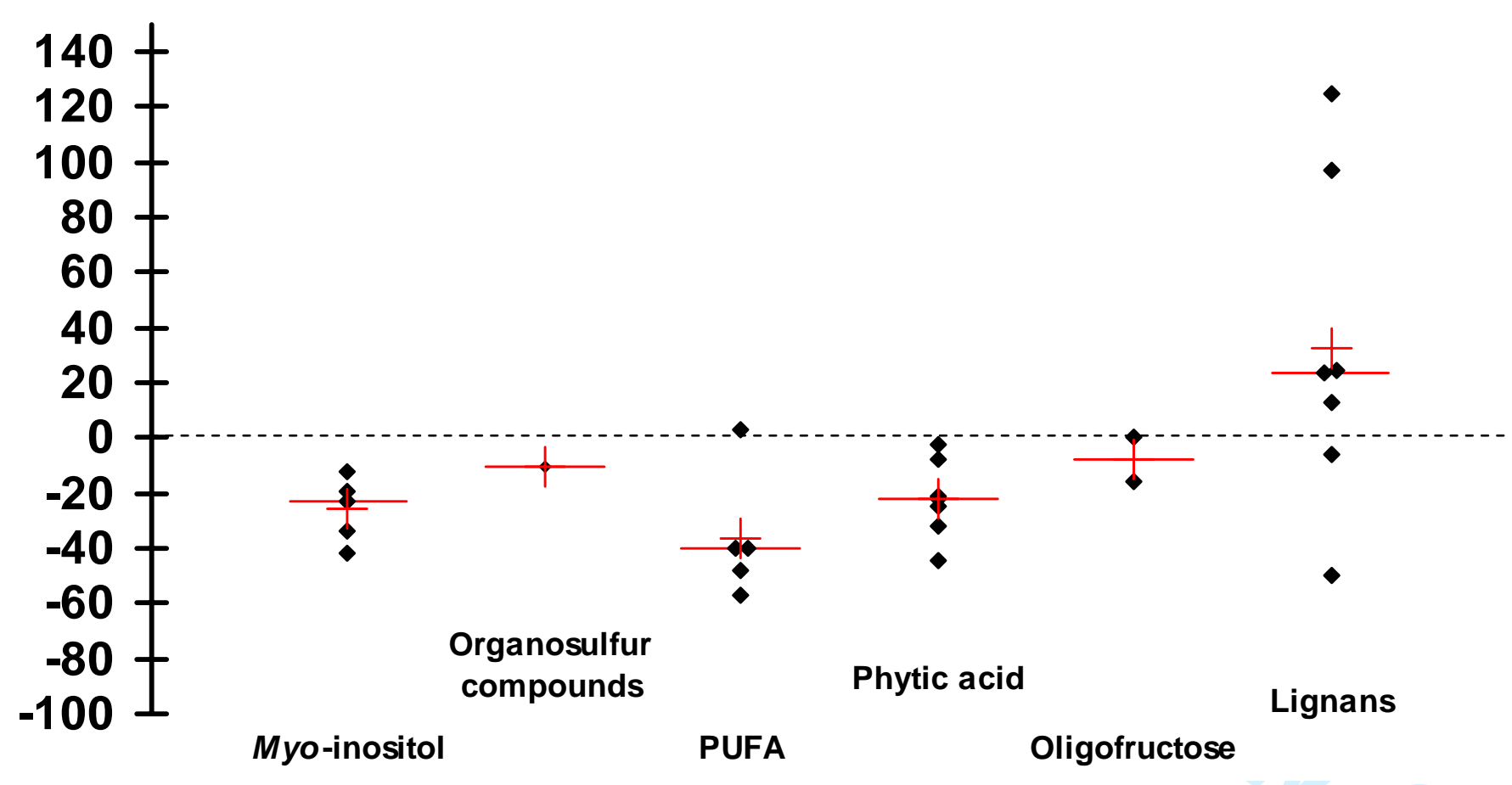


C)
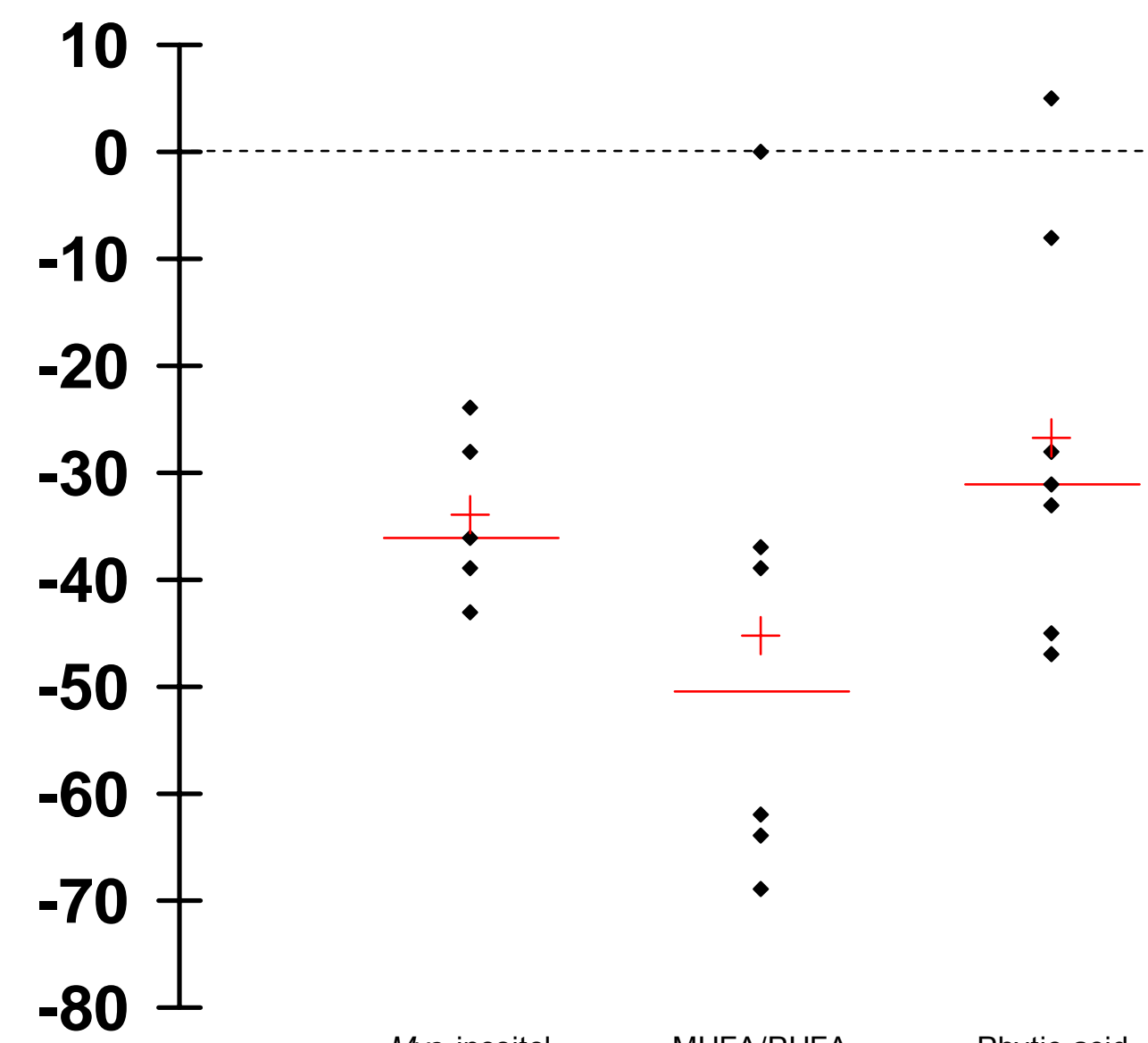

Myo-inosito

MUFA/PUFA

Phytic acid

Lignans 
D)

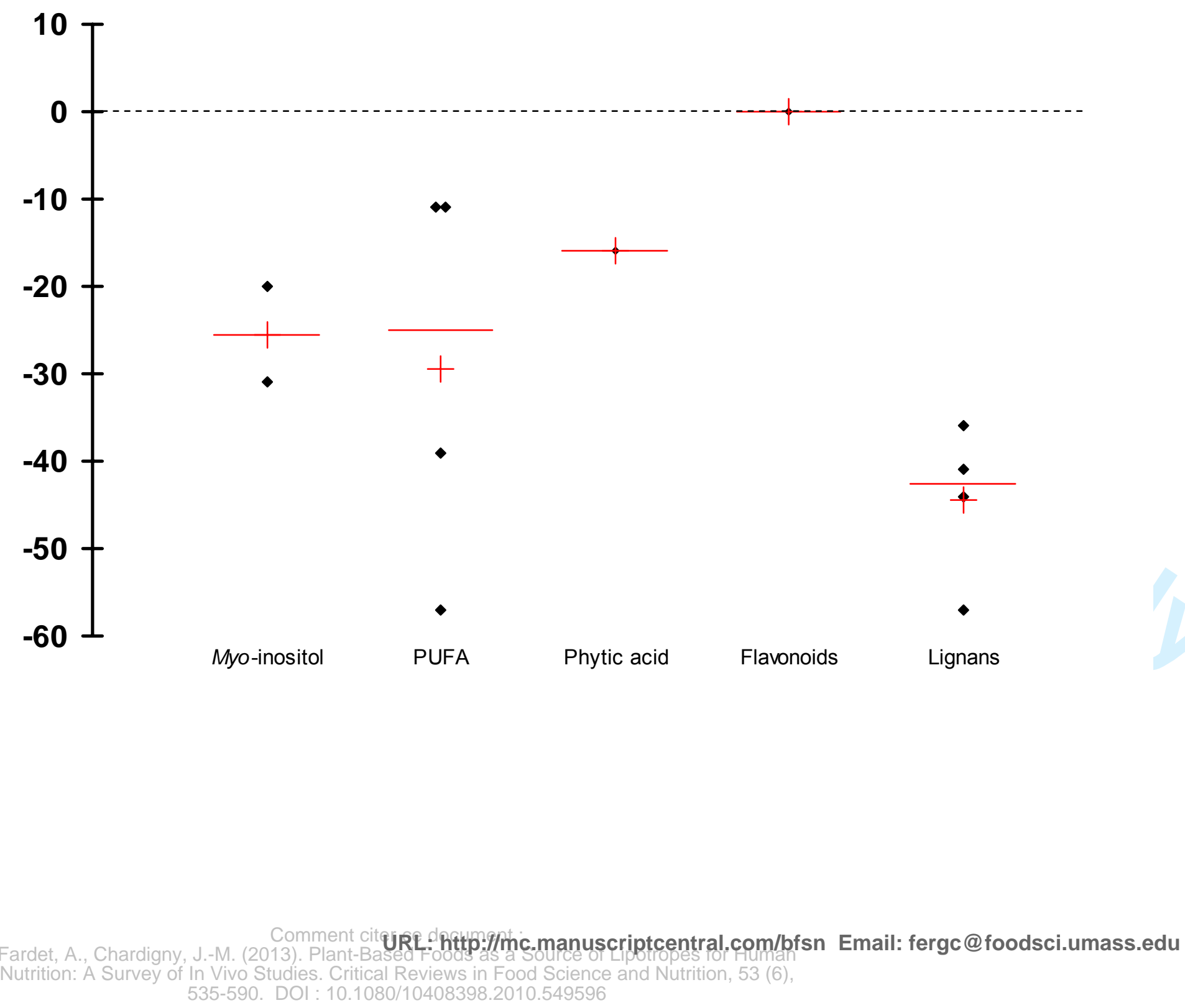


E)

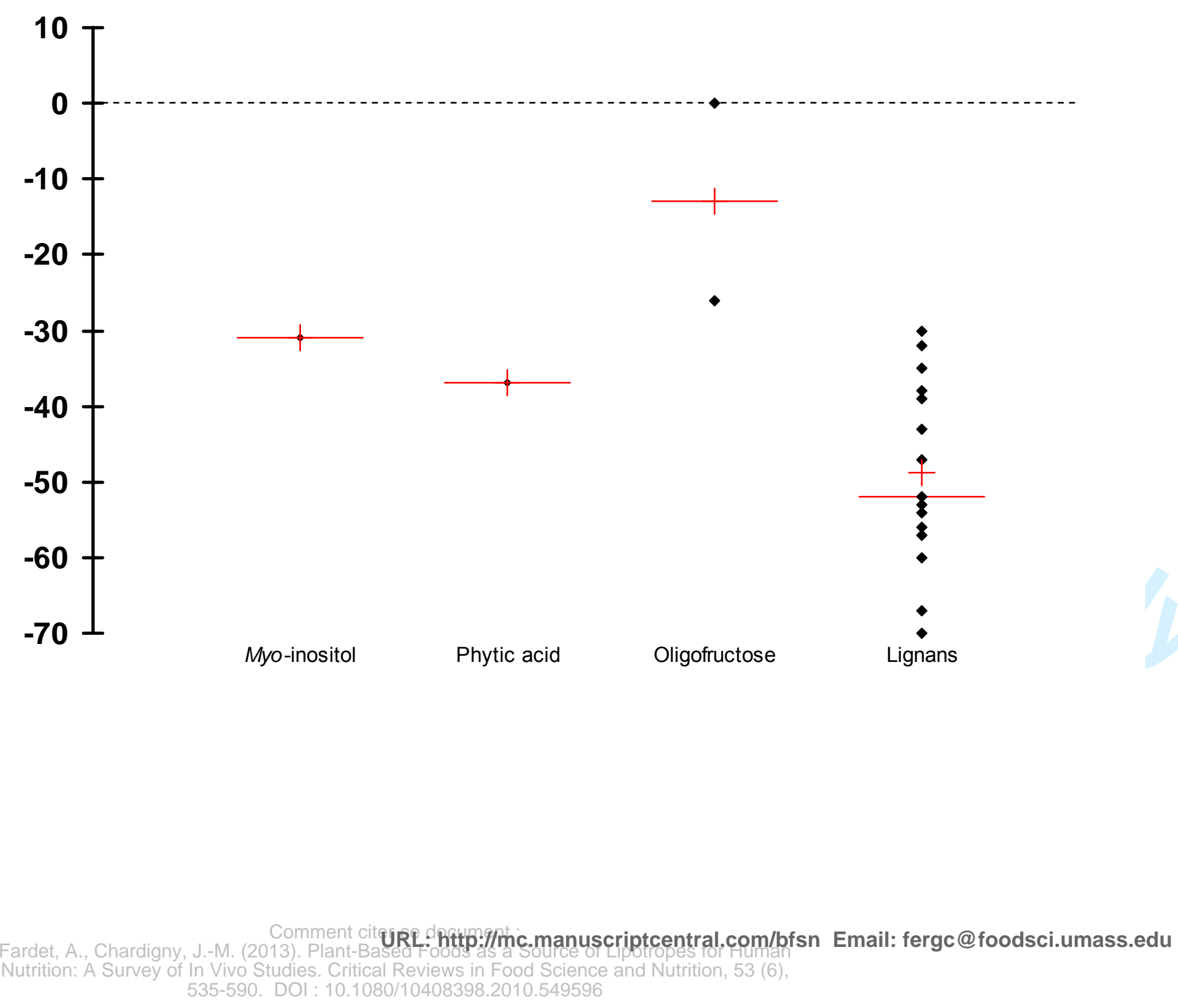


9 A - Main lipotropes

11 A1 - Betaine

12

13 Betaine

14 Betaine
15 Betaine

16 hydrochloride

17

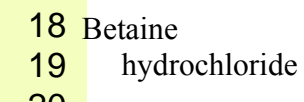

21

22 Betaine

24 Betaine aspartate

26 Anhydrous betaine

41 Betaine 43 A2 - Choline

45 Choline

46
47

47 Choline -in humans \{Elias, $2010 \# 25149\}$ see in animals

Rats fed fat-free and methionine-restricted diet

lipotrope
exposition

\section{Rats fed high-fat $(40 \%)$ diet
Rats fed high-fat $(40 \%)$ diet}

Rats fed high-fat $(40 \%)$ diet

Rats fed high-fat $(20 \%)$ diet
$(48.9 \%$

Rats fed high-fat $(20 \%)$ and high-sucrose

$(48.9 \%)$ diet added with $0.3 \%$ cystine

Rats fed high-fat $(30 \%)$ and methionine-

Rats fed high-sucrose $(45.8 \%)$ and betaine-

deficient diet supplemented with histidine,

lysine and threonine
Rats fed high-fat $(40 \%)$ diet

Rats fed semiliquid ethanol diet

Rats fed ethanol diet

Humans with NASH

$\begin{array}{lll}120 \mathrm{mg} & 21 \text { days } & \downarrow \text { FA percentage }(-59 \%)^{b} \\ 100 \mathrm{mg} & 21 \text { days } & \downarrow \text { FA percentage }(-82 \%)\end{array}$

From 100 to $200 \quad 8$ days $\quad \downarrow$ fat percentage $(-51 \%)$

$\mathrm{mg}$
From 50 to $200 \quad 8$ days $\quad \downarrow$ fat percentage $(-54 \%)$

$\begin{array}{ll}\mathrm{mg} \\ 0.16 \% \text { free } & 21 \text { days }\end{array}$

betaine
$0.32 \%$ free $\quad 21$ days

betaine
From 0.08 to $\quad 21$ days

$0.64 \%$

$250 \mathrm{mg}$ free $\quad 30$ days

betaine $/ \mathrm{kg}$ bw
$0.5 \%$ of diet

$0.5 \%$ of diet $\quad 21$ day

$20 \mathrm{~g}$ solution 1 year

Rats fed low-protein (14.7\%)/low-fat $(\approx 3 \%)$ diet $\quad \begin{aligned} & \text { daily } \\ & 1,2 \text { or } 5 \%\end{aligned} 28$ days (BIBRA diet) \pm betaine for 28 days then the

same diet without betaine for 28 days

Rats fed balanced diet $(\approx 8 \%$ fat and $23.5 \%$ protein; Brandeis University diet)
Intragastric alcohol-fed mice

$0.5,0.75,1.0$ or $\quad 28$ days

0.5 or $1.5 \%$ of $\quad 28$ days

Intragastric alcohol-fed mice

0.5 or $1.5 \%$

28 days

$\begin{array}{lll}\text { Inanol-treated guinea pigs for the last } 10 \text { days } & 2 \% \text { of diet } & 30 \text { days } \\ \text { lsolated hepatocytes from ethanol-fed rats for } 4 & 1 \mathrm{mM} & 4 \mathrm{hrs}\end{array}$

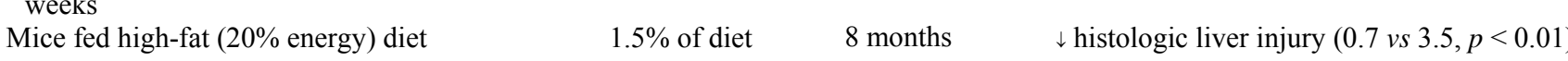

Mice fed high-fat ( $20 \%$ energy) diet $\quad 1.5 \%$ of diet $\quad 8$ months $\quad \downarrow$ histologic liver injury $(0.7$ vs $3.5, p<0.01)$

$\downarrow$ TL percentage $(z-64 \%)$

$\downarrow$ TL percentage $(\approx-64 \%)$

$\downarrow$ TL percentage (from 0 to $-79 \%)$ : sharp decrease begins at a level $\quad$ (Young et al., 1965)
of $0.16 \%$ betaine $\mathrm{HCl}$ supplementation $(-42 \%)$

$\mathrm{C}^{14}$-trioleine cotabolism $\left(-44^{\circ}\right.$ trioleine

$\downarrow$ TG content $(-62 \%), \uparrow$ SAM $(+354 \%)$ and betaine $(+305 \%) \quad$ (Barak et al., 1996)

concentrations and $\uparrow$ BHMT activity $(+46 \%)$
$\downarrow$ TG content $(-51 \%), \uparrow$ SAM concentrations $(+722 \%)$ and $\uparrow$ BHMT $\quad$ (Barak et al., 1997)

activity $(+92 \%)$
Improvement in degree of steatosis, necroinflammatory grade and (Abdelmalek et al., 2001)

stage of fibrosis, $\downarrow$ ALT and AST concentrations (-69\%)
Liver histology: $\uparrow$ lipid droplet and microvacuolisation upon

betaine treatment (resp. $+45,+90$ and $+125 \%$ ), then $\downarrow$
microvacuolisation upon the last 28 datys without betaine (resp.

$-62,-59$ and $-71 \%$ )
$\downarrow$ TG content (resp. - $11 \%$, NS, $-20 \%$, NS, $-13 \%$, NS, and $-39 \%$ )

$\downarrow$ cholesterol $(-18$ and $-47 \%)$ and TG $(-29$ and $-67 \%)$ levels, $\downarrow$ SREBP-1 relative mRNA expression $(\approx-50$ and $z-70 \%)$

$\downarrow$ TG level $(-43 \%)$

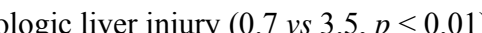

ayes et al., 2003)

$\{\mathrm{di}, 2007$ \#21172\}: "Role of the

blunt and betaine system in

alcoholic and non-alcoholic

hyperhomocysteinemia and

(Ji and Kaplowitz, 2003)

(Balkan et al., 2004)

(Kharbanda et al., 2005)

(Borgschulte et al., 2008)

er steatosis" in BHMT

transgenic mice

\begin{abstract}
Rats fed high-fat (40\%) diet
\end{abstract}
Rats fed high-fat $(40 \%)$ diet

Rats fed high-fat $(40 \%)$ die

Rats fed high-fat $(20 \%)$ and high-sucrose

(48.9\%) diet

Rats fed high-fat $(20 \%)$ and high-sucrose

$(48.9 \%)$ diet added with $0.3 \%$ cystine
Patients $(n=10)$ with decompensted portat $\begin{array}{ll}70 \mathrm{mg} & 21 \text { days } \\ \text { From } 10 \text { to } 117 & 21 \text { days }\end{array}$

$\begin{array}{rl}\mathrm{mg} & 21 \text { days } \\ 75 \mathrm{mg} & 21 \text { days }\end{array}$

$\begin{array}{ll}70 \mathrm{mg} & 21 \text { days } \\ \text { From } 20 \text { to } 40 \quad 8 \text { days }\end{array}$

From 15 to $75 \quad 8$ days

$\mathrm{mg}$
$0.5 \mathrm{~g}$ thrice $\downarrow$ FA percentage $(-64 \%)$

FA percentage (from -40 to -69\%)

$\downarrow$ FA percentage $(-68 \%)$

$\downarrow$ fat percentage $(-37 \%)$

$\downarrow$ fat percentage $(-60 \%)$

Case 2: complete disappearance of ascites and smaller liver
(Best and Huntsman, 1932)

(Best, 1934)

(Best and Huntsman, 1935)

(Russakoff and Blumberg, 1944) 


\begin{tabular}{|c|c|c|c|c|c|}
\hline 2 & cirrhosis of the liver (cirrhosis is frequently & $0.5 \mathrm{~g} 4$ times & 3 weeks & Case 3: complete disappearance of ascites, improved liver function & \\
\hline 3 & $\begin{array}{l}\text { associated with extensive fatty infiltration of } \\
\text { the liver) and treated with a high protein, }\end{array}$ & $4.5 \mathrm{~g}$ & $\approx 9$ months & $\begin{array}{l}\text { tests, feeling of well-being and good health } \\
\text { Case 5: marked improvement (e.g. } \downarrow \text { ascites) }\end{array}$ & \\
\hline 5 & high carbohydrate and low fat diet & $6 \mathrm{~g}$ then $4.5 \mathrm{~g}$ & $\approx 6$ months & Case 7: improvements (e.g. less abdominal paracenteses required) & \\
\hline $\begin{array}{l}6 \\
7\end{array}$ & & & then 6 months & & \\
\hline 8 & & $\begin{array}{l}4-6 \mathrm{~g} \\
1.5 \mathrm{~g} \text { thrice }\end{array}$ & $\begin{array}{l}45 \text { days } \\
4 \text { weeks }\end{array}$ & 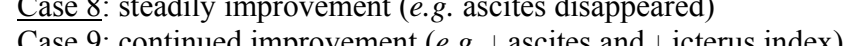 & \\
\hline 9 & & & $\geq 10$ days & Case 10: considerable improvements (e.g. $\downarrow$ ascites $)$ & \\
\hline 10 Choline chloride & Rats fed fat-free and methionine-restricted diet & $0.16 \%$ free & 21 davs & 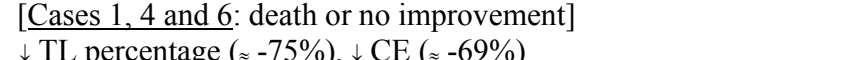 & (Poctet ol 1050) \\
\hline $\begin{array}{ll}11 & \text { (dessicated) } \\
12 & \end{array}$ & & choline & & & (Destcla., 1700) \\
\hline 13 & $\begin{array}{l}\text { Rats fed high-fat }(30 \%) \text { and methionine- } \\
\text { restricted diet }\end{array}$ & $\begin{array}{l}0.32 \% \text { free } \\
\text { choline }\end{array}$ & 21 days & $\downarrow$ TL percentage $(z-73 \%)$ & \\
\hline $\begin{array}{l}14 \text { Choline chloride } \\
15\end{array}$ & $\begin{array}{l}\text { Rats fed steatogen diet ( } 76 \% \text { bolted white corn } \\
\text { meal and 3\% casein) }\end{array}$ & $0.25 \%$ of diet & 65 days & $\downarrow$ fat percentage $(-78 \%)$ & (Shils and Stewart, 1954) \\
\hline 16 Choline chloride & Rats fed $20 \%$ protein choline-deficient diet & $0.26 \%$ of diet & 3 weeks & $\downarrow$ lipid percentage $(-68 \%)$ & (Fritz and Dupont, 1957) \\
\hline 17 Choline & Rats fed high-sucrose $(69 \%)$ and soy protein & $0.3 \%$ of diet & 14 days & $\downarrow$ lipid percentage $(-80 \%)$ & (Olson et al., 1958) \\
\hline $\begin{array}{l}18 \\
19\end{array}$ & $\begin{array}{l}\text { (low methionine) diet } \\
\text { Rats fed high-sucrose }(69 \%) \text { and casein } \\
\text { (adequate methionine) diet } \rightarrow \text { moderate fatty }\end{array}$ & $0.3 \%$ of diet & 14 days & $\downarrow$ lipid percentage $(-51 \%)$ & \\
\hline 21 & $\begin{array}{l}\text { liver } \\
\text { Rats fed high-fat (lard: } 39.9 \% \text { ) and soy protein }\end{array}$ & $0.3 \%$ of diet & 14 days & $\downarrow$ lipid percentage $(-83 \%)$ & \\
\hline 22 & $\begin{array}{l}\text { (low methionine) diet } \\
\text { Rats fed high-fat (lard: } 39.9 \% \text { ) and casein }\end{array}$ & $0.3 \%$ of diet & 14 days & $\downarrow$ lipid percentage $(-75 \%)$ & \\
\hline 24 & $\begin{array}{l}\text { (adequate methionine) diet } \\
\text { Rats fed high-fat (butter fat: } 39.9 \% \text { ) and soy }\end{array}$ & & 14 days & $\downarrow$ lipid percentage $(-71 \%)$ & \\
\hline $\begin{array}{l}25 \\
26\end{array}$ & protein (low methionine) diet & $0.3 \%$ of diet & & & \\
\hline 27 & $\begin{array}{l}\text { Rats fed high-fat (corn oil: } 39.9 \% \text { ) and soy } \\
\text { protein (low methionine) diet }\end{array}$ & $0.3 \%$ of diet & 14 days & $\downarrow$ lipid percentage $(-66 \%)$ & \\
\hline $\begin{array}{l}28 \\
29\end{array}$ & $\begin{array}{l}\text { Rats fed high-fat (butter fat: } 39.9 \% \text { ) and casein } \\
\text { (adequate methionine) diet } \rightarrow \text { less drastic fatty }\end{array}$ & $0.3 \%$ of diet & 14 days & $\downarrow$ lipid percentage $(-70 \%)$ & \\
\hline 30 & $\begin{array}{l}\text { liver } \\
\text { Rats fed high-fat (corn oil: 39.9\%) and casein }\end{array}$ & $0.3 \%$ of diet & 14 days & $\downarrow$ lipid percentage $(-67 \%)$ & \\
\hline 32 & $\begin{array}{l}\text { (adequate methionine) diet } \rightarrow \text { less drastic fatty } \\
\text { liver }\end{array}$ & & & & \\
\hline 34 Choline chloride & $\begin{array}{l}\text { Mice fed high-fat }(28 \%) \text {, low-protein and } \\
\text { hypolipotropic diet }\end{array}$ & $0.002 \%$ of diet & 4 weeks & $\begin{array}{l}\downarrow \text { importantly quantity and size of fat droplets (histological } \\
\text { observations) }\end{array}$ & (Ball, 1964) \\
\hline $\begin{array}{l}35 \\
36 \\
37\end{array}$ & $\begin{array}{l}\text { Rats fed high-sucrose ( }(45.8 \%) \text { and choline- } \\
\text { deficient diet supplemented with histidine, } \\
\text { lysine and threonine }\end{array}$ & $\begin{array}{l}\text { From } 0.01 \text { to } \\
0.64 \% \text { of diet }\end{array}$ & 21 days & $\begin{array}{l}\text { TL percentage (from }-10 \text { to }-84 \% ;-82 \% \text { at } 0.16 \%) \text { : sharp } \\
\text { decrease begins at a level of } 0.06 \% \text { choline } \mathrm{Cl} \text { supplementation } \\
(-60 \%)\end{array}$ & (Young et al., 1965) \\
\hline $\begin{array}{l}38 \text { Choline chloride } \\
39\end{array}$ & $\begin{array}{l}\text { Rats fed basal hypolipotropic and choline- } \\
\text { deficient diet }\end{array}$ & $0.6 \%$ of diet & $-\mathrm{c}$ & $\downarrow$ total esterified FA content $(-89 \%)$ & (Haines and Mookerjea, 1965) \\
\hline 40 & $\begin{array}{l}\text { Rats fed choline-deficient diet for } 10 \text { days then } \\
\text { injected subcutaneously with choline chloride }\end{array}$ & $\begin{array}{l}8,20 \text { or } 40 \mathrm{mg} \\
\text { injected }\end{array}$ & 1 day & $\uparrow$ plasma PL FA level for $40 \mathrm{mg}$ only $(+30 \%)$ & \\
\hline 42 Choline & Rats fed high-fat $(40 \%)$ and $0.1 \%$ niacin diet & $\begin{array}{l}0.30 \text { or } 0.50 \% \text { of } \\
\text { diet }\end{array}$ & 14 days & $\downarrow$ fat percentage (resp. -39 and $-49 \%$ ) compared to $0.15 \%$ choline & (Rikans et al., 1965) \\
\hline $\begin{array}{l}43 \\
44\end{array}$ & & $\begin{array}{l}0.75 \text { or } 1.00 \% \text { of } \\
\text { diet }\end{array}$ & 14 days & $\begin{array}{l}\downarrow \text { fat percentage for } 1 \% \text { choline only }(-36 \%) \text { compared to } 0.50 \% \\
\text { choline }+0.1 \% \text { niacin }\end{array}$ & \\
\hline $\begin{array}{l}45 \\
46\end{array}$ & & $1.00 \%$ of diet & 14 days & $\begin{array}{l}\downarrow \text { total fat }(-1-1 \%) \text {, ) }(-14 \%) \text { and neutral fat }(-22 \%) \text { percentages, }, \\
\text { PL in fat of } 2.2 \% \text { compared to } 0.25 \% \text { choline }+0.1 \% \text { niacin }\end{array}$ & \\
\hline $\begin{array}{l}47 \text { Choline chloride } \\
48\end{array}$ & $\begin{array}{l}\text { Rats fed hypolipotropic and high-sucrose }(62 \%) \\
\text { diet at } 21^{\circ} \mathrm{C}\end{array}$ & $\begin{array}{l}0.2 \% \text { free } \\
\text { choline }\end{array}$ & 21 days & $\downarrow$ lipid percentage $(-66 \pm 12 \%, \mathrm{n}=4$ experiments $)$ & (Chahl and Kratzing, 1966a) \\
\hline 49 Choline & $\begin{array}{l}\text { Rats fed high-sucrose }(69 \%) \text { and casein diet at } \\
21{ }^{\circ} \mathrm{C}\end{array}$ & $\begin{array}{l}0.05,0.1 \text { or } 0.2 \% \\
\text { of diet }\end{array}$ & 21 days & $\downarrow$ lipid percentage (respectively $-70,-74$ or $-75 \%$ ) & (Chahl and Kratzing, 1966b) \\
\hline 51 & $\begin{array}{l}\text { Rats fed high-peanut meal }(30 \%) \text { and casein } \\
\text { diet at } 21^{\circ} \mathrm{C}\end{array}$ & $\begin{array}{l}0.025,0.05,0.1 \\
\text { or } 0.2 \% \text { of diet }\end{array}$ & & $\downarrow$ lipid percentage (respectively $-36,-71,-73$ or $-73 \%$ ) & \\
\hline 52 Choline chloride & Rats fed choline-deficient diet & $0.6 \%$ of diet & 15-18 hours & $\downarrow \mathrm{TG}$ content $(-60 \pm 5 \%, \mathrm{n}=4$ experiments $), \uparrow$ PL $(+21 \%, \mathrm{n}=1)$ & (Lombardi et al., 1968) \\
\hline
\end{tabular}




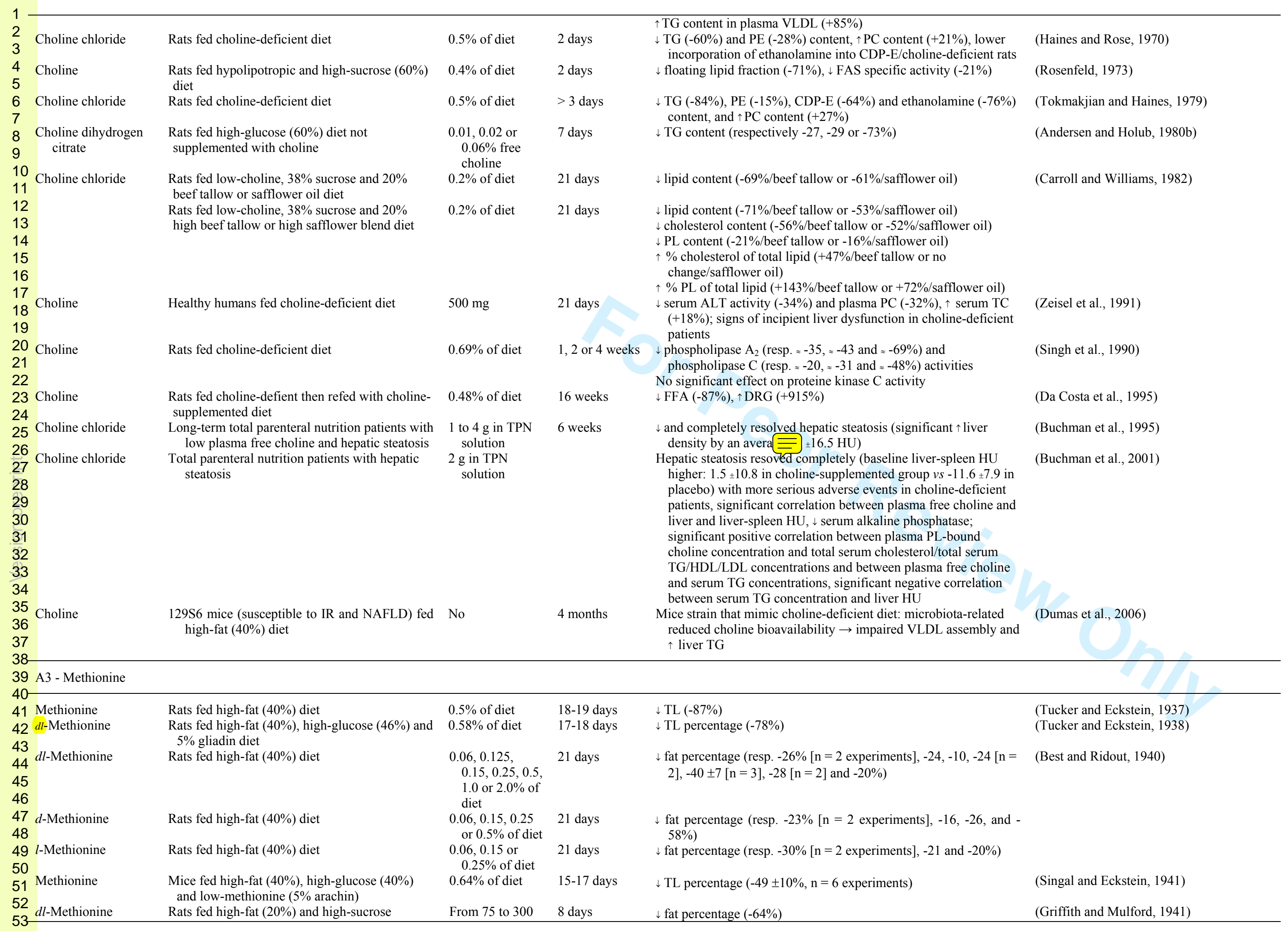




\begin{tabular}{|c|c|c|c|c|c|c|}
\hline & & $(48.9 \%)$ diet & $\mathrm{mg}$ & & & \\
\hline & $\begin{array}{l}\text { Methionine } \\
\text { DL-Methionine }\end{array}$ & $\begin{array}{l}\text { Rats fed high-fat }(40 \%) \text { and } 35 \% \text { gelatin diet } \\
\text { Rats fed high-fat }(35 \%) \text { and low casein }(5 \%)\end{array}$ & $\begin{array}{l}0.774 \% \text { of diet } \\
1.02 \% \text { of diet }\end{array}$ & $\begin{array}{l}21 \text { days } \\
21 \text { days }\end{array}$ & $\begin{array}{l}\downarrow \text { crude FA content }(-69 \%) \\
\downarrow \text { TL percentage }(-63 \%)\end{array}$ & $\begin{array}{l}\text { (Beveridge et al., 1945) } \\
\text { (Ekstein 1952) }\end{array}$ \\
\hline & & diet & & & & \\
\hline & DL-Methionine & $\begin{array}{l}\text { Rats fed choline-deficient, high-sucrose and } \\
20 \% \text { casein diet }\end{array}$ & $\begin{array}{l}0.6 \text { and } 1.0 \% \text { of } \\
\text { diet }\end{array}$ & 14 days & $\downarrow$ fat percentage (resp. -29 and $-33 \%$ ) & (Harper et al., 1954) \\
\hline & DL-Methionine & $\begin{array}{l}\text { Rats fed steatogen diet (76\% bolted white corn } \\
\text { meal and } 3 \% \text { casein) }\end{array}$ & $0.5 \%$ of diet & $\begin{array}{l}27-37 \text { days } \\
65 \text { days }\end{array}$ & $\begin{array}{l}\downarrow \text { lipid percentage (mean decrease of }-65 \% \text { ) } \\
\downarrow \text { fat percentage (mean decrease of }-59 \% \text { ) }\end{array}$ & (Shils and Stewart, 1954) \\
\hline & & & $\begin{array}{l}1.0 \% \text { of diet } \\
\text { From } 0.08 \text { to }\end{array}$ & 65 days & 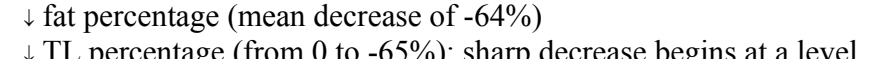 & \\
\hline 0 & Methionine & $\begin{array}{l}\text { Rats fed high-sucrose }(45.8 \%) \text { and methionine- } \\
\text { deficient diet }\end{array}$ & $\begin{array}{l}\text { From } 0.08 \text { to } \\
0.48 \% \text { of diet }\end{array}$ & 21 days & $\begin{array}{l}\downarrow \text { TL percentage (from } 0 \text { to }-65 \%) \text { : sharp decrease begins at a level } \\
\text { of } 0.24 \% \text { methionine supplementation }(-51 \%)\end{array}$ & (Young et al., 1965) \\
\hline 12 & & $\begin{array}{l}\text { Rats fed high-sucrose }(45.8 \%) \text { and methionine- } \\
\text { deficient diet supplemented with } 0.2 \% \text { cystine }\end{array}$ & $\begin{array}{l}\text { From } 0.08 \text { to } \\
0.48 \% \text { of diet }\end{array}$ & 21 days & $\begin{array}{l}\downarrow \text { TL percentage (from } 0 \text { to }-73 \%) \text { : sharp decrease begins at a level } \\
\text { of } 0.16 \% \text { methionine supplementation }(-39 \%)\end{array}$ & \\
\hline $\begin{array}{l}3 \\
4\end{array}$ & L-Methionine & $\begin{array}{l}\text { Rats fed high-sucrose }(69 \%) \text {, casein and } \\
\text { choline-deficient diet at } 21^{\circ} \mathrm{C}\end{array}$ & $\begin{array}{l}0.34 \% \text { of diet } \\
0.68 \% \text { of diet }\end{array}$ & 21 days & $\begin{array}{l}\downarrow \text { lipid percentage }(-67 \%) \\
\downarrow \text { lipid percentage }(-68 \%)\end{array}$ & (Chahl and Kratzing, 1966b) \\
\hline $\begin{array}{l}5 \\
6\end{array}$ & L-Methionine & Rats fed low-protein ( $5 \%$ casein) diet & $\begin{array}{l}0.02,0.2 \text { and } \\
0.5 \% \text { of diet }\end{array}$ & 6 weeks & $\begin{array}{l}\downarrow \text { cholesterol content (respectively } z-17, z-12 \text { and } \mathrm{z}-12 \%, \mathrm{NSS}), \uparrow \\
\operatorname{PL}(+20 \% \text { for } 200 \mathrm{mg} / \mathrm{kg} \text { and no change for other doses) }\end{array}$ & (Osumi et al., 1969) \\
\hline 7 & L-Methionine & $\begin{array}{l}\text { Rats fed low-protein diet (5\% casein) } \\
\text { Rats fed a 9\% casein-based diet }\end{array}$ & $\begin{array}{l}0.5 \% \text { of diet } \\
2.5 \% \text { of diet }\end{array}$ & $\begin{array}{l}3 \text { weeks } \\
3 \text { or } 7 \text { days }\end{array}$ & $\begin{array}{l}\text { } \text { total-coenzyme } \mathrm{A}(+17 \%) \text { and acyl-coenzyme } \mathrm{A}(+6 \%) \text { activities } \\
\mathrm{Affter} 3 \text { days: } \downarrow \text { incorporation of sodium acetate into lipids }(-26 \%)\end{array}$ & (Yokota et al.. 1974) \\
\hline $\begin{array}{l}18 \\
19 \\
20\end{array}$ & Methionine & Mice fed methionine-deficient diet & 2.070 01 पive & $1-15$ days & $\begin{array}{l}\text { After } 7 \text { dass: }: \text { incorporation of sodium acetate into lipids }(+118 \%) \\
\uparrow \text { liver injury but lipid (mainly TG and FFA) accumulation was } \\
\text { less than with choline- and choline+methionine-deficient diets }\end{array}$ & (Caballero et al., 2008) \\
\hline 22 & $\begin{array}{l}\text { A4- Myo-Inositol } \\
\text { (free) }\end{array}$ & & & & 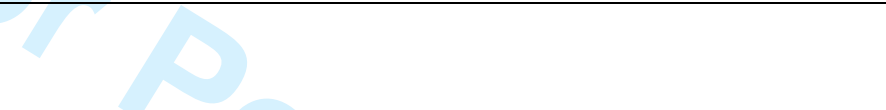 & \\
\hline 25 & Inositol & $\begin{array}{l}\text { Rats injected daily biotin subcutaneously in } \\
\text { conjunction with thiamine, riboflavin, }\end{array}$ & - & - & $\begin{array}{l}\text { Prevents acutely "biotin" type of fatty liver development and } \\
\text { cholesterol accumulation }\end{array}$ & (Gavin and Mchenry, 1941) \\
\hline 27 & Inositol & $\begin{array}{l}\text { pyridoxine and pantothenic acid in the diet } \\
\text { Rats fed high-sucrose }(78 \%) \text { diet }\end{array}$ & $5,10,20$ and 40 & 21 days & $\downarrow$ fat percentage (respectively $-30,-28,-34$ and $-22 \%$ ) & (Engel, 1942) \\
\hline 29 & Inositol & Depancreatized dogs & & - & $\begin{array}{l}\text { Small lipotropic activity but no so marked than a preparation of } \\
\text { lipocaic }\end{array}$ & (Owens, 1942) \\
\hline 31 & Inositol & Rats fed high-fat and cholesterol diet & - & - & Moderate lipotropic action & (Mchenry and Patterson, 1944) \\
\hline 32 & & $\begin{array}{l}\text { Rats fed fat-free diet, thiamine, riboflavin, } \\
\text { pyridoxine and pantothenic acid and/or }\end{array}$ & & & Moderate lipotropic action & \\
\hline 34 & & cholesterol & & & & \\
\hline 35 & Inositol & Rats fed fat-free and methionine-restricted diet & $0.16 \%$ of diet & 21 days & $\downarrow \mathrm{TL}(\approx-34 \%)$ and $\mathrm{CE}(z-45 \%)$ percentages & (Best et al., 1950) \\
\hline 36 & & Rats fed $12 \%$-fat and methionine-restricted diet & $0.32 \%$ of diet & 21 days & No effect on TL percentage & \\
\hline 37 & & $\begin{array}{l}\text { Rats fed high-fat }(30 \%) \text { and methionine- } \\
\text { restricted diet }\end{array}$ & $0.32 \%$ of diet & 21 days & No effect on TL percentage & \\
\hline 39 & Inositol & Humans with hepatic dysfunctions & $\begin{array}{l}1 \mathrm{~g} \text { dissolved in } \\
100 \mathrm{~mL}\end{array}$ & - & $\downarrow$ cholesterolemia & (Gargini, 1951) \\
\hline 40 & Inositol & Rats fed high-fat (51\%) diet & $\begin{array}{l}2.0 \mathrm{mg}(3 \mathrm{x} \\
\text { week })\end{array}$ & 64 days & $\downarrow$ fat content $(-17 \%)$ & (Drill, 1954) \\
\hline 42 & & & $\begin{array}{l}4.0 \mathrm{mg}(3 \mathrm{x} \\
\text { week })\end{array}$ & 64 days & $\downarrow$ fat content $(-24 \%)$ & \\
\hline 44 & Myoinositol & Rats fed high-sucrose ( $84 \%$ ) diet & $30 \mathrm{mg}$ & 7 days & $\begin{array}{l}\downarrow \mathrm{TL} \mathrm{C}(-67 \%) \text { and total cholesterol }(-35 \%) \text { contents, } \downarrow \text { and } \uparrow 11^{-{ }^{4} \mathrm{C}-} \\
\text { acetate incorporation in respectively liver and adipose } \\
\text { cholesterol }\end{array}$ & (Kotaki et al., 1968) \\
\hline 46 & Myo-inositol & Young rats injected large dose of myo-inositol & $40 \mathrm{mg} / \mathrm{rat}$ & 1 hour & $\begin{array}{l}\uparrow \text { PI/PC ratio in liver }(+45 \%) \text { and mitochondrial }(+8 \%) \text { microsomes } \\
\text { after } 1 \text { hour injection }\end{array}$ & (Yagi and Kotaki, 1969) \\
\hline 48 & $\begin{array}{l}3 \text { Inositol } \\
\text { Snositol }\end{array}$ & $\begin{array}{l}\text { Rats fed high--cholesterol ( } 1 \%) \text { diet } \\
\text { Rats fed high-fat }(51 \%) \text { diet }\end{array}$ & $\begin{array}{l}0.5 \% \text { of diet } \\
3 \times 2 \mathrm{mg} \text { per }\end{array}$ & $\begin{array}{l}8 \text { or } 12 \text { weeks } \\
33 \text { days }\end{array}$ & $\begin{array}{l}\downarrow \text { TC content (respectively }-37 \text { and }-56 \%) \\
\downarrow \text { fat percentage }(-17 \%, \mathrm{NS})\end{array}$ & $\begin{array}{l}\text { (Chakrabarti and Baneriee, 1969) } \\
\text { (Laird and Drill, 1971) }\end{array}$ \\
\hline 50 & & & $\begin{array}{l}\text { week } \\
3 \times 4 \mathrm{mg} \text { per } \\
\text { week }\end{array}$ & 33 days & $\downarrow$ fat percentage $(-24 \%)$ & \\
\hline 52 & & Rats fed high-fat $(51 \%)$ diet and administered 3 & $3 \times 2 \mathrm{mg}$ per & 71 days & No significant change & \\
\hline
\end{tabular}


Myo-inositol Rats fed myo-inositol-deficient and balanced diet $\begin{gathered}\text { choline } \\ 0.5 \% \text { of diet }\end{gathered}$

Myo-inositol

Rat dam fed myo-inositol-deficient and lowprotein $(8 \%)$ diet
Rat dam fed myo-inositol-deficient, highfructose $(40 \%)$ and normal-protein $(20 \%)$

Mice (germ-free vs conventional) fed inositoldeficient and high-sucrose $(60 \%)$ diet

Rats fed AIN formula diet supplemented with ats fed high

h-sucrose $(65 \%)$ and

$0.48 \%$ of die

$0.48 \%$ of diet

3 days

12 hours

14 days

23 days

$0.2 \%$ of diet

$0.1 \%$ of diet

13-14 days

16-17 days

FAS $(z-31 \%$ : maximum reached) and ACC/CBX $(z-31 \%)$

specific activity

neutral lipid content (-67\%). maximum reached)

neutral lipid content $(-78 \%)$, no change for PL content

Degree of fatty liver more evident in conventional mice ME activity $/ \mathrm{g}$ protein $(z-50 \%$ in germ-free $v s z-27 \%$ in G6PDH activity conventional micer ACC activity/g protein ( $z-32 \%$ in germ-free $v s$ no change in (ans TG $(+29 \%$

$(+38 \%)$ and total cholestero $\uparrow$ plasma TG $(+42 \%)$, FFA $(+4 \%, N S)$ and total cholesterol $(+6 \%$,

TL (-38\%), cholesterol $(-34 \%)$ and TG $(-66 \%)$ contents

Starch: TL (-2\%, NS), cholesterol (-2\%, NS), and TG (-22\%, NS) (Katayama, 1994) contents; $\uparrow$ PL content $(+9 \%, \mathrm{NS}) ; \downarrow$ G6PDH $(-26 \%, \mathrm{NS})$ and $\mathrm{ME}(-13 \%$, NS) activities

Sucrose: $\downarrow$ TL $(-47 \%)$, cholesterol $(-20 \%)$, and TG $(-74 \%)$ contents; $\uparrow$ PL content $(+6 \%, \mathrm{NS}) ; \downarrow \mathrm{G} 6 \mathrm{PDH}(-43 \%)$ and ME $(-$ 34\%) activities
Starch: $\downarrow$ TL $(-3 \%$, NS) and TG $(-20 \%$, NS) contents; no effect on cholesterol and PL contents; no effect on plasma TG, cholesterol, PL and FFA levels; $\downarrow$ G6PDH (-27\%, NS), ME ($19 \%$, NS), FAS $(-38 \%$, NS), CCE $(-9 \%, \mathrm{NS})$ and CBX $(-9 \%$, Nucrese: activitmg protein

Rats fed high-starch/high-sucrose $(65 \%)$ an

$0.1 \%$ of diet

$12-13$ days (-50\%) and TG $(-81 \%)$ contents; no effect 列 cholesterol, PL and FFA levels; $\downarrow$ G6PDH $(-39 \%$, NS), ME $(-$
$42 \%$, NS), FAS $(-29 \%$, NS), CCE $(-31 \%$, NS) and CBX (-20\%,

NS) activity/mg protein
TL (-34\%), TG $(-80 \%)$, cholesterol $(-13 \%)$ and PL $(-8 \%$, NS)

Rats fed high-sucrose (65\%) diet

$0.515 \%$ of diet

13 days

Rats fed diet with orotic acid $(1.5 \%)$

$1.03 \%$ of diet

8 days

concentrations; $\downarrow$ G6PDH $(-36 \%)$ and ME $(-23 \%)$ activities
$\mathrm{TL}(+5 \%, \mathrm{NS}), \mathrm{TG}(+14 \%, \mathrm{NS})$, cholesterol $(+10 \%$, NS) and $\mathrm{PL}$

$(\approx 0)$ concentrations; $\uparrow \mathrm{G} 6 \mathrm{PD}(+58 \%$, NS) and ME $(+10 \%, \mathrm{NS})$

activity

Rats fed high-starch/high-sucrose $(50.2 \%)$ and $\quad 0.2 \%$ of diet $\quad 14-15$ day

Starch: $\downarrow$ TL $(-19 \%, N S)$, TG $(-41 \%$, NS) and cholesterol $(-5 \%$

NS) levels, $\uparrow$ PL level (+9\%, NS), no change in plasma TG,

NS) and FAS (-4\%, NS

NS) levels $\uparrow$ PL level $(+19 \%)$, no change in plasma TG

cholesterol and PL levels; $\downarrow$ ME $(-19 \%$, NS), G6PDH (-24\%

$0.2 \%+0.07 \%-14-15$ days (

Start: $4 \mathrm{TL}(-34 \%), \mathrm{TG}(-44 \%)$, cholesterol $(-23 \%, \mathrm{NS})$ and PL levels; $\downarrow$ ME $(-23 \%, \mathrm{NS})$, G6PDH $(-41 \%)$ and FAS $(-30 \%, \mathrm{NS})$

activity/mg protein
(Katayama, 1993)

(Beach and Flick, 1982)

(Leclerc and Miller, 1989)

(Ikeda et al., 1992

(Katayama, 1997b)

(Onomi and Katayama, 1997)

(Okazaki and Katayama, 2003) 


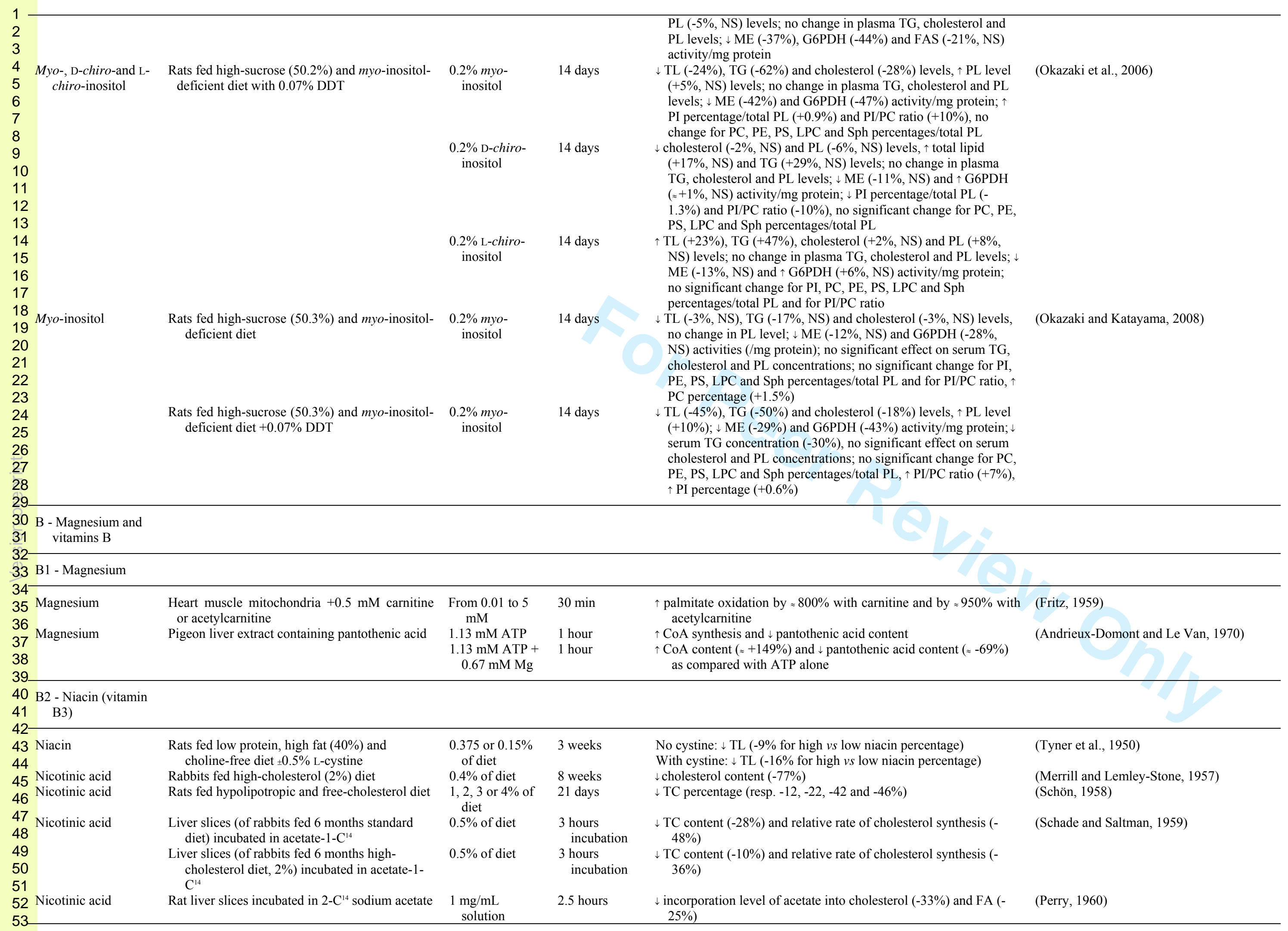




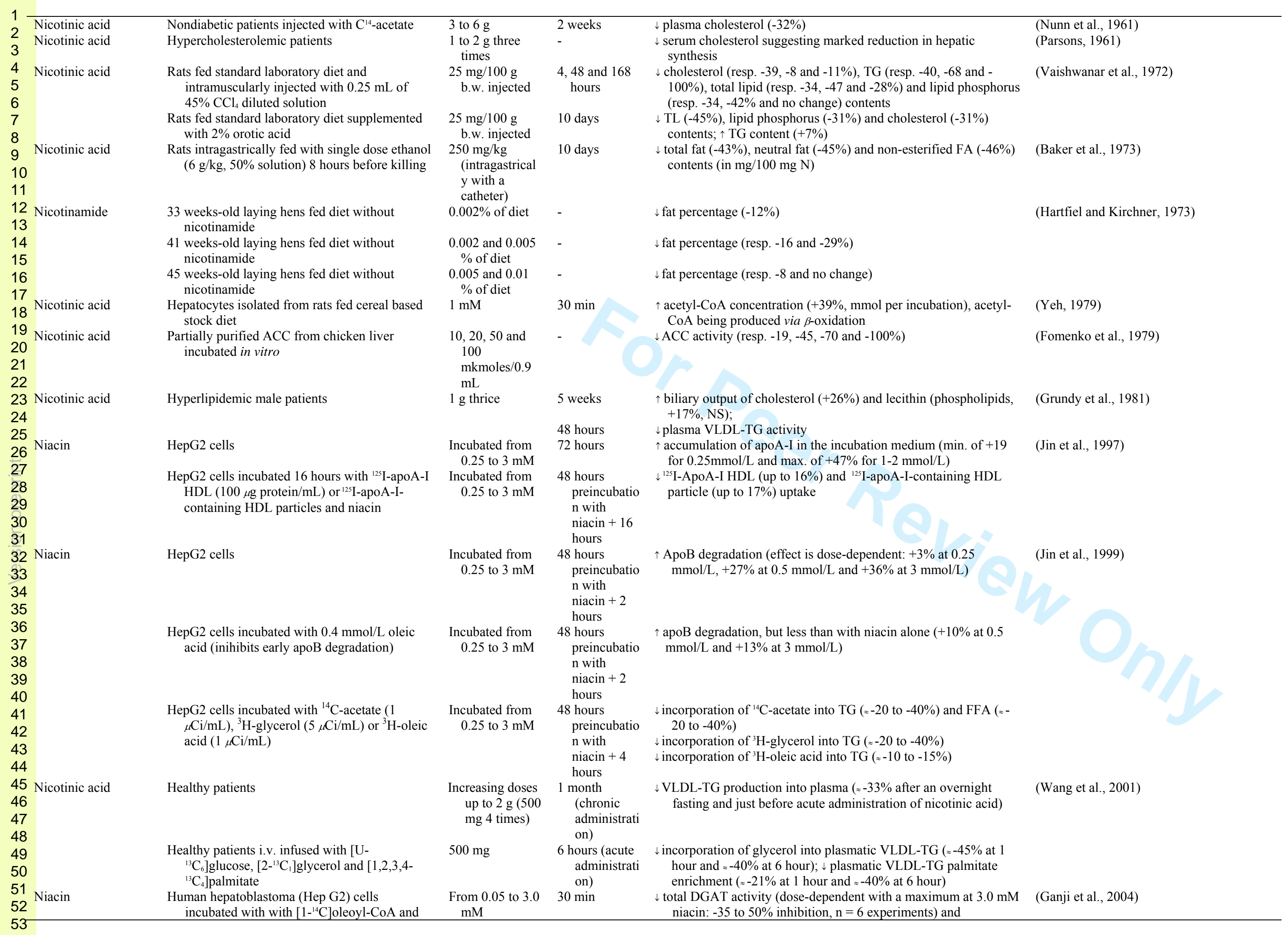




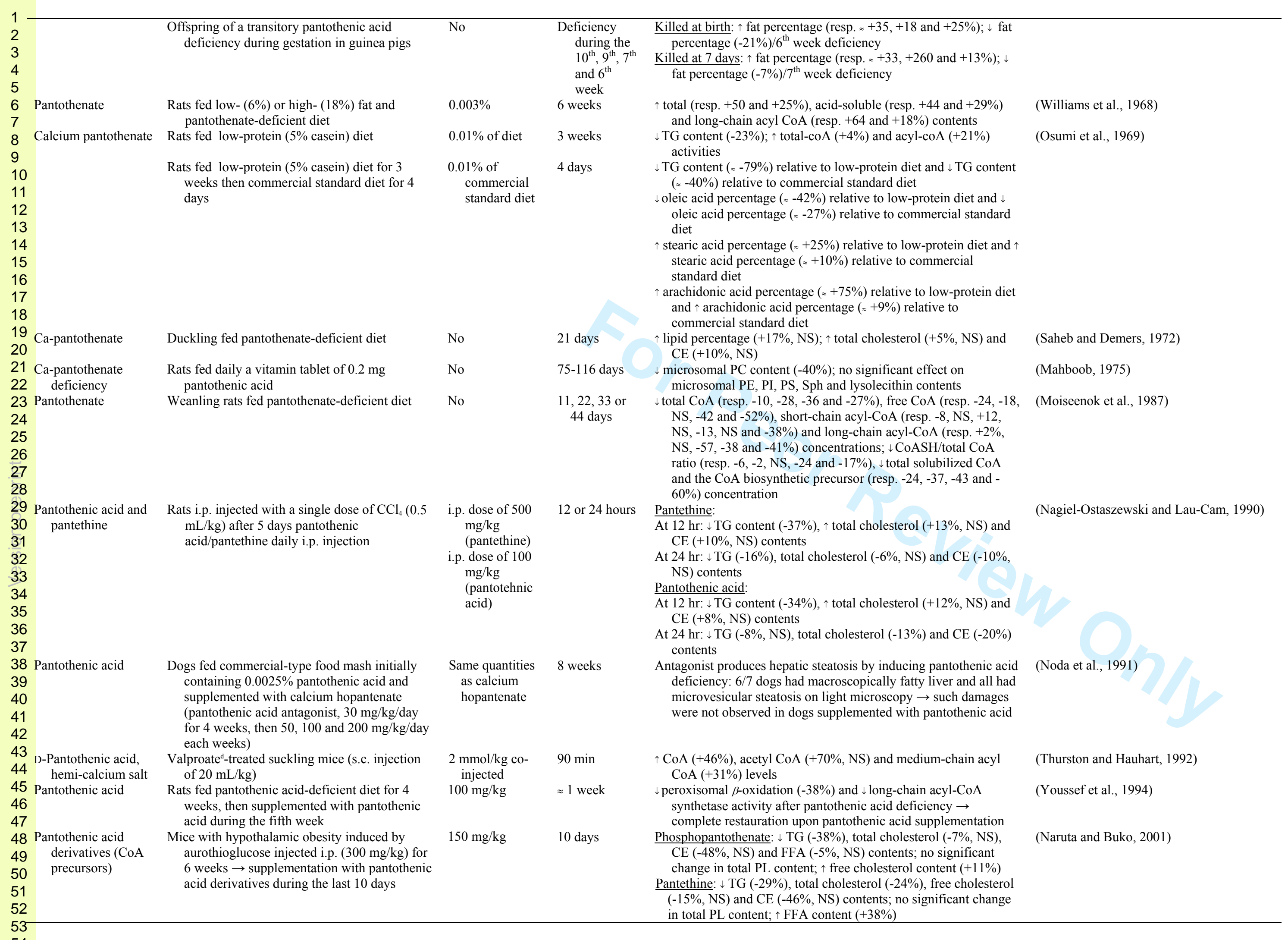




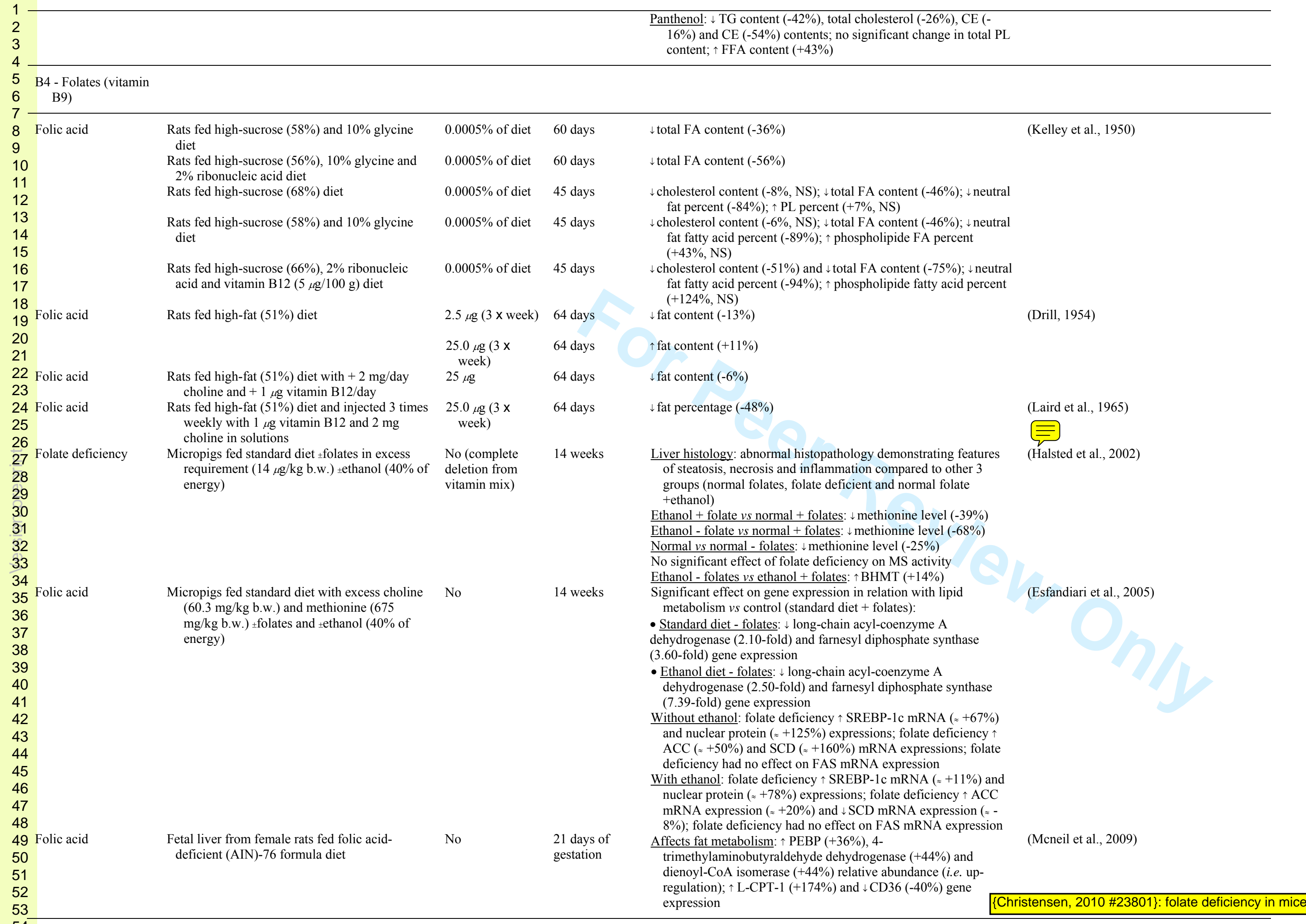




\section{Page 179 of 267}

\section{Critical Reviews in Food Science and Nutrition}

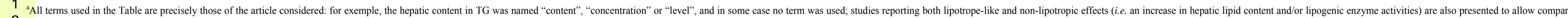

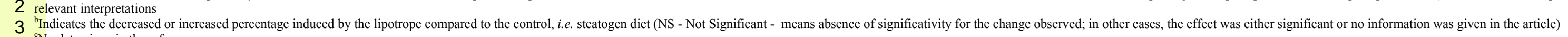

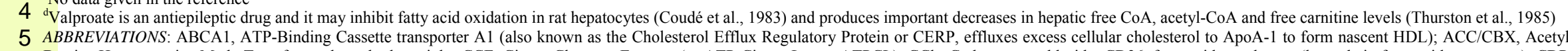

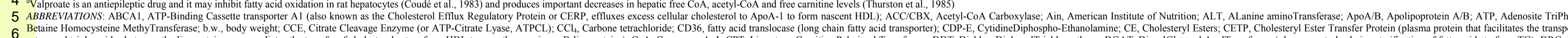

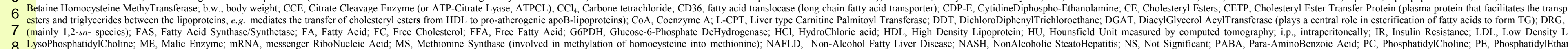

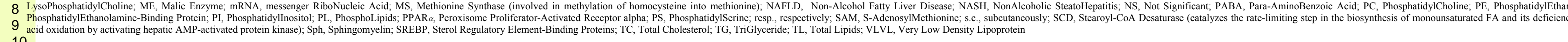

10
11

13

14
15

16

17

19

20

2

24

27 


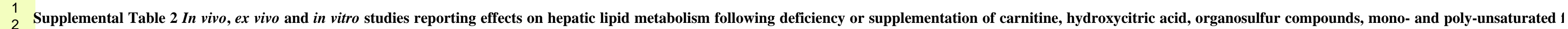

3 short-chain fatty acids, melatonin, tocotrienol, policosanol and para-aminobenzoic acid

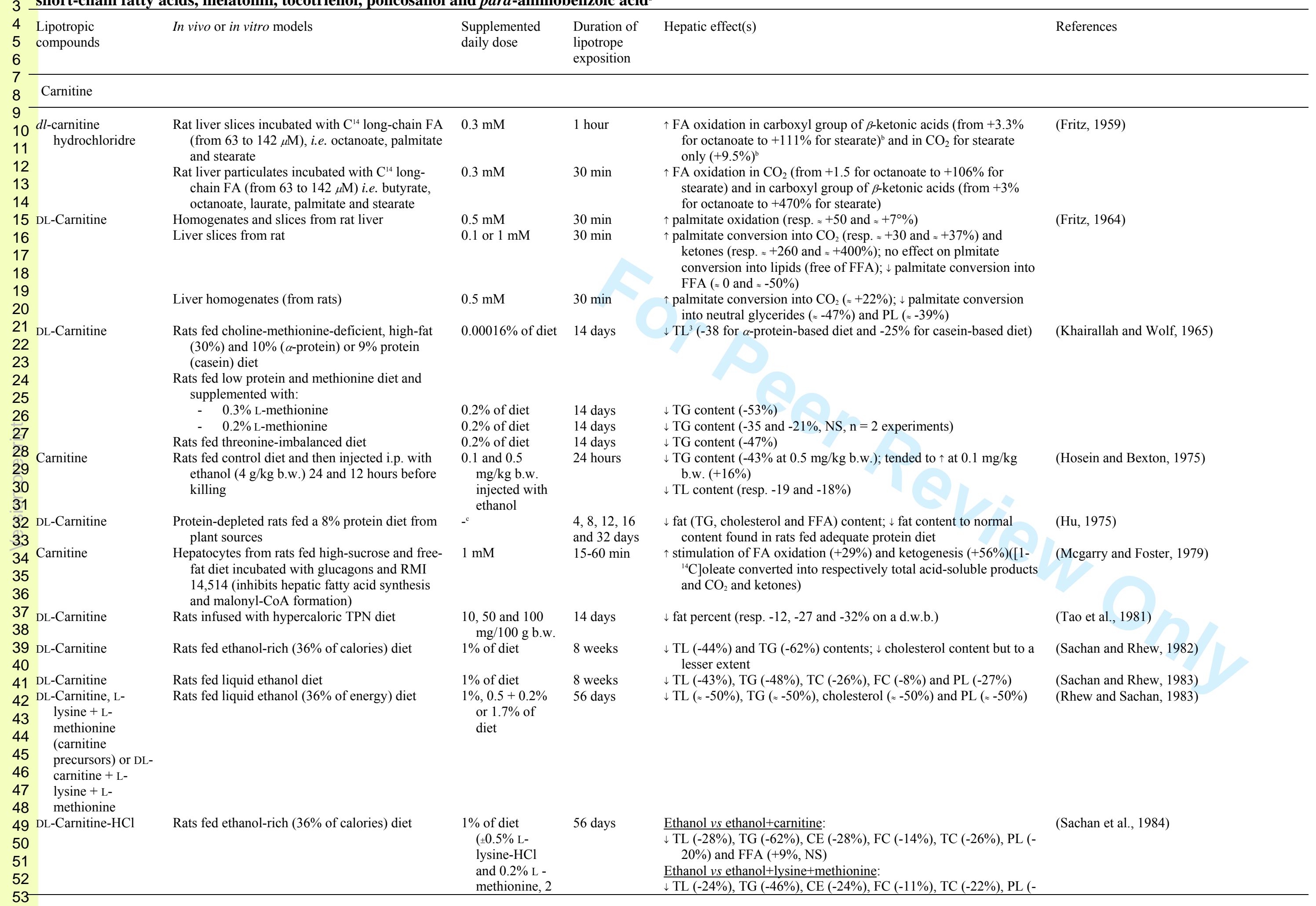




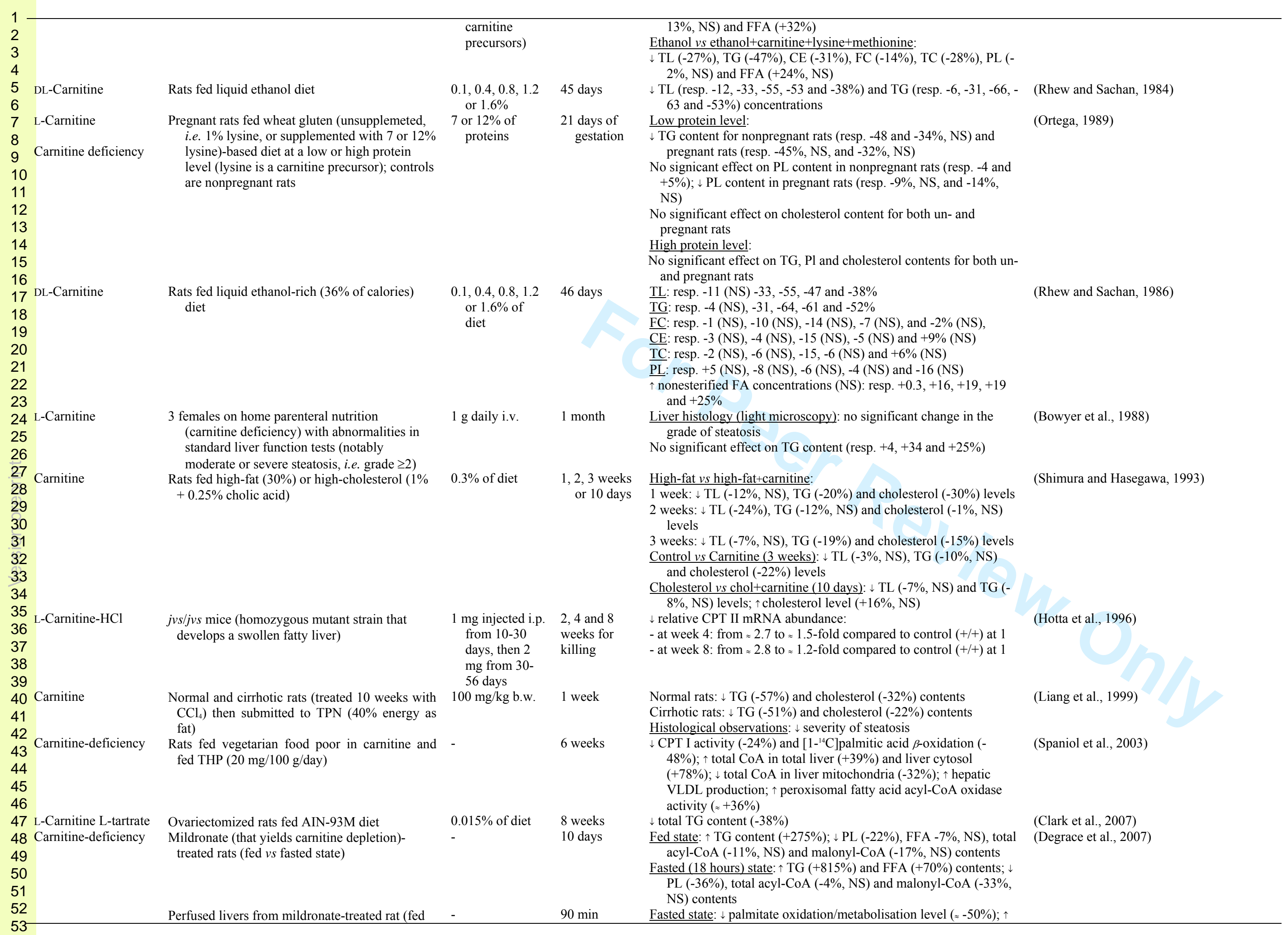




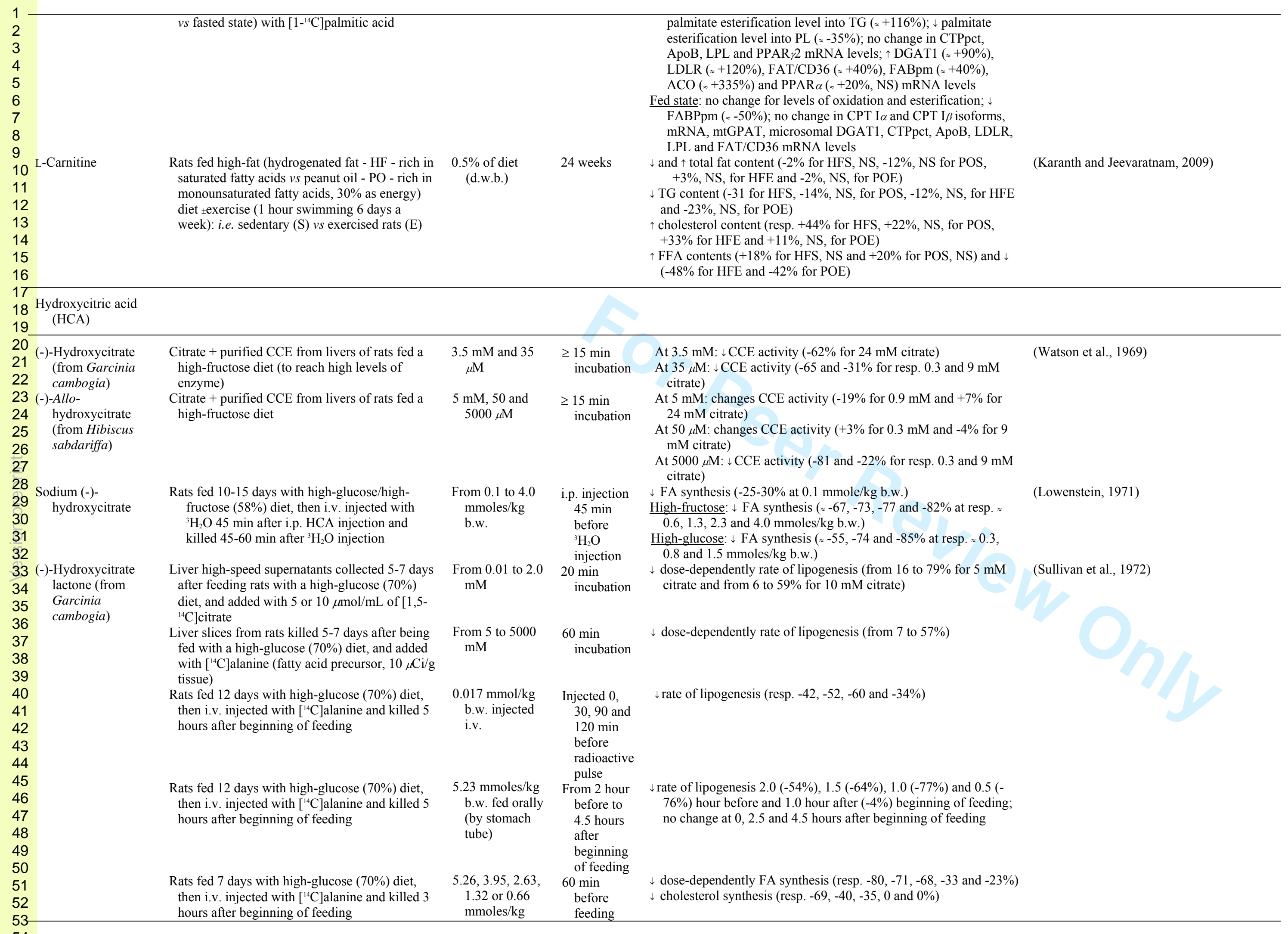




\begin{tabular}{|c|c|c|c|c|c|c|}
\hline $\begin{array}{l}2 \\
3\end{array}$ & & & $\begin{array}{l}\text { b.w. fed orally } \\
\text { (by stomach }\end{array}$ & $\begin{array}{l}\text { (stomach } \\
\text { tube) }\end{array}$ & & \\
\hline 4 & (-)-Hydroxycitrate & Liver high-speed supernatants collected 13 days & $\begin{array}{l}\text { tuae) } \\
1.0 \text { and } 0.1 \mathrm{mM}\end{array}$ & $20 \mathrm{~min}$ & rate of linogenesis (resp -72 and $-52 \%$ at $5 \mathrm{mM}$ citrate -54 and - & \\
\hline 5 & & after feeding rats with a high-glucose $(70 \%)$ & & & $35 \%$ at $10 \mathrm{mM}$ citrate) & \\
\hline $\begin{array}{l}6 \\
7\end{array}$ & (+)-Hydroxycitrate & $\begin{array}{l}\text { diet, and added with } 5 \text { or } 10 \mu \mathrm{mol} / \mathrm{mL} \text { of }[1,5- \\
{ }^{1} \mathrm{C} C \mathrm{Clitrate}\end{array}$ & & & $\uparrow$ rate of lipogenesis (resp. +55 and $+4 \%$ of control at $5 \mathrm{mM}$ citrate; & \\
\hline 8 & & & & & $\begin{array}{l}+31 \% \text { oc control at } 10 \mathrm{mM} \text { citrate); } \downarrow \text { rate of lipogenesis at } 10 \mathrm{mM} \\
\text { citrate }(-10 \%)\end{array}$ & \\
\hline & $\begin{array}{l}\text { (-)-Allo- } \\
\text { Hydroxycitrate }\end{array}$ & & & & $\downarrow$ rate of lipogenesis (resp. -10 and $-6 \%$ at $5 \mathrm{mM}$ citrate; -12 and - & \\
\hline $\begin{array}{l}10 \\
11\end{array}$ & (+)-Allo- & & & & $\begin{array}{l}2 \% \text { at } 10 \mathrm{mM} \text { citrate) } \\
\uparrow \text { rate of lipogenesis (resp. }+31 \text { and }+4 \% \text { at } 5 \mathrm{mM} \text { citrate; }+8 \text { and }\end{array}$ & \\
\hline 12 & $\begin{array}{l}\text { Hydroxyctrtrate } \\
\text { (-)-Hydroxycitrate }\end{array}$ & Rats fed 13 davs with hioh-olucose $(70 \%)$ diet & 263 mmoles $/ \mathrm{kg}$ & $60 \mathrm{~min}$ & $\begin{array}{l}+3 \% \text { at } 10 \mathrm{mM} \text { citrate) } \\
+ \\
\text { trate of filogoenesis (-42\%) }\end{array}$ & \\
\hline $\begin{array}{l}13 \\
14\end{array}$ & $\begin{array}{l}\text { (+)-Hydroxycitrate } \\
(-) \text {-Allo- }\end{array}$ & $\begin{array}{l}\text { then iv. injected with [ }{ }^{14} \text { Clalanine and killed } 3 \\
\text { hours after beginning of feeding }\end{array}$ & $\begin{array}{l}\text { b.w. (by } \\
\text { stomach tube) }\end{array}$ & $\begin{array}{l}\text { before } \\
\text { feeding }\end{array}$ & $\uparrow$ rate of lipogenesis $(+16 \%)$ & \\
\hline 15 & Hydroxycitrate & & & & & \\
\hline $\begin{array}{l}16 \\
17\end{array}$ & $\begin{array}{l}(+)-\text { Allo- } \\
\text { Hydroxycitrate }\end{array}$ & & & & $\uparrow$ rate of lipogenesis $(+4 \%)$ & \\
\hline 18 & (+)-Hydroxycitrate & $\begin{array}{l}\text { Liver from rats fed } 70 \% \text { glucose diet for } 7 \text { days } \\
\text { and killed } 30 \text { min after i.v. iniection of }\end{array}$ & $\begin{array}{c}2.63 \text { mmoless } / \mathrm{kg} \\
\text { bw forally }\end{array}$ & $2,4,6,8$, & $\begin{array}{l}\text { Rate of lipogenesis from }\left[{ }^{[4} \mathrm{C}\right] \text { Calanine: }:-76 \% \text { at } 2 \mathrm{hrs}, \approx-71 \% \text { at } 4 \\
\text { hrse }\end{array}$ & (Sullivan et al., 1974b) \\
\hline $\begin{array}{l}19 \\
20\end{array}$ & & & the last day & 18,21 or & at 12 hrs (NS), $\approx-52 \%$ at 15 hrs (NS),,$+33 \%$ at 18 hrs (NS), $\approx$ & \\
\hline 21 & & & & 24 hours & $\begin{array}{l}+520 \% \text { at } 2 \mathrm{hrs} \text { (NS) and } z+175 \% \text { at } 24 \mathrm{hrs} \\
\text { Rate of lipogenesis from }{ }^{3} \mathrm{H}_{2} 0: z-52 \% \text { at } 2 \text { hrs, } z-61 \% \text { at } 4 \text { hrs, },-\end{array}$ & \\
\hline 22 & & & & & $54 \%$ at 6 hrs, $z-39 \%$ at $8 \mathrm{hrs}, \approx-30 \%$ at $10 \mathrm{hrs}(\mathrm{NS}), \approx 0$ at $12 \mathrm{hrs}$ & \\
\hline $\begin{array}{l}23 \\
24\end{array}$ & & & & & $\begin{array}{l}z+17 \% \text { at } 15 \text { hrs (NS), } z+19 \% \text { at } 18 \text { hrs (NS), } z+63 \% \text { at } 21 \text { hrs } \\
\text { (NS) and } z+60 \% \text { a } 24 \text { hrs (NS) }\end{array}$ & \\
\hline 25 & & Rats fed $70 \%$ glucose diet for 9 days and killed & $2.63,5.26$ or & 4 hours & $\downarrow$ rate of lipogenesis (resp. $-42,-78$ and $-89 \%$ ) & \\
\hline 26 & & 30 min after i.v. injection of [ ["AC]alanine & 10.52 & $\begin{array}{c}\text { before } \\
\text { killina }\end{array}$ & & \\
\hline 27 & & & $\begin{array}{l}\mathrm{mmol} / \mathrm{kg} \text { b.w. } \\
\text { (orally) the }\end{array}$ & & & \\
\hline 29 & & Liver from rats fed $70 \%$ glucose diet for 30 days, & $0.17,0.33,0.66$ & 30 days & Without $10 \mathrm{mM}$ hydroxycitric acid added: $\uparrow$ rate of lipogenesis & \\
\hline 30 & & then incubated in vitro with $10 \mathrm{mM}\left[{ }^{4} \mathrm{C}\right]$ citrate & 1.32 or 2.63 & & (resp. $z+13, \mathrm{NS}, z+25 \%, \mathrm{NS}, z+56, z+104$ and $z+108 \%)$ & \\
\hline 31 & & & $\mathrm{mmol} / \mathrm{kg}$ b.w. & & With $10 \mathrm{mM}$ hydroxycitric acid added: $\uparrow$ rate of lipogenesis (resp. $~$ & \\
\hline 32 & & & (orally) \pm 1 & & $0, z+18 \%, \mathrm{NS}, z+55, z+105$ and $z+118 \%)$ & \\
\hline 33 & & & $\mathrm{mM}$ added in & & Rate of lipogenesis was lower when adding $1 \mathrm{mM}$ hydroxycitric & \\
\hline 34 & & & $\begin{array}{l}\begin{array}{l}\text { vitro after } \\
\text { killing }\end{array} \\
\text {. }\end{array}$ & & acid in vitro (from $\approx-54$ to $\approx-53 \%$ ) & \\
\hline 35 & & Rats fed $70 \%$ glucose diet for 9 days and killed & $0.33,0.66,1.32$ & 11 days & $\downarrow$ rate of lipogenesis from [ ["4 C]alanine (resp. $-27, \mathrm{NS},-21, \mathrm{NS},-76$ & \\
\hline 37 & & $\begin{array}{l}30 \mathrm{~min} \text { after i.v. injection of }\left[{ }^{\left[{ }^{3} \mathrm{C}\right.}\right] \text { ]alanine or } \\
{ }^{\mathrm{H}} \mathrm{O}\end{array}$ & or 2.63 & & and $-43 \%$ ) & \\
\hline 38 & & & $\begin{array}{l}\text { mmol /kg b.w. } \\
\text { (via stomach }\end{array}$ & & $\begin{array}{l}\downarrow \text { rate of lipogenesis from }{ }^{3} \mathrm{H}_{2} 0 \text { (resp. - } 22, \mathrm{NS},-13, \mathrm{NS},-49 \text { and - } \\
37 \% \text { ) }\end{array}$ & \\
\hline 39 & & & & & & \\
\hline 40 & & $\begin{array}{l}\text { Rats fed } 70 \% \text { glucose diet for } 30 \text { days and killed } \\
30 \text { min }\end{array}$ & $\begin{array}{l}0.66,1.32 \text { or } \\
2.63 \mathrm{mmol} / \mathrm{kg}\end{array}$ & 30 days & 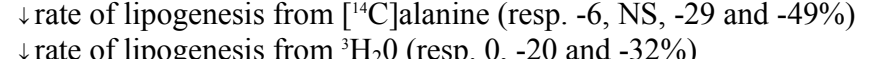 & \\
\hline 41 & & $\begin{array}{l}{ }^{30} \mathrm{H}_{2} 0 \mathrm{~m} \text { after i.v. injection of }\left[{ }^{\circ} \mathrm{C}\right] \text { alanine or } \\
{ }^{2}\end{array}$ & $\begin{array}{l}2.63 \text { mmollkg } \\
\text { b.w. (orally) }\end{array}$ & & & \\
\hline 43 & (-)-Hydroxycitrate & Rats fed $70 \%$ glucose diet & $\begin{array}{l}1.32 \mathrm{mmol} / \mathrm{kg} \\
\text { b.w. }\end{array}$ & 11 days & $\downarrow$ lipid content $(-9 \%, \mathrm{NS})$ & (Sullivan et al., 1974a) \\
\hline $\begin{array}{l}44 \\
45 \\
46\end{array}$ & (-)-Hydroxycitrate & 3-hr meal-fed rats & & 24 hours & $\begin{array}{l}\downarrow \text { significicantly the rate of FA synthesis over } 8 \text {-hr period when } \\
\text { control animals had elevated rates } \\
\downarrow \text { cholesterol synthesis }\end{array}$ & (Sullivan et al., 1974c) \\
\hline 47 & (-)-Hydroxycitrate & Obese Zucker rats fed high-glucose $(70 \%)$ diet & $\begin{array}{l}1.32 \mathrm{mmoles} / \mathrm{kg} \\
\text { twice }\end{array}$ & $7-13$ days & $\begin{array}{l}\text { No significant effect on TL content }(7 \%) \\
\downarrow \mathrm{FA} \text { synthesis rate from }\left[\left[^{4} \mathrm{C}\right] \text { lalanine }(-63 \%) \text { and }{ }^{3} \mathrm{H}_{2} \mathrm{O}(-47 \%)\right.\end{array}$ & (Sullivan et al., 1977) \\
\hline 49 & & Fed and fasted rats fed a $10 \%$-fructose solution & 1.32 mmoles $/ \mathrm{kg}$ & 28 hours & & \\
\hline 50 & & $\begin{array}{l}\text { for } 28 \text { hours } \\
\text { Rotat fed hiva }\end{array}$ & $\begin{array}{l}\text { three times } \\
63 \mathrm{mmolkg}\end{array}$ & 3,6 and 21 . 30 , & 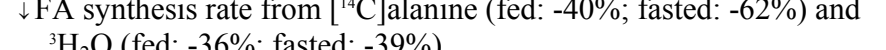 & \\
\hline 51 & & $\begin{array}{l}\text { Ratat fed highh-fructoses }(70 \%) \text { diet for } 6 \text { days and } \\
\text { iv.v. injected with }\left[\left[^{4} \mathrm{C} \text { Clalanine of }{ }^{3} \mathrm{H}_{2} \mathrm{O}\right.\right.\end{array}$ & $\begin{array}{l}2.63 \mathrm{mmmol} / \mathrm{kg} \\
\text { b.w. (oral }\end{array}$ & hours & 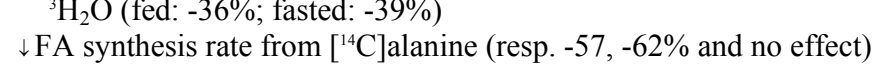 & \\
\hline 52 & & Rats fed high-glucose $(70 \%)$ diet for 6 days and & intubation) & & and ${ }^{3} \mathrm{H}_{2} \mathrm{O}$ (resp. $-59,-31 \%$ and no effect) & \\
\hline
\end{tabular}




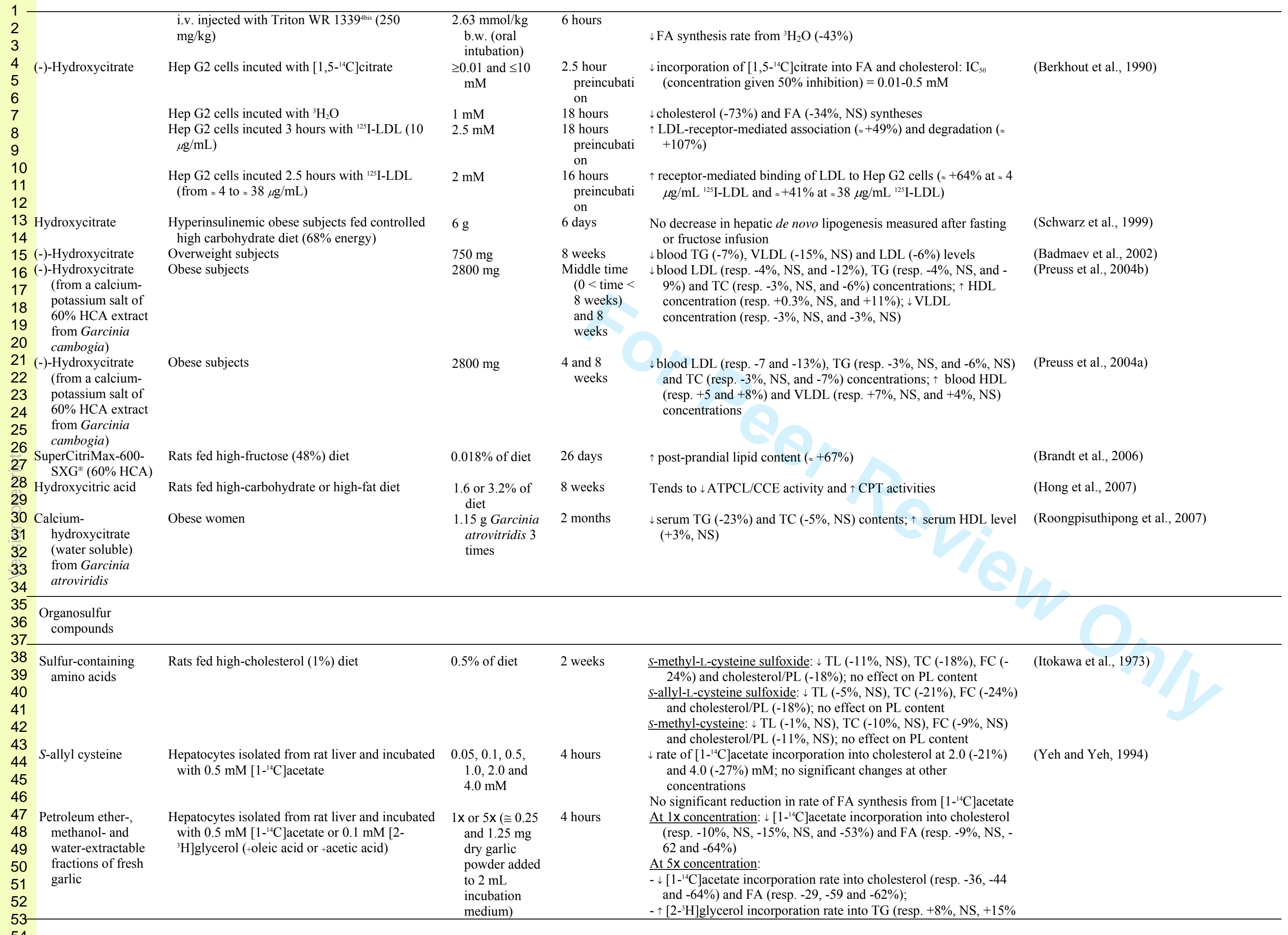




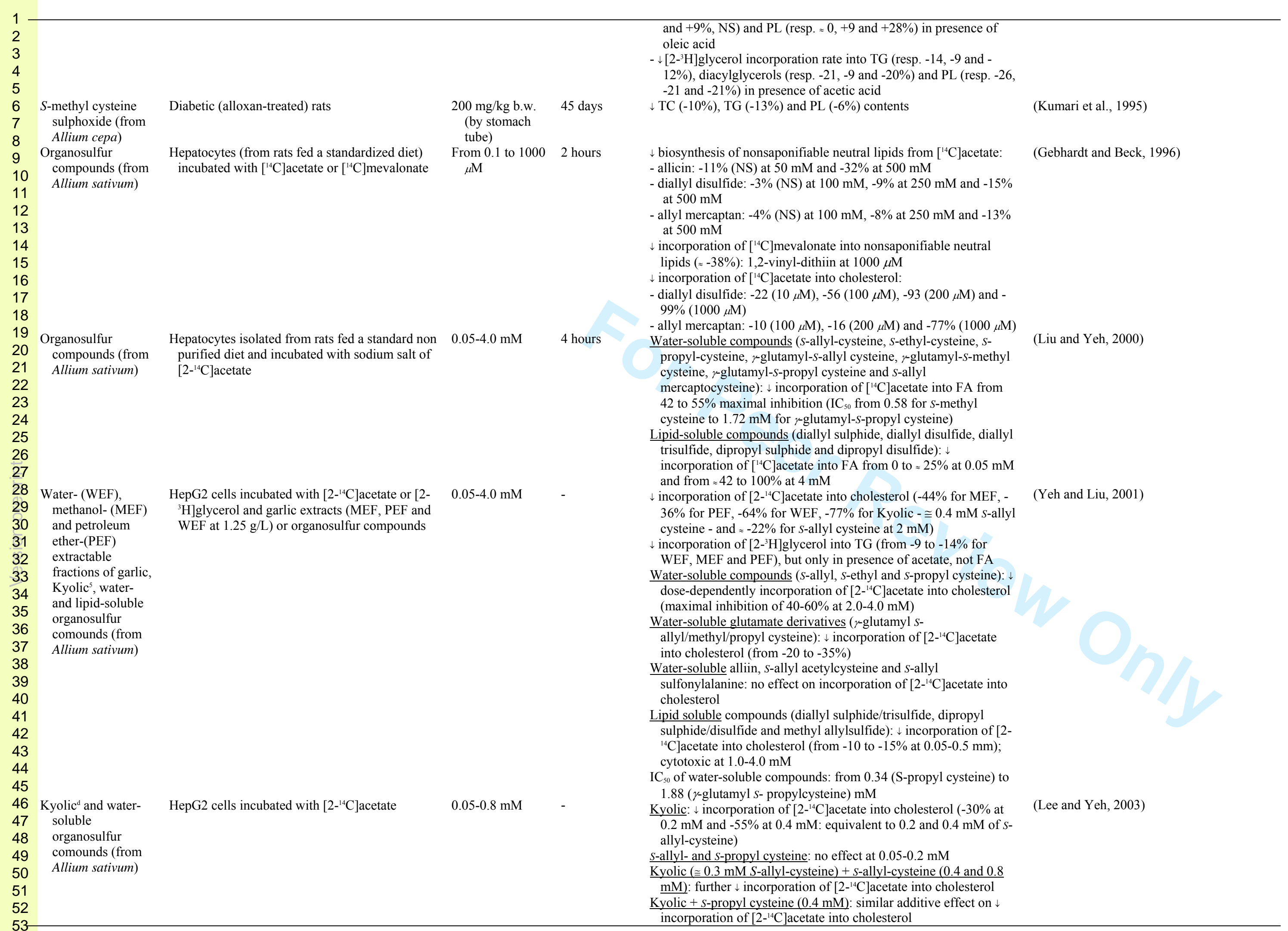




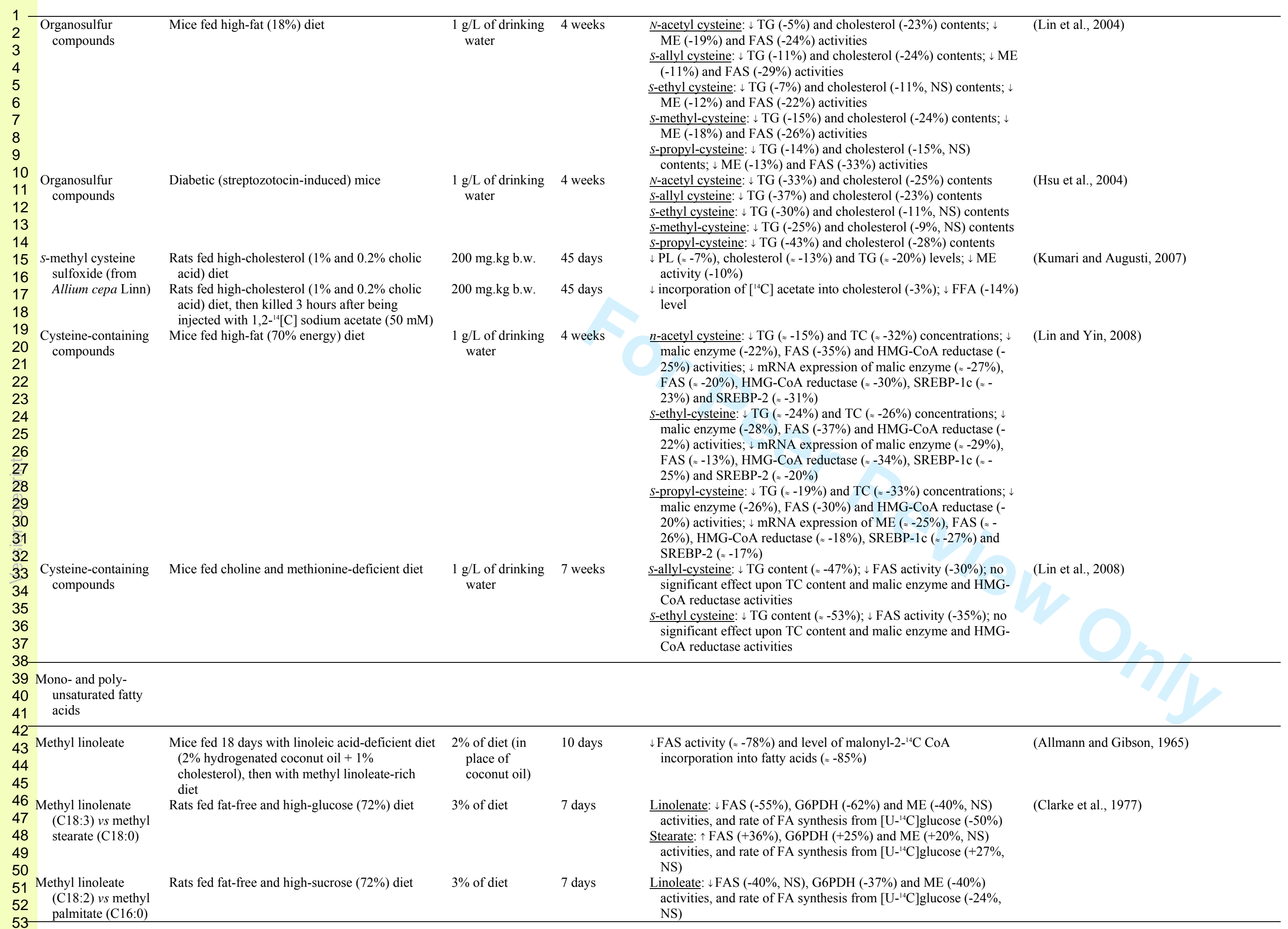




\begin{tabular}{|c|c|c|c|c|c|c|}
\hline 2 & & & & & $\begin{array}{l}\text { Palmitate: } \uparrow \text { G6PDH }(+15 \%, \text { NS }) \text { and ME }(+8 \%, \text { NS }) \text { activities, and } \\
\downarrow \text { FAS activity }(-20 \%, \mathrm{NS}) \text { and rate of FA synthesis from [U- }\end{array}$ & \\
\hline 3 & & & & & 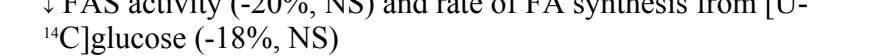 & \\
\hline $\begin{array}{l}4 \\
5\end{array}$ & $\begin{array}{l}\text { Methyl linoleate } \\
\text { (C18:2) vs methyl }\end{array}$ & Rats fed fat-free and high-glucose $(72 \%)$ diet & $3 \%$ of diet & 7 days & 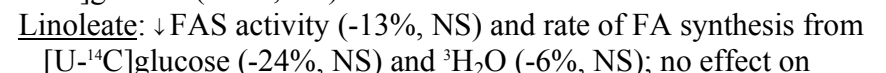 & \\
\hline 6 & oleate $(\mathrm{C} 18: 1)$ & & & & $\mathrm{G} 6 \mathrm{PDH}(0 \%)$ and $\mathrm{ME}(+3 \%, \mathrm{NS})$ activities & \\
\hline 7 & & & & & Oleate: $\downarrow$ FAS $(-38 \%)$, G6PDH $(-39 \%)$ and ME $(-31 \%)$ activities, & \\
\hline & & & & & $\begin{array}{l}\text { and rate of FA synthesis from }\left[\mathrm{U}-{ }^{-1} \mathrm{C}\right] \mathrm{glucose}(-26 \%) \text { and }{ }^{3} \mathrm{H}_{2} \mathrm{O}(- \\
16 \%)\end{array}$ & \\
\hline 10 & Methyl linoleate & Rats fed fat-free and high-glucose $(72 \%)$ diet & Resp. $3 \%$ vs 3\% & 7 days & Linoleate: $\downarrow$ FAS $(-50 \%)$, GPDH $(-64 \%)$ and ME $(-48 \%)$ activities, & \\
\hline 11 & $\begin{array}{l}\text { (C18:2) vs methyl } \\
\text { linolenate (C18:3) }\end{array}$ & & vs $7 \%$ & & 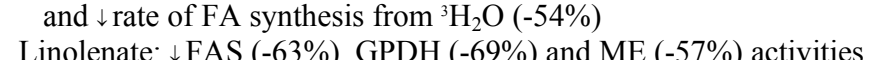 & \\
\hline 12 & vs methyl & & & & 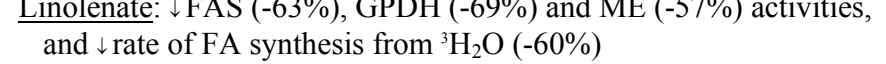 & \\
\hline $\begin{array}{l}13 \\
14\end{array}$ & palmitate (C16:0) & & & & $\begin{array}{l}\text { Palmitate: } \uparrow \text { FAS }(+6 \%, \text { NS), GPDH }(+30 \%) \text { and ME }(+17 \%, N S) \\
\text { activities, and } \uparrow \text { rate of FA synthesis from }{ }^{3} \mathrm{H}_{2} \mathrm{O}(+8 \%, \mathrm{NS})\end{array}$ & \\
\hline 15 & Ethyl linoleate & Rats fed fat-free and high-glucose $(72 \%)$ diet for & $5 \%$ of diet & $1,2,3$ or 4 & $\downarrow$ FA synthesis (resp. $0,-25,41$ and $-59 \%$ ) & (Toussant et al., 1981) \\
\hline $\begin{array}{l}16 \\
17\end{array}$ & $(\mathrm{C} 18: 2)$ & $\begin{array}{l}7 \text { days then supplemented with PUFA, injected } \\
\text { with }{ }^{3} \mathrm{H}_{2} \mathrm{O} \text { and killed } 20 \text { min after injection }\end{array}$ & & days & $\begin{array}{l}\text { ¿FAS (resp. } 0,-19 \%, \text { NS, }-44 \text { and }-56 \% \text { ) and ACC (resp. }-11 \% \text {, NS, } \\
-11 \% \text {, NS, }-39 \text { and }-57 \% \text { ) activities }\end{array}$ & \\
\hline 17 & Arachidonic acid & Rats fed liquid ethanol $(50 \mathrm{~g} / \mathrm{L})$ and fat-free diet & $1 \mathrm{~g} / \mathrm{L}$ & 30 days & $\downarrow$ fat $(-63 \%)$, TG $(-83 \%)$, PL $(-5 \%$, NS $)$ and CE $(-95 \%)$ levels & (Goheen et al.. 1983) \\
\hline $\begin{array}{l}18 \\
19\end{array}$ & Methyl 3-thia-TODT & $\begin{array}{l}\text { Rats fed a conventional pelleted chow diet and } \\
\text { injected palmitic acid (control) }\end{array}$ & $\begin{array}{l}150 \mathrm{mg} / \mathrm{kg} \text { b.w. } \\
\text { (gastric }\end{array}$ & 10 days & $\begin{array}{l}\downarrow \mathrm{TG} \\
\\
\end{array}$ & (Willumsen et al., 1997) \\
\hline $\begin{array}{l}20 \\
21\end{array}$ & & & & & with palmitoyl-L-carnitine as substrate) and peroxisomal $\beta$ - & \\
\hline 22 & & & & & $\begin{array}{l}\text { oxidation } \\
\text { CPT (666\%) } 2 \text { 4-dienovl-CoA reductese }\left(+18^{\circ}\right) \text { ACO }\left(+200^{\circ}\right)\end{array}$ & \\
\hline 23 & & & & & glycerophosphate acyl-transferase $(+137 \%$ in microsomal fraction & \\
\hline 24 & & & & & and $+78 \%$ in mitochondrial fraction), Acyl-CoA:DGAT (+190\%) & \\
\hline 25 & & & & & and CTPpct $(+29 \%)$ activities; $\downarrow$ HMG-CoA reductase $(-80 \%)$ and & \\
\hline 26 & & & & & $\begin{array}{l}\text { Acyl-CoA:CAT }(-33 \% \text { ) activities } \\
\uparrow \text { relative mRNA levels of CP-II ( }+69 \%), 2,4-\text { dienoyl-CoA }\end{array}$ & \\
\hline 28 & & Rat hepatocytes incubated with $\left[11^{-14} \mathrm{C}\right]$ oleic acid & Ratio methyl 3- & 4 hours & $\begin{array}{l}\text { reductase }(+191 \%) \text { and } \mathrm{ACO}(+72 \%) \\
\uparrow \text { FA oxidation }(z+142 \%)\end{array}$ & \\
\hline 29 & & & $\begin{array}{l}\text { thia- } \\
\text { TODT:BSA }=\end{array}$ & & & \\
\hline 31 & & & & & & \\
\hline $\begin{array}{l}32 \\
33\end{array}$ & Triolein & $\begin{array}{l}\text { Transgenic mice fed low carbohydrate }(4.25 \%) \\
\text { and high-protein }(71 \%) \text { diet }\end{array}$ & $10 \%$ of diet & 17 days & SREBP-mediated suppression of FAS promoter & (Moon et al., 2002) \\
\hline 34 & EPA ethyl ester & $\begin{array}{l}\text { Leptin-deficient } o b / o b \text { mice (obesity model) fed } \\
\text { high-carbohydrate and fat-free diet }\end{array}$ & $\begin{array}{l}15 \% \text { triolein }+5 \% \\
\text { EPA or } 20 \%\end{array}$ & 7 days & 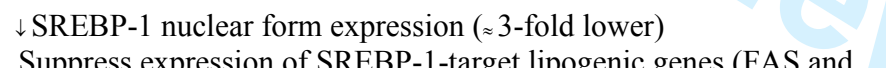 & (Sekiya et al., 2003) \\
\hline 35 & & & tuna fish oil & & $\mathrm{SCD} 1)$ and of $\mathrm{S}_{\mathrm{I}_{4}}$ gene & \\
\hline 37 & & & & & $\begin{array}{l}\text { Induced expression of PPAR } \alpha \text { and ACO } \\
\text { TGG } \text { (resp }-26 \text { and }-440 \text { ) }\end{array}$ & \\
\hline 38 & & & & & NS) contents & \\
\hline 39 & $\begin{array}{l}\text { Omega-3 fatty acids } \\
\text { (from fish oil) }\end{array}$ & $\begin{array}{l}\text { Mice fed high-carbohydrate and fat-free diet for } \\
19 \text { days, then }+ \text { PUFA for } 10 \text { days }\end{array}$ & $2.4 \mathrm{~g} / \mathrm{kg}$ b.w. & 10 days & $\begin{array}{l}\downarrow \text { fat percentage ( }-41 \% \text {, magnetic resonance spectroscopy) and only } \\
\text { slight macrovesicular steatosis (histological observations) }\end{array}$ & (Alwayn et al., 2005a) \\
\hline 41 & & $\begin{array}{l}\text { Leptin-deficient B6.V-Lepob mice fed standard } \\
\text { chow }\end{array}$ & $2.4 \mathrm{~g} / \mathrm{kg}$ b.w. & 30 days & $\begin{array}{l}\text { No difference in fat percentage } \\
\text { naces }\end{array}$ & \\
\hline 42 & $\begin{array}{l}\text { Omega-3 fatty acids } \\
\text { (from fish oil }\end{array}$ & Mice fed fat-free and high-carbohydrate diet & $\begin{array}{l}600 \mu \mathrm{L} \text { (oral or } \\
\text { i.t.) }\end{array}$ & 19 days & $\begin{array}{l}\downarrow \text { macrovevisucular steatosis }(-10 \% \text {, digital mage analysis } \\
\downarrow \text { fat content (resp. }-70 \text { and }-62 \% \text { ) } \\
\text { Had only minor micro-veciculat reatosis }\end{array}$ & (Alwayn et al., 2005b) \\
\hline 44 & $\mathrm{n}-3$ long-chain PUFA & Patients with NAFLD & $1 \mathrm{~g}$ & 12 months & $\begin{array}{l}\text { Had only minor micro-vesicular steatosis } \\
\uparrow \text { Dopler perfusion index (inversely associated with histological }\end{array}$ & (Capanni et al., 2006) \\
\hline 46 & $\begin{array}{l}\text { ethyl esters } \\
\text { (EPA/DHA, }\end{array}$ & & & & $\begin{array}{l}\text { grade of fatty liver, }+62 \% \text { ): } \downarrow \\
0 / 1 \text { degree of steatosis from } \\
0.3 / 35.7 \text { to } 23.8 / 33.3 / 28.6 .4 / 14.3 \text { (percentage of subjects }\end{array}$ & \\
\hline 47 & $0.9 / 1.5)$ & & & & with no or steatosis of various degrees: & \\
\hline 48 & Linseed oil (ALA- & Male and female hamsters fed high-fat diet & & 7 weeks & $\begin{array}{l}\text { absence } / \text { mild/moderate/severe) } \\
\text { Females: } \uparrow \text { PL content }(+3 \%) \text {; no effect on TC, FC, CE and TG }\end{array}$ & (Morise et al., 2006) \\
\hline 50 & rich) & (12.5\% butter $+2.5 \%$ sunflower oil: control) & $\begin{array}{l}\text { (complemente } \\
\text { d w with } 16 \%\end{array}$ & & $\begin{array}{l}\text { contents } \\
\text { Malec } • \text { TC (-25\%) FC (-13\%) CF (-26\%) and TG (-20\%) }\end{array}$ & \\
\hline $\begin{array}{l}51 \\
52\end{array}$ & & & $\begin{array}{l}\text { water }+0.027 \% \\
\text { cholesterol) }\end{array}$ & & contents ; no effect on PL content & \\
\hline
\end{tabular}




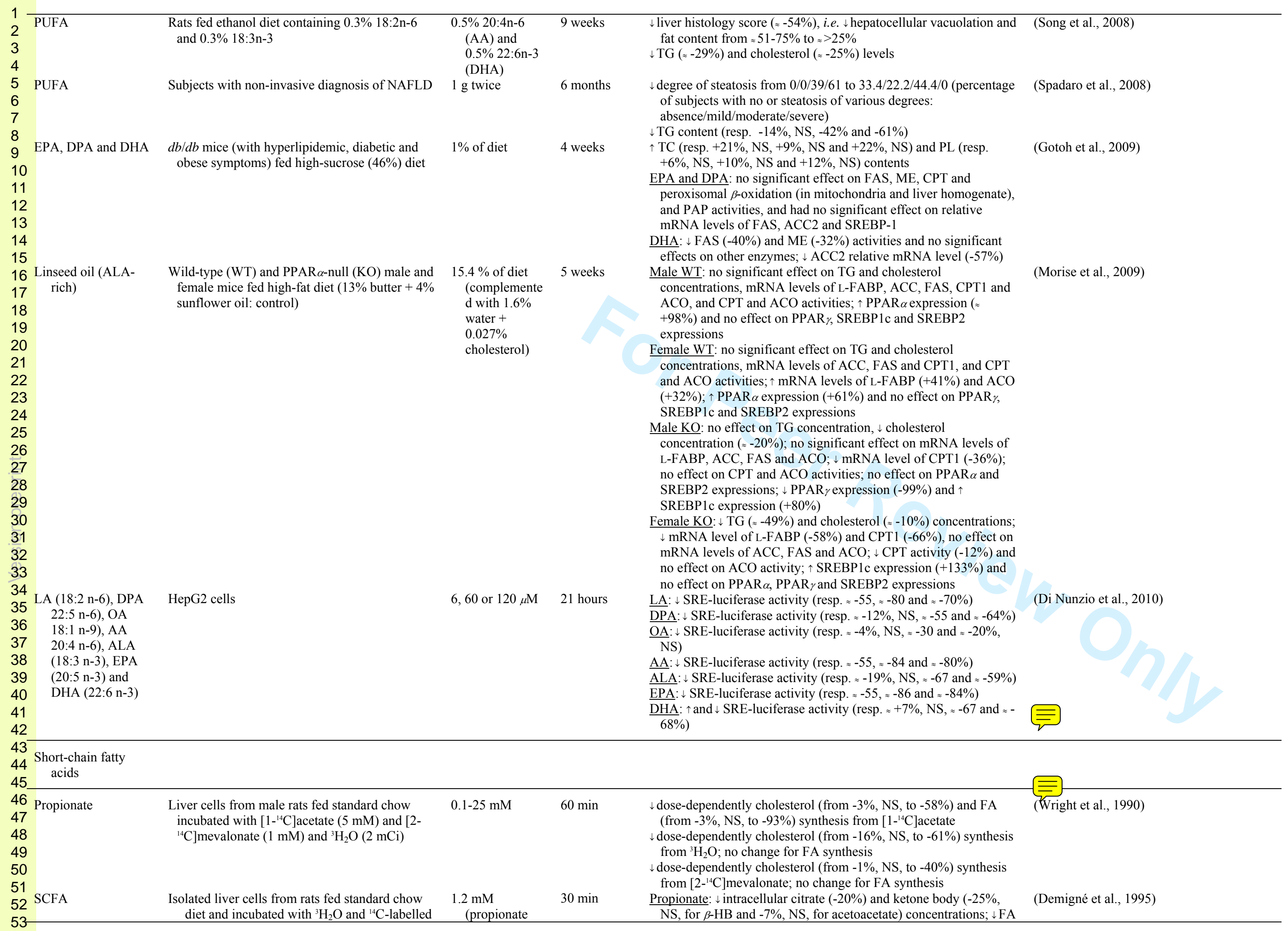




\begin{tabular}{|c|c|c|c|c|c|c|}
\hline$\frac{3}{4}$ & $\begin{array}{l}\text { Lipotropic } \\
\text { compounds }\end{array}$ & In vivo or in vitro models & $\begin{array}{l}\text { Supplemented } \\
\text { daily dose }\end{array}$ & $\begin{array}{l}\text { Duration of } \\
\text { lipotrope } \\
\text { exposition }\end{array}$ & Hepatic effect(s) & References \\
\hline $\mathrm{F}$ & Fibre & & & & & \\
\hline & Pectin (from citrus), & Exp. 1: Rats fed once a day a $10 \%$-fat and $0.2 \%-$ & $5.0 \%$ of diet & 14 days & $\downarrow$ cholesterol (resp. $-14,-5 \%$, NS, and $-3 \%$, NS) $)^{b}$ and long-chain FA & (Kelley and Tsai, 1978) \\
\hline 10 & $\begin{array}{l}\text { gum arabic (Irom } \\
\text { acacia powder) }\end{array}$ & $\begin{array}{l}\text { cholessterol det with } 0 \% \text { cenlluose } \\
\text { Exp. 2: Rats fed ad libitum a } 10 \% \text {-fat and } 0.2 \% \text { - }\end{array}$ & $5.0 \%$ of diet & 23 davs & $\begin{array}{l}\text { (resp. }-20,-11 \%, \mathrm{~N}, \mathrm{Nan}-2 \mathrm{-20}) \text { levels } \\
\downarrow \text { cholesterol (resp }-49,-6 \%, \mathrm{NS} \text {, and }+8 \%, \mathrm{NS} \text { ) and long-chain FA }\end{array}$ & \\
\hline $\begin{array}{l}11 \\
12\end{array}$ & and agar & $\begin{array}{l}\text { cholesterol diet with } 0 \% \text { cellulose for } 14 \text { days } \\
\text { then fed once a day the diet for } 9 \text { days with }\end{array}$ & & & 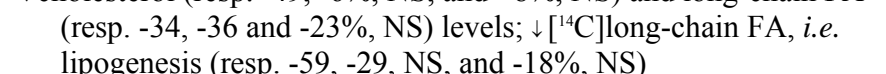 & \\
\hline 13 & & {$\left[{ }^{4} \mathrm{C}\right] g l u c o s e$ in the last meal before killing } & & & & \\
\hline $14 \mathrm{C}$ & Cellulose & Rats fed $10 \%$ fat diet containing adequate & 8 or $16 \%$ of diet & 9 weeks & Marginal zinc content: no signficant effect on cholesterol (resp. - 7 & (Looney and Lei, 1978) \\
\hline 15 & & $\begin{array}{l}\text { amount of dietary copper with either } \\
\text { marginal or abundant }(0.12 \% \text { of diet }) \text { dietary }\end{array}$ & & & $\begin{array}{l}\text { and }-5 \% \text { ) and lipid (resp. }-13 \text { and }-17 \% \text { ) concentration } \\
\text { Abundant zinc content: no signficant effect on cholesterol (resp. }+8\end{array}$ & \\
\hline 17 & & & & & and $+13 \%$ ) and lipid (resp. -16 and $+1 \%$ ) concentration & \\
\hline 18 & $\begin{array}{l}\text { Alfalfa, cellulose or } \\
\text { lignin }\end{array}$ & Rats fed $10 \%$ fat and $1 \%$ cholesterol diet & $5 \%$ of diet & 28 days & $\begin{array}{l}\text { Alfalfa: no significant effect on TC }(-3 \%), \text { FC }(+2 \%) \text { and TG }(- \\
15 \%) \text { contents }\end{array}$ & (Story et al., 1981) \\
\hline 20 & & & & & $\begin{array}{l}\text { Cellulose: no significiant effect on TC }(+15 \%), \mathrm{FC}(+8 \%) \text { and TG } \\
(+15 \%) \text { contents }\end{array}$ & \\
\hline $\begin{array}{l}21 \\
22\end{array}$ & & & & & $\begin{array}{l}\frac{\text { Lignin no significant effect on TC }(-19 \%) \text { and FC }(-1 \%) \text { contents; }}{\downarrow \mathrm{TG} \text { content }(-85 \%)} \\
\text { a }\end{array}$ & \\
\hline 23 & $\begin{array}{l}\text { Cellulose, lignin or } \\
\text { pectin }\end{array}$ & Rats fed $10 \%$ fat and $0.5 \%$ cholesterol diet & $5 \%$ of diet & 28 days & 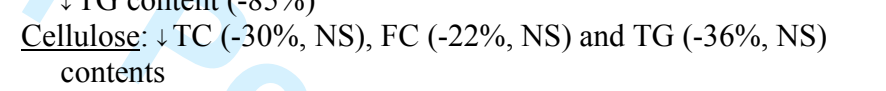 & \\
\hline 25 & & & & & $\begin{array}{l}\text { Lignin: } \\
\text { Pectin: }(-66 \%), \text { FC }(-18 \%, \text { NS }) \text { and TG }(-18 \% \text {, NS) contents } \\
\text { PTC }(-75 \%) \text {, FC }(-27 \%, \text { NS }) \text { and TG }(-58 \%) \text { contents }\end{array}$ & \\
\hline 26 & $\begin{array}{l}\text { Neutral detergent } \\
\text { fiber (from }\end{array}$ & $\begin{array}{l}\text { Rats fed a } 11 \% \text {-fat and fibre-free diet: } \\
\text { - liver slices incubated with [U-"AC]glucose } 10\end{array}$ & $30 \%$ of diet & 1 month & $\begin{array}{l}\text { cholesterol concentration (- }-9 \%) \text {; H HMG-CoA reductase (i.e. } \\
\text { HMG-CoA/mevalonate ratio by } 36 \% \text { ) }\end{array}$ & (Thomas et al., 1983) \\
\hline 28 & blackgram) & $\begin{array}{l}\mathrm{mM}(5 \mu \mathrm{C} \text { i) } \\
\text { - liver slices from rats injected i.p. } 3 \text { hours }\end{array}$ & & & $\uparrow$ incorporation of $\left[\mathrm{U}-{ }^{14} \mathrm{C}\right] \mathrm{glu}$ lucose into cholesterol $(+80 \%)$ & \\
\hline 30 & & before killing with $1 \mathrm{~mL}$ of $\left[1,22^{-{ }^{-1} \mathrm{C}} \mathrm{C} \mathrm{Na}-\right.$ & & & $\uparrow$ incorporation of $\left[1,2-{ }^{-1} \mathrm{C}\right] \mathrm{Na}$-acetate into cholesterol $(+258 \%)$ & \\
\hline 32 & Citrus pectin & Rats fed standard diet containing $14 \%$ cellulose & $10 \%$ of diet & 5 weeks & $\downarrow \mathrm{TL}(-68 \%)$ and TC $(-63 \%)$ contents & (Rotenberg and Eggum, 1986) \\
\hline 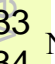 & $\begin{array}{l}\text { (purified) } \\
\text { Neutral detergent }\end{array}$ & Rats fed diets with various contents in & $0-14 \%$ of diet & 28 days & From 2.83 to $11.17 \%$ fiber, i.e. $+8.34 \%$ of fiber & (Stewart et al., 1987) \\
\hline 35 & $\begin{array}{l}\text { fiber (from wheat } \\
\text { bran) }\end{array}$ & $\begin{array}{l}\text { carbohydrate (C: 40-60\%), lipid (L: 9-19\%) } \\
\text { and protein (P: } 9-37 \% \text { ), i.e. } n=32 \text { diets }\end{array}$ & & & $-\frac{44 \% \mathrm{C}, 11 \% \mathrm{~L} \text { and from } 37.01 \text { to } 27.31 \% \mathrm{P}: \downarrow \text { cholesterol }(-14 \%)}{\text { and TG }(-24 \%) \text { contents }}$ & \\
\hline $\begin{array}{l}36 \\
37\end{array}$ & & & & & - from 44 to $56 \% \mathrm{C}$, from 17 to $11 \% \mathrm{~L}$ and from 15.31 to $31.01 \% \mathrm{P}$ : & \\
\hline $\begin{array}{l}38 \\
39\end{array}$ & & & & & $-\frac{56 \% \mathrm{C}, 17 \% \mathrm{~L} \text { and from } 19.01 \text { to } 9.31 \% \mathrm{P}: \downarrow \text { cholesterol content }(-}{6 \%) \text { and } \uparrow \mathrm{TG} \text { content }(+47 \%)}$ & \\
\hline $40^{1}$ & $\begin{array}{l}\text { Methylcellulose (low, } \\
\text { medium and high } \\
\text { viscosity: LV MV }\end{array}$ & Rats fed sucrose-based diet & $8 \%$ of diet & 10 days & $\begin{array}{l}\text { MV and HV: } \downarrow \text { rate of FA synthesis compared to LV (resp. - }-22 \% \text {, } \\
\mathrm{NS} \text {, and }-55 \% \text {, NS); } \downarrow \text { T TG concentration (resp. - } 14 \% \text {, NS, and - } \\
11 \% \text {, NS) compared to LV; no effect on rate of cholesterol }\end{array}$ & (Topping et al., 1988) \\
\hline 42 & and HV) & & & & synthesis and on cholesterol concentration & \\
\hline $43 \mathrm{I}$ & $\begin{array}{l}\text { Particulate (alfalfa, } \\
\text { cellulose or wheat }\end{array}$ & Rats fed a $14 \%$-fat diet & 5 (pectin and & 28 days & $\uparrow$ cholesterol content (resp. $+20,+16,0,+20,+14$ and $+23 \%)$ & (Kritchevsky et al., 1988) \\
\hline 45 & $\begin{array}{l}\text { cellulose or wheat } \\
\text { bran), }\end{array}$ & & & & $\begin{array}{l}\uparrow \text { and } \downarrow \text { T content }(\text { resp. }+2,2,-5,+8,-2,-32 \text { and }-26 \%) \\
\downarrow \text { PL content (resp. } 0,-27,-5,0,0 \text { and }-11 \% \text { ) }\end{array}$ & \\
\hline 46 & soluble/ionic & & (particulate & & $\uparrow P C$ content (resp. $+25,+8,+10,+24,+13$ and $+11 \%)$ & \\
\hline 47 & (pectin) and & & fiber and & & $\downarrow \mathrm{PE}$ (resp. $-11,0,-10,-14,-11$ and $-6 \%$ ) and Sph (resp. $-53,-23,-$ & \\
\hline 48 & soluble/noionic & & Metamucil) of & & $20,-39,-25$ and $-26 \%)$ contents & \\
\hline & $\begin{array}{l}\text { fiber (guar gum or } \\
\text { Metamucile) }\end{array}$ & & & & $\uparrow$ and $\downarrow$ LPC (resp. $-8,+3,+7,-15,+8$ and $+15 \%$ ) and PI+PS (resp. & \\
\hline 50 & $\begin{array}{l}\left.\text { Metamucil }{ }^{*}\right) \\
\text { Citrus pectin }\end{array}$ & Rats fed fiber-free diet & $\begin{array}{l}1,3,6 \text { or } 10 \% \text { of } \\
\text { diet }\end{array}$ & 26 days & $\begin{array}{l}\downarrow \text { cholesterol (resp. } \approx-7 \%, \mathrm{NS}, \approx--9 \%, \mathrm{NS}, \approx-11 \%, \mathrm{NS}, \text { and } \approx-13 \% \\
\text { NS) and TG (resp. } \approx-23 \%, \mathrm{NS}, \approx-41, \approx-59 \text { and } \approx-73 \% \text { ) } \\
\text { concentrations }\end{array}$ & (Ide and Horii, 1989) \\
\hline $53+$ & Wheat bran (GMD: & Rats fed high-sucrose $(49 \%)$ diet containing 5\% & $5,7.5$ or $10 \%$ of & 6 weeks & Fine beet fiber: $\downarrow \mathrm{TG}$ (resp. $-20,-34$ and $-37 \%$ ) and cholesterol & (Klopfenstein, 1990) \\
\hline
\end{tabular}




\begin{tabular}{|c|c|c|c|c|c|c|}
\hline 5 & $\begin{array}{l}492 \mu \mathrm{m}) \text {, or coarse } \\
(436 \mathrm{\mu m} \text { ) and fine } \\
(185 \mathrm{~m}) \text { sugar } \\
\text { beet fiber }\end{array}$ & cellulose (GMD: $179 \mu \mathrm{m}$ ) & $750 \%$ of diet & & 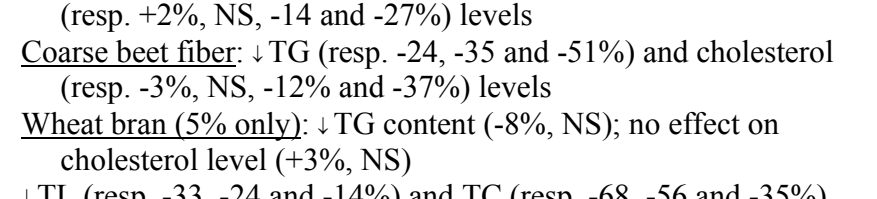 & \\
\hline & $\begin{array}{l}\text { Oat bran, pectin or } \\
\text { psyllium }\end{array}$ & $\begin{array}{l}\text { Rats fed } 10 \% \text {-fat and } 0.3 \% \text {-cholesterol diet } \\
\text { containing } 7.5 \% \text { cellulose }\end{array}$ & $7.5 \%$ of diet & 3 weeks & $\begin{array}{l}\downarrow \text { TL (resp. - } 33,-24 \text { and }-14 \% \text { ) and TC (resp. }-68,-56 \text { and }-35 \% \text { ) } \\
\quad \text { levels }\end{array}$ & (Arjmandi et al., 1992a) \\
\hline $\begin{array}{l}8 \\
9\end{array}$ & $\begin{array}{l}\text { Pectin, psyllium or } \\
\text { oat bran }\end{array}$ & $\begin{array}{l}\text { Rats fed 10\%-fat and } 0.3 \% \text {-cholesterol diet } \\
\text { containing } 10 \% \text { cellulose }\end{array}$ & $10 \%$ of diet & 3 weeks & $\begin{array}{l}\text { Pectin and psyllium: } \\
\text { and }-40 \% \text { ) levels (resp. }-29 \text { and }-29 \% \text { ) and TC (resp. }-54\end{array}$ & (Arjmandi et al., 1992b) \\
\hline $\begin{array}{l}10 \\
11 \\
12 \\
13 \\
14 \\
15\end{array}$ & Guar gum & $\begin{array}{l}\text { Quails fed } 5 \% \text { cellulose diet } 550 \mathrm{ppm} \text { of } \\
\text { tocotrienol-rich fraction (from palm oil) }\end{array}$ & $5 \%$ of diet & 4 or 44 weeks & 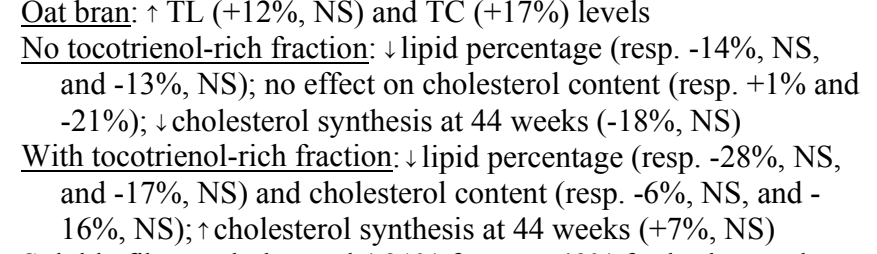 & (Hood and Sidhu, 1992) \\
\hline $\begin{array}{l}16 \\
17 \\
18 \\
19 \\
20\end{array}$ & $\begin{array}{l}\text { Fiber from defatted } \\
\text { oat, barley or } \\
\text { wheat }\end{array}$ & $\begin{array}{l}\text { Rats fed hypercholesterolemic ( } 1 \% \text { cholesterol } \\
\text { and } 0.25 \% \text { sodium cholate) diet containing } \\
5 \% \text { cellulose }\end{array}$ & $\begin{array}{l}\text { Resp. } 1.9,2.8 \\
\text { and } 0.6 \\
\text { soluble fiber } \\
\text { or } 3.1,2.2 \text { and } \\
4.4 \% \\
\text { insoluble fiber }\end{array}$ & 9 days & $\begin{array}{l}\frac{\text { Soluble fiber: }}{11} \downarrow \text { cholestererol }(-31 \% \text { for oat, }-49 \% \text { for barley, and - } \\
\text { Insoluble wheat, NSS) concentration } \\
\text { and }-8 \% \text { for whoeat, NS) concentration }\end{array}$ & (Oda et al., 1993) \\
\hline $\begin{array}{l}21 \\
22 \\
23\end{array}$ & $\begin{array}{l}\text { Wheat bran, psyllium } \\
\text { husk or oat bran }\end{array}$ & $\begin{array}{l}\text { Rats fed basal diet containing } 9.09 \% \text { wheat bran, } \\
4.00 \% \text { psyllium husk or } 15.38 \% \text { oat bran }\end{array}$ & $7.2-7.6 \%$ of fiber & $\begin{array}{r}3.5,10,15 \text { and } \\
18.5 \text { months }\end{array}$ & $\begin{array}{l}\text { Cholesterol: no significant changes (except a tendency to } \downarrow \text { at } 15 \\
\text { and } 18.5 \text { months for wheat bran and psyllium) } \\
\text { CE: no significant changes (except a slight tendency to } \downarrow \text { at } 18.5\end{array}$ & (Schneeman and Richter, 1993) \\
\hline $\begin{array}{l}24 \\
25 \\
26\end{array}$ & & & & & $\begin{array}{l}\text { TG: no significant changes (except a slight tendency to } \downarrow \text { at } 18.5 \\
\text { months) }\end{array}$ & $\equiv$ \\
\hline 28 & $\begin{array}{l}\text { Oat bran, guar gum, } \\
\text { cellulose or xylan }\end{array}$ & $\begin{array}{l}\text { Hamsters fed hypercholesterolemic }(0.1 \% \\
\text { cholesterol and } 10 \% \text { fat) diet }\end{array}$ & $\approx 10 \%$ of diet & 4 weeks & $\begin{array}{l}\downarrow \text { cholesterol }(-18 \% \text { for oat bran, NS, }-24 \% \text { for guar gum, NS, and - } \\
29 \% \text { for xylan, NS) concentration; } \uparrow \text { cholesterol }(+44 \% \text { for } \\
\text { cellulose) concentration }\end{array}$ & (Jonnalagadda et al., 1993) \\
\hline $\begin{array}{l}29 \\
30 \\
31 \\
32 \\
33\end{array}$ & Prune fiber or pectin & $\begin{array}{l}\text { Rats fed high cholesterol }(1 \%+0.1 \% \text { cholic } \\
\text { acid) AIN-76 diet }\end{array}$ & 3 or $6 \%$ of diet & 28 days & 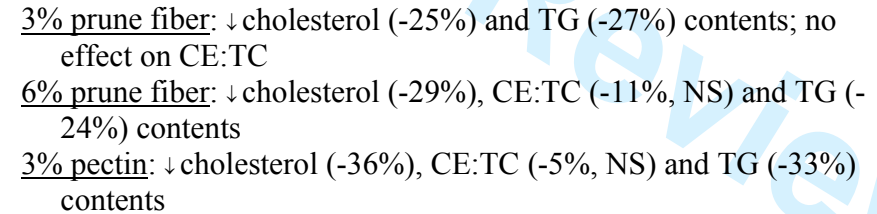 & (Tinker et al., 1994) \\
\hline $\begin{array}{l}34 \\
35\end{array}$ & Oat bran & $\begin{array}{l}\text { Rats fed high cholesterol }(1 \%+0.1 \% \text { cholic } \\
\text { acid) AIN } 76 \text { diet }\end{array}$ & $\begin{array}{l}7.5 \% \text { of NSP }+ \\
\text { lignin }\end{array}$ & 14 days & $\downarrow$ cholesterol pool $(-23 \%)$ & (Jackson et al., 1994) \\
\hline $\begin{array}{l}36 \\
37\end{array}$ & $\begin{array}{l}\text { Rice bran, oat bran, } \\
\text { or psyllium }\end{array}$ & $\begin{array}{l}\text { Rats fed } 0.25 \% \text {-cholesterol diet containing 5\% } \\
\text { cellulose }\end{array}$ & $5 \%$ of diet & 4 weeks & $\begin{array}{l}\downarrow \mathrm{TC} \text { content (resp. }-21,-41 \text { and }-47 \%) \\
\uparrow \text { bile acid synthesis (resp. }+65,+118,+60 \% \text { and no effect) }\end{array}$ & (Chezem et al., 1996) \\
\hline $\begin{array}{l}38 \\
39\end{array}$ & Guar gum & $\begin{array}{l}\text { Rats and gerbils fed high-fat }(40 \%) \text { and } 6.5 \% \\
\text { cellulose diet }\end{array}$ & $6.5 \%$ of diet & $\begin{array}{c}21 \text { (gerbils) } \\
\text { and 19 } \\
\text { (rats) davs }\end{array}$ & $\begin{array}{l}\text { Gerbils: } \downarrow \text { \TC }(-47 \%) \text { and FC }(-10 \%) \text { contents, and } \uparrow \text { TL content } \\
(+5 \%, \text { NS }) \\
\text { Rats: } \downarrow \text { TL }(-39 \%) \text { and } \uparrow \mathrm{TC}(-50 \%) \text { and FC }(0 \%) \text { contents }\end{array}$ & (Onning and Asp, 1905) \\
\hline $\begin{array}{l}40 \\
41 \\
42 \\
43 \\
44\end{array}$ & $\begin{array}{l}\text { Cellulose, guar gum, } \\
\text { pectin, konjac } \\
\text { mannan or gum } \\
\text { arabic }\end{array}$ & Rats fed high-fat ( $15 \%$ fish oil) diet & $10 \%$ of diet & $\begin{array}{l}\text { (rats) days } \\
8 \text { weeks }\end{array}$ & 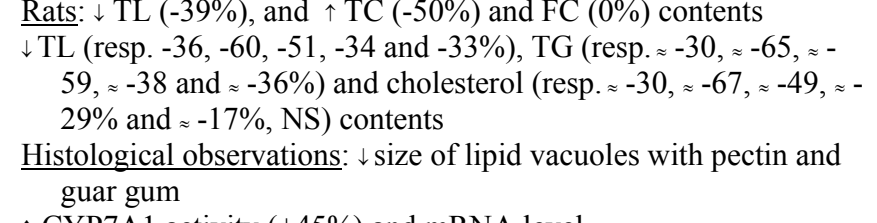 & (I Isal and I Isal, 1999) $\Downarrow$ \\
\hline 45 & Psyllium and pectin & $\begin{array}{l}\text { Male, female and ovariectomized guinea pigs } \\
\text { fed control diet }\end{array}$ & $5 \%+5 \%$ of diet & $-\mathrm{c}$ & $\uparrow$ CYP7A1 activity $(+45 \%)$ and mRNA level & (Roy et al., 2000) \\
\hline 47 & $\begin{array}{l}\text { Dietary fiber } \\
\text { complex }\end{array}$ & Rats fed AIN-76A diet containing $10 \%$ cellulose & $10 \%$ of diet & 21 days & $\downarrow$ cholesterol content $(-17 \%) ; \uparrow$ TG content $(+36 \%, \mathrm{NS})$ & (Kritchevsky and Tepper, 2005) \\
\hline $\begin{array}{l}49 \\
50\end{array}$ & $\beta$-glucan concentrate & $\begin{array}{l}\text { Rats fed modified AIN-93G diet containing } \\
0.25 \% \text { cholesterol }\end{array}$ & $5 \%$ of diet & 28 days & $\downarrow$ TC content $(\approx-30 \%)$ & (Gallaher and Plate, 2005) \\
\hline 51 & Psyllium husks & Mice fed standard AIN-93M diet & $10 \%$ of diet & $\begin{array}{l}3 \text { and } 10 \\
\text { weeks }\end{array}$ & $\begin{array}{l}\text { At week 3: Up-regulation of genes involved in fatty acid } \beta \text { - } \\
\text { oxidation (e.g. 1.6-fold for CPT1a) and down-regulation of } \\
\text { genes involved in lipid biosynthesis (e.g. 3.7-fold for SREBF }\end{array}$ & (Chan and Heng, 2008) \\
\hline
\end{tabular}




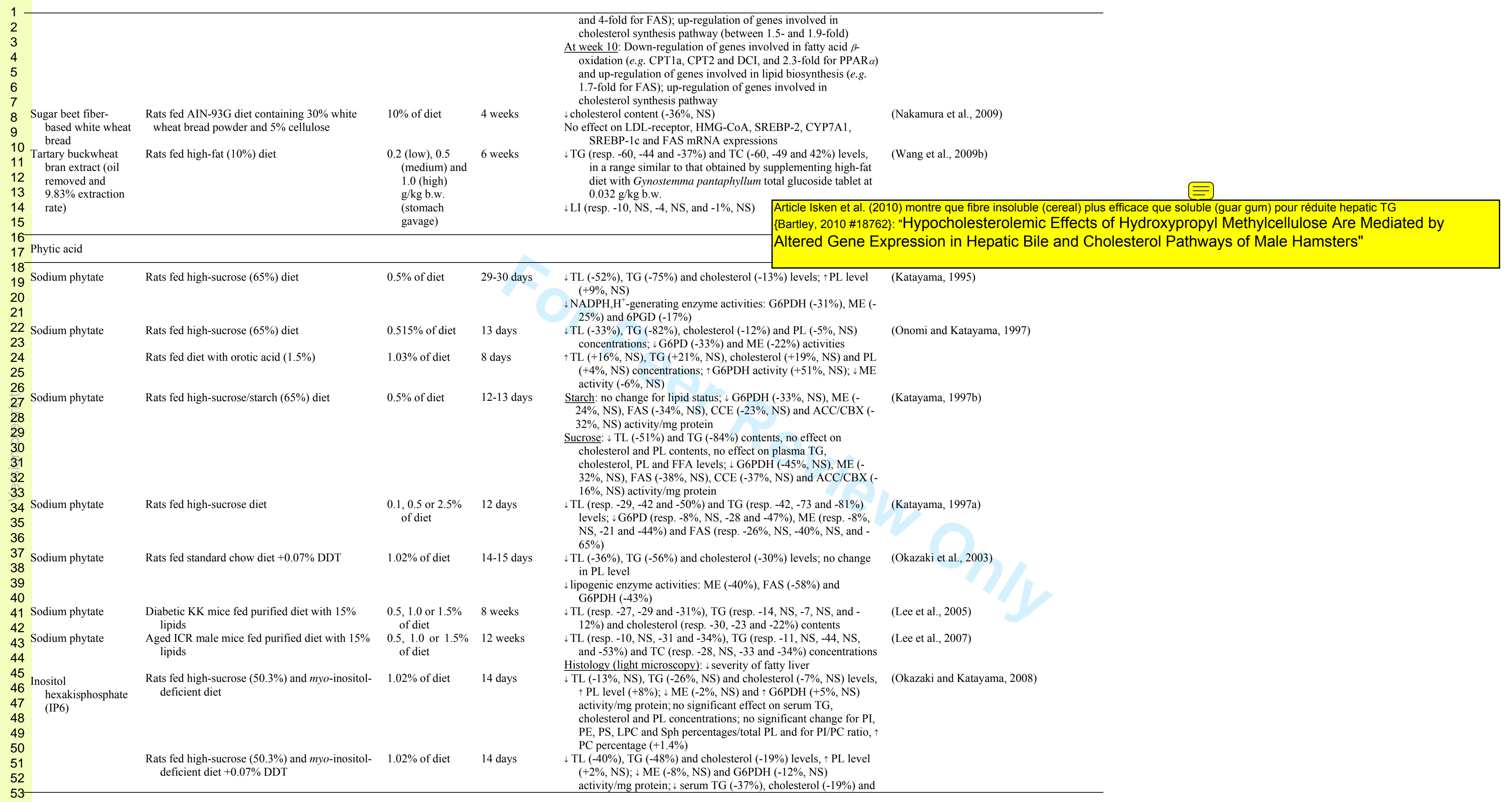




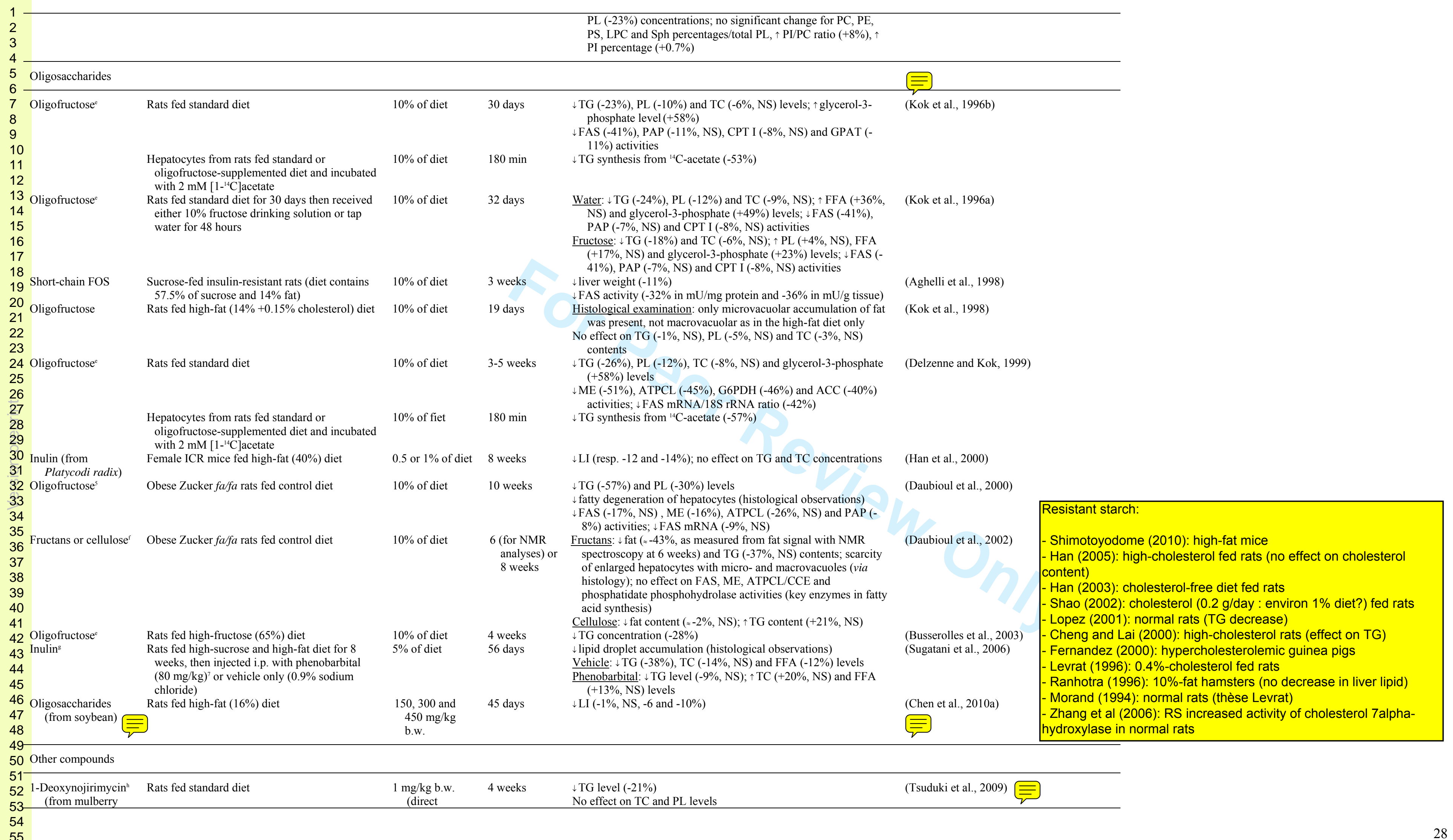




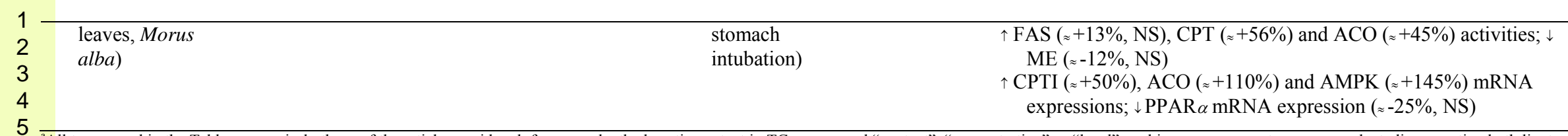

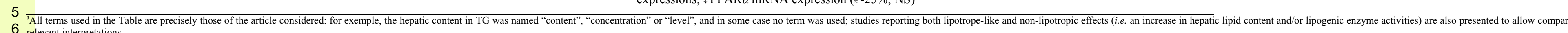

7 IIndicates the decreased or increased percentage induced by the lipotrope compared to the control, i.e. steatogen diet (NS - Not Significant - means absence of significativity for the change observed; in other cases, the effect was either significant or no information was given in the article

$8{ }^{\text {No data given in the reference }}{ }^{\circ}$ Contained oat bran, citrus pectin, guar gum, cellulose, apple pulp fiber, rice fiber, locust bean gum, date fiber, prune fiber, and fructo oligosaccharides

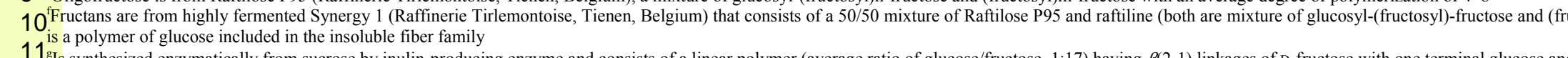

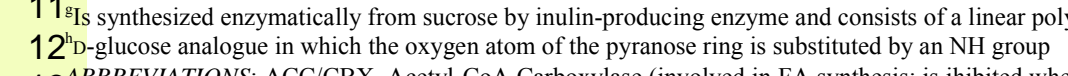

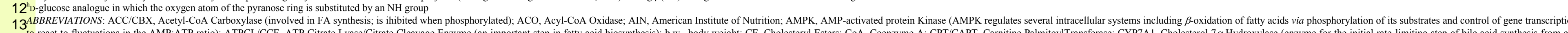

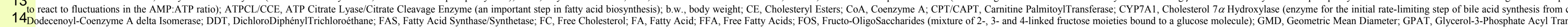

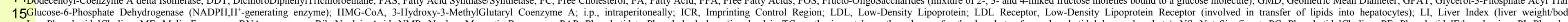

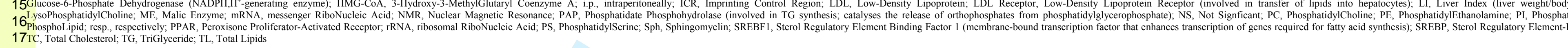
18 
Supplemental Table 4 In vivo, ex vivo and in vitro studies reporting effects on hepatic lipid metabolism following supplementation of carotenoids, pholyphenols and polyphenol-derived compounds

In vivo or in vitro modes

Supplemented Duration of

Hepatic effect(s)

References

5

exposition

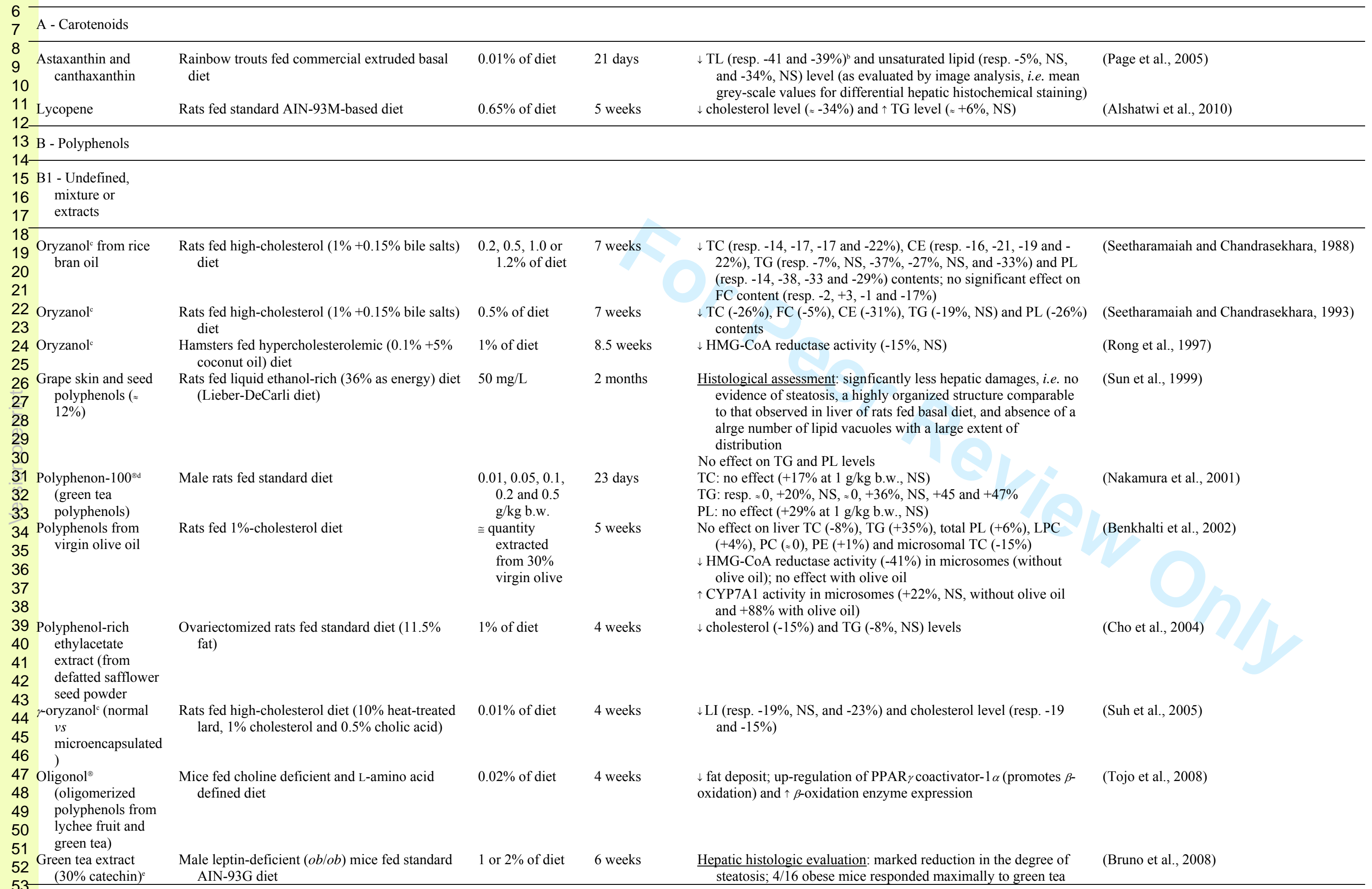
stcatosis, $4 / 16$ obese mice responded maximally to green 
11 extract, $95 \%)$

13 extract $(45 \%)$
14 from walnut

$\begin{array}{ll}14 & \text { from walnut } \\ 15 & \text { (Juglans regia L.) }\end{array}$

15
16
17

17

19

20

21

23

26 Polyphenol extrac

27 from Nelumb

nucifera leaf
28
(14.8\% phenolic

29 acids and $56 \%$

31 Silymarin (extrac

32 from milk thistle

33 seeds, Silybu

35 Polyphenol-rich

36 extract fron

37 sabdariffa $\left(\approx 74^{\circ}\right.$

38 polyphenols)

39

41

43

44
45

45
46

47 Polyphenol-rich

48 longan

49 (Dimocarpus
50 longans Lour.)

51 flower wate

52 extract

52 Anthocyanin-rich

Rats fed high-fat ( $(19 \%)$ high-sucrose $(30 \%)$
for 6 weeks, then + Provinol for 6 weeks

Mice fed high-fat (32\%) diet

50,100 or 200

$\mathrm{mg} / \mathrm{kg}$
$\mathrm{kg}$

suspended in

water and

given orally

30 or 100

10,30 or 100
$\mu \mathrm{g} / \mathrm{mL}$

10 or 100

$\mu \mathrm{g} / \mathrm{mL}$

Hamsters fed high-fat $(10 \%)$ diet containing $0.2 \%$ cholesterol

1 or $2 \%$ of diet

10 weeks

$0.01 \%$ of diet

10 weeks Male hamsters fed calorie-rich-fat $(0.2 \%$
cholesterol and $10 \%$ coconut oil $)$ diet HepG2 cells

0.1 or $0.2 \%$ of $\quad 10$ weeks

diet

$\underset{\mathrm{mg} / \mathrm{mL}}{0.1,0.5 \text { or } 1.0}$

6 hours

$\begin{array}{ccc} & \mathrm{mg} / \mathrm{mL} & \\ \text { Rats fed hypercaloric diet } & 1.25 \text { or } 2.5 \% & 9 \text { weeks }\end{array}$

(w/v) as
drinking
water

extract, resulting in grade 1 histologic score; for most, effect

Hepatic steatosis grading: 2.0 at $1 \%$ green tea extract and 2.1 at $2 \%$ (grades 1,2 and 3 correspond respectively to fatty hepatocytes occupying $<33 \%, 33-66 \%$ and $>66 \%$ of the hepatic

dose-dependently TL (resp $\approx-21$ and $\approx-39 \%$ ) and TG (resp $\approx-20$ and $\approx-41 \%$ ) concentrations; no significant effect on cholesterol concentration (resp. $z-7$ and $z+13 \%$ )

Histological examination: no preponderance of large droplets in which bulky fat vacuole distends the hepatocyte, and similar

appearance to that of control ( $4 \%$ fat)

No significant effect on cholesterol content

Tended to $\downarrow$ mitochondrial $\beta$-oxidation (resp. - $15 \%$, NS, $-29 \%$, NS and $-18 \%$, NS) and $\uparrow$ cytosolic $\beta$-oxidation (resp. $+28 \%$, NS,

$\uparrow$ PPAR $\alpha$ (resp. $\approx 1.45$-fold, NS, 1.7-fold and 1.4-fold, NS) and expression ratio vs control; no significant effect on CPT1A mRNA expression

$\uparrow$ TG accumulation within cells (rosp. $+47,+42$ a

$\uparrow$ PPAR $\alpha(\approx 1.65$-fold at $1 \mu \mathrm{g} / \mathrm{mL}$ and 1.7 -fold at $100 \mu \mathrm{g} / \mathrm{mL}$ ),
CPTA1 (resp $\approx 1.2$-fold, NS, 1.15 -fold, NS, and 4 -fold, NS) and ACOX1 (resp. $\approx$ 1.3--fold, 1.3-fold and 1.3-fold) mRNA expression ratio vs control cholesterol (resp. -27 and $-40 \%$ ) and TG (resp. -10 and $-39 \%$ ) levels

(Feillet-Coudray et al., 2009)

(Shimoda et al., 2009)

(Lin et al., 2009) number of lipid vesicles increased by the high-fat diet

cholesterol (-22\%) and TG (-25\%) levels

Histological examinations: significantly $\downarrow$ number of lipid vesicle

$\downarrow$ cholesterol (resp. $\approx-53$ and $\approx-58 \%$ ) and TG (resp. $\approx-39$ and $\approx-$ $49 \%$ ) levels $\approx-23 \%, \mathrm{NS}, \approx-48$ and $\approx-79 \%$ ) and TG (resp. $\approx-$ 4 cholesterol (resp. $z-28 \%, \mathrm{NS}, \approx-48$ and $z-79 \%)$ and TG (resp. $\approx-$
$43, z-54$ and $z-62 \%$ ) contents $43,--54$ and $z-62 \%$ ) contents
$\downarrow$ dose-dependently FAS (resp. $14,-53$ and $-75 \%$ ) and HMG-CoA reductase (resp. $-7,-46$ and $-69 \%$ ) protein expression; $\downarrow$ HMGCoA reductase (resp. $0,-75$ and $79 \%$ ) and SREBP-1c (resp. $66,-64$ and $-69 \%$ ) protein expression

AMPKphosphorylated (resp. $+49,+46$ and $+45 \%$ ), PPAR $\alpha$ (resp. $+14,+22$ and $+37 \%$; dose-dependent) and LDLR (resp. +42 ,

$\begin{array}{llll}\text { HepG2 cells } & 0.05 \text { or } 0.5 & 18 \text { hours } & \text { No effect on AMPK and } \beta \text { actin protein expression } \\ \uparrow \text { LDL uptake (resp. } z+10 \text { and } 65 \% \text { ) }\end{array}$

$\downarrow$ TG (resp. $-5 \%$, NS, and - $27 \%$ ) and cholesterol (resp. - 19 and -

(Yang et al., 2010a)

$\uparrow \operatorname{LDLR}($ resp. $z+50$ and $z+88 \%$ ), PPAR $\alpha$ (resp. $z+43$ and $z+50 \%$ )

and UCP2 (resp. $z+14 \%$, NS, and $z+16 \%$, NS) mRNA

expression; $\downarrow$ SREBP-1c (resp. 0 and $\approx-14 \%$ ) and FAS (resp. $\approx$ -

$10 \%$, NS, and $\approx-16 \%$ ) mRNA expression
TC (resp. - $7 \%$, NS, - $7 \%$, NS, and $-13 \%$ ) and TG (resp. - $8 \%$, NS, - (Hou et al., 2010) 


\begin{tabular}{|c|c|c|c|c|c|c|}
\hline & $\begin{array}{l}\text { extract (from } \\
\text { black rice) }\end{array}$ & rats & $\begin{array}{l}\mathrm{mg} \text { gkg b.w. i.g. } \\
\text { injected }\end{array}$ & & $\begin{array}{l}9 \text { and }-13 \% \text { ) levels } \\
\text { Histopathological examinations: } \text { a alterations (apparently in } \\
\text { relation with lipid accumulation) }\end{array}$ & $\equiv$ \\
\hline \multicolumn{7}{|c|}{ B2 - Phenolic acids } \\
\hline & Ferulic acid & $\begin{array}{l}\text { Rats fed high-cholesterol }(1 \%+0.15 \% \text { bile salts }) \\
\text { diet }\end{array}$ & $0.075 \%$ of diet & 7 weeks & $\begin{array}{l}\downarrow \mathrm{TG}(-19 \%, \mathrm{NS}) \text { and PL }(-23 \%) \text { contents; } \uparrow \mathrm{TC}(+1 \%, \mathrm{NS}), \mathrm{FC} \\
(+5 \%, \mathrm{NS}) \text { and } \mathrm{CE}(+1 \%, \mathrm{NS}) \text { contents }\end{array}$ & (Seetharamaiah and Chandrasekhara, 1993) \\
\hline 9 & $\begin{array}{l}\text { Ferulic acid } \\
\text { Ferulic acid } m \text { - }\end{array}$ & $\begin{array}{l}\text { Rats fed } 10 \% \text {-fat diet } \\
\text { Rats fed high-cholesterol (1\%) diet }\end{array}$ & $\begin{array}{l}0.4 \% \text { of diet } \\
0.013 .011 \text { or }\end{array}$ & $\begin{array}{l}4 \text { weeks } \\
5 \text { w weeks }\end{array}$ & $\begin{array}{l}\downarrow \mathrm{TC}(-3 \% \text {, NS) and lipid }(-9 \% \text {, NS) contents } \\
\text { No effect on TG and cholesterol contents }\end{array}$ & $\begin{array}{l}\text { (Kamal-Eldin et al., 2000) } \\
\text { (Kim et al., 2003) }\end{array}$ \\
\hline $\begin{array}{l}10 \\
11 \\
12\end{array}$ & $\begin{array}{l}\text { Ferulic acid, } m- \\
\text { hydroxycinnamic } \\
\text { acid or 3,4- } \\
\text { dihydroxyphenyl- }\end{array}$ & Rats fed high-cholesterol (1\%) diet & $\begin{array}{l}0.013,0.011 \text { or } \\
\quad 0.012 \% \text { of } \\
\text { diet }\end{array}$ & 5 weeks & $\begin{array}{l}\text { No effect on TG and cholesterol contents } \\
\downarrow \text { HMG-CoA reductase (respp } z-54, z-40 \text { and } z-51 \% \text { ) and ACAT } \\
\text { (resp; } ;-36, z-34 \text { and } z-41 \% \text { ) activities }\end{array}$ & (Kim et al., 2003) \\
\hline $\begin{array}{l}13 \\
14\end{array}$ & propionic acidi & & & & & \\
\hline $\begin{array}{l}14 \\
15\end{array}$ & Gallic acid & $\begin{array}{l}\text { FAS from chicken liver } \\
\text { Male ICR mice fed } 10^{\circ} \text { fat ( } a 1 m \text { oil) diet }\end{array}$ & $0.5 \mathrm{mM}$ & 3 hours & FAS residual activity $\approx 97 \%$ & (Wang et al., 2003) \\
\hline 16 & Ferulic acld & Male ICR mice fed $10 \%$-tat (palm oil) diet & $1 \%$ of diet & 15 days & $\begin{array}{l}\checkmark \mathrm{FAS}(z-21 \% \text {, NS), ATPCL }(z-23 \%, \mathrm{NS}), \mathrm{ME}(z 0 \%) \text { and G6PDH } \\
(z-26 \%, \text { NS) activities }\end{array}$ & (Odbayar et al., 2006) \\
\hline $\begin{array}{l}17 \\
18\end{array}$ & & & & & $\begin{array}{l}\downarrow \text { ACC }(z 0 \%) \text {, FAS }(z-10 \%, \text { NS) and ATPCL }(z-8 \%, \text { NS) mRNA } \\
\text { levels }\end{array}$ & \\
\hline 19 & & & & & $\uparrow$ SREBP-1c $(z+8 \%$, NS $)$ & \\
\hline 20 & & & & & $\downarrow \mathrm{mRNA}$ levels of proteins involved in regulation of lipogenesis: & \\
\hline 21 & & & & & 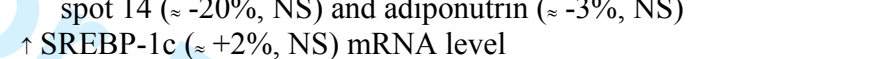 & \\
\hline 22 & Ellagic acid & HepG2 cells & 1,3 or $10 \mu \mathrm{g} / \mathrm{mL}$ & 24 hours & $\begin{array}{l}\downarrow \text { PPAR } \alpha \text { (resp. 0.59-fold, } 0.94 \text {-fold, NS, and 0.64-fold), CPT1A } \\
\text { (resp. 0.63-fold, } 0.88 \text {-fold, NS, and 0.69-fold) and ACOX1 }\end{array}$ & (Shimoda et al., 2009) \\
\hline 24 & & & & & (resp. 0.94-fold, NS, 0.63-fold and 0.60-fold) mRNA & $=1$ \\
\hline 25 & & & & & expression (vs control) & $\equiv$ \\
\hline \multicolumn{7}{|c|}{$\frac{26}{27}$ B3 - Flavonoids } \\
\hline $\begin{array}{l}28 \\
29 \\
30 \\
31\end{array}$ & $\begin{array}{l}\text { Jasmine green tea } \\
\text { epicatechins } \\
\text { (mainly EC, EGC, }\end{array}$ & $\begin{array}{l}\text { Hamsters fed hyperlipidemic (20\% fat and } 1 \% \\
\text { cholesterol) diet }\end{array}$ & $0.57 \%$ of diet & 5 weeks & $\downarrow$ TG $(-44 \%)$, FFA $(-36 \%)$ and cholesterol $(-56 \%)$ concentrations & (Chan et al., 1999) \\
\hline \multicolumn{2}{|c|}{$\begin{array}{l}32 \text { Naringin + hesperidin } \\
33\end{array}$} & Rats fed high-cholesterol (1\%) diet & $\begin{array}{l}0.05+0.05 \% \text { of } \\
\text { diet }\end{array}$ & 6 weeks & $\begin{array}{l}\downarrow \downarrow \text { cholesterol }(-28 \%) \text { and TG }(-21 \%) \text { contents } \\
\downarrow \text { HMG-CoA reductase }(-31 \%) \text { and ACAT }(-31 \%) \text { activities }\end{array}$ & (Bok et al., 1999) \\
\hline \multirow{3}{*}{$\begin{array}{l}34 \\
35 \\
36 \\
37 \\
38 \\
39 \\
40 \\
41\end{array}$} & $\begin{array}{l}\text { Soy isoflavone } \\
\text { powder }(83.3 \% \\
\text { isoffavones) }\end{array}$ & $\begin{array}{l}\text { Rats fed atherogenic diet ( } 9 \% \text { fat, } 1.2 \% \\
\quad \text { cholesterol and } 0.2 \% \text { cholic acid) }\end{array}$ & $20 \%$ of diet & 63 days & $\begin{array}{l}\downarrow \text { TG }(-33 \%) \text { and } \uparrow \text { TC }(+10 \%, \mathrm{NSS}) \mathrm{CE}(+9 \%, \mathrm{NS}) \text { and } \\
\quad \text { unesterified cholesterol }(+17 \%) \text { concentrations }\end{array}$ & (Peluso et al., 2000) \\
\hline & $\begin{array}{l}\text { Epigallocatechin } \\
\text { gallate (EGCG) }\end{array}$ & FAS from chicken liver & $\approx 27-110 \mu \mathrm{M}$ & $60 \mathrm{~min}$ & $\downarrow$ FAS activity (reversible fast-binding inhibition): $\mathrm{IC}_{50}=52 \mu \mathrm{M}$ & (Wang and Tian, 2001) \\
\hline & Tannic acid & Male rats fed standard diet & $\begin{array}{l}0.1,0.2,0.5 \text { and } \\
1.0 \mathrm{~g} / \mathrm{kg} \text { b.w. }\end{array}$ & 23 days & $\begin{array}{l}\text { TC: no effect } \\
\text { TG: resp. }+34,+38, \approx 0 \text { and }+47 \% \\
\text { PL: resp. }+17 \%, N S,+18 \%, N S,+33 \%,+29 \%, \text { NS }\end{array}$ & (Nakamura et al., 2001) \\
\hline \multirow{7}{*}{$\begin{array}{l}42 \\
43 \\
44 \\
45 \\
46 \\
47 \\
48 \\
49 \\
50 \\
51 \\
52\end{array}$} & $\begin{array}{l}\text { Hesperetin (from } \\
\text { citrus) }\end{array}$ & Rats fed $1 \%$-orotic acid diet containing $10 \%$ fat & $1 \%$ of diet & 10 days & $\begin{array}{l}\downarrow \text { microsomal PAP }(z-30 \%) \text {, G6PDH }(z-44 \%), \mathrm{ME}(z-41 \%) \text { and } \\
\text { DGAT }(z-48 \%) \text { activities }\end{array}$ & (Cha et al., 2001) \\
\hline & $\begin{array}{l}\text { Naringenin or } \\
\text { hesperetin }\end{array}$ & HepG2 cells & $\begin{array}{l}10-200 \text { or } 50- \\
200 \mu \mathrm{M}\end{array}$ & 24 hours & $\begin{array}{l}\downarrow \text { dose-dependently ApoB accumulation into the media: } \\
\text { Naringenin: from } \approx-7 \%(10 \mu \mathrm{M}) \text {, NS, to } \approx-83 \%(200 \mu \mathrm{M})\end{array}$ & (Wilcox et al., 2001) \\
\hline & Taringenin & $\begin{array}{l}\text { HepG2 cells pre-incubated } 24 \mathrm{~h} \text { with flavonoid } \\
\text { and incubated } 20 \mathrm{~min} \pm 0.1 \mathrm{mM} \text { oleate }\end{array}$ & 50 or $200 \mu \mathrm{M}$ & $\begin{array}{l}24 \text { hours }(+20 \\
\min )\end{array}$ & 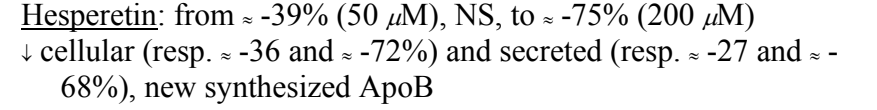 & \\
\hline & $\begin{array}{l}\text { aringenin or } \\
\text { hesperetin }\end{array}$ & HepG2 cells & 50 or $200 \mu \mathrm{M}$ & 24 hours & $\checkmark$ cellular CE mass: & \\
\hline & hesperetin & & & & $\begin{array}{l}\text { Nariningenin: resppp } z-8 \%, \text {, NS, and } z-26 \% \\
\text { Hesperetin: resp. } z-17 \%, \text { NS, and } z-21 \%\end{array}$ & \\
\hline & & & & & $\begin{array}{l}\text { ce cellular FC mass: } \\
\text { Naringenin: resp. } z+4 \% \% \text {, NS, and } z+7 \% \text {, NS }\end{array}$ & \\
\hline & & & & & $\begin{array}{l}\text { Hesperetin: resp. } \approx+3 \% \%, \text { NS, and } \approx+3 \%, \text { NS } \\
\uparrow \text { cellular TG mass: }\end{array}$ & \\
\hline
\end{tabular}


oleate into CE (resp. -37 an

(resp. $+20 \%$, NS, and $+16 \%$ presence ceetlon

50,100 or 200

inding (resp. $\approx 0$ and $\approx$ 


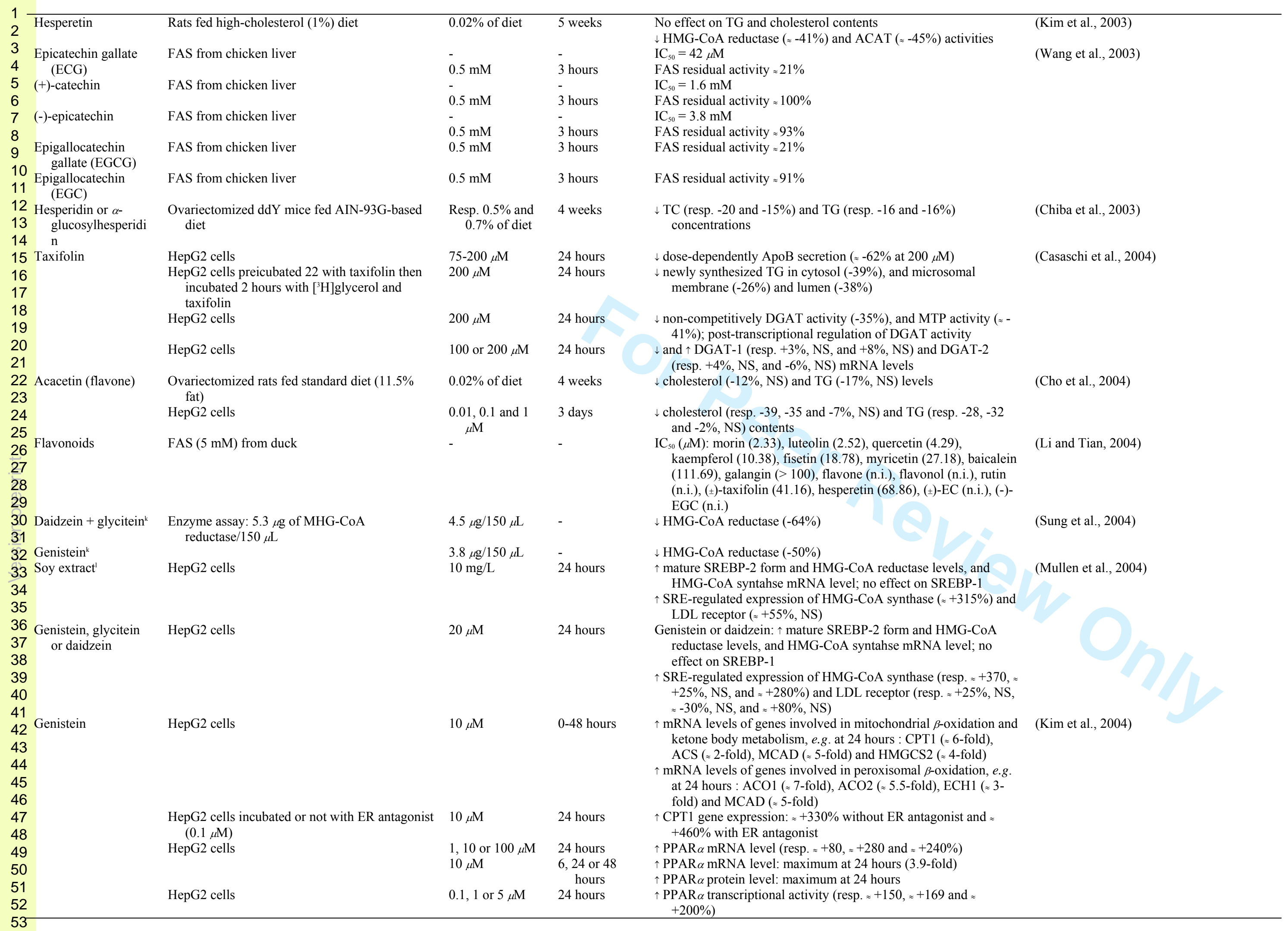




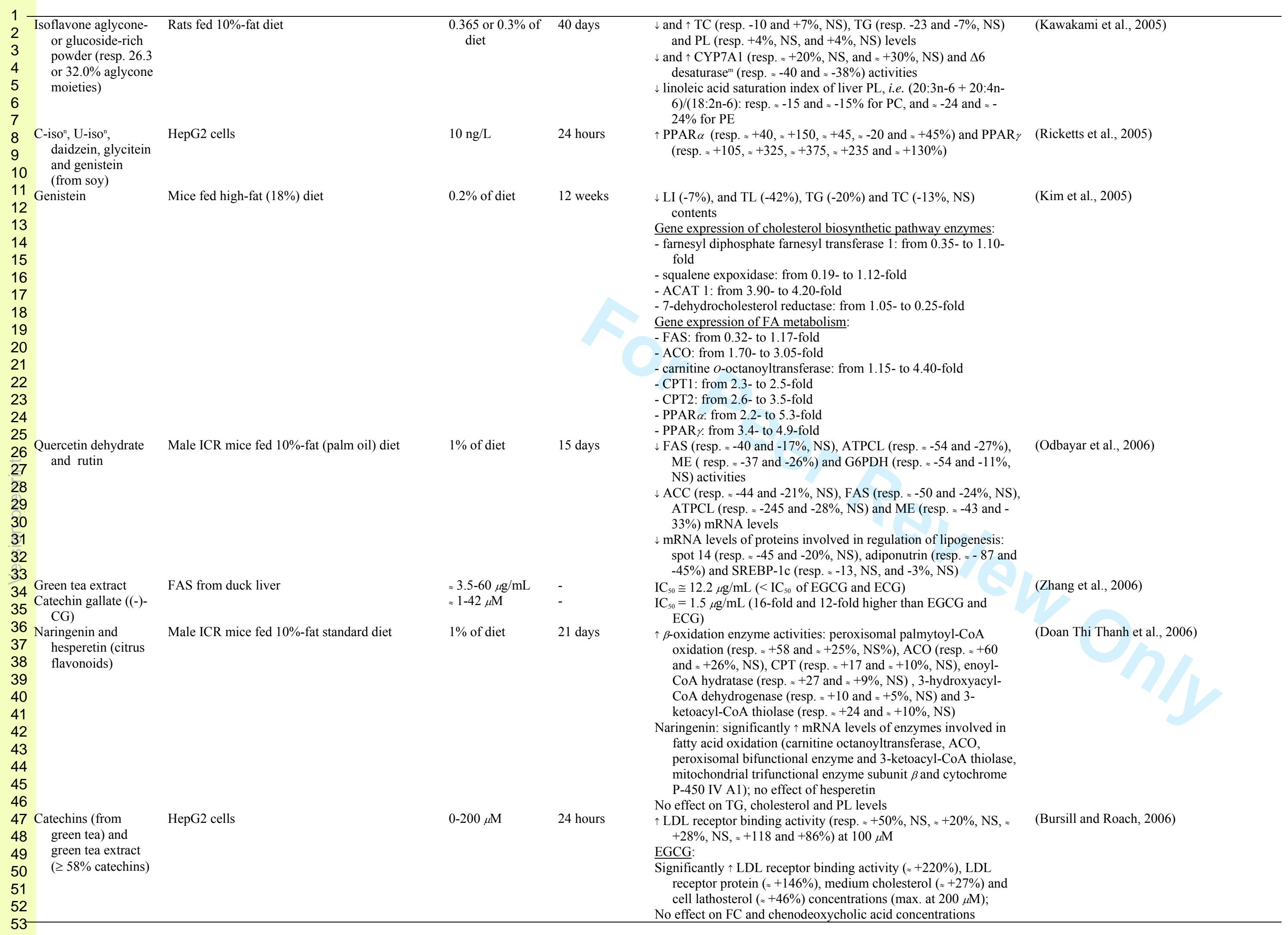


Three-, 24 or 27-28-months old rats fed standard $160 \mathrm{mg} / \mathrm{kg}$ b.w. diet with $700 \mu \mathrm{L}$ ethanol/kg b.w.

$160 \mathrm{mg} / \mathrm{kg}$ b.w.

$160 \mathrm{mg} / \mathrm{kg}$ b.w.
$500 \mu \mathrm{g} / \mathrm{mL}, 30$ $\mu \mathrm{M} / \mathrm{mL}, 30$ Chamiloflan, AP7Glu Hepatocytes isolated from 90- and 720-day-old 13 (-)-epigallocatechin-

ice fed high-fat $(34.9 \%)$ diet

$0.32 \%$ of diet

16 weeks

$\downarrow$ TC concentration $(\approx-28 \%)$

active transcription factor form of SREBP- $1(z+42-56 \%$, from nuclear cell fraction) and inactive precursor form of SREBP(from membrane fraction) to undetectable level.

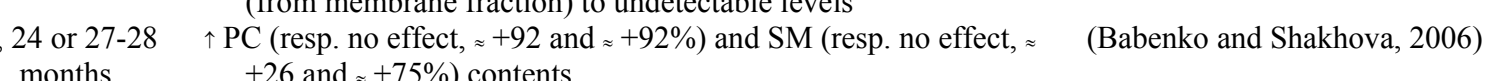

ceramide (precursor of SM) content (resp. no effect, $\approx-46$ and $\approx-$

$70 \%)$

$\downarrow$ ceramide production from [ $\left[{ }^{4} \mathrm{C}\right] p$ palmitic acid pre-labeled Sph $(-$

$28 \%$; no effect on sphingoside production

(a)

$\downarrow$ LI (-22\%), fatty liver incidence (from 21/22 mice in high-fat group to $4 / 22$ mice in high-fat + EGCG-supplemented group and

TG content (-69\%)
Gross examination: around 3-fold less in size/volume and similar Histological group (4.3\% fat)

25, 50 and 10030 days $\quad \begin{gathered}\text { accumulation similar to control group (4.3\% fat) } \\ \text { dose-dependently TC (resp. - } 14 \% \text {, NS, - } 20 \text { and }-31 \% \text { ) and FFA }\end{gathered} \quad$ (Guo et al., 2009)

$\mathrm{mg} / \mathrm{kg}$ b.w. $\quad$ (resp. $-15 \%$, NS, -32 and $-37 \%$ ) concentrations, and $\downarrow$ TG

concentration $(-11 \%, \mathrm{NS})$ at the dose of $100 \mathrm{mg} / \mathrm{kg} ; \uparrow$ TG

$\mathrm{mg} / \mathrm{kg}$.

Morphological evaluation: fom $7 / 10$ rats with severe steatosis

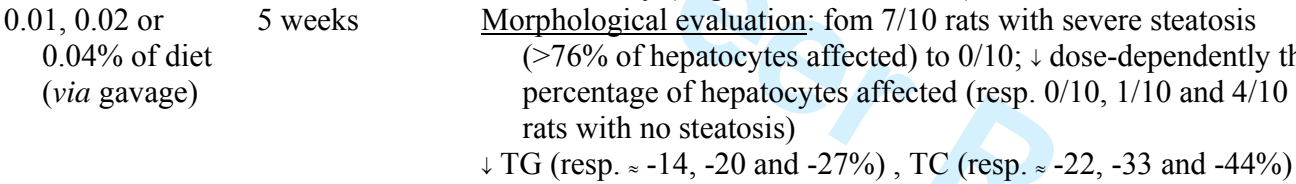

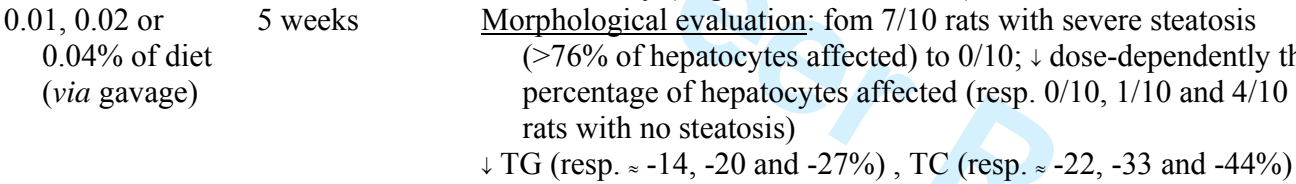

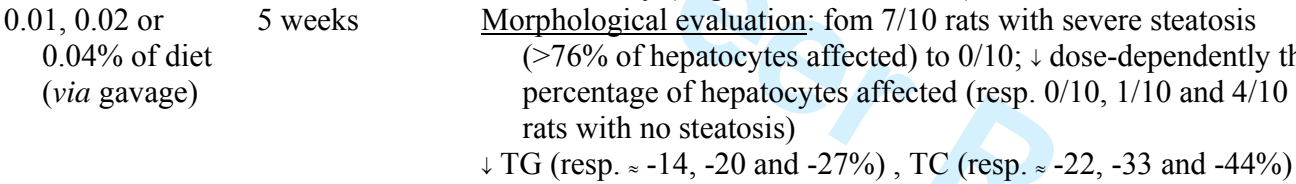
and FFA (resp. $\approx-20,-41$ and $-62 \%$ ) contents
$\uparrow$ PPAR $\alpha$ protein expression $(z+89 \%)$

$1 \mathrm{mg} / \mathrm{kg}$ b.w. $\quad 26$ weeks $\quad \uparrow$ PPAR $\alpha$ gene expression $(z+160 \%)$; no effect on CPT-1, ACO,

SREBP-1, MCD, FAS and ACC gene expressions
No effect on TG content

Rats fed high-fat $(\approx 15 \%)$ diet

administered

in drinking
water (as

$100 \%$ of fluid

intake)
1,3 or $10 \mu \mathrm{g} / \mathrm{mL} \quad 24$ hours

HepG2 cells

$\downarrow$ PPAR $\alpha$ (resp. 0.60-fold, 0.58-fold, and 0.82-fold), CPT1A (0.63fold at $1 \mu \mathrm{g} / \mathrm{mL}$ and 0.74 -fold at $3 \mu \mathrm{g} / \mathrm{mL})$ and ACOX1 $(0.63-$ fold at $1 \mu \mathrm{g} / \mathrm{mL}$ and 0.82 -fold at $3 \mu \mathrm{g} / \mathrm{mL}) \mathrm{mRNA}$ expression
(vs control), and $\uparrow \operatorname{PPAR} \alpha(1.31$-fold $)$ and ACOX1 (120-fold $)$ mRNA expression at $10 \mu \mathrm{g} / \mathrm{mL}$

1,3 or $10 \mu \mathrm{g} / \mathrm{mL} 24$ hours $\quad \uparrow \operatorname{PPAR} \alpha(1.08$-fold at $3 \mu \mathrm{g} / \mathrm{mL}$, NS, and 1.14 -fold at $10 \mu \mathrm{g} / \mathrm{mL}$, NS), CPT1A (resp. 1.02-fold, NS, 1.09-fold, NS, and 1.23-fold and ACOX1 (resp. 1.12-fold, NS, 1.33-fold and 1.69-fold)

mRNA expression (vs control); $\downarrow$ PPAR $\alpha$ mRNA expression at
$1 \mu \mathrm{g} / \mathrm{mL}$ (0.84-fold)

No effect on PPAR $\alpha$ mRNA expression; $\uparrow$ CPT1A (resp. 1.42-fold, 1.56 -fold and 1.42-fold) and ACOX1 (1.13-fold, NS, at 3 and
$10 \mu \mathrm{g} / \mathrm{mL}$ ) mRNA expression (vs control); $\downarrow$ ACOX1 mRNA $10 \mu \mathrm{g} / \mathrm{mL}) \mathrm{mRNA}$ expression (vs
expression at $1 \mu \mathrm{g} / \mathrm{mL}$ (0.79-fold)

1,3 or $10 \mu \mathrm{g} / \mathrm{mL} \quad 24$ hours expression at $1 \mu \mathrm{g} / \mathrm{mL}$ ( 0.79 -fold)
TG (resp. -72 and $-72 \%$ ), TC (resp. $-12 \%$, NS, and $-8 \%, \mathrm{NS}$ ), FC

0.5 or $1.0 \%$ of $\quad 6$ week

(resp. - $6 \%$, NS, and - $19 \%$, NS) and CE (resp. - $16 \%$, NS and

SREBP 1c (resp. $z-50$ and $z-75 \%$ ), FAS (resp. $z-50$ and $z-68 \%$ ), SCD1 (resp. $z-48$ and $z-62 \%$ ), HMG-CoA reductase (resp. $z$ - 


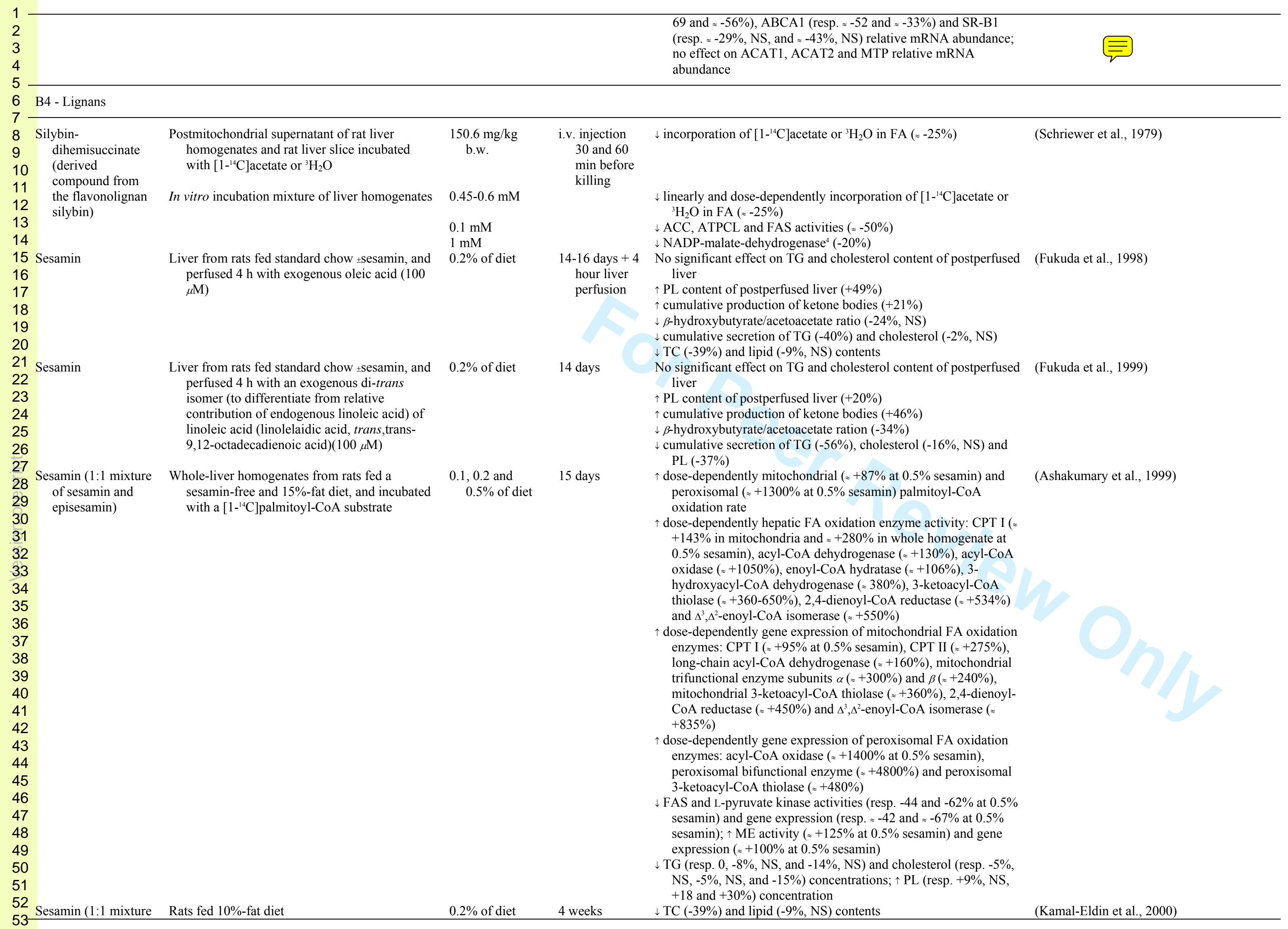


dose-dependently hepatic FA oxidation enzyme activity:

peroxisomal oxidation (resp. +22 and $+130 \%$ ), acyl-CoA

oxidase (resp. +38 and $+112 \%$ ), CPT ( (esp. +61 and $+135 \%$ ), $3-$

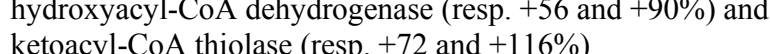

dose-dependently ACC, FAS, ATPCL, G6PDH

kinase activities (resp. $-36,-32,-30,-42$ and $-19 \%$ at $0.1 \%$, an resp. $-57,-46,-47,-59$ and $-44 \%$ at $0.2 \%$ sesamin) and gene expression (resp. $-35,-36,-28,-36$ and $-25 \%$ a $0.1 \%$, and res activity (resp. -6 and $+13 \%$ ) and gene expression (resp on +2 and 7\%)

$\downarrow$ dose-dependently activity of hepatic enzymes involved in cholesterol synthesis: farnesyl pyrophosphate synthase (resp. -
20 and $-29 \%$ ) and squalene synthetase (resp. -32 and $-39 \%$ ); no 20 and $-29 \%$ ) and squalene synthetase (resp. -32 and $-39 \%$.
effect on HMG-CoA synthase

$\downarrow$ dose-dependently gene expression (mRNA levels) of hepatic

enzymes involved in cholesterol synthesis: HMG-CoA

reductase (resp. -23 and $-30 \%$ ), farnesyl pyrophosphate
synthase (resp. -21 and $-35 \%$ ), squalene synthetase (resp. -30 synthase (resp. -21 and $-35 \%$ ), squalene synthetase (resp. -30
and $-44 \%$ ) and of LDL receptor (resp. - 22 and $-28 \%$ ); $\uparrow$ mRNA level of HMG-CoA synthase (resp. $+9 \%$, NS, and $+31 \%$ )
mRNA level of SREBP-1 (resp. $z-30$ and $-35 \%$ )

dose-dependently protein level of precursor and mature forms of SREBP-1 (resp. $z-13 \%$, NS, and $-37 \%$ )

dose-dependently hepatic FA oxidation enzyme activity:

peroxisomal oxidation (resp. +207 and $+600 \%$ ), acyl-CoA
oxidase (resp. +260 and $+768 \%$ ), CPT (resp +127 and $+232 \%$ ), 3-hydroxyacyl-CoA dehydrogenase (resp. +148 and $+329 \%$ ) and 3-ketoacyl-CoA thiolase (resp. +139 and $+275 \%$ )

$\downarrow$ ACC, FAS, ATPCL, G6PDH and pyruvate kinase activities (resp.
$-44,-47,-43,-60$ and $-50 \%$ at $0.2 \%$ sesamin, and resp. $-41,-39$, $-46,-55$ and $-56 \%$ at $0.4 \%$ sesamin) and gene expression (resp. $-49,-59,-44,-48$ and $-66 \%$ at $0.2 \%$, and resp. $-47,-57,-40$, sesamin $\uparrow$ dose-dependently ME activity (resp $+24 \%, \mathrm{NS}$, and $+97 \%$ ) and gene expression (resp. $+16 \%$, NS, and $+92 \%$ ) activity of hepatic enzymes involved in cholesterol synthesis: and $37 \%$ at $02 \%$ sest $0.4 \%$ sesamin and no signd squalene synthetase $(-$ sesamin); $\uparrow$ dose-dependently HMG-CoA synthase activity (resp. +66 and $+189 \%$ )

dose-dependently gene expression (mRNA levels) of HMG-CoA reductase (resp. -26 and $-42 \%)$, farnesyl pyrophosphate
synthase $(-37 \% \%$ at $0.2 \%$ sesamin), squalene synthase $(-34 \%$ ynthase (-37\% at $0.2 \%$ sesamin), squalene synthase $(-34 \%$ at dose-dependently mRNA level of HMG-CoA synthase $(+172 \%$ at $0.4 \%$ sesamin); no effect on mRNA level of farnesyl

pyrophosphate synthase at $0.4 \%$ Sesamin
dose-dependently mRNA level of SREBP-1 (resp. $\approx-37$ and -

protein level of
84 and $-88 \%$

Rats fed sesamin-free and $10 \%$-fat diet

$0.2 \%$ of diet

15 days

FA oxidation enzyme activity: mitochondrial (resp. +73 and

(Kushiro et al., 2002)

(Ide et al., 2001) 


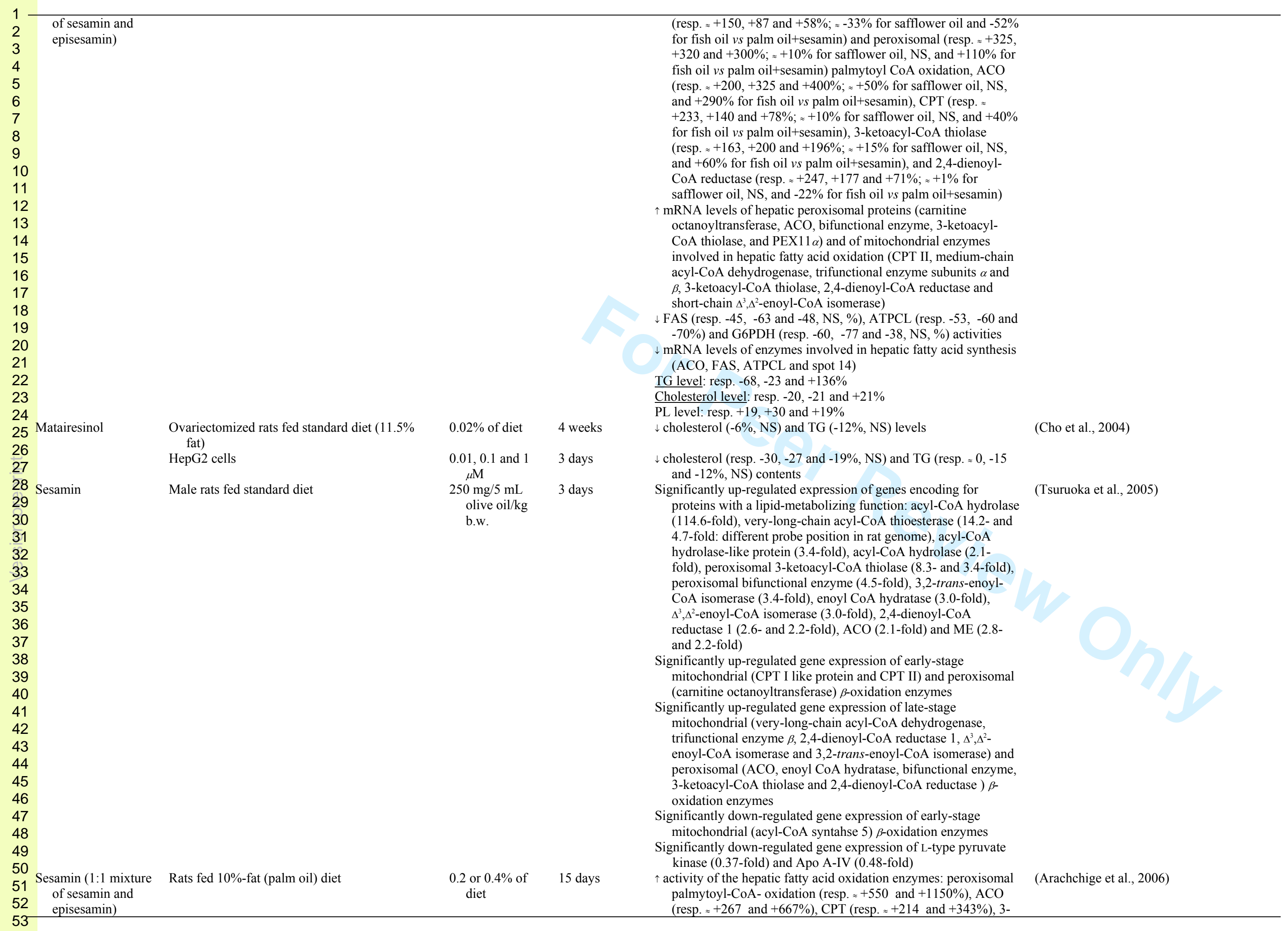


Rats fed $10 \%$-fat $(8 \%$ palm oil $+2 \%$ DHA ethyl $\quad 0.2 \%$ of diet ester) diet

Rats fed 10\%-fat (palm oil) diet

$0.2 \%$ of diet

hydroxyacyl-CoA dehydrogenase (resp. $z+175$ an 26

se (resp. $z+146$ and $+242 \%$ )

tivity of the hepatic FA oxidation enzymes: peroxisomal

+211\%), 3-hydroxyacyl-CoA dehydro ACO $(z+575 \%)$, CPT (

ketoacyl-CoA thiolase $(z+151 \%)$ -

ctivity of the hepatic FA oxidatio

palmytoyl-CoA- oxidtion $\left(z+300^{\circ}\right)$ ymes: peroxisomal

$250 \%$ ), enoyl-CoA hydratase $(z+48 \%)$, 3-hydroxyacyl-CoA

dehydrogenase $(z+141 \%)$ and 3 -ketoacyl-CoA thiolase $(z$

ctivity of the hepatic FA oxidation enzymes: peroxisomal

Rats fed $10 \%$-fat ( $8 \%$ palm oil + $2 \%$ EPA ethyl $\quad 0.2 \%$ of diet

15 days $+140 \%$ ), enoyl-CoA hydratase $(z+73 \%)$, 3-hydroxyacyl-CoA dehydrogenase $(z+188 \%)$ and 3 -ketoacyl-CoA thiolase $(z$

$+333 \%)$

4 experiments:

Is of hepatic peroxisomal proteins (carnitine

CoA thiolase, and PEX11 bifunctional enzyme, 3-ketoacyl-

involved in hepatic fatty acid oxidation (CPT II, medium-cha

acyl-CoA dehydrogenase, trifunctional enzyme subunits $\alpha$ and

$\beta, 3$-ketoacyl-CoA

Rats fed 10\%-fat (palm oil) diet

0.06 or $0.2 \%$ of $\quad 10$ days

peroxisomal palmytoyl-CoA oxidation (resp. +8 , NS, and $+46 \%$

and ACO (resp. +8 , NS, and $+31 \%$ ), CPT (resp. +31 and
$+88 \%$ ), enoyl-CoA hydratase $(-3 \%$, NS at $0.06 \%$ sesamin

$+32 \%$ at $0.2 \%$ sesamin), 3-hydroxyacyl-CoA dehydrogenase

(resp. +28 and $+89 \%$ ), 3-ketoacyl-CoA thiolase (resp. $+12, \mathrm{NS}$

$+65 \%)$ activities

FAS (resp. - 41 and - $60 \%$ ), ATPCL (resp. - 38 and -57\%), G6PDH (resp. -49 and - $64 \%$ ) and pyruvate kinase (resp. - $15 \%$, NS, and -

$39 \%$ ) activities
TG (resp. -59 and $-64 \%$ ) and cholesterol (resp. -25 and $-25 \%$ )

peroxisomal palmytoyl-CoA oxidation (resp. +51 and $+321 \%$ ),

and ACO (resp. +59 and $+220 \%$ ), CPT (resp. +64 and $+279 \%$

enoyl-CoA hydratase (resp. +24 and $+100 \%$ ), 3-hydroxyacyl-

CoA dehydrogenase (resp. 68 and $+228 \%$ ), 3-ketoacyl-CoA (hiolase 57 . $157 \%$ (57) (resp. +57 and $+15 \%$ ) activities (resp. -35 and $-67 \%$ ), G6PDH (resp. -51 and $-68 \%$ ) and pyruvate kinase (resp. -20 and $-51 \%$ ) activities

TG (resp. -18 and $-30 \%$ ) and cholesterol (resp. -17 and $-30 \%$ )

levels; $\uparrow$ PL level (resp. $+6 \%$, NS, and $+37 \%$

$0.14+0.06 \%$ of $\quad 10$ days $\quad \uparrow$ peroxisomal palmytoyl-CoA oxidation $(+148 \%)$, and ACO hydroxyacyl-CoA dehydrogenase (+156\%), 3-ketoacyl-CoA thiolase $(+139 \%)$ and 2,4-dienoyl-CoA reductase $(+101 \%)$

activities
FAS (-56\%), ATPCL (-56\%), G6PDH (-67\%) and pyruvate

Kinase $(-45 \%)$ activitie

$(-23 \%)$ levels; $\uparrow$ PL level $(+42 \%)$

mRNA abundance of enzymes involved in FA oxidation (from 政 


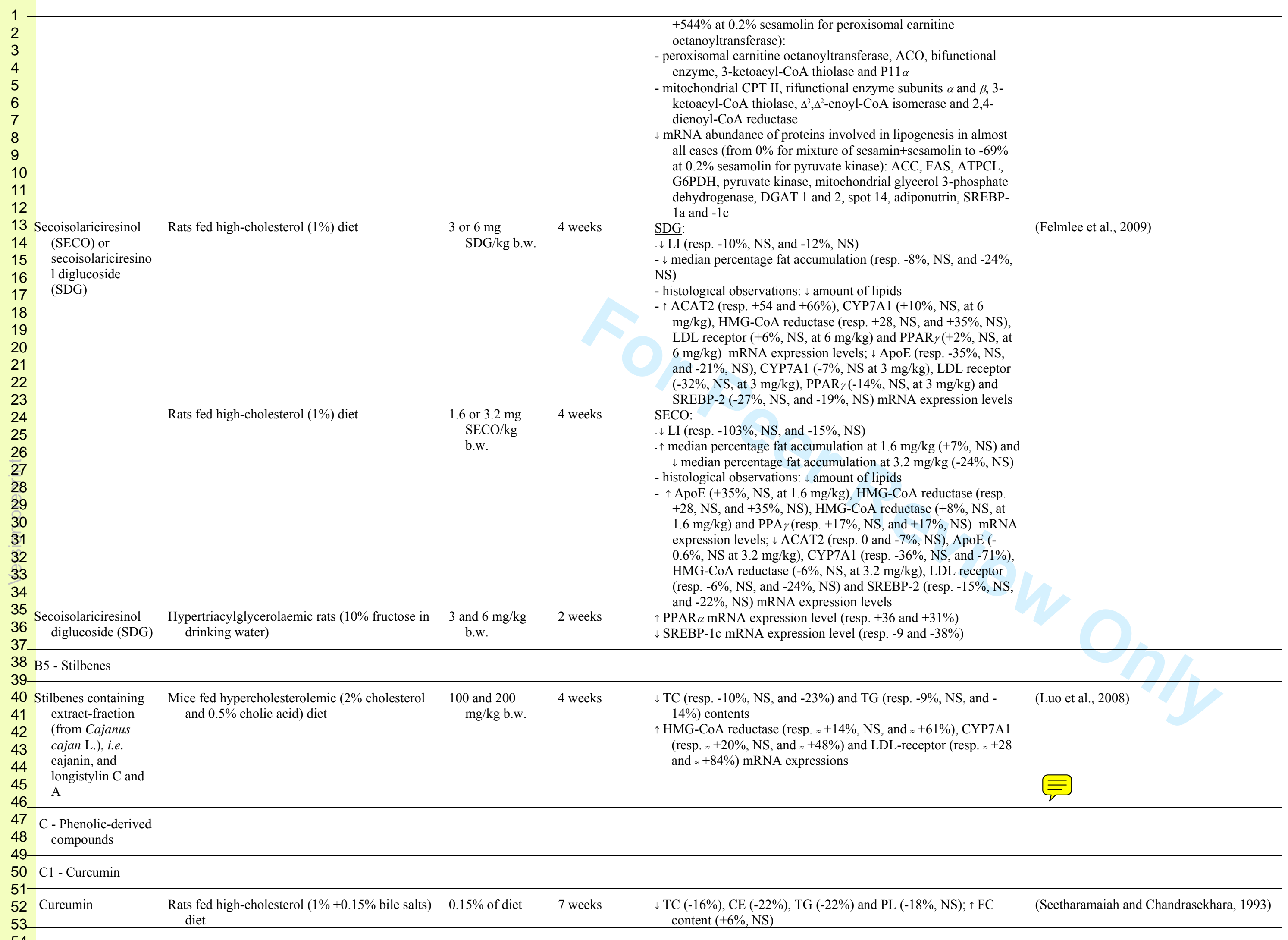




\begin{tabular}{|c|c|c|c|c|c|c|}
\hline & Curcumin & Rats fed $10 \%$-fat diet & $0.2 \%$ of diet & 4 weeks & $\downarrow \mathrm{TC}(-37 \%)$ and lipid $(-4 \%, \mathrm{NS})$ contents & (Kamal-Eldin et al., 2000) \\
\hline & C2 - Saponins & & & & & \\
\hline $\begin{array}{l}5 \\
6 \\
7 \\
8 \\
9 \\
10 \\
11 \\
12 \\
13\end{array}$ & $\begin{array}{l}\text { Ginsenosides }\left(\mathrm{Rb}_{1},\right. \\
\mathrm{Rc}, \mathrm{Rg}_{1}, \mathrm{Rd} \text { and } \\
\mathrm{Re}) \text { prufied from } \\
\text { ginseng (Panax } \\
\text { ginseng) }\end{array}$ & $\begin{array}{l}\text { Rats injected with }{ }^{14} \mathrm{C} \text {-acetate from } 30 \text { to } 120 \\
\text { min before killing }\end{array}$ & $\begin{array}{l}5 \mathrm{mg} \text { injected i.p. } \\
\text { before killing }\end{array}$ & 4 hours & 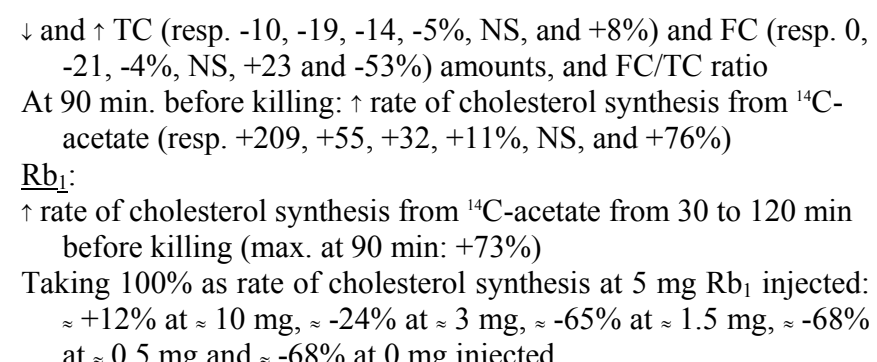 & (Sakakibara et al., 1975) \\
\hline $\begin{array}{l}14 \\
15 \\
16 \\
17\end{array}$ & $\begin{array}{l}\text { Purified saponosides } \\
\text { from Aralia } \\
\text { mandshurica } \\
\text { (mixture of } 9\end{array}$ & $\begin{array}{l}\text { Rats fed fatty ( } 40 \% \text { margarine and } 2 \% \\
\text { cholesterol) diet (with } 0.01 \% \text { methylthiouracil) }\end{array}$ & $\begin{array}{l}0.005 \text { or } 0.01 \\
\mathrm{~g} / \mathrm{kg} \text { b.w. }\end{array}$ & 12 weeks & $\begin{array}{l}0.005 \mathrm{~g} / \mathrm{kg}: \downarrow \text { and } \downarrow \mathrm{\downarrow TL}(+8 \%), \mathrm{TG}(-40 \%) \text { and TC }(+14 \%) \text { contents } \\
0.01 \mathrm{~g} / \mathrm{kg}: \downarrow \mathrm{TL}(-35 \%), \mathrm{TG}(-35 \%) \text { and TC }(-11 \%) \text { contents }\end{array}$ & (Wojcicki et al., 1977) \\
\hline $\begin{array}{l}18 \\
19 \\
20 \\
21 \\
22 \\
23 \\
24\end{array}$ & $\begin{array}{l}\text { oleanosides) } \\
\text { Commercial white } \\
\text { saponins } \\
\text { (probably from } \\
\text { European } \\
\text { Soapwort, } \\
\text { Saponaria } \\
\text { officinalis) }\end{array}$ & Rats fed normal or high-cholesterol (1\%) diet & $1 \%$ of diet & 3 weeks & $\begin{array}{l}\downarrow \text { cholesterol (resp. - } 7 \% \text {, NS, and }-52 \% \text { ) and TG (resp. - } 20 \text { and - } \\
39 \% \text { ) concentrations }\end{array}$ & (Oakenfull et al., 1979) \\
\hline $\begin{array}{l}25 \\
26 \\
27 \\
28 \\
29 \\
30\end{array}$ & $\begin{array}{l}\text { Commercial white } \\
\text { saponins } \\
\text { (probably from } \\
\text { European } \\
\text { Soapwort, } \\
\text { Saponaria } \\
\text { officinalis) }\end{array}$ & $\begin{array}{l}\text { Rats fed standard diet containing methionine- } \\
\text { supplemented sodium isolates of soybean or } \\
\text { casein ( } 25 \% \text { energy) }\end{array}$ & $1 \%$ of diet & 56 days & $\begin{array}{l}\text { Soybean-based diet: } \uparrow \text { cholesterol content }(+41 \%) \\
\text { Casein-based diet: } \downarrow \text { cholesterol content }(-4 \%)\end{array}$ & (Pathirana et al., 1980) \\
\hline $\begin{array}{l}31 \\
32 \\
33 \\
34 \\
35\end{array}$ & Saponins (purified) & $\begin{array}{l}\text { Laying hens (brown and white Leghorn) fed } \\
\text { standard diet }\end{array}$ & $\begin{array}{l}0.1 \text { or } 0.5 \% \text { of } \\
\text { diet } \\
0.1,0.2 \text { or } 0.4 \% \\
\text { of diet } \\
0.1,0.2 \text { or } 0.4 \% \\
\text { of diet }\end{array}$ & $\begin{array}{l}5 \text { or } 8 \text { weeks } \\
8,7 \text { or } 6 \\
\text { weeks } \\
18 \text { weeks }\end{array}$ & $\begin{array}{l}\downarrow \text { lipid content (resp. }-16 \% \text {, NS, and }-26 \%) \\
\text { No effect on cholesteroo content (resp. }-3 \% \text {, NS, and }+8 \% \text {, NS) } \\
\downarrow \text { lipid content (resp. } 0,-11 \%, \text { NS, and }-19 \%, \text { NS) } \\
\downarrow \text { lipid content (resp. }-15 \%, \text { NS, }-21 \text { and }-29 \% \text { ) }\end{array}$ & (Whitehead et al., 1981) \\
\hline $\begin{array}{l}36 \\
37 \\
38 \\
39 \\
40 \\
41\end{array}$ & $\begin{array}{l}\text { Steroid saponins } \\
\text { (from Gypsophila } \\
\text { plant roots) }+ \\
\text { citrus pectin } \\
\text { washed with } \\
\text { acidified ethanol }\end{array}$ & $\begin{array}{l}\text { Rats fed standard diet } \pm \text { citrus pectin washed } \\
\text { with acidified ethanol }\end{array}$ & $\begin{array}{l}0.2 \%+10 \% \text { of } \\
\text { diet }\end{array}$ & 5 weeks & 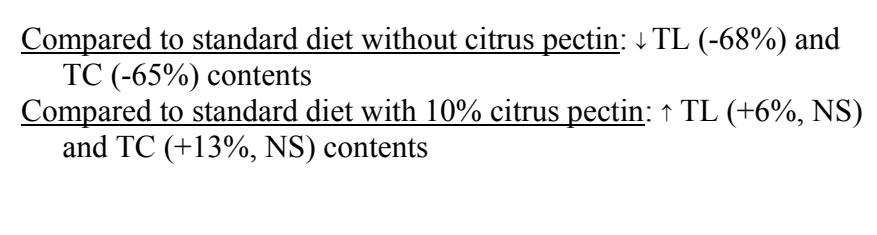 & (Rotenberg and Eggum, 1986) \\
\hline $\begin{array}{l}42 \\
43 \\
44\end{array}$ & $\begin{array}{l}\text { Mixture of } \\
\text { avecanosides A } \\
\text { and B (from oat } \\
\text { meal) }\end{array}$ & $\begin{array}{l}\text { Rats and gerbils fed high-fat }(40 \%) \text { and } 6.5 \% \\
\text { ethanol-extracted oatmeal diet }\end{array}$ & $0.07 \%$ of diet & $\begin{array}{l}21 \text { (gerbils) } \\
\text { and 19) } \\
\text { (rats) days }\end{array}$ & $\begin{array}{l}\text { Gerbils: } \downarrow \text { TL }(-4 \%, \text { NS), TC ( }(-6 \%, \text {, NS) and FC }(-6 \%, \text { NS) contents } \\
\text { Rats: } \downarrow \text { TL }(-31 \%) \text {, and } \uparrow \mathrm{TC}(+2 \%, \text { NS) and FC }(+6 \%, \text { NS }) \\
\text { contents }\end{array}$ & (Onning and Asp, 1995) \\
\hline $\begin{array}{l}45 \\
46 \\
47 \\
48\end{array}$ & $\begin{array}{l}\text { Soy saponins } \\
\text { Changkil saponins } \\
\quad \text { (from root of } \\
\text { Platycodon }\end{array}$ & $\begin{array}{l}\text { HepG2 cells } \\
\text { Mice fed saponins for } 7 \text { days before ethanol } \\
\text { administration }(5 \mathrm{~g} / \mathrm{kg} \text { b.w. }) \text { for around } 36 \text { hours }\end{array}$ & $\begin{array}{l}10 \mathrm{ng} / \mathrm{L} \\
0.5,1 \text { or } 2 \mathrm{mg} / \mathrm{kg} \\
\text { b.w. }\end{array}$ & $\begin{array}{l}24 \text { hours } \\
7 \text { days }\end{array}$ & $\begin{array}{l}\uparrow \operatorname{PPAR} \alpha(z+60 \%) \text { and PPAR } \gamma(z+80 \%) \\
\downarrow \text { dose-dependently TG content }(-7 \% \text {, NS, }-22 \text { and }-36 \%) \\
\text { Histopathological observations: } \downarrow \text { steatosis score }(-49 \%)\end{array}$ & $\begin{array}{l}\text { (Ricketts et al,, 2005) } \\
\text { (Khanal et al., 2009b) }\end{array}$ \\
\hline $\begin{array}{l}49 \\
50 \\
51 \\
52\end{array}$ & $\begin{array}{l}\text { grandiflorum) } \\
\text { Changkil saponins } \\
\text { (from root of } \\
\text { Platycodon } \\
\text { grandiflorum) }\end{array}$ & $\begin{array}{l}\text { Rats chronically fed with ethanol (enteral } \\
\text { feeding) for } 4 \text { weeks }\end{array}$ & $\begin{array}{l}0.5,1 \text { or } 2 \mathrm{mg} / \mathrm{kg} \\
\text { b.w. }\end{array}$ & Last 2 weeks & $\begin{array}{l}\text { Histologic observations: } \downarrow \text { fat deposition and faint micro- and } \\
\text { macrovesicular fat droplets } \\
\downarrow \text { TL content (resp. } \approx-15 \% \text {, NS, }-32 \text { and }-45 \% \text { ) } \\
\text { p phosphorylated-AMPK level (resp. }+16 \% \text {, NS, }+59 \text { and }+93 \% \text { ) }\end{array}$ & (Khanal et al., 2009a) \\
\hline
\end{tabular}




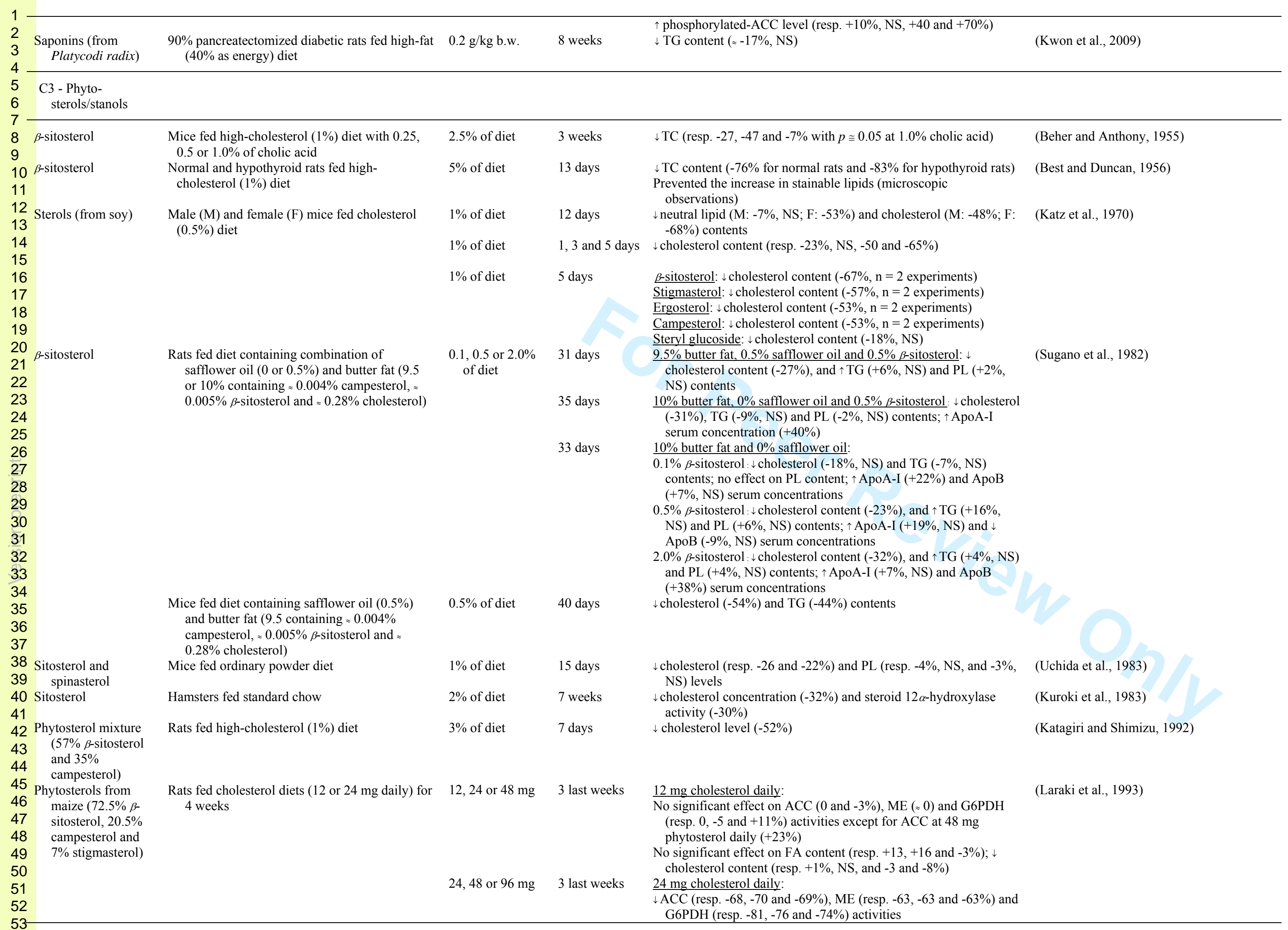




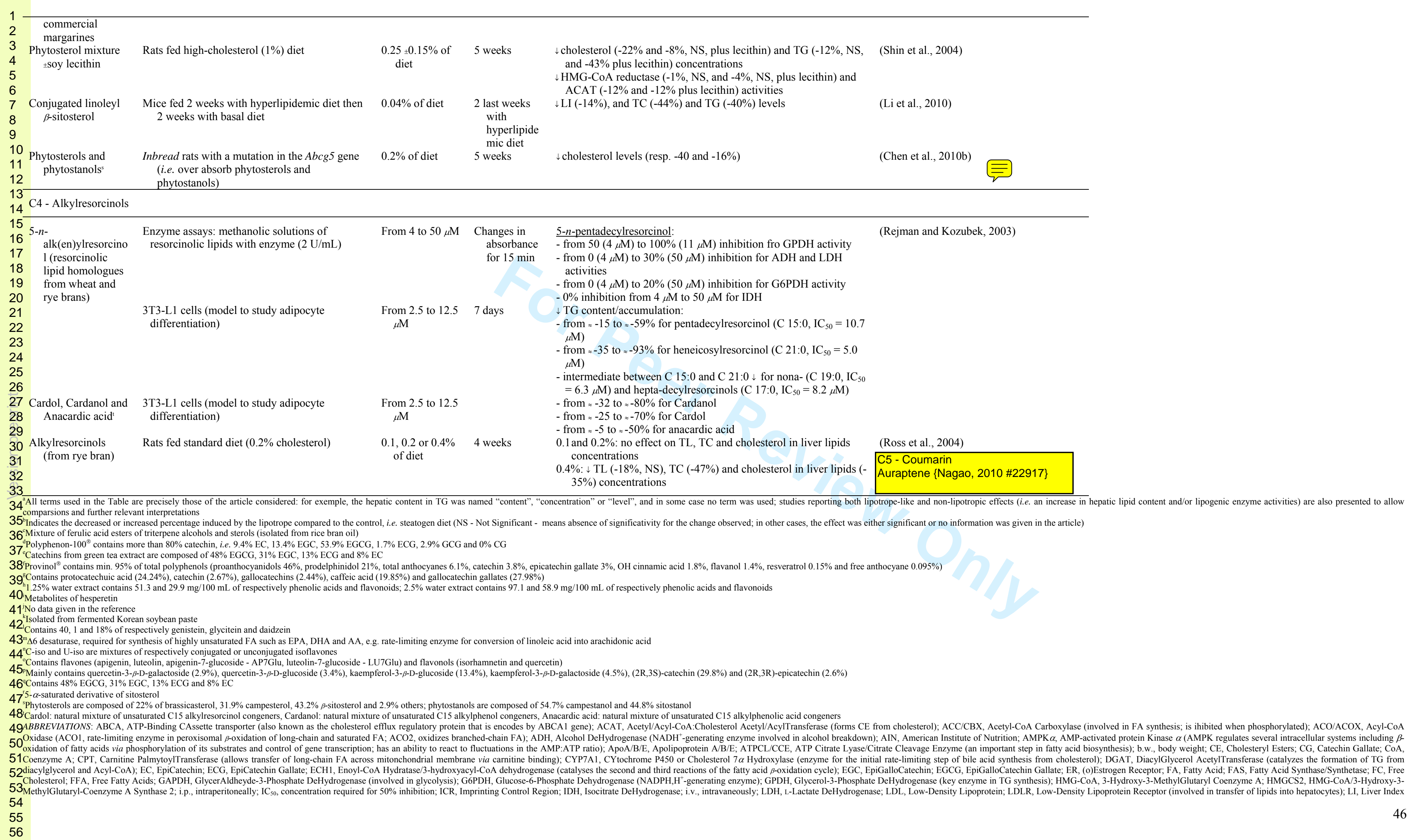




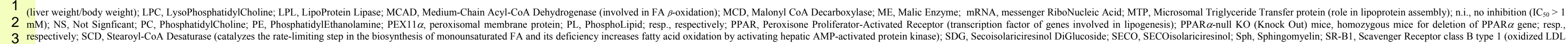

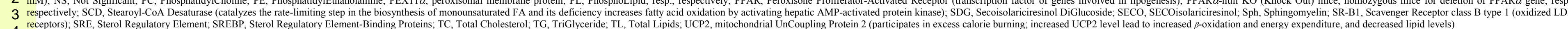


1 Supplemental Table 5 In vivo and in vitro studies reporting effects on hepatic lipid metabolism following supplementation of plant extracts or plant-based foods

\begin{tabular}{|c|c|c|c|c|c|}
\hline & $\begin{array}{l}\text { Plant extract or plant- } \\
\text { based foods }\end{array}$ & In vivo or in vitro models & $\begin{array}{l}\text { Supplemented } \\
\text { daily dose }\end{array}$ & $\begin{array}{l}\text { Duration of } \\
\text { lipotrope } \\
\text { exposition }\end{array}$ & Hepatic effect(s) \\
\hline & $\begin{array}{l}\text { Corn oil vs } \\
\text { hydrogenated } \\
\text { coconut oil } \\
\text { (control) }\end{array}$ & $\begin{array}{l}\text { Healthy male subjects fed ad libitum institutional } \\
\text { American type diet }\end{array}$ & $\begin{array}{l}\text { One ounce (24- } \\
\quad 33 \mathrm{~g})\end{array}$ & 1 month & $\begin{array}{l}\text { Corn oil: } \downarrow \text { liver cholesterol }(-25 \%)^{b} \text { upon } 1 \text { month } \\
\text { Hydrogenated coconut oil: } \\
\text { montiver cholesterol }(+9 \% \text {, NS) upon } 1\end{array}$ \\
\hline & $\begin{array}{l}\text { Cottonseer } v \text { vs } \\
\text { coconut oils }\end{array}$ & Rats fed $10 \%$-fat diet $\pm 1 \%$ cholesterol & $10 \%$ of diet & 7 weeks & $\begin{array}{l}\text { Males: adding cholesterol } \uparrow \text { TL }(+475 \text { vs }+110 \%)^{2} \text {, TC }(+1916 \text { vs } \\
+600 \%), \text { FC }(+95 \text { vs }+27 \%) \text { and PL }(+227 v s-3 \%) \text { contents } \\
\text { Females: adding cholesterol } \uparrow \text { TL }(+218 \text { vs }+75 \%) \text {, TC }(+2436 \text { vs }\end{array}$ \\
\hline $\begin{array}{l}13 \\
14 \\
15 \\
16 \\
17\end{array}$ & $\begin{array}{l}\text { Arachis oil, } 3 \\
\text { margarines } \\
\text { butter }\end{array}$ & Rabbits fed $20 \%$-fat diet (no control group) & $20 \%$ of diet & 42 weeks & 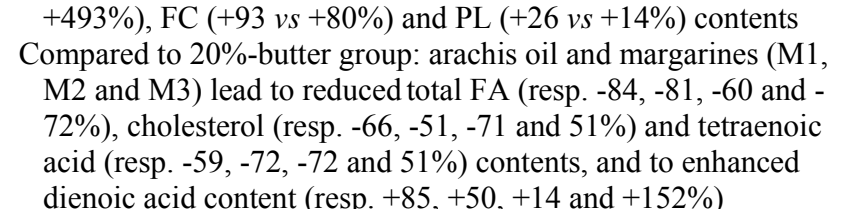 \\
\hline & $\begin{array}{l}\text { Whole wheat breads } \\
\text { or rye breads }\end{array}$ & Rats fed white breads & $-^{d}$ & & $\begin{array}{l}\text { Whole wheat breads: } \downarrow T G \text { (NS) and cholesterol levels } \\
\text { Rye breads: } \downarrow \text { TG level (NS) }\end{array}$ \\
\hline & Safflower oil & $\begin{array}{l}\text { Rats fed fat-free and high-fructose/glucose } \\
\text { (72\%) diet for } 7 \text { days then supplemented with } \\
\text { PUAA, injected with }{ }^{3} \mathrm{H}_{2} \mathrm{O} \text { and killed } 20 \mathrm{~min} \\
\text { after injection }\end{array}$ & $\begin{array}{l}5 \% \text { or } 10 \% \text { of } \\
\text { diet }\end{array}$ & 3 or 4 days & $\begin{array}{l}\text { Fructose: } \downarrow \text { FAS }(-50-64 \% \text { at } 5 \% \text { fat level), ACC }(-57 \% \text { at } 10 \% \text { fat } \\
\text { level), glucokinase }(-19 \% \text {, NS at } 10 \% \text { fat level) and } \\
\text { phosphofructokinase (-10\%, NS at } 5 \% \text { fat level) activities; } \downarrow \text { FA } \\
\text { synthesis }(-32 \% \text { at } 5 \% \text { fat level and }-76 \% \text { at } 10 \% \text { level) } \\
\text { Glucose: } \downarrow \text { FAS }(-71 \% \text { at } 5 \% \text { fat level) and phosphofiuctokinase (- } \\
7 \% \text {, NS at } 5 \% \text { fat level) activities; } \downarrow \text { FA synthesis }(-53 \% \text { at } 5 \% \\
\text { fat level) }\end{array}$ \\
\hline & 等 & $\begin{array}{l}\text { Rats fed high-cholesterol }(1 \%+0.5 \% \text { cholic acid }) \\
\text { diet }\end{array}$ & $10 \%$ of diet & 8 weeks & $\downarrow$ TC $(-22 \%, N S)$ and TG $(-32 \%, N S)$ contents \\
\hline
\end{tabular}
References $\begin{array}{cc}\text { Rats trained } 10 \text { days with high-glucose }(58.43 \%) & 20 \% \text { digestible } \\ \text { and fat-free diet, then supplemented } 7 \text { days } & \text { energy of diet }\end{array}$ with PUFA-rich oils or tripalmitin (control) Rats trained 10 days with high-glucose $(58.43 \%) \quad 20 \%$ digestible $\quad 7$ days and fat-free diet, then supplemented 7 days energy of diet Male hamsters fed $0.3 \%$-cholesterol diets (all Resp. $9,35,35+21$ days contains $10 \%$ fiber and $9 \%$ fat)

8.9, and 43.7, $32.8,21.8$ an $10.9 \%$ Hypercholesterolemic hamsters (control diet
contains $0.3 \%$ cholesterol, $10 \%$ cellulose and Resp. 50.2, 41.3- 21 days Resp. $50.2,41.3-$
$41.5,7.9-9.0$
0.9 and $0.2 \%$ fat and $3 \%$ nitrogen of diet

(Frantz and Carey, 1961)

(Okey et al., 1961)

(Krogh et al., 1961)

(Yacowitz et al., 1976)

(Toussant et al., 1981)

FAS mRNA abundance ( $z-69 \%$ for $n-6$-rich safflower oil and $z-$ $87 \%$ for $n-3$-rich menhaden fish oil)

$\downarrow$ transcription rate of FAS $(-94 \%)$ and $\mathrm{S}_{14}$ protein (putative (IT) c cholesterol content (resp. $-5 \%$, NS, $-18,-15,-24,-8 \%$, NS, $-12 \%$,
NS, and $-3 \%$ ) TG content (resp. $\approx 0,+17 \%$, NS, $+13 \%$, NS, $+11 \%$, NS, $+13 \%$, NS, $+14 \%, \mathrm{NS}$, and $z 0$ )

bran: resp. -37 and $-33 \%$ -defatted bran: $-12 \%$, NS, and $-26 \%$, NS defatted bran +rice bran oil gum: resp. $-1 \%$, NS, and $-24 \%$

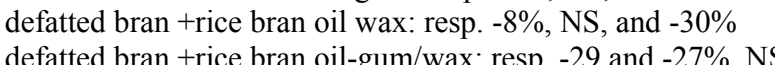

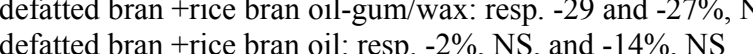
$\downarrow$ cholesterol pool (-23\% ${ }^{3}$ for oat bran vs wheat bran; - $13 \%$, NS, for (Jackson et al., 1994) barley $v s$ malted barley) lignin) of diet Rats fed AIN 76-based an
$+0.1 \%$ cholic acid) diet esterol pool $(+15 \%$, NS,
barley vs wheat bran)

(2) rate of [1- $C$ ]acetate incorporation into cholesterol at $0.1(-72 \%)$ Hepatocytes isolated from rat liver and incubated $0.01,0.05,0.1, \quad 4$ hou 02 and 0.4 0.2 and 0.4
$\mathrm{mM}$ with $0.5 \mathrm{mM}\left[1{ }^{-14} \mathrm{C}\right]$ acetate $(-87 \%) \mathrm{mM}$; no significant changes at other Rats and gerbils fed high-fat $(40 \%)$ and $6.5 \% \quad 6.5 \%$ of diet Gerbils: $\downarrow$ TL (resp. $-2 \%$, NS, and $-1 \%$, NS), TC (-38 and $-34 \%$ )
- 


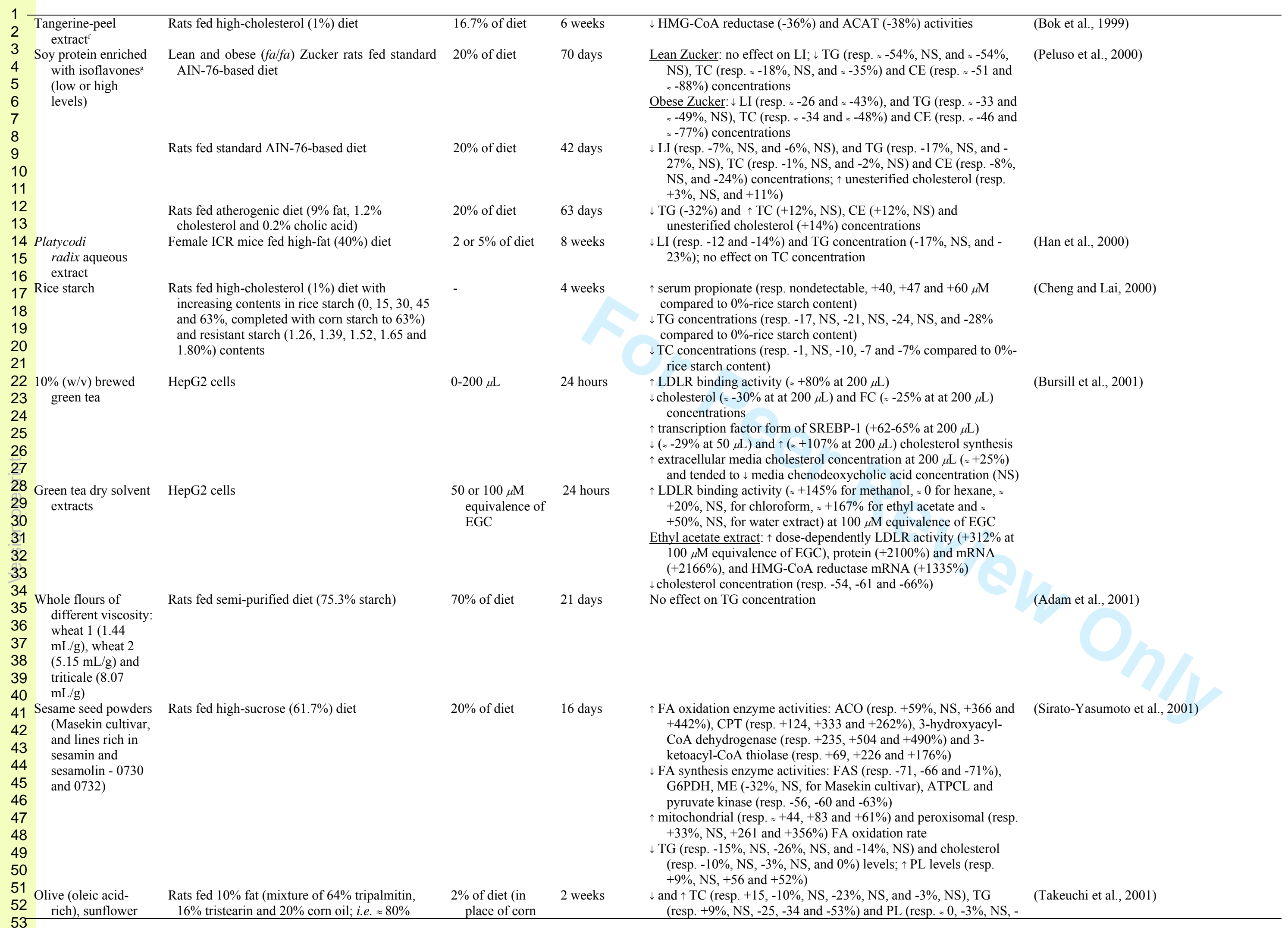




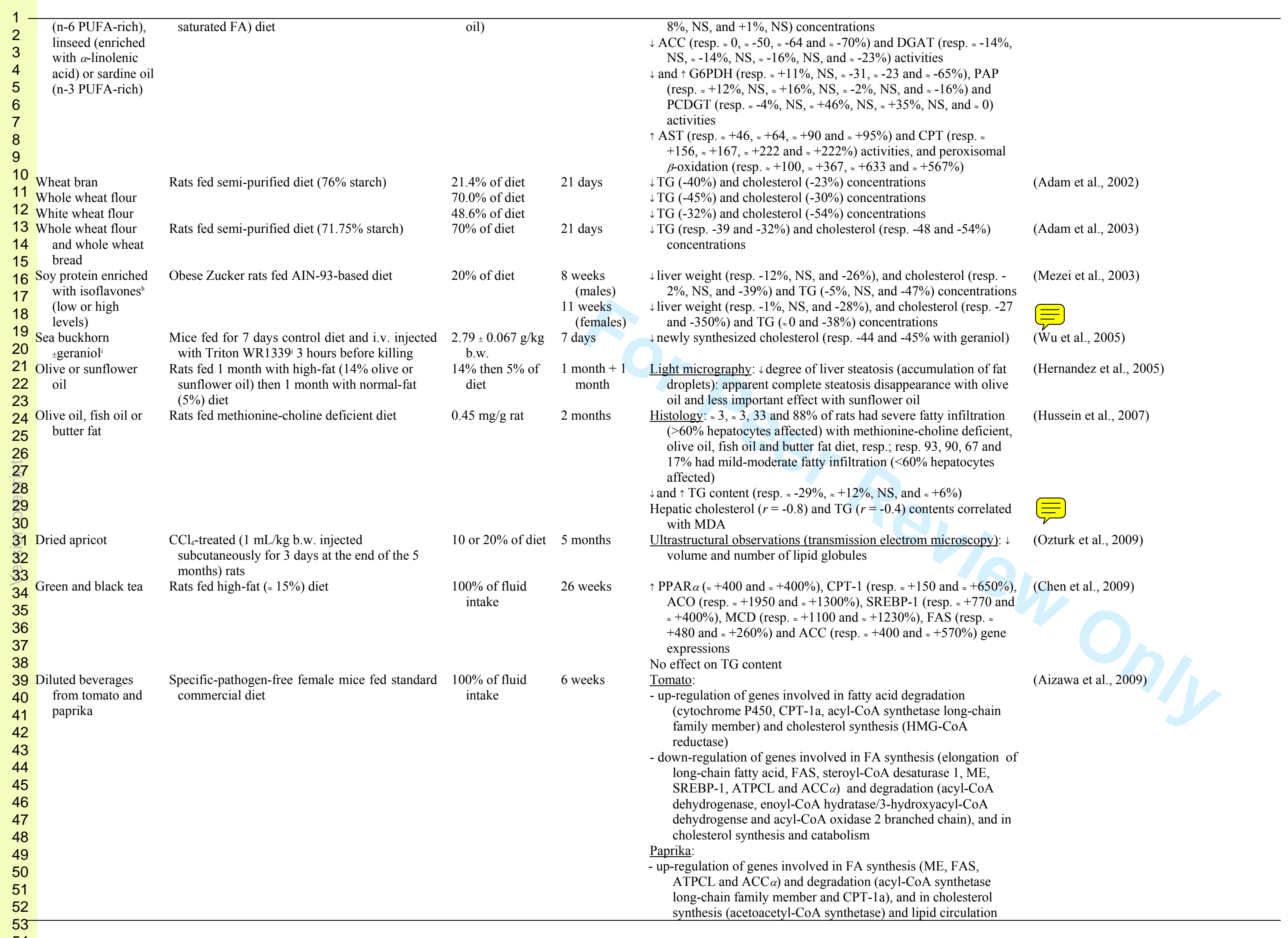




\begin{tabular}{|c|c|c|c|c|c|c|}
\hline & & & $2 \mathrm{~g} / \mathrm{kg}$ b.w. & 8 weeks & $\begin{array}{l}\text { Post-administration ( } 2 \text { weeks without alcohol) after } 6 \text { weeks } \\
\text { alcohol: } \downarrow \text { cholesterol (resp. }-15 \text { and }-35 \% \text { ) and TG (resp. }-30 \text { and } \\
-38 \% \text { ) contents } \\
\downarrow \text { TG content }(z-44 \%)\end{array}$ & (Kwon et al., 2009) \\
\hline & extract & ( $40 \%$ as energy) diet & grkg u.w. & oweens & & \\
\hline & $\begin{array}{l}\text { Codonopsis } \\
\text { lanceolata root }\end{array}$ & Rats fed liquid ethanol (36\% of energy) diet & $\begin{array}{l}0.5 \% \text { of liquid } \\
\text { diet }\end{array}$ & 8 weeks & $\begin{array}{l}\downarrow \mathrm{TG}(\approx-33 \%) \text { and cholesterol }(\approx-36 \%) \text { levels } \\
\text { Liver histology: enlargement of the hepatocytes and increase in the }\end{array}$ & (Cho et al, 2009a) \\
\hline & water extract & & & & number of lipid droplets were normalized & \\
\hline & & & & & $\downarrow \operatorname{tNF} \alpha(z-37 \%), \operatorname{LXR} \alpha(z-17 \%)$, SREBP-1c $(z-21 \%)$, HMG-CoA & \\
\hline & & & & & $\uparrow \operatorname{AMPK} \alpha(z+6 \%, \mathrm{NS}), \mathrm{ACC}(z+48 \%), \mathrm{FAS}(+29 \%)$ and SCD1 $(z$ & \\
\hline & & & & & $\begin{array}{l}+10 \%, \text { NS) mRNA levels } \\
\text { a }\end{array}$ & \\
\hline & & & & & 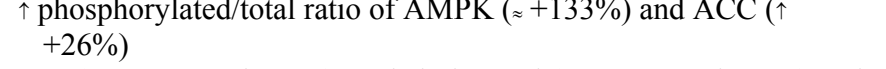 & \\
\hline & $\begin{array}{l}4 \text { Commercial low- } \\
5 \text { trans fat or } \mathrm{n}-3 \text { - }\end{array}$ & $\begin{array}{l}\text { Apo } \mathrm{E}^{-m} \text { mice fed a 10\%-fat (commercial } \\
\text { shortening, } 53.4 \% \text { trans FA) diet }\end{array}$ & $10 \%$ of diet & 12 weeks & $\begin{array}{l}\downarrow \mathrm{LI} \text { (resp. }-34 \text { and }-44 \% \text { ), and cholesterol (resp. }-31 \text { and }-41 \% \text { ) and } \\
\text { TG (resp }-22 \text { and }-16 \% \text { levers }\end{array}$ & (Cho et al., 2009b) \\
\hline & 6 rich/low-trans & & & & $\downarrow$ HMG-CoA reductase (resp. $12 \%$, NS, and - $51 \%$ ), ACAT (resp. - & \\
\hline & $7 \begin{array}{c}\text { structured fat } \\
\text { (synthesizeed }\end{array}$ & & & & 6\%, NS, and - $18 \%$ ), G6PDH (resp. -52 and -66\%), ME (resp. - 25 & \\
\hline & 8 from flaxseed oil, & & & & 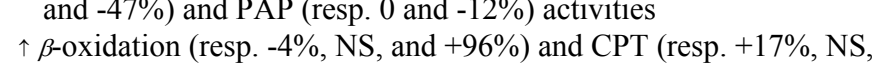 & \\
\hline & anhydrous & & & & and + $88 \%$ ) activity & \\
\hline & $\begin{array}{l}1 \text { butterfat and palm } \\
\text { stearin) }\end{array}$ & & & & $\begin{array}{l}\text { Hepatic tissue morphology: low-trans structured fat importantly } \\
\text { accumulation of hepatic lipid droplets }\end{array}$ & \\
\hline & $\begin{array}{l}2 \text { Fermented ginseng } \\
3 \text { radix ethanol }\end{array}$ & HepG2 cells & $500 \mu \mathrm{g} / \mathrm{mL}$ & $\begin{array}{l}\text { From } 1 \text { to } 24 \\
\text { bours }\end{array}$ & $\uparrow$ phosphorylation of AMPK (max.: $: 2.7$-fold at 12 hours) and ACC & (Kim et al., 2009) \\
\hline & 4 extract & & & & $\downarrow$ time-dependently SREBP1c, SCD1 and FAS gene expression; $\uparrow$ & \\
\hline & & & $\begin{array}{l}100,250 \text { or } 500 \\
\mu \mathrm{g} / \mathrm{mL}\end{array}$ & 24 hours & 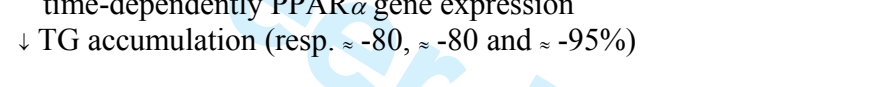 & \\
\hline & & $d b / d b$ mice fed standard chow diet & $\begin{array}{l}100 \text { or } 200 \\
\mathrm{mg} / \mathrm{kg} \text { b.w. }\end{array}$ & 10 weeks & $\begin{array}{l}\uparrow \text { gene expression of pAMPK and pACC ( } \uparrow \text { phosphorylation of } \\
\text { AMPK and ACC), and of CD36 and PPAR } \alpha ; \text { gene expression of } \\
\text { SREBP } 1 \text { a, SCD1 and FAS }\end{array}$ & \\
\hline & $\begin{array}{l}0 \text { Garlic }+ \text { medicinal } \\
1 \\
2\end{array}$ plant extracts & $\begin{array}{l}\text { Rats fed ethanol }(10 \mathrm{~mL} \text { of } 20 \% \text { ethanol } / \mathrm{kg} \\
\text { b.w./day) diet }\end{array}$ & $\begin{array}{l}(0.5+1 . \%) \text { or } \\
\quad(1.0+1.0) \% \\
\text { of diet }\end{array}$ & 4 weeks & $\begin{array}{l}\downarrow \mathrm{TL} \text { (resp. - } 14 \text { and }-28 \% \text { ), TC (resp. }-17 \text { and }-23 \% \text { ) and TG (resp. - } \\
9 \% \text {, NS, and }-30 \% \text { ) levels }\end{array}$ & (Soo-Jung et al., 2009) \\
\hline & $\begin{array}{ll}3 & \text { Refined rice bran oil, } \\
4 & \text { alone or blended } \\
5 & \text { with refined } \\
6 & \text { linseed oil (3:2, } \\
7 & \text { w/w) or cod liver } \\
& \text { oil (1:1) }\end{array}$ & Rats fed $10 \%$-fat (refined groundnut oil) diet & $10 \%$ of diet & 60 days & $\begin{array}{l}\downarrow \text { TC (resp. }-14,-27 \text { and }-37 \% \text { ), PL (resp. }-13,-35 \text { and }-40 \% \text { ) and } \\
\text { TG (resp. }-9,-19 \text { and }-26 \% \text { ) contents }\end{array}$ & (Chopra and Sambaiah, 2009) \\
\hline & 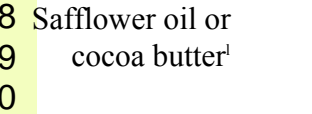 & Rats fed standard chow ( $4 \%$ fat) & $\begin{array}{l}29.5 \text { or } 29 \% \text { of } \\
\text { diet' }^{\prime}\end{array}$ & 3 days & $\begin{array}{l}\uparrow \text { TG (resp. } \approx+59 \text { and } z+60 \%) \text { and cholesterol (resp. } \approx+21 \%, \text { NS, } \\
\text { and } \approx+71 \% \text { levels } \\
\downarrow \text { SCD- } 1 \text { mRNA expression (resp. } z-62 \text { and } z-81 \%)\end{array}$ & (Gustavsson et al., 2009) \\
\hline & & & & & $\begin{array}{l}\downarrow \downarrow \text { SCD-1 mRNA expression (resp. } \approx-62 \text { and } \approx-81 \% \text { ) } \\
\text { Cocoa butter: }\end{array}$ & \\
\hline & 2 & & & & $\uparrow$ ATPCL (4.00-fold), ME 1 (1.81-fold), pyruvate kinase (1.59- & \\
\hline & & & & & 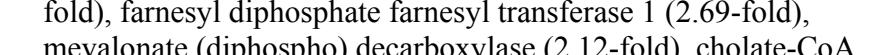 & \\
\hline & & & & & ligase (2.01-fold), HMG-CoA synthase (1.89-fold), squalene & \\
\hline & & & & & epoxidase (1.87-fold), 7 -dehydrocholesterol reductase (1.72-fold), & \\
\hline & & & & & lanosterol synthase (1.72-fold), farnesyl diphosphate synthase & \\
\hline & & & & & (1.66-fold), bile acid-CoA ligase (1.63-fold), ACAT 2 (2.00- & \\
\hline & & & & & fold), ACAT 1 (1.63-fold), FAD 1 (1.71-fold), FAD 2 (1.57-fold) & \\
\hline & & & & & and FAD 3 (1.69-fold) mRNA expression; $\downarrow$ GPDH 1 (0.63-fold), & \\
\hline & & & & & ABC subfamily A (0.46-fold), mitochondrial acyl-CoA & \\
\hline & & & & & 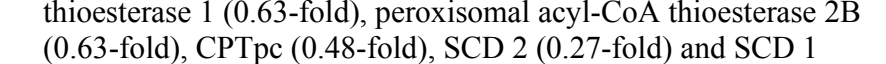 & \\
\hline & 2 & & & & (0.22-fold) mRNA expression & \\
\hline
\end{tabular}




\section{Page 221 of 267}

Critical Reviews in Food Science and Nutrition

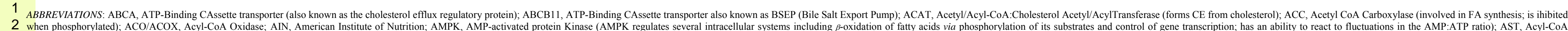

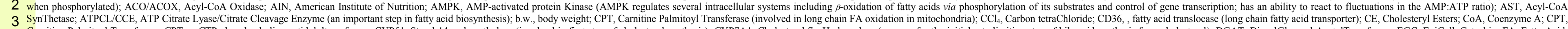

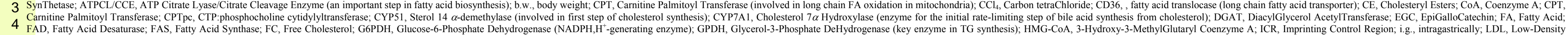

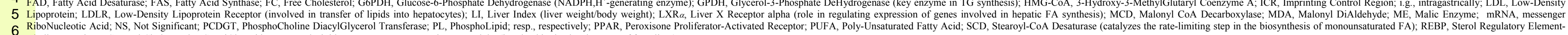

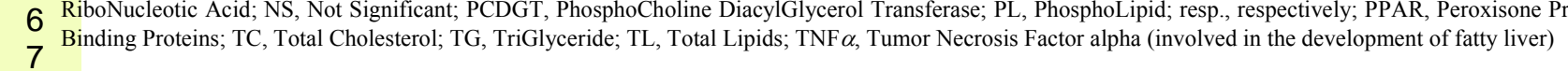

7
8
9
10
11
12
13
14
15
16
17
18
19
19
21
22
22
24
24
26
27
28
29
30
31
32
33
34
35
36
37
38
39
40
41
42
43
44
45
46
47
48
49
50
51
52
53
54
55
56
57
58
59
60 


\section{REFERENCES}

Abdelmalek, M. F., Angulo, P., Jorgensen, R. A., Sylvestre, P. B. and Lindor, K. D. (2001). Betaine, a promising new agent for patients with nonalcoholic steatohepatitis: results of a pilot study. American Journal of Gastroenterology 96: 2711-2717.

Adam, A., Levrat-Verny, M. A., Lopez, H. W., Leuillet, M., Demigne, C. and Remesy, C. (2001). Whole wheat and triticale flours with differing viscosities stimulate cecal fermentations and lower plasma and hepatic lipids in rats. Journal of Nutrition 131: 1770-1776.

Adam, A., Lopez, H. W., Leuillet, M., Demigne, C. and Remesy, C. (2003). Whole wheat flour exerts cholesterol-lowering in rats in its native form and after use in bread-making. Food Chemistry 80: 337-344.

Adam, A., Lopez, H. W., Tressol, J. C., Leuillet, M., Demigne, C. and Remesy, C. (2002). Impact of whole wheat flour and its milling fractions on the cecal fermentations and the plasma and liver lipids in rats. Journal of Agricultural and Food Chemistry 50: 6557-6562.

Aghelli, N., Kabir, M., Berni-Canani, S., Petitjean, E., Boussairi, A., Luo, J., Bornet, F., Slama, G. and Rizkalla, S. W. (1998). Plasma lipids and fatty acid synthase activity are regulated by shortchain fructo-oligosaccharides in sucrose-fed insulin-resistant rats. Journal of Nutrition 128: 12831288.

Aiyar, A. S., Sulebele, G. A., Rege, D. V. and Sreenivasan, A. (1959). Pantothenic acid deficiency and ubiquinone levels in rat liver mitochondria. Nature 184: 1867-1868.

Aizawa, K., Matsumoto, T., Inakuma, T., Ishijima, T., Nakai, Y., Abe, K. and Amano, F. (2009). Administration of tomato and paprika beverages modifies hepatic glucose and lipid metabolism in mice: a DNA microarray analysis. Journal of Agricultural and Food Chemistry 57: 10964-10971.

Allmann, D. W. and Gibson, D. M. (1965). Fatty acid synthesis during early linoleic acid deficiency in the mouse. Journal of Lipid Research 6: 51-62. 
Alshatwi, A. A., Al Obaaid, M. A., Al Sedairy, S. A., Al-Assaf, A. H., Zhang, J. J. and Lei, K. Y.

(2010). Tomato powder is more protective than lycopene supplement against lipid peroxidation in rats. Nutrition Research 30: 66-73.

Alwayn, I. P. J., Andersson, C., Zauscher, B., Gura, K., Nosé, V. n. and Puder, M. (2005a). Omega3 fatty acids improve hepatic steatosis in a murine model: potential implications for the marginal steatotic liver donor. Transplantation Proceedings 79: 606-608.

Alwayn, I. P. J., Gura, K., Nosé, V., Zaosche, B., Javid, P., Garza, J., Verbesey, J., Voss, S., Ollero, M., Andersson, C., Bistrian, B., Folkman, J. and Puder, M. (2005b). Omega-3 Fatty Acid Supplementation Prevents Hepatic Steatosis in a Murine Model of Nonalcoholic Fatty Liver Disease. Pediatric Research 57: 445-452.

Andersen, D. B. and Holub, B. J. (1980a). Myo-inositol-responsive liver lipid accumulation in the rat. Journal of Nutrition 110: 488-495.

Andersen, D. B. and Holub, B. J. (1980b). The relative response of hepatic lipids in the rat to graded levels of dietary myo-inositol and other lipotropes. Journal of Nutrition 110: 496-504.

Andrieux-Domont, C. and Le van, H. (1970). Influence of magnesium on enzymatic synthesis of coenzyme A. Comptes Rendus des Séances de la Société de Biologie et de ses Filiales 164: 292 296.

Arachchige, P. G., Takahashi, Y. and Ide, T. (2006). Dietary sesamin and docosahexaenoic and eicosapentaenoic acids synergistically increase the gene expression of enzymes involved in hepatic peroxisomal fatty acid oxidation in rats. Metabolism-Clinical and Experimental 55: 381390.

Arhan, M., Ozturk, H. S., Turhan, N., Aytac, B., Guven, M. C., Olcay, E. and Durak, I. (2009). Hepatic oxidant/antioxidant status in cholesterol-fed rabbits: effects of garlic extract. Hepatology Research 39: 70-77. 
Arjmandi, B. H., Ahn, J., Nathani, S. and Reeves, R. D. (1992a). Dietary soluble fiber and cholesterol affect serum-cholesterol concentration, hepatic portal venous short-chain fatty-acid concentrations and fecal sterol excretion in rats. Journal of Nutrition 122: 246-253.

Arjmandi, B. H., Craig, J., Nathani, S. and Reeves, R. D. (1992b). Soluble dietary fiber and cholesterol influence invivo hepatic and intestinal cholesterol-biosynthesis in rats. Journal of Nutrition 122: 1559-1565.

Ashakumary, L., Rouyer, I., Takahashi, Y., Ide, T., Fukuda, N., Aoyama, T., Hashimoto, T., Mizugaki, M. and Sugano, M. (1999). Sesamin, a sesame lignan, is a potent inducer of hepatic fatty acid oxidation in the rat. Metabolism 48: 1303-1313.

Babenko, N. A. and Shakhova, E. G. (2006). Effects of Chamomilla recutita flavonoids on agerelated liver sphingolipid turnover in rats. Experimental Gerontology 41: 32-39.

Badmaev, V., Majeed, M. and Conte, A. A. (2002). Open field, physician controlled clinical evaluation of a botanical weight loss formula based on Garcinia cambogia derived (hydroxycitric acid. NutraCos 1: 10-14.

Baker, H., Luisada-Opper, A., Sorrell, M. F., Thomson, A. D. and Frank, O. (1973). Inhibition by nicotinic acid of hepatic steatosis and alcohol dehydrogenase in ethanol-treated rats. Experimental and Molecular Pathology 19: 106-112.

Balkan, J., Oztezcan, S., Kucuk, M., Cevikbas, U., Kocak-Toker, N. and Uysal, M. (2004). The effect of betaine treatment on triglyceride levels and oxidative stress in the liver of ethanol-treated guinea pigs. Experimental and Toxicologic Pathology 55: 505-509.

Ball, C. R. (1964). Actions of betaine, carnitine and choline on pattern of hepatic liposis in mice fed high-fat low-protein diet. Anatomical Record 149: 677-689.

Barak, A. J., Beckenhauer, H. C., Badakhsh, S. and Tuma, D. J. (1997). The effect of betaine in reversing alcoholic steatosis. Alcoholism: Clinical and Experimental Research 21: 1100-1102.

Barak, A. J., Beckenhauer, H. C. and Tuma, D. J. (1996). Betaine effects on hepatic methionine metabolism elicited by short-term ethanol feeding. Alcohol 13: 483-486.

Comment citUred httph:P/mc.manuscriptcentral.com/bfsn Email: fergc@foodsci.umass.edu Fardet, A., Chardigny, J.-M. (2013). Plant cinder.

Nutrition: A Survey of In Vivo Studies. Critical Reviews in Food Science
535-590. DOI : 10.1080/10408398.2010.549596 
Beach, D. C. and Flick, P. K. (1982). Early effect of myoinositol deficiency on fatty-acid synthetic enzymes of rat-liver. Biochimica et Biophysica Acta 711: 452-459.

Beher, W. T. and Anthony, W. L. (1955). Effects of $\beta$-sitosterol and ferric chloride on accumulation of cholesterol in mouse liver. Proceedings of the Society for Experimental Biology and Medicine 90: $223-225$.

Benkhalti, D., Prost, J., Paz, E., Perez-Jimenez, F., El Modafar, C. and El Boustani, E. (2002). Effects of feeding virgin olive oil or their polyphenols on lipid of rat liver. Nutrition Research 22 1067-1075.

Berkhout, T. A., Havekes, L. M., Pearce, N. J. and Groot, P. H. E. (1990). The effect of (-)hydroxycitrate on the activity of the low-density-lipoprotein receptor and 3-hydroxy-3methylglutaryl-CoA reductase levels in the human hepatoma-cell line Hep G2. Biochemical Journal 272: 181-186.

Best, C. H. (1934). The role of the liver in the metabolism of carbohydrate and fat. III - The deposition of liver fat. The Lancet 223: 1274-1277.

Best, C. H. and Huntsman, M. E. (1932). The effects of the components of lecithine upon deposition of fat in the liver. Journal of Physiology 75: 405-412.

Best, C. H. and Huntsman, M. E. (1935). The effect of choline on the liver fat of rats in various states of nutrition. Journal of Physiology 83: 255-274.

Best, C. H., Lucas, C. C., Ridout, J. H. and Patterson, J. M. (1950). Dose-response curves in the estimation of potency of lipotropic agents. Journal of Biological Chemistry 186: 317-329.

Best, C. H. and Ridout, J. H. (1940). The lipotropic action of methionine. Journal of Physiology 97: 489-494.

Best, M. M. and Duncan, C. H. (1956). Effects of sitosterol on the cholesterol concentration in serum and liver in hypothyroidism. Circulation 14: 344-348.

Beveridge, J. M. R., Lucas, C. C. and O'Grady, M. K. (1945). The effect of dietary proteins and amino acids on liver fat. Journal of Biological Chemistry 160: 505-518.

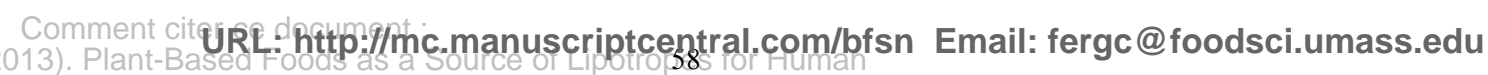
Fardet, A., Chardigny, J.-M. (2013). Plant-Based Foods as a Soulce or Lnpotro 58 s iof

Nutrition: A Survey of In Vivo Studies. Critical Reviews in Food Science
535-590. DOI : 10.1080/10408398.2010.549596 
Blake, W. L. and Clarke, S. D. (1990). Suppression of rat hepatic fatty acid synthase and s14 gene transcription by dietary polyunsaturated fat. Journal of Nutrition 120: 1727-1729.

Bok, S.-H., Lee, S.-H., Park, Y.-B., Bae, K.-H., Son, K.-H., Jeong, T.-S. and Choi, M.-S. (1999). Plasma and hepatic cholesterol and hepatic activities of 3-hydroxy-3-methyl-glutaryl-CoA reductase and acyl CoA: cholesterol transferase are lower in rats fed citrus peel extract or a mixture of citrus bioflavonoids. Journal of Nutrition 129: 1182-1185.

Borgschulte, G., Kathirvel, E., Herrera, M., French, S. W., Morgan, T. R., Morgan, K. and Bottiglieri, T. (2008). Betaine treatment reverses insulin resistance and fatty liver disease without reducing oxidative stress or endoplasmic reticulum stress in an animal model of NAFLD. Gastroenterology 134: A414-A415.

Bortolotti, M., Kreis, R., Debard, C., Cariou, B., Faeh, D., Chetiveaux, M., Ith, M., Vermathen, P., Stefanoni, N., Le, K.-A., Schneiter, P., Krempf, M., Vidal, H., Boesch, C. and Tappy, L. (2009). High protein intake reduces intrahepatocellular lipid deposition in humans. American Journal of Clinical Nutrition 90: 1002-1010.

Bose, M., Lambert, J. D., Ju, J., Reuhl, K. R., Shapses, S. A. and Yang, C. S. (2008). The major green tea polyphenol, (-)-epigallocatechin-3-gallate, inhibits obesity, metabolic syndrome, and fatty liver disease in high-fat-fed mice. Journal of Nutrition 138: 1677-1683.

Bowyer, B. A., Miles, J. M., Haymond, M. W. and Fleming, C. R. (1988). L-carnitine therapy in home parenteral-nutrition patients with abnormal liver tests and low plasma carnitine concentrations. Gastroenterology 94: 434-438.

Brandt, K., Langhans, W., Geary, N. and Leonhardt, M. (2006). Beneficial and deleterious effects of hydroxycitrate in rats fed a high-fructose diet. Nutrition 22: 905-912.

Bruno, R. S., Dugan, C. E., Smyth, J. A., DiNatale, D. A. and Koo, S. I. (2008). Green tea extract protects leptin-deficient, spontaneously obese mice from hepatic steatosis and injury. Journal of Nutrition 138: 323-331. 
Buchman, A. L., Ament, M. E., Sohel, M., Dubin, M., Jenden, D. J., Roch, M., Pownall, H., Farley,

W., Awal, M. and Ahn, C. (2001). Choline deficiency causes reversible hepatic abnormalities in patients receiving parenteral nutrition: proof of a human choline requirement: A placebocontrolled trial. Journal of Parenteral and Enteral Nutrition 25: 260-268.

Buchman, A. L., Dubin, M. D., Moukarzel, A. A., Jenden, D. J., Roch, M., Rice, K. M., Gornbein, J. and Ament, M. E. (1995). Choline deficiency: a cause of hepatic steatosis during parenteral nutrition that can be reversed with intravenous choline supplementation. Hepatology 22: 13991403.

Bursill, C., Roach, P. D., Bottema, C. D. and Pal, S. (2001). Green tea upregulates the low-density lipoprotein receptor through the sterol-regulated element binding protein in HepG2 liver cells. Journal of Agricultural and Food Chemistry 49: 5639-5645

Bursill, C. A. and Roach, P. D. (2006). Modulation of cholesterol metabolism by the green tea polyphenol (-)-epigallocatechin gallate in cultured human liver (HepG2) cells. Journal of Agricultural and Food Chemistry 54: 1621-1626.

Burton, L. E. and Wells, W. W. (1977). Characterization of the lactation-dependent fatty liver in myo-inositol deficient rats. Journal of Nutrition 107: 1871-1883.

Busserolles, J., Gueux, E., Rock, E., Demigne, C., Mazur, A. and Rayssiguier, Y. (2003). Oligofructose protects against the hypertriglyceridemic and pro-oxidative effects of a high fructose diet in rats. Journal of Nutrition 133: 1903-1908.

Caballero, F., Fernandez, A., Fernandez-Checa, J. C. and Garcia-Ruiz, C. (2008). Methionine deficiency accounts for the liver damage observed in a nutritional model of nonalcoholic steatohepatitis. Journal of Hepatology 48: 917.

Capanni, M., Calella, F., Biagini, M. R., Genise, S., Raimondi, L., Bedogni, G., Svegliati-Baroni, G., Sofi, F., Milani, S., Abbate, R., Surrenti, C. and Casini, A. (2006). Prolonged n-3 polyunsaturated fatty acid supplementation ameliorates hepatic steatosis in patients with non- 
alcoholic fatty liver disease: a pilot study. Alimentary Pharmacology and Therapeutics 23: 1143 1151.

Carroll, C. and Williams, L. (1982). Choline deficiency in rats as influenced by dietary energysources. Nutrition Reports International 25: 773-782.

Casaschi, A., Rubio, B. K., Maiyoh, G. K. and Theriault, A. G. (2004). Inhibitory activity of diacylglycerol acyltransferase (DGAT) and microsomal triglyceride transfer protein (MTP) by the flavonoid, taxifolin, in HepG2 cells: potential role in the regulation of apolipoprotein B secretion. Atherosclerosis 176: 247-253

Catolla Cavalcanti, A. and Levis, F. (1950). Steatosis of the liver due to phosphorus. I. Lipotropic action of pantothenic acid and of its combination with meso-inositol. Archivio per le Scienze Mediche (Torino) 90: 529-541.

Causi, N., Romano, A. and Galfano, G. (1958). Coenzyme A in rat liver during administration of pantothenic acid and nicotinamide. Bollettino-Societa Italiana Biologia Sperimentale (Napoli) 34: 163-164.

Cha, J. Y., Cho, Y. S., Kim, I., Anno, T., Rahman, S. M. and Yanagita, T. (2001). Effect of hesperetin, a citrus flavonoid, on the liver triacylglycerol content and phosphatidate phosphohydrolase activity in orotic acid-fed rats. Plant Foods for Human Nutrition 56: 349-358.

Chahl, J. S. and Kratzing, C. C. (1966a). Environmental temperature and choline requirement in rats . 1. Choline deficiency in rats at various temperatures. Journal of Lipid Research 7: 17-21.

Chahl, J. S. and Kratzing, C. C. (1966b). Environmental Temperature and Choline Requirements in Rats . 2. Choline and Methionine Requirements for Lipotropic Activity. Journal of Lipid Research 7: 22-26

Chakrabarti, C. and Banerjee, B. (1969). Influence of riboflavin, choline, inositol and niacin on hepatic biosynthesis of cholesterol of albino rats kept on a high cholesterol diet. Journal of Vitaminology 15: 204-207. 
Chan, M. Y. and Heng, C. K. (2008). Sequential effects of a high-fiber diet with psyllium husks on the expression levels of hepatic genes and plasma lipids. Nutrition 24: 57-66.

Chan, P. T., Fong, W. P., Cheung, Y. L., Huang, Y., Ho, W. K. K. and Chen, Z.-Y. (1999). Jasmine green tea epicatechins are hypolipidemic in hamsters (Mesocricetus auratus) fed a high fat diet. Journal of Nutrition 129: 1094-1101.

Chan, T. Y. and Tang, P. L. (1995). Effect of melatonin on the maintenance of cholesterol homeostasis in the rat. Endocrine Research 21: 681-696.

Chen, N., Bezzina, R., Hinch, E., Lewandowski, P. A., Cameron-Smith, D., Mathai, M. L., Jois, M., Sinclair, A. J., Begg, D. P., Wark, J. D., Weisinger, H. S. and Weisinger, R. S. (2009). Green tea, black tea, and epigallocatechin modify body composition, improve glucose tolerance, and differentially alter metabolic gene expression in rats fed a high-fat diet. Nutrition Research 29: 784-793.

Chen, H., Liu, L.-j., Zhu, J.-j., Xu, B. and Li, R. (2010a). Effect of soybean oligosaccharides on blood lipid, glucose levels and antioxidant enzymes activity in high fat rats. Food Chemistry 119: 1633-1636.

Chen, Q., Gruber, H., Swist, E., Coville, K., Pakenham, C., Ratnayake, W. and Scoggan, K. (2010b). Dietary phytosterols and phytostanols decrease cholesterol levels but increase blood pressure in WKY inbred rats in the absence of salt-loading. Nutrition and Metabolism 7: 11.

Cheng, H. H. and Lai, M. H. (2000). Fermentation of resistant rice starch produces propionate reducing serum and hepatic cholesterol in rats. Journal of Nutrition 130: 1991-1995.

Chezem, J., Colon, I. and Matheson, H. (1996). Dietary fiber source alters liver cholesterol, bile acid synthesis and excretion in rats. FASEB Journal 10: 2940-2940.

Chiba, H., Uehara, M., Wu, J., Wang, X. X., Masuyama, R., Suzuki, K., Kanazawa, K. and Ishimi, Y. (2003). Hesperidin, a citrus flavonoid, inhibits bone loss and decreases serum and hepatic lipids in ovariectomized mice. Journal of Nutrition 133: 1892-1897. 
Cho, K., Kim, S. J., Park, S. H., Kim, S. and Park, T. (2009a). Protective effect of Codonopsis lanceolata root extract against alcoholic fatty liver in the rat. Journal of Medicinal Food 12 1293-1301.

Cho, S. H., Lee, H. R., Kim, T. H., Choi, S. W., Lee, W. J. and Choi, Y. (2004). Effects of defatted safflower seed extract and phenolic compounds in diet on plasma and liver lipid in ovariectomized rats fed high-cholesterol diets. Journal of Nutritional Science and Vitaminology 50: $32-37$.

Cho, Y. Y., Kwon, E. Y., Kim, H. J., Park, Y. B., Lee, K. T., Park, T. and Choi, M. S. (2009b). Low trans structured fat from flaxseed oil improves plasma and hepatic lipid metabolism in apo E-/mice. Food and Chemical Toxicology 47: 1550-1555.

Chopra, R. and Sambaiah, K. (2009). Effects of rice bran oil enriched with n-3 PUFA on liver and serum lipids in rats. Lipids 44 : 37-46.

Clark, R. M., Balakrishnan, A., Waters, D., Aggarwal, D., Owen, K. Q. and Koo, S. I. (2007). Lcarnitine increases liver alpha-tocopherol and lowers liver and plasma triglycerides in aging ovariectomized rats. Journal of Nutritional Biochemistry 18: 623-628.

Clarke, S. D., Armstrong, M. K. and Jump, D. B. (1990). Dietary polyunsaturated fats uniquely suppress rat liver fatty acid synthase and S14 mRNA content. Journal of Nutrition 120: 225-231.

Clarke, S. D., Romsos, D. R. and Leveille, G. A. (1977). Differential effects of dietary methyl esters of long-chain saturated and polyunsaturated fatty acids on rat liver and adipose tissue lipogenesis. Journal of Nutrition 107: 1170-1181.

Coudé, F. X., Grimber, G., Pelet, A. and Benoit, Y. (1983). Action of the antiepileptic drug, valproic acid, on fatty acid oxidation in isolated rat hepatocytes. Biochemical and Biophysical Research Communications 115: 730-736.

da Costa, K.-A., Garner, S. C., Chang, J. and Zeisel, S. H. (1995). Effects of prolonged (1 year) choline deficiency and subsequent re-feeding of choline on 1,2-sn-diradylglycerol, fatty acids and protein kinase $\mathrm{C}$ in rat liver. Carcinogenesis 16: 327-334.

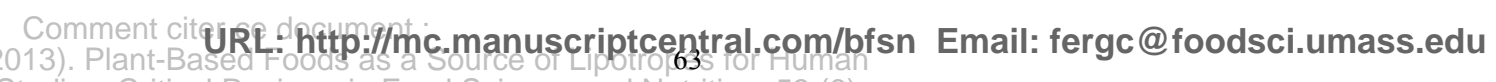
Fardet, A., Chardigny, J.-M. (2013. Putrition. A Survey of In Vivo Studies. Critical Reviews in Food Science and Nutrition, 53 (6)

Nutrition: A Survey of In Vivo Studies. Critical Reviews in Food Science
535-590. DOI: 10.1080/10408398.2010.549596 
Dahiru, D. and Obidoa, O. (2009). Effect of aqueous extract of Ziziphus mauritiana leaf on cholesterol and triglyceride levels in serum and liver of rats administered alcohol. Pakistan Journal of Nutrition 8: 1884-1888.

Daubioul, C., Rousseau, N., Demeure, R., Gallez, B., Taper, H., Declerck, B. and Delzenne, N. (2002). Dietary fructans, but not cellulose, decrease triglyceride accumulation in the liver of obese Zucker fa/fa rats. Journal of Nutrition 132: 967-973

Daubioul, C. A., Taper, H. S., De Wispelaere, L. D. and Delzenne, N. M. (2000). Dietary oligofructose lessens hepatic steatosis, but does not prevent hypertriglyceridemia in obese Zucker rats. Journal of Nutrition 130: 1314-1319.

Décordé, K., Agne, A., Lacan, D., Ramos, J., Fouret, G., Ventura, E., Feillet-Coudray, C., Cristol, J.-P. and Rouanet, J.-M. (2009). Preventive effect of a melon extract rich in superoxide scavenging activity on abdominal and liver fat and adipokine imbalance in high-fat-fed hamsters. Journal of Agricultural and Food Chemistry 57: 6461-6467.

Degrace, P., Demizieux, L., Du, Z. Y., Gresti, J., Caverot, L., Djaouti, L., Jourdan, T., Moindrot, B., Guilland, J. C., Hocquette, J. F. and Clouet, P. (2007). Regulation of lipid flux between liver and adipose tissue during transient hepatic steatosis in carnitine-depleted rats. Journal of Biological Chemistry 282: 20816-20826.

Delzenne, N. M. and Kok, N. N. (1999). Biochemical basis of oligofructose-induced hypolipidemia in animal models. Journal of Nutrition 129: 1467S-1470S.

Demigné, C., Morand, C., Levrat, M.-A., Besson, C., Moundras, C. and Rémésy, C. (1995). Effect of propionate on fatty acid and cholesterol synthesis and on acetate metabolism in isolated rat hepatocytes. British Journal of Nutrition 74: 209-219.

Di Nunzio, M., van Deursen, D., Verhoeven, A. J. M. and Bordoni, A. (2010). n-3 and n-6 Polyunsaturated fatty acids suppress sterol regulatory element binding protein activity and increase flow of non-esterified cholesterol in HepG2 cells. British Journal of Nutrition 103: 161167 
Doan Thi Thanh, H., Takahashi, Y. and Ide, T. (2006). Activity and mRNA levels of enzymes involved in hepatic fatty acid oxidation in mice fed citrus flavonoids. Nutrition 22: 546-552.

Drill, V. A. (1954). Lipotropic effects of vitamin-B12 and other factors. Annals of the New York Academy of Sciences 57: 654-663.

Dumas, M. E., Barton, R. H., Toye, A., Cloarec, O., Blancher, C., Rothwell, A., Fearnside, J., Tatoud, R., Blanc, V., Lindon, J. C., Mitchell, S. C., Holmes, E., McCarthy, M. I., Scott, J., Gauguier, D. and Nicholson, J. K. (2006). Metabolic profiling reveals a contribution of gut microbiota to fatty liver phenotype in insulin-resistant mice. Proceedings of the Nationa Academy of Sciences of the United States of America 103: 12511-12516.

Eckstein, H. C. (1952). Dietary essential amino acids and the liver lipide content of young white rats. Journal of Biological Chemistry 195: 167-174.

Engel, R. W. (1942). The relation of B-vitamins and dietary fat to the lipotropic action of choline. Journal of Nutrition 24: 175-185.

Esfandiari, F., Villanueva, J. A., Wong, D. H., French, S. W. and Halsted, C. H. (2005). Chronic ethanol feeding and folate deficiency activate hepatic endoplasmic reticulum stress pathway in micropigs. American Journal of Physiology-Gastrointestinal and Liver Physiology 289: G54-63.

Failey, R. B. and Childress, R. H. (1962). The effect of para-aminobenzoic acid on the serum cholesterol level in man. American Journal of Clinical Nutrition 10: 158-162.

Feillet-Coudray, C., Sutra, T., Fouret, G., Ramos, J., Wrutnialc-Cabello, C., Cabello, G., Cristol, J.

P. and Coudray, C. (2009). Oxidative stress in rats fed a high-fat high-sucrose diet and preventive effect of polyphenols: Involvement of mitochondrial and $\mathrm{NAD}(\mathrm{P}) \mathrm{H}$ oxidase systems. Free Radical Biology and Medicine 46: 624-632.

Felmlee, M. A., Woo, G., Simko, E., Krol, E. S., Muir, A. D. and Alcorn, J. (2009). Effects of the flaxseed lignans secoisolariciresinol diglucoside and its aglycone on serum and hepatic lipids in hyperlipidaemic rats. British Journal of Nutrition 102: 361-369. 
Fomenko, A. I., Shushevich, S. I. and Khalmuradov, A. G. (1979). Inhibition of activity of acetyl coenzyme A carboxylase from chicken liver by nicotinic-acid and its derivatives. Biokhimiya 44: 1005-1009.

Frantz, I. D. and Carey, J. B. (1961). Cholesterol content of human liver after feeding of corn oil and hydrogenated coconut oil. Proceedings of the Society for Experimental Biology and Medicine 106: $800-801$.

Fritz, I. B. (1959). Action of carnitine on long chain fatty acid oxidation by liver. American Journal of Physiology 197: 297-304.

Fritz, I. B. (1964). Carnitine effects on palmitate-1 $1-^{14} \mathrm{C}$ conversion to $\mathrm{CO}_{2}$ and glycerides by various tissues. American Journal of Physiology 206: 1217-1222.

Fritz, I. B. and Dupont, P. (1957). Ineffectiveness of carnitine as a choline substitute in the prevention of fatty livers of rats maintained on a choline-deficient diet. American Journal of Physiology 190: 453-454.

Fukuda, N., Miyagi, C., Zhang, L., Jayasooriya, A. P., Sakono, M., Yamamoto, K., Ide, T. and Sugano, M. (1998). Reciprocal effects of dietary sesamin on ketogenesis and triacylglycerol secretion by the rat liver. Journal of Nutritional Science and Vitaminology 44: 715-722.

Fukuda, N., Zhang, L., Kodama, M., Sakono, M., Ide, T., Yamamoto, K. and Sugano, M. (1999). Effect of dietary sesamin on metabolic fate of an exogenous linolelaidic acid in perfused rat liver. Journal of Nutritional Science and Vitaminology 45: 437-448.

Fukushima, Y., Kasuga, M., Nakao, K., Shimomura, I. and Matsuzawa, Y. (2009). Effects of coffee on inflammatory cytokine gene expression in mice fed high-fat diets. Journal of Agricultural and Food Chemistry 57: 11100-11105.

Gallaher, D. D. and Plate, A. Y. A. (2005). Reduction in liver cholesterol by a beta-glucan concentrate in rats: correlation with intestinal contents viscosity. FASEB Journal 19: A90-A90. 
Ganji, S. H., Tavintharan, S., Zhu, D., Xing, Y., Kamanna, V. S. and Kashyap, M. L. (2004). Niacin noncompetitively inhibits DGAT2 but not DGAT1 activity in HepG2 cells. Journal of Lipid Research 45: 1835-1845.

Gargini, G. (1951). Comparative experimental research on the lipotropic and hepatoprotective action of inositol, amino acids and lipocaic. Rivista Critica di Clinica Medica 51: 195-214.

Gavin, G. and McHenry, E. W. (1941). The effect of biotin upon fat synthesis and metabolism. Journal of Biological Chemistry 141: 619-625.

Gebhardt, R. and Beck, H. (1996). Differential inhibitory effects of garlic-derived organosulfur compounds on cholesterol biosynthesis in primary rat hepatocyte cultures. Lipids 31: 1269-1276. Gershoff, S. N. and Gottlieb, L. S. (1964). Pantothenic acid deficiency in cats. Journal of Nutrition 82: $135-138$.

Goheen, S., Larkin, E. and Rao, G. (1983). Severe fatty liver in rats fed a fat-free ethanol diet, and its prevention by small amounts of dietary arachidonate. Lipids 18: 285-290.

Gotoh, N., Nagao, K., Onoda, S., Shirouchi, B., Furuya, K., Nagai, T., Mizobe, H., Ichioka, K., Watanabe, H., Yanagita, T. and Wada, S. (2009). Effects of three different highly purified n-3 series highly unsaturated fatty acids on lipid metabolism in C57BL/KsJ-db/db mice. Journal of Agricultural and Food Chemistry 57: 11047-11054.

Griffith, W. H. and Mulford, D. J. (1941). Choline metabolism. VI. Hemorrhagic degeneration and the labile methyl supply. Journal of the American Chemical Society 63: 929-932.

Grundy, S., Mok, H., Zech, L. and Berman, M. (1981). Influence of nicotinic acid on metabolism of cholesterol and triglycerides in man. Journal of Lipid Research 22: 24-36.

Gu, X., Xie, Z., Wang, Q., Liu, G., Qu, Y., Zhang, L., Pan, J., Zhao, G. and Zhang, Q. (2009). Transcriptome profiling analysis reveals multiple modulatory effects of Ginkgo biloba extract in the liver of rats on a high-fat diet. FEBS Journal 276: 1450-1458.

Guo, Y., Wu, G., Su, X., Yang, H. and Zhang, J. (2009). Antiobesity action of a daidzein derivative on male obese mice induced by a high-fat diet. Nutrition Research 29: 656-663.

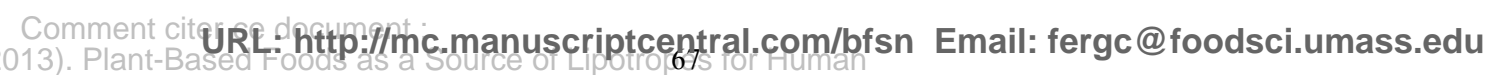
Fardet, A., Chardigny, J.-M. (2013)

Nutrition: A Survey of In Vivo Studies. Critical Reviews in Food Science
535-590. DOI: 10.1080/10408398.2010.549596 
Gustavsson, C., Parini, P., Ostojic, J., Cheung, L., Hu, J., Zadjali, F., Tahir, F., Brismar, K., Norstedt, G. and Tollet-Egnell, P. (2009). Cocoa Butter and Safflower Oil Elicit Different Effects on Hepatic Gene Expression and Lipid Metabolism in Rats. Lipids 44: 1011-1027.

Haines, D. S. M. and Mookerjea, S. (1965). Impairment of triglyceride transport from liver in choline deficiency. Canadian Journal of Biochemistry 43: 507-520.

Haines, D. S. M. and Rose, C. I. (1970). Impaired labelling of liver phosphatidylethanolamine from ethanolamine- $\mathrm{C}^{14}$ in choline deficiency. Canadian Journal of Biochemistry 48: 885-892.

Halsted, C. H., Villanueva, J. A., Devlin, A. M., Niemelä, O., Parkkila, S., Garrow, T. A., Wallock, L. M., Shigenaga, M. K., Melnyk, S. and James, S. J. (2002). Folate deficiency disturbs hepatic methionine metabolism and promotes liver injury in the ethanol-fed micropig. Proceedings of the National Academy of Sciences of the United States of America 99: 10072-10077.

Han, L. K., Sumiyoshi, M., Zhang, J., Liu, M. X., Zhang, X. F., Zheng, Y. N., Okuda, H. and Kimura, Y. (2003). Anti-obesity action of Salix matsudana leaves (Part 1). Anti-obesity action by polyphenols of Salix matsudana in high fat-diet treated rodent animals. Phytotherapy Research 17: $1188-1194$.

Han, L.-K., Xu, B.-J., Kimura, Y., Zheng, Y.-n. and Okuda, H. (2000). Platycodi radix affects Lipid metabolism in mice with high fat diet-induced obesity. Journal of Nutrition 130: 2760-2764.

Hara, H., Haga, S., Aoyama, Y. and Kiriyama, S. (1999). Short-chain fatty acids suppress cholesterol synthesis in rat liver and intestine. Journal of Nutrition 129: 942-948.

Harper, A. E., Benton, D. A., Winje, M. E. and Elvehjem, C. A. (1954). On the lipotropic action of protein. Journal of Biological Chemistry 209: 171-177.

Hartfiel, W. and Kirchner, I. (1973). The importance of nicotinic acid and its effect on the liver fat content of laying hens. Archiv fur Geflugelkunde 37: 114-117.

Hayashi, E., Maeda, T. and Tomita, T. (1974a). Effect of myoinositol deficiency on lipidmetabolism in rats. 1. Alteration of lipid-metabolism in myoinositol deficient rats. Biochimica et Biophysica Acta 360: 134-145. 
Hayashi, E., Maeda, T. and Tomita, T. (1974b). Effect of myoinositol deficiency on lipidmetabolism in rats. 2. Mechanism of triacylglycerol accumulation in liver of myoinositol-deficient rats. Biochimica et Biophysica Acta 360: 146-155.

Hayes, K. C., Pronczuk, A., Cook, M. W. and Robbins, M. C. (2003). Betaine in sub-acute and subchronic rat studies. Food and Chemical Toxicology 41: 1685-1700.

Hayes, K. C., Pronczuk, A., Wijendran, V. and Beer, M. (2002). Free phytosterols effectively reduce plasma and liver cholesterol in gerbils fed cholesterol. Journal of Nutrition 132: 1983 1988.

Hernandez, R., Martinez-Lara, E., Canuelo, A., del Moral, M. L., Blanco, S., Siles, E., Jimenez, A., Pedrosa, J. A. and Peinado, M. A. (2005). Steatosis recovery after treatment with a balanced sunflower or olive oil-based diet: involvement of perisinusoidal stellate cells. World Journal of Gastroenterology 11: 7480-7485.

Hong, S. Y., Kim, Y. J. and Kim, M. K. (2007). Effect of hydroxycitric acid feeding on body weight and lipid profile in rats fed a high-carbohydrate or high-fat diet. Faseb Journal 21: A1055A1055.

Hood, R. L. and Sidhu, G. S. (1992). Effect of guar gum and tocotrienols on cholesterol metabolism on the Japanese quail. Nutrition Research 12: S117-S127.

Hosein, E. A. and Bexton, B. (1975). Protective action of carnitine on liver lipid-metabolism after ethanol administration to rats. Biochemical Pharmacology 24: 1859-1863.

Hotta, K., Kuwajima, M., Ono, A., Uenaka, R., Nakajima, H., Miyagawa, J., Namba, M., Hanafusa, T., Horiuchi, M., Nikaido, H., Hayakawa, J., Kono, N., Saheki, T. and Matsuzawa, Y. (1996). Altered expression of carnitine palmitoyltransferase II in liver, muscle, and heart of mouse strain with juvenile visceral steatosis. Biochimica et Biophysica Acta-General Subjects 1289: 131-135.

Hou, Z., Qin, P. and Ren, G. (2010). Effect of anthocyanin-rich extract from black rice (Oryza sativa L. Japonica) on chronically alcohol-induced liver damage in rats. Journal of Agricultural and Food Chemistry. 
Hsu, C.-c., Yen, H.-f., Yin, M.-c., Tsai, C.-m. and Hsieh, C.-h. (2004). Five cysteine-containing compounds delay diabetic deterioration in Balb/cA mice. Journal of Nutrition 134: 3245-3249.

Hu, M. C. C. (1975). Lipotropic action of carnitine in a low protein diet from plant sources. Dissertation Abstracts International, В 36: 655.

Hurley, L. S., Volkert, N. E. and Eichner, J. T. (1965). Pantothenic acid deficiency in pregnant and non-pregnant guinea pigs, with special reference to effects on the fetus. Journal of Nutrition 86: 201-208.

Hussein, O., Grosovski, M., Lasri, E., Svalb, S., Ravid, U. and Assy, N. (2007). Monounsaturated fat decreases hepatic lipid content in non-alcoholic fatty liver disease in rats. World Journal of Gastroenterology 13: 361-368.

Ide, T., Ashakumary, L., Takahashi, Y., Kushiro, M., Fukuda, N. and Sugano, M. (2001). Sesamin, a sesame lignan, decreases fatty acid synthesis in rat liver accompanying the down-regulation of sterol regulatory element binding protein-1. Biochimica et Biophysica Acta-Molecular and Cell Biology of Lipids 1534: 1-13.

Ide, T., Hong, D. D., Ranasinghe, P., Takahashi, Y., Kushiro, M. and Sugano, M. (2004). Interaction of dietary fat types and sesamin on hepatic fatty acid oxidation in rats. Biochimica et Biophysica Acta-Molecular and Cell Biology of Lipids 1682: 80-91.

Ide, T. and Horii, M. (1989). Hepatic carnitine and triglyceride lowering effect of dietary pectin in the rat. Nutrition Reports International 39: 861-865.

Ikeda, M., Uno, Y., Iwai, M., Sato, H., Kawabe, H. and Sakakibara, B. (1992). Effects of the inositol-deficient diet on the development of fatty liver lipogenic enzyme activities and plasma lipid levels in germ-free and conventional mice. Vitamins (Kyoto) 66: 43-49.

Itokawa, Y., Inoue, K., Sasagawa, S. and Fujiwara, M. (1973). Effect of s-methylcysteine sulfoxide, s-allylcysteine sulfoxide and related sulfur-containing amino acids on lipid metabolism of experimental hypercholesterolemic rats. Journal of Nutrition 103: 88-92. 
Jackson, K. A., Suter, D. A. I. and Topping, D. L. (1994). Oat bran, barley and malted barley lower plasma-cholesterol relative to wheat bran but differ in their effects on liver cholesterol in rats fed diets with and without cholesterol. Journal of Nutrition 124: 1678-1684.

Ji, C. and Kaplowitz, N. (2003). Betaine decreases hyperhomocysteinemia, endoplasmic reticulum stress, and liver injury in alcohol-fed mice. Gastroenterology 124: 1488-1499.

Jin, F. Y., Kamanna, V. S. and Kashyap, M. L. (1999). Niacin accelerates intracellular apoB degradation by inhibiting triacylglycerol synthesis in human hepatoblastoma (HepG2) cells. Arteriosclerosis Thrombosis and Vascular Biology 19: 1051-1059.

Jin, F.-Y., Kamanna, V. S. and Kashyap, M. L. (1997). Niacin decreases removal of high-density lipoprotein apolipoprotein A-I but not cholesterol ester by Hep G2 cells : Implication for Reverse Cholesterol Transport. Arteriosclerosis, Thrombosis and Vascular Biology 17: 2020-2028. Jonnalagadda, S. S., Thye, F. W. and Robertson, J. L. (1993). Plasma total and lipoprotein cholesterol, liver cholesterol and fecal cholesterol excretion in hamsters fed fiber diets. Journal of Nutrition 123: 1377-1382.

Kahlon, T. S., Chow, F. I., Sayre, R. N. and Betschart, A. A. (1992a). Cholesterol-lowering in hamsters fed rice bran at various levels, defatted rice bran and rice bran oil. Journal of Nutrition 122: $513-519$.

Kahlon, T. S., Saunders, R. M., Sayre, R. N., Chow, F. I., Chiu, M. M. and Betschart, A. A. (1992b). Cholesterol-lowering effects of rice bran and rice bran oil fractions in hypercholesterolemic hamsters. Cereal Chemistry 69: 485-489.

Kamal-Eldin, A., Frank, J., Razdan, A., Tengblad, S., Basu, S. and Vessby, B. (2000). Effects of dietary phenolic compounds on tocopherol, cholesterol, and fatty acids in rats. Lipids 35: 427435

Kanuri, G., Weber, S., Volynets, V., Spruss, A., Bischoff, S. C. and Bergheim, I. (2009). Cinnamon extract protects against acute alcohol-induced liver steatosis in mice. Journal of Nutrition 139: $482-487$ 
Karanth, J. and Jeevaratnam, K. (2009). Effect of dietary lipid, carnitine and exercise on lipid profile in rat blood, liver and muscle. Indian Journal of Experimental Biology 47: 748-753.

Katagiri, M. and Shimizu, S. (1992). Effects of dietary phytosterols on cholesterol levels in liver, serum and faeces of rats (a preliminary report). Bulletin of IIDA Women's Junior College: 60-67.

Katayama, T. (1993). Effect of dietary addition of myo-inositol on the metabolic changes in rats exposed to 1,1,1-trichloro-2, 2-bis (P-chlorophenyl) ethane. Nutrition Research 13: 445-454.

Katayama, T. (1994). Effect of dietary addition of myo-inositol on lipid metabolism in rats fed sucrose or corn starch. Nutrition Research 14: 699-706.

Katayama, T. (1995). Effect of dietary-sodium phytate on the hepatic and serum levels of lipids and on the hepatic activities of NADPH-generating enzymes in rats fed on sucrose. Bioscience, Biotechnology and Biochemistry 59: 1159-1160.

Katayama, T. (1997a). Dietary phytic acid acts on hepatic lipid metabolism in a similar manner as dietary myo-inositol: is phytic acid a vitamin-like substance? Recent Research Developments in Agricultural and Biological Chemistry 1: 321-330.

Katayama, T. (1997b). Effects of dietary myo-inositol or phytic acid on hepatic concentrations of lipids and hepatic activities of lipogenic enzymes in rats fed on corn starch or sucrose. Nutrition Research 17: 721-728.

Katz, M., Budowski, P. and Bondi, A. (1970). Inhibition of cholesterol deposition in livers of mice fed phytosterols in short-term experiments. Journal of Nutrition 100: 1141-1147.

Kawakami, Y., Tsurugasaki, W., Nakamura, S. and Osada, K. (2005). Comparison of regulative functions between dietary soy isoflavones aglycone and glucoside on lipid metabolism in rats fed cholesterol. Journal of Nutritional Biochemistry 16: 205-212.

Kelley, B., Totter, J. R. and Day, P. L. (1950). The lipotropic effect of folic acid on rats receiving various purified diets. Journal of Biological Chemistry 187: 529-535.

Kelley, J. J. and Tsai, A. C. (1978). Effect of pectin, gum arabic and agar on cholesterol absorption, synthesis, and turnover in rats. Journal of Nutrition 108: 630-639.

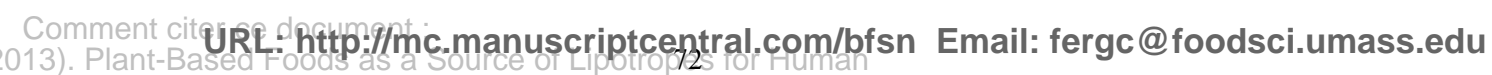
Fardet, A., Chardigny, J.-M. (2013). Plant-Based Fiews in Food Science and Nutrition, 53 (6),

Nutrition: A Survey of In Vivo Studies. Critical Reviews in Food Science
535-590. DOI : 10.1080/10408398.2010.549596 
Khairallah, E. A. and Wolf, G. (1965). Growth-promoting and lipotropic effect of carnitine in rats fed diets limited in protein and methionine. Journal of Nutrition 87: 469-476.

Khanal, T., Choi, J. H., Hwang, Y. P., Chung, Y. C. and Jeong, H. G. (2009a). Protective effects of saponins from the root of Platycodon grandiflorum against fatty liver in chronic ethanol feeding via the activation of AMP-dependent protein kinase. Food and Chemical Toxicology 47: 27492754.

Khanal, T., Choi, J. H., Hwang, Y. P., Chung, Y. C. and Jeong, H. G. (2009b). Saponins isolated from the root of Platycodon grandiflorum protect against acute ethanol-induced hepatotoxicity in mice. Food and Chemical Toxicology 47: 530-535.

Kharbanda, K. K., Rogers, D. D., Mailliard, M. E., Siford, G. L., Barak, A. J., Beckenhauer, H. C., Sorrell, M. F. and Tuma, D. J. (2005). A comparison of the effects of betaine and Sadenosylmethionine on ethanol-induced changes in methionine metabolism and steatosis in rat hepatocytes. Journal of Nutrition 135: 519-524.

Khor, H. T., Chieng, D. Y. and Ong, K. K. (1995). Tocotrienols inhibit liver HMG CoA reductase activity in the guinea pig. Nutrition Research 15: 537-544.

Khor, H. T. and Ng, T. T. (2000). Effects of administration of alpha-tocopherol and tocotrienols on serum lipids and liver HMG CoA reductase activity. International Journal of Food Sciences and Nutrition 51: S3-S11.

Kim, D. Y., Park, J. S., Yuan, H. D. and Chung, S. H. (2009). Fermented ginseng attenuates hepatic lipid accumulation and hyperglycemia through AMPK activation. Food Science and Biotechnology 18: 172-178.

Kim, H., Bartley, G. E., Rimando, A. M. and Yokoyama, W. (2010). HHepatic gene expression related to lower plasma cholesterol in hamsters fed high-fat diets supplemented with blueberry peels and peel extract. Journal of Agricultural and Food Chemistry.

Kim, H. K., Jeong, T. S., Lee, M. K., Park, Y. B. and Choi, M. S. (2003). Lipid-lowering efficacy of hesperetin metabolites in high-cholesterol fed rats. Clinica Chimica Acta 327: 129-137.

Comment cit巳RE :http:f/mc.manuscriptcentral.com/bfsn Email: fergc@foodsci.umass.edu Fardet, A., Chardigny, J.-M. (2013). Plantt-Basd

Nutrition: A Survey of In Vivo Studies. Critical Reviews in Food Science
535-590. DOI: 10.1080/10408398 2010.549596 
Kim, S., Shin, H. J., Kim, S. Y., Kim, J. H., Lee, Y. S., Kim, D. H. and Lee, M. O. (2004). Genistein enhances expression of genes involved in fatty acid catabolism through activation of PPARalpha. Molecular and Cellular Endocrinology 220: 51-58.

Kim, S., Sohn, I. and Lee, Y. S. (2005). Hepatic gene expression profiles are altered by genistein supplementation in mice with diet-induced obesity. Journal of Nutrition 135: 33-41.

Klopfenstein, C. F. (1990). Nutritional properties of coarse and fine sugar-beet fiber and hard red wheat bran. 1. Effects on rat serum and liver cholesterol and triglycerides and on fecal characteristics. Cereal Chemistry 67: 538-541.

Kok, N., Roberfroid, M. and Delzenne, N. (1996a). Dietary oligofructose modifies the impact of fructose on hepatic triacylglycerol metabolism. Metabolism 45: 1547-1550.

Kok, N., Roberfroid, M., Robert, A. and Delzenne, N. (1996b). Involvement of lipogenesis in the lower VLDL secretion induced by oligofructose in rats. British Journal of Nutrition 76: 881-890. Kok, N. N., Taper, H. S. and Delzenne, N. M. (1998). Oligofructose modulates lipid metabolism alterations induced by a fat-rich diet in rats. Journal of Applied Toxicology 18: 47-53.

Kondo, T., Kishi, M., Fushimi, T. and Kaga, T. (2009). Acetic acid upregulates the expression of genes for fatty acid oxidation enzymes in liver to suppress body fat accumulation. Journal of Agricultural and Food Chemistry 57: 5982-5986.

Kotaki, A., Sakurai, T., Kobayashi, M. and Yagi, K. (1968). Studies on myoinositol. IV. Effect of myoinositol on the cholesterol metabolism of rats suffering from experimental fatty liver. Journal of Vitaminology (Kyoto) 14.

Kritchevsky, D. and Tepper, S. A. (2005). Influence of a fiber mixture on serum and liver lipids and on fecal fat excretion in rats. Nutrition Research 25: 485-489.

Kritchevsky, D., Tepper, S. A., Satchithanandam, S., Cassidy, M. M. and Vahouny, G. V. (1988) Influence of a fiber mixture on serum and liver lipids and on fecal fat excretion in rats. Lipids 23: 318-321. 
Krogh, B., Funch, J. P. and Dam, H. (1961). Cholesterol and polyenoic fatty acids in liver, serum and aorta of rabbits given purified diets with butter, some margarines and arachis oil. British Journal of Nutrition 15: 481-488.

Kumari, K. and Augusti, K. T. (2007). Lipid lowering effect of S-methyl cysteine sulfoxide from Allium cepa Linn in high cholesterol diet fed rats. Journal of Ethnopharmacology 109: 367-371.

Kumari, K., Mathew, B. C. and Augusti, K. T. (1995). Antidiabetic and hypolipidemic effects of Smethyl cysteine sulfoxide isolated from Allium cepa linn. Indian Journal of Biochemistry and Biophysics 32: 49-54.

Kuroki, S., Muramoto, S., Kuramoto, T. and Hoshita, T. (1983). Effect of feeding cholesterol and sitosterol on hepatic-steroid 12-alpha-hydroxylase activity in female hamsters. Journal of Pharmacobio-Dynamics 6: 551-557.

Kushiro, M., Masaoka, T., Hageshita, S., Takahashi, Y., Ide, T. and Sugano, M. (2002) Comparative effect of sesamin and episesamin on the activity and gene expression of enzymes in fatty acid oxidation and synthesis in rat liver. Journal of Nutritional Biochemistry 13: 289-295. Kushiro, M., Takahashi, Y. and Ide, T. (2004). Species differences in the physiological activity of dietary lignan (sesamin and episesamin) in affecting hepatic fatty acid metabolism. British Journal of Nutrition 91: 377-386.

Kuzu, N., Bahcecioglu, I. H., Metin, K., Ozercan, I. H., Tuzcu, M., Yalniz, M., Ustundag, B. and Sahin, K. (2007). Role of melatonin in treatment of nonalcoholic steatohepatitis in rats induced bu high fat diet. Journal of Hepatology 46: S263.

Kwon, D. Y., Kim, Y. S., Hong, S. M. and Park, S. (2009). Long-term consumption of saponins derived from Platycodi radix (22 years old) enhances hepatic insulin sensitivity and glucosestimulated insulin secretion in $90 \%$ pancreatectomized diabetic rats fed a high-fat diet. British Journal of Nutrition 101: 358-366.

Laird, R. D. and Drill, V. A. (1971). Lipotropic activity of inositol and chlortetracycline alone and in various combinations of choline, vitamin $\mathrm{B}_{12}$ and folic acid - activity of 3 liver extracts with 
assays for these substances. Archives Internationales de Pharmacodynamie et de Thérapie 194:

103-116.

Laird, R. D., McCormick, H. M. and Drill, V. A. (1965). Lipotropic activity of combinations of choline, vitamins $\mathrm{B}_{12}$ and $\mathrm{B}_{12 \mathrm{~b}}$, folic acid, and citrovorum factor. Toxicology and Applied Pharmacology 7: 247-256.

Lamon-Fava, S., Diffenderfer, M. R., Barrett, P. H. R., Buchsbaum, A., Nyaku, M., Horvath, K. V., Asztalos, B. F., Otokozawa, S., Ai, M., Matthan, N. R., Lichtenstein, A. H., Dolnikowski, G. G. and Schaefer, E. J. (2008). Extended-release niacin alters the metabolism of plasma apolipoprotein (Apo) A-I and apoB-containing lipoproteins. Arteriosclerosis Thrombosis and Vascular Biology 28: 1672-1678.

Laraki, L., Pelletier, X., Mourot, J. and Debry, G. (1993). Effects of dietary phytosterols on liver lipids and lipid metabolism enzymes. Annals of Nutrition and Metabolism 37: 129-133.

Leclerc, J. and Miller, M. L. (1989). Inositol and choline levels in the diet and neutral lipid hepatic content of lactating rat. International Journal for Vitamin and Nutrition Research 59: 180-183.

Lee, S. H., Park, H. J., Cho, S. Y., Jung, H. J., Cho, S. M., Cho, Y. S. and Lillehoj, H. S. (2005). Effects of dietary phytic acid on serum and hepatic lipid levels in diabetic KK mice. Nutrition Research 25: 869-876.

Lee, S.-H., Park, H.-J., Chun, H.-K., Cho, S.-Y., Jung, H.-J., Cho, S.-M., Kim, D.-Y., Kang, M.-S. and Lillehoj, H. S. (2007). Dietary phytic acid improves serum and hepatic lipid levels in aged ICR mice fed a high-cholesterol diet. Nutrition Research 27: 505-510.

Lee, Y. H. and Yeh, Y. Y. (2003). Inhibitory effects of garlic extract and water-soluble organosulfur compounds of garlic on cholestergenesis in HepG-2 cells. FASEB Journal 17: A752-A752.

Li, B. H. and Tian, W. X. (2004). Inhibitory effects of flavonoids on animal fatty acid synthase. Journal of Biochemistry 135: 85-91. 
Li, R., Jia, C.-S., Yue, L., Zhang, X.-M., Xia, Q.-Y., Zhao, S.-L., Feng, B., Zhong, F. and Chen,

W.-J. (2010). Lipase-catalyzed synthesis of conjugated linoleyl $\beta$-Sitosterol and its cholesterollowering properties in mice. Journal of Agricultural and Food Chemistry 58: 1898-1902.

Liang, L. J., Yin, X. Y., Luo, S. M., Zheng, J. F., Lu, M. D. and Huang, J. F. (1999). A study of the ameliorating effects of carnitine on hepatic steatosis induced by total parenteral nutrition in rats. World Journal of Gastroenterology 5: 312-315.

Lim, J. S., Adachi, Y., Takahashi, Y. and Ide, T. (2007). Comparative analysis of sesame lignans (sesamin and sesamolin) in affecting hepatic fatty acid metabolism in rats. British Journal of Nutrition 97: 85-95

Lin, C. C. and Yin, M. C. (2008). Effects of cysteine-containing compounds on biosynthesis of triacylglycerol and cholesterol and anti-oxidative protection in liver from mice consuming a highfat diet. British Journal of Nutrition 99: 37-43.

Lin, C. C., Yin, M. C., Hsu, C. C. and Lin, M. P. (2004). Effect of five cysteine-containing compounds on three lipogenic enzymes in Balb/cA mice consuming a high saturated fat diet. Lipids 39: 843-848.

Lin, C. C., Yin, M. C. and Liu, W. H. (2008). Alleviative effects of s-allyl cysteine and s-ethyl cysteine on MCD diet-induced hepatotoxicity in mice. Food and Chemical Toxicology 46: 34013406.

Lin, M., Kao, S., Chung, P., Chan, K., Yang, M. and Wang, C. (2009). Improvement for high fat diet-induced hepatic injuries and oxidative stress by flavonoid-enriched extract from Nelumbo nucifera leaf. Journal of Agricultural and Food Chemistry 57: 5925-5932.

Ling, W. H. and Jones, P. J. H. (1995). Enhanced efficacy of sitostanol-containing versus sitostanolfree phytosterol mixtures in altering lipoprotein cholesterol levels and synthesis in rats. Atherosclerosis 118: 319-331.

Liu, L. and Yeh, Y.-Y. (2000). Inhibition of cholesterol biosynthesis by organosulfur compounds derived from garlic. Lipids 35: 197-203.

Comment citURL Fardet, A., Chardigny, J.-M. (2013). PlanttBastions

Nutrition: A Survey of In Vivo Studies. Critical Reviews in Food Science
535-590. DOI: 10.1080/10408398.2010.549596 
Lombardi, B., Pani, P. and Schlunk, F. F. (1968). Choline-deficiency fatty liver: impaired release of hepatic triglycerides. Journal of Lipid Research 9: 437-446.

Looney, M. A. and Lei, K. Y. (1978). Dietary fiber, zinc and copper - effects on serum and liver cholesterol levels in rat. Nutrition Reports International 17: 329-337.

Lowenstein, J. M. (1971). Effect of (-)-hydroxycitrate on fatty acid synthesis by rat liver in vivo. Journal of Biological Chemistry 246: 629-632.

Luo, Q.-F., Sun, L., Si, J.-Y. and Chen, D.-H. (2008). Hypocholesterolemic effect of stilbenes containing extract-fraction from Cajanus cajan L. on diet-induced hypercholesterolemia in mice. Phytomedicine 15: 932-939.

Mahboob, S. (1975). Effect of pantothenic-acid deficiency on microsomal lipids of rat-liver. Annals of Nutrition and Metabolism 19: 91-95.

McGarry, J. D. and Foster, D. W. (1979). In support of the roles of malonyl-CoA and carnitine acyltransferase I in the regulation of hepatic fatty acid oxidation and ketogenesis. Journal of Biological Chemistry 254: 8163-8168.

McHenry, E. W. and Patterson, J. M. (1944). Lipotropic factors. Physiological Reviews 24: 128167.

McNeil, C. J., Hay, S. M., Rucklidge, G. J., Reid, M. D., Duncan, G. J. and Rees, W. D. (2009). Maternal diets deficient in folic acid and related methyl donors modify mechanisms associated with lipid metabolism in the fetal liver of the rat. British Journal of Nutrition 102: 1445-1452.

Menendez, R., Amor, A. M., Gonzalez, R. M., Fraga, V. and Mas, R. (1996). Effect of policosanol on the hepatic cholesterol biosynthesis of normocholesterolemic rats. Biological Research 29: 253-257.

Menendez, R., Arruzazabala, L., Mas, R., DelRio, A., Amor, A. M., Gonzalez, R. M., Carbajal, D., Fraga, V., Molina, V. and Illnait, J. (1997). Cholesterol-lowering effect of policosanol on rabbits with hypercholesterolaemia induced by a wheat starch-casein diet. British Journal of Nutrition 77: 923-932. 
Merrill, J. M. and Lemley-Stone, J. (1957). Effects of nicotinic acid on serum and tissue cholesterol in rabbits. Circulation Research 5: 617-619.

Mezei, O., Banz, W. J., Steger, R. W., Peluso, M. R., Winters, T. A. and Shay, N. (2003). Soy isoflavones exert antidiabetic and hypolipidemic effects through the PPAR pathways in obese Zucker rats and murine RAW 264.7 cells. Journal of Nutrition 133: 1238-1243.

Moghadasian, M. H., Nguyen, L. B., Shefer, S., Salen, G., Batta, A. K. and Frohlich, J. J. (2001) Hepatic cholesterol and bile acid synthesis, low-density lipoprotein receptor function, and plasma and fecal sterol levels in mice: Effects of apolipoprotein E deficiency and probucol or phytostero treatment. Metabolism-Clinical and Experimental 50: 708-714.

Moiseenok, A. G., Sheibak, V. M. and Gurinovich, V. A. (1987). Hepatic CoA, s-acyl-CoA, biosynthetic precursors of the coenzyme and pantothenate-protein complexes in dietary pantothenic-acid deficiency. International Journal for Vitamin and Nutrition Research 57: 71-77. Moon, Y. S., Latasa, M. J., Griffin, M. J. and Sul, H. S. (2002). Suppression of fatty acid synthase promoter by polyunsaturated fatty acids. Journal of Lipid Research 43: 691-698.

Morise, A., Mourot, J., Boué, C., Combe, N., Amsler, G., Gripois, D., Quignard-Boulangé, A., Yvan-Charvet, L., Fénart, E., Weill, P. and Hermier, D. (2006). Gender-related response of lipid metabolism to dietary fatty acids in the hamster. British Journal of Nutrition 95: 709-720.

Morise, A., Thomas, C., Landrier, J.-F., Besnard, P. and Hermier, D. (2009). Hepatic lipid metabolism response to dietary fatty acids is differently modulated by PPAR $\alpha$ in male and female mice. European Journal of Nutrition 48: 465-473.

Mullen, E., Brown, R. M., Osborne, T. F. and Shay, N. F. (2004). Soy isoflavones affect sterol regulatory element binding proteins (SREBPs) and SREBP-regulated genes in HepG2 cells. Journal of Nutrition 134: 2942-2947.

Nagiel-Ostaszewski, I. and Lau-Cam, C. A. (1990). Protection by pantethine, pantothenic-acid and cystamine against carbon tetrachloride-induced hepatotoxicity in the rat. Research Communications in Chemical Pathology and Pharmacology 67: 289-292. 
Nakamura, Y., Kaihara, A., Yoshii, K., Tsumura, Y., Ishimitsu, S. and Tonogai, Y. (2001). Effects of the oral administration of green tea polyphenol and tannic acid on serum and hepatic lipid contents and fecal steroid excretion in rats. Journal of Health Science 47: 107-117.

Nakamura, Y., Kanazawa, M., Liyanage, R., Iijima, S., Han, K. H., Shimada, K., Sekikawa, M., Yamauchi, A., Hashimoto, N., Ohba, K. and Fukushima, M. (2009). Effect of white wheat bread containing sugar beet fiber on serum lipids and hepatic mRNA in rats fed on a cholesterol-free diet. Bioscience, Biotechnology and Biochemistry 73: 1280-1285.

Nakamura, Y. and Tonogai, Y. (2002). Effects of grape seed polyphenols on serum and hepatic lipid contents and fecal steroid excretion in normal and hypercholesterolemic rats. Journal of Health Science 48: 570-578.

Naruta, E. and Buko, V. (2001). Hypolipidemic effect of pantothenic acid derivatives in mice with hypothalamic obesity induced by aurothioglucose. Experimental and Toxicologic Pathology 53: 393-398.

Nieminen, P., Kakela, R., Mustonen, A. M., Hyvarinen, H. and Asikainen, J. (2001). Exogenous melatonin affects lipids and enzyme activities in mink (Mustela vison) liver. Comparative Biochemistry and Physiology C-Toxicology and Pharmacology 128: 203-211.

Noda, S., Haratake, J., Sasaki, A., Ishii, N., Umezaki, H. and Horie, A. (1991). Acute encephalopathy with hepatic steatosis induced by pantothenic-acid antagonist, calcium hopantenate, in dogs. Liver 11: 134-142.

Noh, J.-R., Kim, Y.-H., Gang, G.-T., Yang, K.-J., Lee, H.-S., Nguyen, P. H., Oh, W.-K., Song, K.S. and Lee, C.-H. (2010). Chestnut (Castanea crenata) inner shell extract inhibits development of hepatic steatosis in C57BL/6 mice fed a high-fat diet. Food Chemistry 121: 437-442.

Ntanios, F. Y. and Jones, P. J. H. (1998a). Dietary sitostanol reduces cholesterol absorption and increases cholesterol synthesis in male hamsters. FASEB Journal 12: 4739. 
Ntanios, F. Y. and Jones, P. J. H. (1998b). Effects of variable dietary sitostanol concentrations on plasma lipid profile and phytosterol metabolism in hamsters. Biochimica et Biophysica ActaLipids and Lipid Metabolism 1390: 237-244.

Ntanios, F. Y., Jones, P. J. H. and Frohlich, J. J. (1998a). Dietary sitostanol reduces plaque formation but not lecithin cholesterol acyl transferase activity in rabbits. Atherosclerosis $\mathbf{1 3 8}$ $101-110$.

Ntanios, F. Y., MacDougall, D. E. and Jones, P. J. H. (1998b). Gender effects of tall oil versus soybean phytosterols as cholesterol-lowering agents in hamsters. Canadian Journal of Physiology and Pharmacology 76: 780-787.

Nunn, S. L., Tauxe, W. N. and Juergens, J. L. (1961). Effect of nicotinic acid on human cholesterol biosynthesis. Circulation 24: 1099

Oakenfull, D. G., Fenwick, D. E., Hood, R. L., Topping, D. L., Illman, R. L. and Storer, G. B. (1979). Effects of saponins on bile-acids and plasma-lipids in the rat. British Journal of Nutrition 42: 209-216.

Oda, T., Aoe, S., Sanada, H. and Ayano, Y. (1993). Effects of soluble and insoluble fiber preparations isolated from oat, barley, and wheat on liver cholesterol accumulation in cholesterolfed rats. Journal of Nutritional Science and Vitaminology 39: 73-79.

Odbayar, T. O., Badamhand, D., Kimura, T., Takahashi, Y., Tsushida, T. and Ide, T. (2006). Comparative studies of some phenolic compounds (quercetin, rutin, and ferulic acid) affecting hepatic fatty acid synthesis in mice. Journal of Agricultural and Food Chemistry 54: 8261-8265.

Okazaki, Y. and Katayama, T. (2003). Effects of dietary carbohydrate and myo-inositol on metabolic changes in rats fed 1,1,1-trichloro-2,2-bis (p-chlorophenyl) ethane (DDT). The Journal of Nutritional Biochemistry 14: 81-89.

Okazaki, Y. and Katayama, T. (2008). Dietary myositol hexakisphosphate, but not myo-inositol, clearly improves hypercholesterolemia in rats fed casein-type amino acid mixtures and 1,1,1trichloro-2,2-bis (p-chlorophenyl) ethane. Nutrition Research 28: 714-721. 
Okazaki, Y., Kayashima, T. and Katayama, T. (2003). Effect of dietary phytic acid on hepatic activities of lipogenic and drug-metabolizing enzymes in rats fed 1,1,1-trichloro-2,2-bis (Pchlorophenyl) ethane (DDT). Nutrition Research 23: 1089-1096.

Okazaki, Y., Setoguchi, T. and Katayama, T. (2006). Effects of dietary myo-inositol, D-chiroinositol and L-chiro-inositol on hepatic lipids with reference to the hepatic myo-inositol status in rats fed on 1,1,1-trichloro-2,2-bis (p-chlorophenyl) ethane. Bioscience, Biotechnology, and Biochemistry 70: 2766-2770.

Okey, R., Miljanich, P., Shannon, A., Tinoco, J. and Ostwald, R. (1961). Fatty acid components of rat liver lipids: effect of composition of diet and of restricted access to food. Journal of Nutrition 75: $51-60$.

Olson, R. E., Jablonski, J. R. and Taylor, E. (1958). The effect of dietary protein, fat, and choline upon the serum lipids and lipoproteins of the rat. American Journal of Clinical Nutrition 6: 111118.

Onning, G. and Asp, N. G. (1995). The effect of dietary protein, fat, and choline upon the serum lipids and lipoproteins of the rat. British Journal of Nutrition 73: 275-286.

Onomi, S. and Katayama, T. (1997). Effects of dietary myo-inositol and phytic acid on hepatic lipids accumulation in rats fed on sucrose or orotic acid. Nippon Eiyo Shokuryo Gakkaishi (Journal of the Japanese Society of Nutrition and Food Science) 50: 267-272.

Ortega, M. F. (1989). Effect of dietary lysine level and protein restriction on the lipids and carnitine levels in the liver of pregnant rats. Annals of Nutrition and Metabolism 33: 162-169.

Osumi, Y., Nagasaka, Y. and Shimamot.K (1969). Lipid metabolism in rats with fatty liver caused by low protein diet and effects of oral administration of L-methionine L-cysteine pantethine and calcium pantothenate upon it. Japanese Journal of Pharmacology 19: 74-88.

Owens (1942). The comparative effects of inositol and lipocaic in depancreatized dogs. Federation Proceedings 1: 65. 
Ozturk, F., Gul, M., Ates, B., Ozturk, I. C., Cetin, A., Vardi, N., Otlu, A. and Yilmaz, I. (2009). Protective effect of apricot (Prunus armeniaca L.) on hepatic steatosis and damage induced by carbon tetrachloride in Wistar rats. British Journal of Nutrition 102: 1767-1775.

Page, G. I., Russell, P. M. and Davies, S. J. (2005). Dietary carotenoid pigment supplementation influences hepatic lipid and mucopolysaccharide levels in rainbow trout (Oncorhynchus mykiss). Comparative Biochemistry and Physiology B-Biochemistry and Molecular Biology 142: 398-402. Pan, M., Song, Y.-L., Xu, J.-M. and Gan, H.-Z. (2006). Melatonin ameliorates nonalcoholic fatty liver induced by high-fat diet in rats. Journal of Pineal Research 41: 79-84.

Park, J. K., Lee, M. A., Chon, J. W., Wang, S. G. and Park, Y. K. (2009a). Effect of water extract of cauliflower mushroom on serum and hepatic lipids in $\mathrm{C} 57 \mathrm{BL} / 6 \mathrm{~J}(\mathrm{OB} / \mathrm{OB})$ mice. Annals of Nutrition and Metabolism 55: 397-398.

Park, K. W., Lee, J.-E. and Park, K.-m. (2009b). Diets containing Sophora japonica L. prevent weight gain in high-fat diet-induced obese mice. Nutrition Research 29: 819-824.

Parker, R. A., Pearce, B. C., Clark, R. W., Gordon, D. A. and Wright, J. J. (1993). Tocotrienols regulate cholesterol production in mammalian cells by post-transcriptional suppression of 3hydroxy-3-methylglutaryl-coenzyme A reductase. Journal of Biological Chemistry 268: 1123011238.

Parsons, W. B. J. (1961). Reduction in hepatic synthesis of cholesterol from $\mathrm{C}^{14}$-acetate in hypercholesterolemic patients by nicotinic acid. Circulation 24: 1099-1100

Pathirana, C., Gibney, M. J. and Taylor, T. G. (1980). Effects of soy protein and saponins on serum and liver cholesterol in rats. Atherosclerosis 36: 595-596.

Peluso, M. R., Winters, T. A., Shanahan, M. F. and Banz, W. J. (2000). A cooperative interaction between soy protein and its isoflavone-enriched fraction lowers hepatic lipids in male obese Zucker rats and reduces blood platelet sensitivity in male Sprague-Dawley rats. Journal of Nutrition 130: 2333-2342. 
Perrault, M. and Dormard, Y. (1966). Lipotropic effect of betaine aspartate on experimental hepatic steatosis. Study using triolein-C ${ }^{14}$. Thérapie 21: 719-731.

Perry, W. F. (1960). Effect of nicotinic acid and nicotinamide on incorporation of acetate into cholesterol, fatty acids and $\mathrm{CO}_{2}$ by rat liver slices. Metabolism-Clinical and Experimental 9: 686689.

Preuss, H. G., Bagchi, D., Bagchi, M., Rao, C. V. S., Dey, D. K. and Satyanarayana, S. (2004a). Effects of a natural extract of (-)-hydroxycitric acid (HCA-SX) and a combination of HCA-SX plus niacin-bound chromium and Gymnema sylvestre extract on weight loss. Diabetes, Obesity and Metabolism 6: 171-180.

Preuss, H. G., Bagchi, D., Bagchi, M., Rao, C. V. S., Satyanarayana, S. and Dey, D. K. (2004b). Efficacy of a novel, natural extract of (-)-hydroxycitric acid (HCA-SX) and a combination of HCA-SX, niacin-bound chromium and Gymnema sylvestre extract in weight management in human volunteers: A pilot study. Nutrition Research 24: 45-58.

Pyo, Y.-H. and Seong, K.-S. (2009). Hypolipidemic effects of monascus-fermented soybean extracts in rats fed a high-fat and -cholesterol diet. Journal of Agricultural and Food Chemistry 57: $8617-8622$.

Qureshi, A. A., Burger, W. C., Peterson, D. M. and Elson, C. E. (1986). The structure of an inhibitor of cholesterol biosynthesis isolated from barley. Journal of Biological Chemistry 261: 10544-10550.

Rejman, J. and Kozubek, A. (2003). Inhibitory effect of natural phenolic lipids upon NADdependent dehydrogenases and on triglyceride accumulation in 3T3-L1 cells in culture. Journal of Agricultural and Food Chemistry 52: 246-250.

Rhew, T. H. and Sachan, D. S. (1983). Effects of carnitine and its precursors on alcohol-induced fatty liver. Federation Proceedings 42: 1308-1308 (Abstract 5960).

Rhew, T. H. and Sachan, D. S. (1984). Dose dependent effect of carnitine on ethanol-induced hepatic steatosis. Federation Proceedings 43: 395-395 (abstract 645). 
Rhew, T. H. and Sachan, D. S. (1986). Dose-dependent lipotropic effect of carnitine in chronicalcoholic rats. Journal of Nutrition 116: 2263-2269.

Ricketts, M.-L., Moore, D. D., Banz, W. J., Mezei, O. and Shay, N. F. (2005). Molecular mechanisms of action of the soy isoflavones includes activation of promiscuous nuclear receptors. A review. Journal of Nutritional Biochemistry 16: 321-330.

Riggs, T. R. and Hegsted, D. M. (1948). The effect of pantothenic acid deficiency on acetylation in rats. Journal of Biological Chemistry 172: 539-545.

Rikans, L. L., Arata, D. and Cederquist, D. C. (1965). Fatty livers produced in albino rats by excess niacin in high fat diets. II. Effect of choline supplements. Journal of Nutrition 85: 107-112.

Rong, N., Ausman, L. and Nicolosi, R. (1997). Oryzanol decreases cholesterol absorption and aortic fatty streaks in hamsters. Lipids 32: 303-309.

Roongpisuthipong, C., Kantawan, R. and Roongpisuthipong, W. (2007). Reduction of adipose tissue and body weight: effect of water soluble calcium hydroxycitrate in Garcinia atroviridis on the short term treatment of obese women in Thailand. Asia Pacific Journal of Clinical Nutrition 16: $25-29$.

Rosenfeld, B. (1973). Regulation by dietary choline of hepatic fatty acid synthetase in the rat. Journal of Lipid Research 14: 557-562.

Ross, A. B., Chen, Y., Frank, J., Swanson, J. E., Parker, R. S., Kozubek, A., Lundh, T., Vessby, B., Aman, P. and Kamal-Eldin, A. (2004). Cereal alkylresorcinols elevate gamma-tocopherol levels in rats and inhibit $\gamma$-tocopherol metabolism in vitro. Journal of Nutrition 134: 506-510.

Rotenberg, S. and Eggum, B. O. (1986). The effect of purified pectins with and without saponins in the diet on selected lipid parameters in liver and blood-plasma of rats. Acta Agriculturae Scandinavica 36: 211-216.

Roy, S., Freake, H. C. and Fernandez, M. L. (2000). Dietary soluble fiber lowers plasma LDL cholesterol (LDL-C) by modulating hepatic cholesterol 7-alpha hydroxylase. FASEB Journal 14: A298 
Russakoff, A. H. and Blumberg, H. (1944). Choline as an adjuvant to the dietary therapy of cirrhosis of the liver. Annals of Internal Medicine 21: 848-862.

Sachan, D. S. and Rhew, T. H. (1982). Lipotropic effect of carnitine on alcohol-induced hepatic steatosis. American Journal of Clinical Nutrition 35: R32, page xx.

Sachan, D. S. and Rhew, T. H. (1983). Lipotropic effect of carnitine on alcohol-induced hepatic steatosis. Nutrition Reports International 27: 1221-1226.

Sachan, D. S., Rhew, T. H. and Ruark, R. A. (1984). Ameliorating effects of carnitine and its precursors on alcohol-induced fatty liver. American Journal of Clinical Nutrition 39: 738-744.

Saheb, J. L. and Demers, J. M. (1972). Effect of lipotropic factors on cholesterol metabolism in duckling. Annales de Biologie Animale Biochimie Biophysique 12: 149.

Sakakibara, K., Shibata, Y., Higashi, T., Sanada, S. and Shoji, J. (1975). Effect of ginseng saponins on cholesterol-metabolism. 1. Level and synthesis of serum and liver cholesterol in rats treated with ginsenosides. Chemical and Pharmaceutical Bulletin 23: 1009-1016.

Salama, R. H. M., Nassar, A. Y. A., Nafady, A. A. M. and Mohamed, H. H. T. (2007). A novel therapeutic drug (copper nicotinic acid complex) for non-alcoholic fatty liver. Liver International 27: 454-464.

Schade, H. and Saltman, P. (1959). Influence of nicotinic acid on hepatic cholesterol synthesis in rabbits. Proceedings of the Society for Experimental Biology and Medicine 102: 265-267.

Schaefer, A. E., McKibbin, J. M. and Elvehjem, C. A. (1942). Pantothenic acid deficiency studies in dogs. Journal of Biological Chemistry 143: 321-330.

Schneeman, B. O. and Richter, D. (1993). Changes in plasma and hepatic lipids, small-intestinal histology and pancreatic-enzyme activity due to aging and dietary fiber in rats. Journal of Nutrition 123: 1328-1337.

Schön, H. (1958). Effect of nicotinic acid on the cholesterol contents of rat-livers. Nature 182: 534. Schriewer, H., Krämer, U., Rutkowski, G. and Borgis, K.-J. (1979). Influence of silibin dihemisuccinate on fatty acid synthesis in rat liver. Arzneimittelforschung 29: 524-526. 
Schwarz, J. M., Chen, T. W. and Linfoot, P. (1999). Effect of hydroxycitrate on hepatic de novo lipogenesis, gluconeogenesis and glucose production in obese hyperinsulinemic subjects. Circulation 100 (Suppl. I): A1015 (pages 1195-1196).

Seetharamaiah, G. S. and Chandrasekhara, N. (1988). Hypocholesterolemic activity of oryzanol in rats. Nutrition Reports International 38: 927-935.

Seetharamaiah, G. S. and Chandrasekhara, N. (1993). Comparative hypocholesterolemic activities of oryzanol, curcumin and ferulic acid in rats. Journal of Food Science and Technology-Mysore 30: $249-252$.

Sekiya, M., Yahagi, N., Matsuzaka, T., Najima, Y., Nakakuki, M., Nagai, R., Ishibashi, S., Osuga, J.-i., Yamada, N. and Shimano, H. (2003). Polyunsaturated fatty acids ameliorate hepatic steatosis in obese mice by SREBP-1 suppression. Hepatology 38: 1529-1539.

Sener, G., Balkan, J., Cevikbas, U., Keyer-Uysal, M. and Uysal, M. (2004). Melatonin reduces cholesterol accumulation and prooxidant state induced by high cholesterol diet in the plasma, the liver and probably in the aorta of C57BL/6J mice. Journal of Pineal Research 36: 212-216.

Sharma, R. and Rukmini, C. (1986). Rice bran oil and hypocholesterolemia in rats. Lipids 21: 715717.

Shefer, S., Salen, G., Bullock, J., Nguyen, L. B., Ness, G. C., Vhao, Z. H., Belamarich, P. F., Chowdhary, I., Lerner, S., Batta, A. K. and Tint, G. S. (1994). The effect of increased hepatic sitosterol on the regulation of 3-hydroxy-3-methylglutaryl-CoA reductase and cholesterol 7-alphahydroxylase in the rat and sitosterolemic homozygotes. Hepatology 20: 213-219.

Shieh, J., Wu, H., Cheng, K. and Cheng, J. (2009). Melatonin ameliorates high fat diet-induced diabetes and stimulates glycogen synthesis via a PKC $\zeta$-Akt-GSK3 $\beta$ pathway in hepatic cells. Journal of Pineal Research 47: 339-344.

Shils, M. E. and Stewart, W. B. (1954). Development of portal fatty liver in rats on corn diets response to lipotropic agents. Proceedings of the Society for Experimental Biology and Medicine 85: 298-303. 
Shimoda, H., Tanaka, J., Kikuchi, M., Fukuda, T., Ito, H., Hatano, T. and Yoshidao, T. (2009).

Effect of polyphenol-rich extract from walnut on diet-induced hypertriglyceridemia in mice via enhancement of fatty acid oxidation in the liver. Journal of Agricultural and Food Chemistry 57: 1786-1792.

Shimura, S. and Hasegawa, T. (1993). Changes of lipid concentrations in liver and serum by administration of carnitine added diets in rats. Journal of Veterinary Medical Science 55: 845847.

Shin, J., Kim, Y. J., Choi, M. S., Woo, D. H. and Park, T. (2004). Phytosterols and lecithin do not have an additive effect in lowering plasma and hepatic cholesterol levels in diet-induced hypercholesterolemic rats. Biofactors 22: 173-175.

Shrestha, S., Ehlers, S. J., Lee, J.-Y., Fernandez, M.-L. and Koo, S. I. (2009). Dietary green tea extract lowers plasma and hepatic triglycerides and decreases the expression of sterol regulatory element-binding protein-1c mRNA and its responsive genes in fructose-fed, ovariectomized rats. Journal of Nutrition 139: 640-645.

Singal, S. A. and Eckstein, H. C. (1941). The lipotropic action of some sulfur-containing amino acids and related substances. Journal of Biological Chemistry 140: 27-34.

Singh, U., Yokota, K., Gupta, C. and Shinozuka, H. (1990). Choline deficiency activates phospholipases A2 and C in rat liver without affecting the activity of protein kinase C. Journal of Nutritional Biochemistry 1: 434-439.

Sirato-Yasumoto, S., Katsuta, M., Okuyama, Y., Takahashi, Y. and Ide, T. (2001). Effect of sesame seeds rich in sesamin and sesamolin on fatty acid oxidation in rat liver. Journal of Agricultural and Food Chemistry 49: 2647-2651.

Siripurkpong, P. and Na-Bangehang, K. (2009). Effects of niacin and chromium on the expression of ATP-binding cassette transporter A1 and apolipoprotein A-1 genes in HepG2 cells. Journal of Nutritional Biochemistry 20: 261-268. 
Song, B. J., Moon, K. H., Olsson, N. U. and Salem, N. (2008). Prevention of alcoholic fatty liver and mitochondrial dysfunction in the rat by long-chain poly unsaturated fatty acids. Journal of Hepatology 49: 262-273.

Soo-Jung, L., Min-Jung, K., Jung-Hye, S., Jeong-Gyun, K., Shin-Kwon, K. and Nak-Ju, S. (2009). The effect of garlic and medicinal plants extracts on the liver function and lipid metabolism of rats administered with alcohol. Journal of the Korean Society of Food Science and Nutrition 38: 561-568.

Spadaro, L., Magliocco, O., Spampinato, D., Piro, S., Oliveri, C., Alagona, C., Papa, G., Rabuazzo, A. M. and Purrello, F. (2008). Effects of n-3 polyunsaturated fatty acids in subjects with nonalcoholic fatty liver disease. Digestive and Liver Disease 40: 194-199.

Spaniol, M., Kaufmann, P., Beier, K., Wuthrich, J., Torok, M., Scharnagl, H., Marz, W. and Krahenbuhl, S. (2003). Mechanisms of liver steatosis in rats with systemic carnitine deficiency due to treatment with trimethylhydraziniumpropionate. Journal of Lipid Research 44: 144-153. Stewart, J. R., Fryer, E. B. and Fryer, H. C. (1987). Effects of dietary fiber, carbohydrate, lipid and protein-levels on serum and liver lipids in rats. Journal of Nutrition 117: 650-659.

Story, J. A., Baldino, A., Czarnecki, S. K. and Kritchevsky, D. (1981). Modification of liver cholesterol accumulation by dietary fiber in rats. Nutrition Reports International 24: 1213-1219.

Subramanian, P., Mirunalini, S., Pandi-Perumal, S. R., Trakht, I. and Cardinali, D. P. (2007). Melatonin treatment improves the antioxidant status and decreases lipid content in brain and liver of rats. European Journal of Pharmacology 571: 116-119.

Sugano, M., Ikeda, I., Imaizumi, K., Watanabe, M. and Andoh, M. (1982). Effects of $\beta$-sitosterol on the concentrations of serum and liver cholesterol and serum apolipoproteins in rats fed butter fat. Journal of Nutritional Science and Vitaminology 28: 117-126.

Sugatani, J., Wada, T., Osabe, M., Yamakawa, K., Yoshinari, K. and Miwa, M. (2006). Dietary inulin alleviates hepatic steatosis and xenobiotics-induced liver injury in rats fed a high-fat and 
high-sucrose diet: Association with the suppression of hepatic cytochrome P450 and hepatocyte nuclear factor 4 alpha expression. Drug Metabolism and Disposition 34: 1677-1687.

Suh, M. H., Yoo, S. H., Chang, P. S. and Lee, H. G. (2005). Antioxidative activity of microencapsulated $\gamma$-oryzanol on high cholesterol-fed rats. Journal of Agricultural and Food Chemistry 53: 9747-9750.

Sullivan, A., Triscari, J., Hamilton, J. and Miller, O. (1974a). Effect of (-)-hydroxycitrate upon the accumulation of lipid in the rat: II. Appetite. Lipids 9: 129-134.

Sullivan, A., Triscari, J., Hamilton, J., Miller, O. and Wheatley, V. (1974b). Effect of (-)hydroxycitrate upon the accumulation of lipid in the rat: I. Lipogenesis. Lipids 9: 121-128.

Sullivan, A., Triscari, J. and Miller, O. (1974c). The influence of (-)-hydroxycitrate on in vivo rates of hepatic glycogenesis, lipogenesis and cholesterogenesis. Federation Proceedings 33: 656. Sullivan, A., Triscari, J. and Spiegel, J. (1977). Metabolic regulation as a control for lipid disorders. II. Influence of (-)-hydroxycitrate on genetically and experimentally induced hypertriglyceridemia in the rat. American Journal of Clinical Nutrition 30: 777-784.

Sullivan, A. C., Hamilton, J. G., Miller, O. N. and Wheatley, V. R. (1972). Inhibition of lipogenesis in rat liver by (-)-hydroxycitrate. Archives of Biochemistry and Biophysics 150: 183-190.

Sun, G. Y., Xia, J. M., Xu, J. F., Allenbrand, B., Simonyi, A., Rudeen, P. K. and Sun, A. Y. (1999). Dietary supplementation of grape polyphenols to rats ameliorates chronic ethanol-induced changes in hepatic morphology without altering changes in hepatic lipids. Journal of Nutrition 129: $1814-1819$.

Sung, J. H., Choi, S. J., Lee, S. W., Park, K. H. and Moon, T. W. (2004). Isoflavones found in korean soybean paste as 3-hydroxy-3-methylglutaryl coenzyme A reductase inhibitors. Bioscience, Biotechnology, and Biochemistry 68: 1051-1058.

Takeuchi, H., Nakamoto, T., Mori, Y., Kawakami, M., Mabuchi, H., Ohishi, Y., Ichikawa, N., Koike, A. and Masuda, K. (2001). Comparative effects of dietary fat types on hepatic enzyme 
activities related to the synthesis and oxidation of fatty acid and to lipogenesis in rats. Bioscience, Biotechnology, and Biochemistry 65: 1748-1754.

Tao, R. C., Peck, G. K. and Yoshimura, N. N. (1981). Effect of carnitine on liver fat and nitrogenbalance in intravenously fed growing-rats. Journal of Nutrition 111: 171-177.

Theriault, A., Casaschi, A. and Ota, D. (2002). Secretion of hepatic triglyceride-rich lipoprotein is inhibited by the flavonoid, taxifolin, via reduced DGAT and MTP activity. Arteriosclerosis Thrombosis and Vascular Biology 22: P423.

Thomas, M., Leelamma, S. and Kurup, P. A. (1983). Effect of blackgram fiber (Phaseolus mungo) on hepatic hydroxymethylglutaryl-CoA eeductase-activity, cholesterogenesis and cholesterol degradation in Rats. Journal of Nutrition 113: 1104-1108.

Thurston, J. H., Carroll, J. E., Hauhart, R. E. and Schiro, J. A. (1985). A single therapeutic dose of valproate affects liver carbohydrate, fat, adenylate, amino-acid, coenzyme A, and carnitine metabolism in infant mice: possible clinical-significance. Life Sciences 36: 1643-1651.

Thurston, J. H. and Hauhart, R. E. (1992). Amelioration of adverse effects of valproic acid on ketogenesis and liver coenzyme A metabolism by cotreatment with pantothenate and carnitine in developing mice: possible clinical significance. Pediatric Research 31: 419-423.

Tinker, L. F., Davis, P. A. and Schneeman, B. O. (1994). Prune fiber or pectin compared with cellulose lowers plasma and liver lipids in rats with diet-induced hyperlipidemia. Journal of Nutrition 124: 31-40.

Tojo, H., Igura, T., Nonomura, S., Sumi, R., Bando, Y. and Ito, T. (2008). A beneficial effect of polyphenol oligomers, oligonol, on choline deficiency-induced fatty liver in mice. Chemistry and Physics of Lipids 154: S15-S15.

Tokmakjian, S. and Haines, D. S. M. (1979). Influence of dietary choline intake upon liver ethanolamine metabolism. Canadian Journal of Biochemistry 57: 566-572. 
Topping, D. L., Oakenfull, D., Trimble, R. P. and Illman, R. J. (1988). A viscous fiber (methylcellulose) lowers blood-glucose and plasma triacylglycerols and increases liver-glycogen independently of volatile fatty-acid production in the rat. British Journal of Nutrition 59: 21-30. Toussant, M. J., Wilson, M. D. and Clarke, S. D. (1981). Coordinate suppression of liver acetylCoA carboxylase and fatty acid synthetase by polyunsaturated fat. Journal of Nutrition 111: 146153.

Tsai, C. E. and Tsai, Y. (1999). Effect of dietary fiber on the prevention of liver lipid accumulation induced by high polyunsaturated oil. Journal of Food Lipids 6: 75-89.

Tsuduki, T., Nakamura, Y., Honma, T., Nakagawa, K., Kimura, T., Ikeda, I. and Miyazawa, T. (2009). Intake of 1-deoxynojirimycin suppresses lipid accumulation through activation of the $\beta$ oxidation system in rat liver. Journal of Agricultural and Food Chemistry 57: 11024-11029.

Tsuruoka, N., Kidokoro, A., Matsumoto, I., Abe, K. and Kiso, Y. (2005). Modulating effect of sesamin, a functional lignan in sesame seeds, on the transcription levels of lipid- and alcoholmetabolizing enzymes in rat liver: A DNA microarray study. Bioscience, Biotechnology and Biochemistry 69: 179-188.

Tucker, H. F. and Eckstein, H. C. (1937). The effect of supplementary methionine and cystine on the production of fatty livers by diet. Journal of Biological Chemistry 121: 479-484.

Tucker, H. F. and Eckstein, H. C. (1938). The effect of supplementary lysine, methionine, and cystine on the production of fatty livers by high fat diets containing gliadin. Journal of Biological Chemistry 126: 117-123.

Turchetto, E., Infante, R. and Sechi, A. M. (1955). Effect of thyroxin on lipotropic activity of pantothenic acid. Bollettino- Societa Italiana Biologia Sperimentale (Napoli) 31: 233-234.

Tyner, E. P., Lewis, H. B. and Eckstein, H. C. (1950). Niacin and the ability of cystine to augment deposition of liver fat. Journal of Biological Chemistry 187: 651-654. 
Uchida, K., Mizuno, H., Hirota, K., Takeda, K., Takeuchi, N. and Ishikawa, Y. (1983). Effects of spinasterol and sitosterol on plasma and liver cholesterol levels and biliary and fecal sterol and bile-acid excretions in mice. Japanese Journal of Pharmacology 33: 103-112.

Ueshima, T., Shigata, Y., Wada, M., Oji, K. and Yoshida, T. (1956). Studies on the metabolism of pantothenic acid in liver damage. I. Urinary excretion of pantothenic acid in patients with various liver diseases and its correlation with liver function. Journal of Vitaminology 2: 299-306.

Vaishwanar, I., Jiddewar, G. G., Shukla, R. D. and Kowale, C. N. (1972). Effect of nicotinic-acid on serum and hepatic lipids in experimentally induced fatty liver. Indian Journal of Experimenta Biology 10: 428-430.

van der Hoorn, J. W. A., de Haan, W., Berbee, J. F. P., Havekes, L. M., Jukema, J. W., Rensen, P. C. N. and Princen, H. M. G. (2008). Niacin increases HDL by reducing hepatic expression and plasma levels of cholesteryl ester transfer protein in APOE*3Leiden.CETP mice. Arteriosclerosis Thrombosis and Vascular Biology 28: 2016-2022.

Wang, J. Q., Li, J., Zou, Y. H., Cheng, W. M., Lu, C., Zhang, L., Ge, J. F., Huang, C., Jin, Y., Lv, X. W., Hu, C. M. and Liu, L. P. (2009a). Preventive effects of total flavonoids of Litsea coreana leve on hepatic steatosis in rats fed with high fat diet. Journal of Ethnopharmacology 121: 54-60.

Wang, M., Liu, J.-R., Gao, J.-M., Parry, J. W. and Wei, Y.-M. (2009b). Antioxidant activity of tartary buckwheat bran extract and its effect on the lipid profile of hyperlipidemic rats. Journal of Agricultural and Food Chemistry 57: 5106-5112

Wang, W., Basinger, A., Neese, R. A., Shane, B., Myong, S. A., Christiansen, M. and Hellerstein, M. K. (2001). Effect of nicotinic acid administration on hepatic very low density lipoproteintriglyceride production. American Journal of Physiology-Endocrinology and Metabolism $2 \mathbf{2 8 0}$ E540-E547.

Wang, X., Song, K.-S., Guo, Q.-X. and Tian, W.-X. (2003). The galloyl moiety of green tea catechins is the critical structural feature to inhibit fatty-acid synthase. Biochemical Pharmacology 66: 2039-2047. 
Wang, X. and Tian, W. (2001). Green tea epigallocatechin gallate: a natural inhibitor of fatty-acid synthase. Biochemical and Biophysical Research Communications 288: 1200-1206.

Watson, J. A., Fang, M. and Lowenstein, J. M. (1969). Tricarballylate and hydroxycitrate: substrate and inhibitor of ATP:citrate oxaloacetate lyase. Archives of Biochemistry and Biophysics 135: 209-217.

Whitehead, C. C., McNab, J. M. and Griffin, H. D. (1981). The effects of low dietary concentrations of saponin on liver lipid-accumulation and performance in laying hens. British Poultry Science 22: 281-288.

Wijendran, V., Pronczuk, A., Beer, M. and Hayes, K. C. (2002). Free phytosterols lower plasma and liver lipids and increases fecal cholesterol excretion comparable to esterified sterols and stanols. FASEB Journal 16: A242-A242.

Wilcox, L. J., Borradaile, N. M., de Dreu, L. E. and Huff, M. W. (2001). Secretion of hepatocyte apoB is inhibited by the flavonoids, naringenin and hesperetin, via reduced activity and expression of ACAT2 and MTP. Journal of Lipid Research 42: 725-734.

Williams, M. A., Chu, L. C., McIntosh, D. J. and Hincenbe.I (1968). Effects of dietary fat level on pantothenate depletion and liver fatty acid composition in rat. Journal of Nutrition 94: 377-382.

Willumsen, N., Vaagenes, H., Rustan, A. C., Grav, H., Lundquist, M., Skattebol, L., Songstad, J. and Berge, R. K. (1997). Enhanced hepatic fatty acid oxidation and upregulated carnitine palmitoyltransferase II gene expression by methyl 3-thiaoctadeca-6,9,12,15-tetraenoate in rats. Journal of Lipid Mediators and Cell Signalling 17: 115-134.

Wirtschafter, Z. T. and Walsh, J. R. (1962). Hepatocellular lipoid changes in pantothenic acid deficiency. American Journal of Clinical Nutrition 10: 525-530.

Wojcicki, J., Samochowiec, L., Kadlubowska, D. and Lutomski, J. (1977). Studies on saponin fraction from root of Aralia mandshurica Rupr. et Maxim. Part IV. Influence of saponosides on content of lipids in blood serum and liver in experimental hyperlipemia. Herba Polonica 23: 285289 
Wright, R. S., Anderson, J. W. and Bridges, S. R. (1990). Propionate inhibits hepatocyte lipidsynthesis. Proceedings of the Society for Experimental Biology and Medicine 195: 26-29.

Wu, Z. M., Zhang, Y., Qi, Y., Lu, J. H., Tan, N. Z., Yin, W. T. and Zhu, J. S. (2005). Geraniol, policosanol, and SB formula: discrepant inhibitory expressions of liver cholesterol synthesis and serum lipids. FASEB Journal 19: A972-A972.

Yacowitz, H., Fleischman, A. I. and Kritchevsky, D. (1976). Does fiber in bread affect serum and liver lipids in rats. Journal of Nutrition 106: R26-R26.

Yagi, K. and Kotaki, A. (1969). The effect of massive doses of myo-inositol on hepatic phospholipid metabolism. Annals of the New York Academy of Sciences 165: 710-725.

Yang, D.-J., Chang, Y.-Y., Hsu, C.-L., Liu, C.-W., Lin, Y.-L., Lin, Y.-H., Liu, K.-C. and Chen, Y.C. (2010a). Antiobesity and hypolipidemic effects of polyphenol-rich longan (Dimocarpus longans Lour.) flower water extract in hypercaloric-dietary rats. Journal of Agricultural and Food Chemistry 58: 2020-2027.

Yang, M.-Y., Peng, C.-H., Chan, K.-C., Yang, Y.-S., Huang, C.-N. and Wang, C.-J. (2010b). The hypolipidemic effect of Hibiscus sabdariffa polyphenols via inhibiting lipogenesis and promoting hepatic lipid clearance. Journal of Agricultural and Food Chemistry 58: 850-859.

Yang, S., Tseng, J., Chang, Y. and Chen, Y. (2009). Flaxseed oil attenuates nonalcoholic fatty liver of hyperlipidemic hamsters. Journal of Agricultural and Food Chemistry 57.

Yeh, Y. Y. (1979). The opposing effects of nicotinic-acid and dibutyryl cyclic adenosine-3',5'monophosphate on ketogenesis in isolated rat hepatocytes. Journal of Nutrition 109: 110-118.

Yeh, Y.-Y. and Liu, L. (2001). Cholesterol-lowering effect of garlic extracts and organosulfur compounds: human and animal studies. Journal of Nutrition 131: 989S-993.

Yeh, Y.-Y. and Yeh, S.-M. (1994). Garlic reduces plasma lipids by inhibiting hepatic cholesterol and triacylglycerol synthesis. Lipids 29: 189-193. 


\section{Page 263 of 267}

\section{Critical Reviews in Food Science and Nutrition}

Yokota, F., Esashi, T., Takahash.S and Suzue, R. (1974). Effects of excess methionine and glycine on incorporation of sodium acetate-1 $1-^{14} \mathrm{C}$ into lipid of rat liver. Nutrition Reports International 10: 405-408.

Young, R. J., Lucas, C. C., Patterson, J. M. and Best, C. H. (1965). Lipotropic dose-response studies in rats: comparisons of choline, betaine, and methionine. Canadian Journal of Biochemistry and Physiology 34: 713.

Youssef, J., Cunningham, M. L. and Badr, M. (1994). Down-regulation of hepatic peroxisomal beta-oxidation due to pantothenic acid-deficiency. FASEB Journal 8: A736-A736.

Zeisel, S., Da Costa, K., Franklin, P., Alexander, E., Lamont, J., Sheard, N. and Beiser, A. (1991) Choline, an essential nutrient for humans. FASEB J 5: 2093-2098.

Zhang, L.-H., Kamanna, V. S., Zhang, M. C. and Kashyap, M. L. (2008). Niacin inhibits surface expression of ATP synthase $\beta$ chain in HepG2 cells: implications for raising HDL. Journal of Lipid Research 49: 1195-1201.

Zhang, R., Xiao, W., Wang, X., Wu, X. and Tian, W. (2006). Novel inhibitors of fatty-acid synthase from green tea (Camellia sinensis Xihu Longjing) with high activity and a new reacting site. Biotechnology and Applied Biochemistry 43: 1-7. 


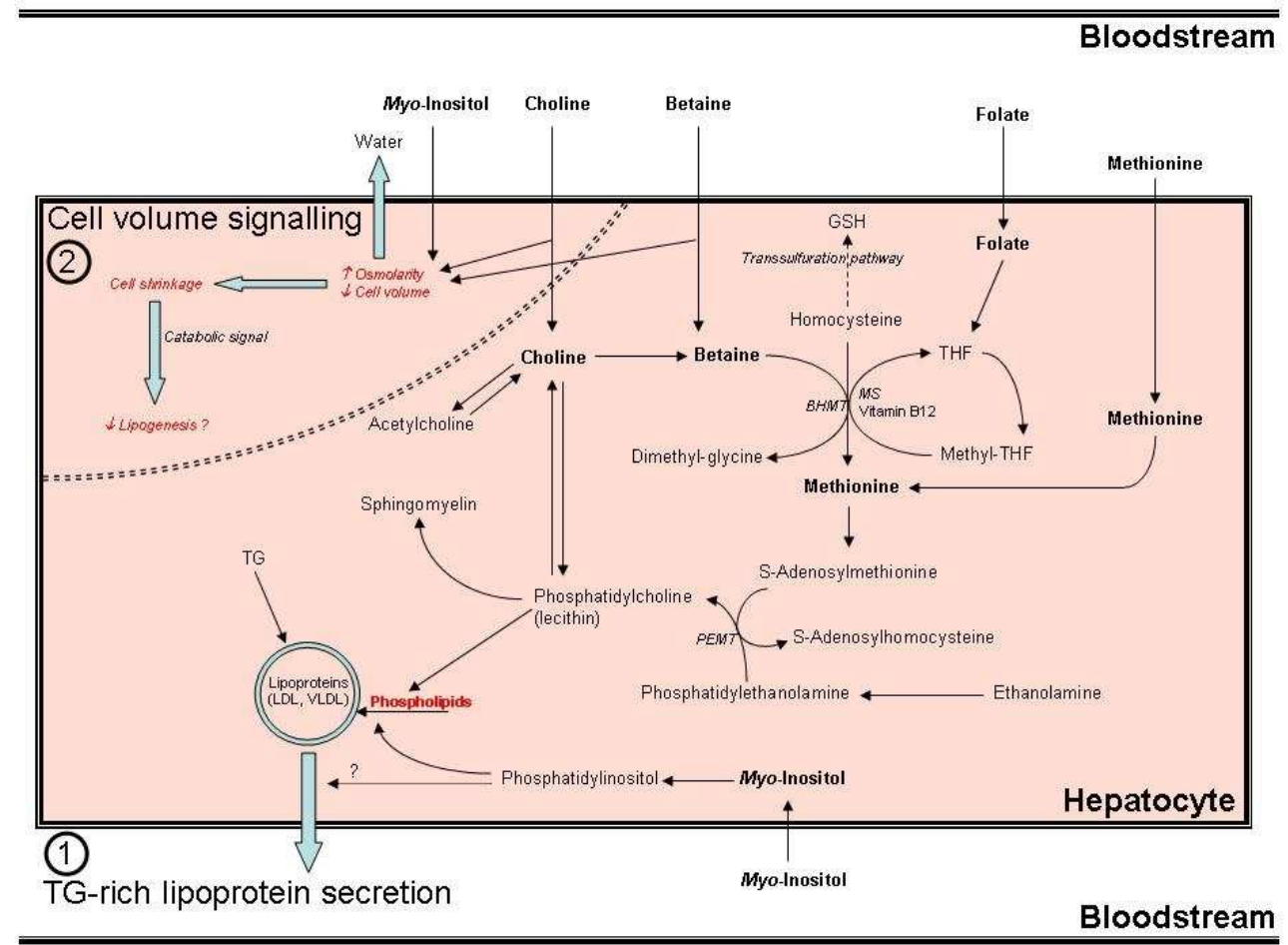

Figure 2A

$254 \times 190 \mathrm{~mm}(96 \times 96$ DPI) 


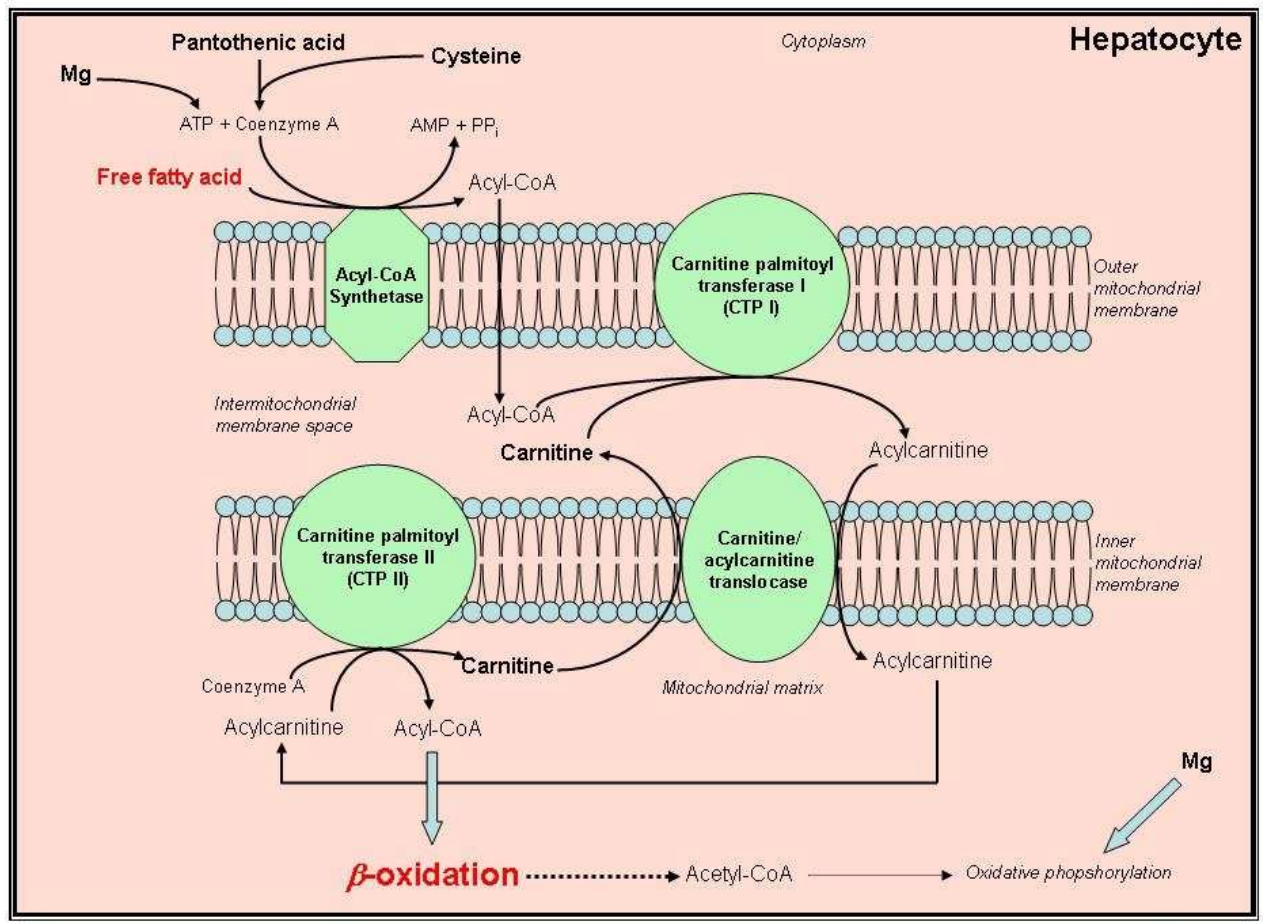

Figure $2 \mathrm{~B}$

$254 \times 190 \mathrm{~mm}(96 \times 96$ DPI) 


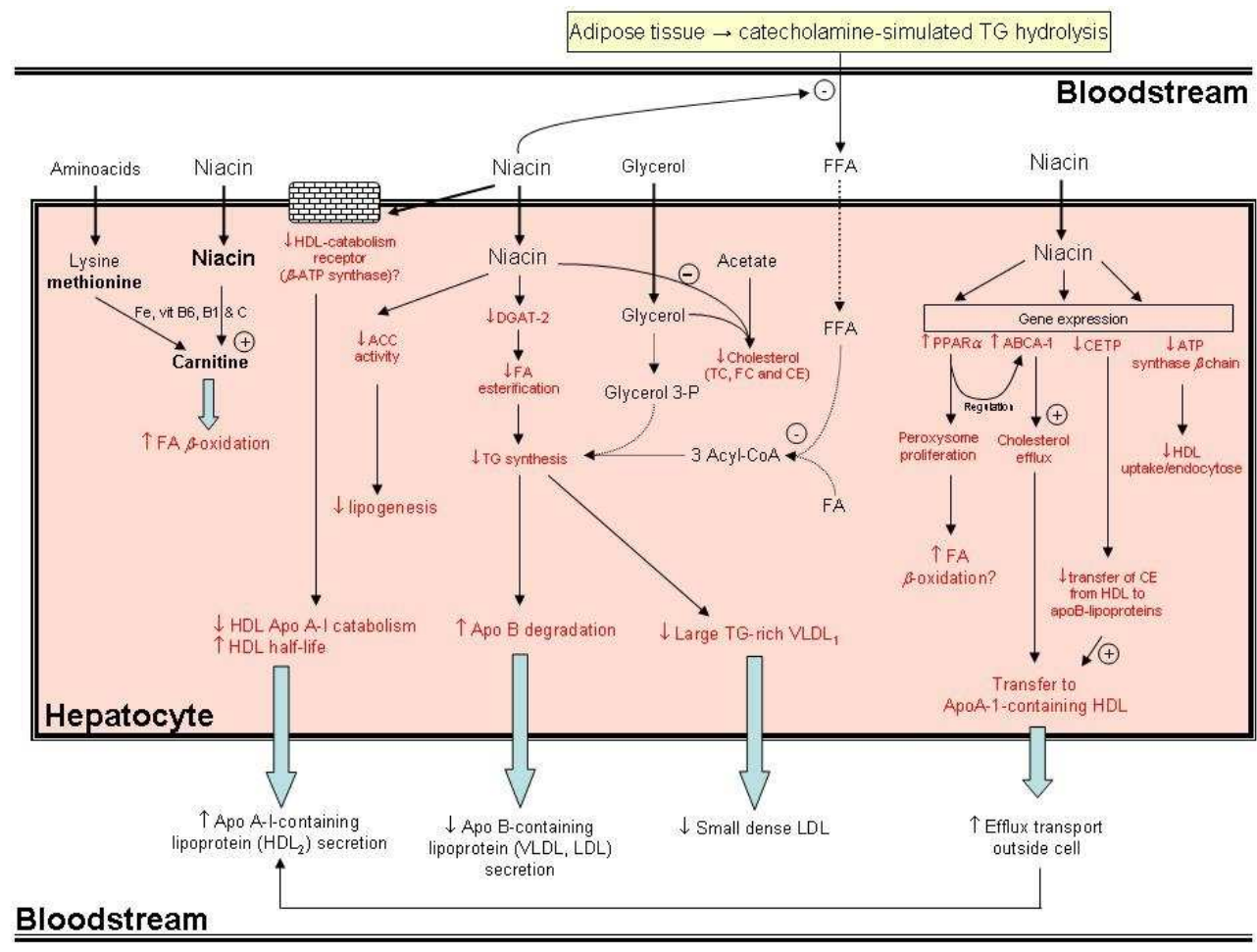

Figure $2 \mathrm{C}$

$254 \times 190 \mathrm{~mm}(96 \times 96$ DPI $)$

URL: http://mc.manuscriptcentral.com/bfsn Email: fergc@foodsci.umass.edu 


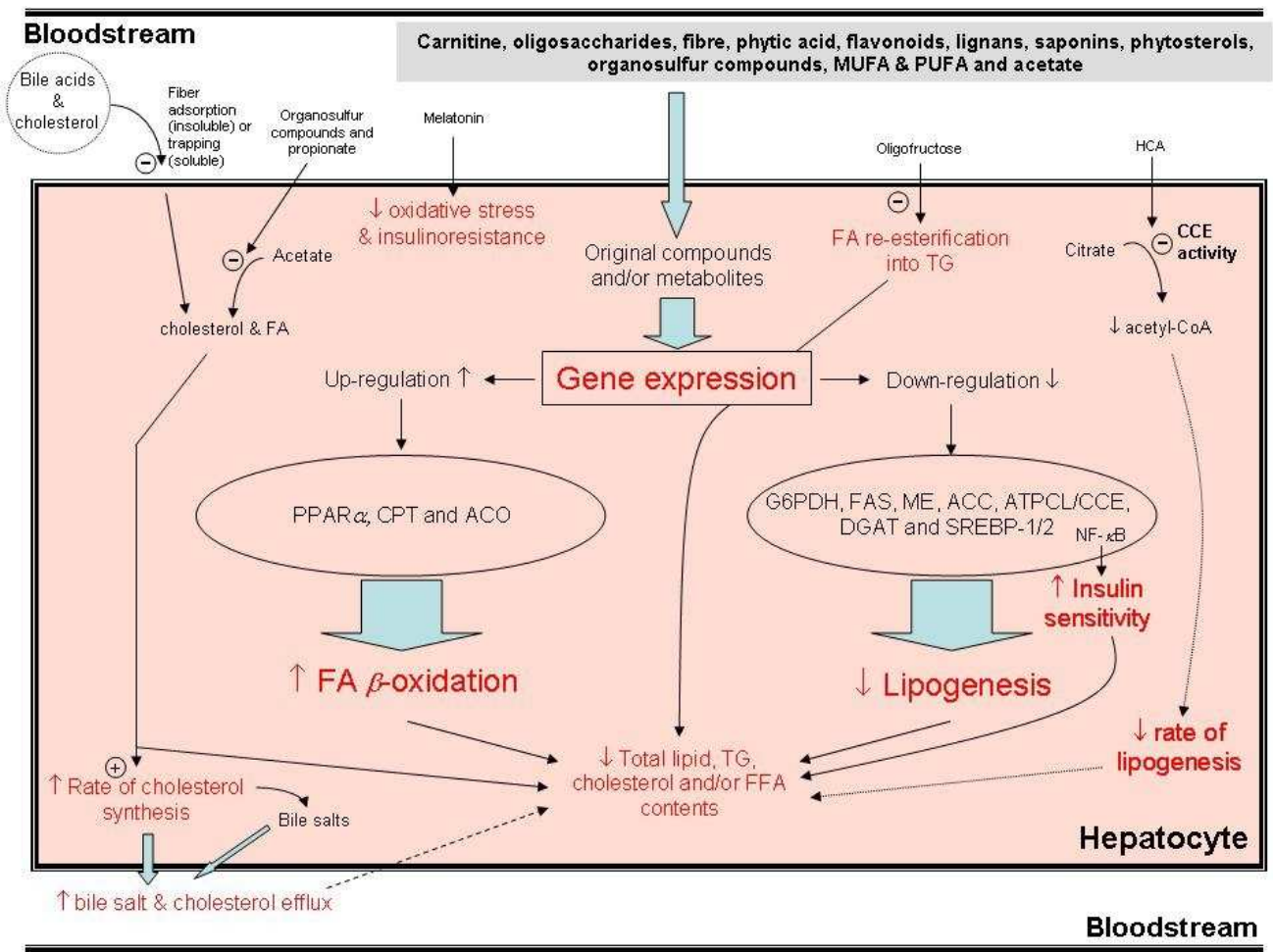

Figure $2 \mathrm{D}$

$254 \times 190 \mathrm{~mm}(96 \times 96 \mathrm{DPI})$

URL: http://mc.manuscriptcentral.com/bfsn Email: fergc@foodsci.umass.edu 